\title{
Agonist-induced modulation of glycocalyx barrier properties in the microcirculation : role in blood volume recruitment and oxygen exchange in the heart
}

Citation for published version (APA):

Brands, J. (2010). Agonist-induced modulation of glycocalyx barrier properties in the microcirculation : role in blood volume recruitment and oxygen exchange in the heart. [Doctoral Thesis, Maastricht University]. Maastricht University. https://doi.org/10.26481/dis.20101029jb

Document status and date:

Published: 01/01/2010

DOI:

10.26481/dis.20101029jb

Document Version:

Publisher's PDF, also known as Version of record

Please check the document version of this publication:

- A submitted manuscript is the version of the article upon submission and before peer-review. There can be important differences between the submitted version and the official published version of record.

People interested in the research are advised to contact the author for the final version of the publication, or visit the DOI to the publisher's website.

- The final author version and the galley proof are versions of the publication after peer review.

- The final published version features the final layout of the paper including the volume, issue and page numbers.

Link to publication

\footnotetext{
General rights rights.

- You may freely distribute the URL identifying the publication in the public portal. please follow below link for the End User Agreement:

www.umlib.nl/taverne-license

Take down policy

If you believe that this document breaches copyright please contact us at:

repository@maastrichtuniversity.nl

providing details and we will investigate your claim.
}

Copyright and moral rights for the publications made accessible in the public portal are retained by the authors and/or other copyright owners and it is a condition of accessing publications that users recognise and abide by the legal requirements associated with these

- Users may download and print one copy of any publication from the public portal for the purpose of private study or research.

- You may not further distribute the material or use it for any profit-making activity or commercial gain

If the publication is distributed under the terms of Article 25fa of the Dutch Copyright Act, indicated by the "Taverne" license above, 
Agonist-induced modulation of glycocalyx barrier properties in the microcirculation

Role in blood volume recruitment and oxygen exchange in the heart 
(C) Judith Brands, Maastricht 2010

Agonist-induced modulation of glycocalyx barrier properties in the microcirculation: Role in blood volume recruitment and oxygen exchange in the heart; PhD thesis Maastricht University

Cover: Electron microscopic visualization of glycocalyx structures in a capillary by Bernard van den Berg

Printed by Ponsen \& Looijen

ISBN: 978-90-6464-410-8 


\section{Agonist-induced modulation of glycocalyx barrier properties in the microcirculation}

Role in blood volume recruitment and oxygen exchange in the heart

\section{PROEFSCHRIFT}

ter verkrijging van de graad van doctor aan de Universiteit Maastricht, op gezag van de Rector Magnificus,

Prof. mr. G.P.M.F. Mols volgens het besluit van het College van Decanen

in het openbaar te verdedigen

op vrijdag 29 oktober 2010 om 12.00 uur

door

Judith Brands

geboren te Oss 


\section{Promotores:}

Prof. dr. H. Vink (Universiteit van Amsterdam \& Universiteit Maastricht)

Prof. dr. ir. J.A.E. Spaan (Universiteit van Amsterdam)

\section{Copromotor:}

Dr. J.W.G.E. Van Teeffelen

\section{Beoordelingscommissie:}

Prof. dr. ir. M.G.J. Arts

Prof. dr. J.B. Bassingthwaighte (University of Washington)

Prof. dr. D.J.G.M. Duncker (Erasmus Universiteit Rotterdam)

Prof. dr. F.W. Prinzen

Prof. dr. F.S. Villanueva (University of Pittsburgh)

Prof. dr. J.L. Waltenberger

(Part of) The research described in this thesis was supported by a grant of the Netherlands Heart Foundation (NHF-2003B181). Financial support by the Netherlands Heart Foundation for the publication of this thesis is gratefully acknowledged. 


\section{Contents}

1 Introduction

2 Role for glycocalyx perturbation in atherosclerosis development and associated microvascular dysfunction

3 Bradykinin- and sodium nitroprusside-induced increases in capillary tube hematocrit in mouse cremaster muscle are associated with impaired glycocalyx barrier properties

4 Acute hyaluronidase treatment reduced systemic glycocalyx volume in mice

5 Whole-body recruitment of glycocalyx volume during intravenous adenosine infusion in goats

6 Comparison of four mathematical models for analysis of intravascular indicator dilution curves in the coronary circulation

7 Acute attenuation of glycocalyx barrier properties increases coronary blood volume independently of coronary flow reserve

8 Hyaluronidase-induced recruitment of glycocalyx volume increases myocardial oxygen exchange in the goat heart

9 Discussion 
References

Summary

Samenvatting

183

Dankwoord

195

Curriculum Vitae

199 


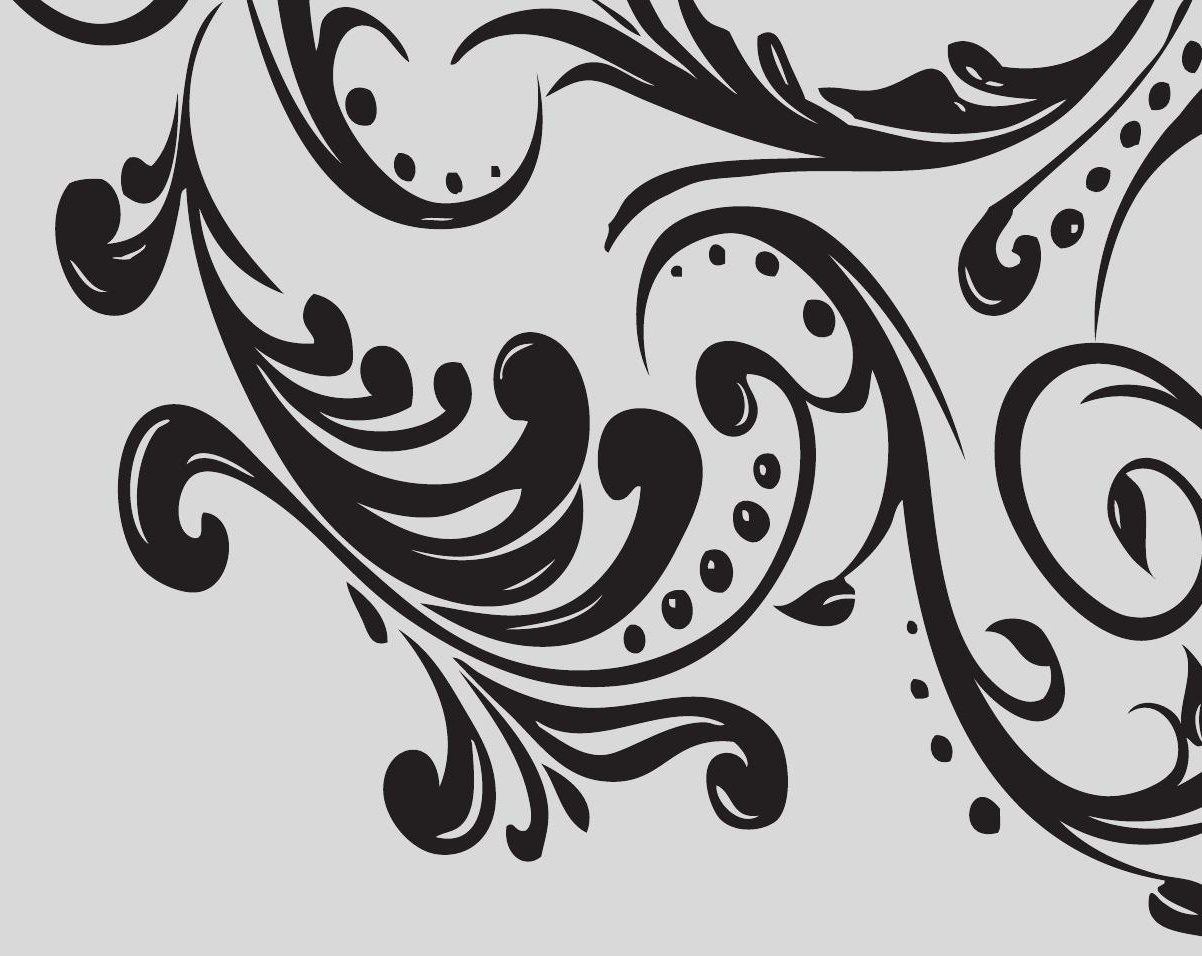

\section{Introduction}






\section{Background}

The heart, see figure 1.1 and 1.2, is a hollow muscular organ that pumps blood through the vascular system. The heart muscle itself is supplied with oxygen and nutrients by the right and left coronary arteries, which arise from the root of the aorta just above the aortic valve cusps and run over the surface of the heart, the epicardium. The epicardial arteries branch into arteries that penetrate the muscle and branch further into arterioles. Healthy arterial vessels are essential for adequate regulation of coronary blood flow to match the supply of oxygen to myocardial oxygen demand. However, every year there are a considerable number of patients with symptoms of chest pain during exercise who do not have a prominent stenosis in their coronary arteries or coronary artery disease at all; patients with cardiac syndrome X [62]. In addition, the stenosis detected in a large part of the patients with a lesion is hemodynamically non-significant [109]. In all these cases, the angina may relate to coronary microvascular dysfunction [13].



Figure 1.1 Base of the heart (adapted from [98]). 
Downstream of the arterial vessels, exchange of oxygen takes place in a branching network of very thin and small blood vessels, the capillary bed. This network is interwoven with the muscle fibers such that the diffusion distance between the capillaries and the myocardial cells is small. The function of the capillaries is to exchange oxygen, nutrients, and other substances between the blood and the interstitial fluid. The wall of these tiny blood vessels consist of only one cell layer, the endothelium, which acts as a barrier between the distinct compartments of the internal medium, the blood and the interstitial space and myocytes. On the luminal side of the endothelial cells a hydrated layer, the glycocalyx, is situated. The glycocalyx is structurally composed of proteoglycans, glycosaminoglycans, glycoproteins, glycolipids and adsorbed plasma proteins. Although coronary blood flow is regulated in small arteries and arterioles with diameters ranging between 400 and $10 \mu \mathrm{m}$, the capillaries might contribute to the regulation of oxygen exchange by modulating the glycocalyx and thereby increasing microvascular blood volume and surface area for exchange.

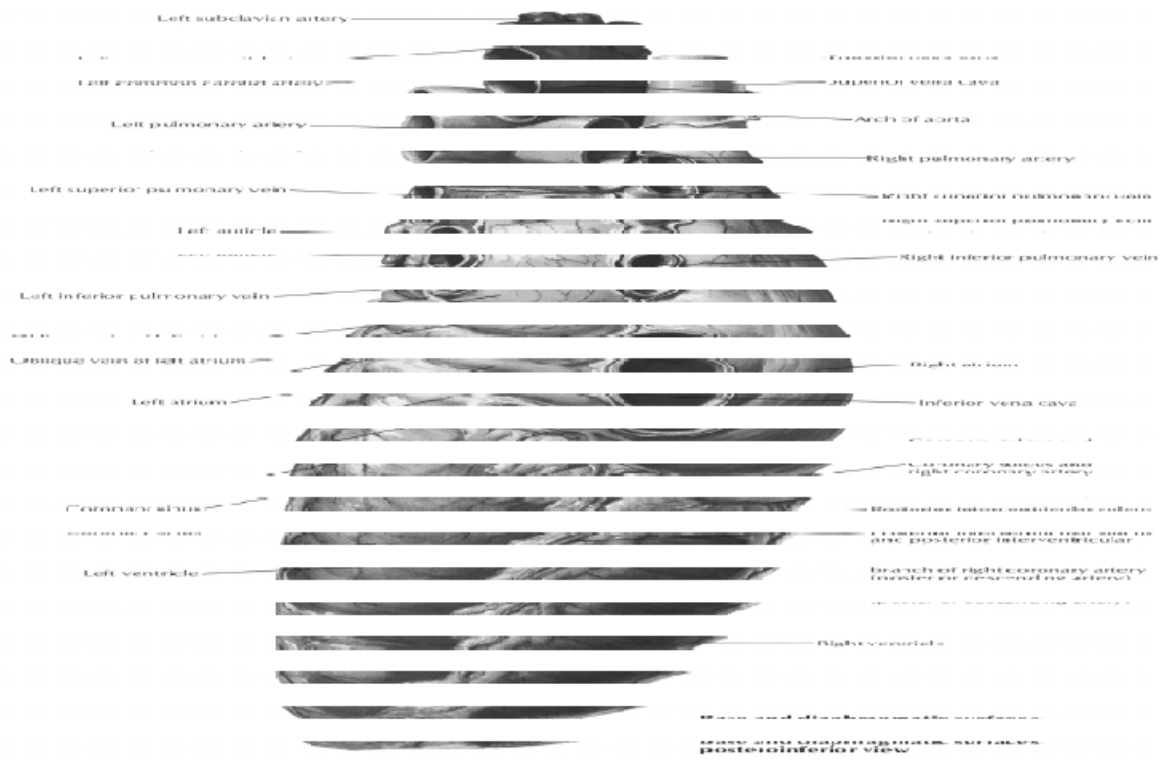

Figure 1.2 Base and diaphragmatic surfaces (adapted from [98]). 
In the microvasculature a substantial part of the anatomic vascular volume is occupied by endothelial glycocalyx $[47,100,103,110,111,121,152,157,158]$, see figure 1.3 . The endothelial glycocalyx is a blood-excluding layer on the luminal side of endothelial cells throughout the entire vascular bed which occupies in the microvasculature a relative large fraction of available anatomic vascular volume. Its presence reduces blood perfused vascular volume, such as reflected by a reduction in capillary tube hematocrit $[20,29,157,158]$. Experiments on the cremaster muscle demonstrated that



Figure 1.3 Electron microscopic overview of Alcian blue 8GX-stained goat coronary capillary. Scale bar length is $1 \mu \mathrm{m}$.

capillary tube hematocrit increased during adenosine infusion $[29,74,110]$. It has been suggested that this was a result of an adenosine-induced reduction in exclusion properties of the glycocalyx. Evidence for the latter was provided by direct visualization of an increased macromolecular accessibility of the glycocalyx during superfusion of the 
cremaster with adenosine [110]. In the heart it has been demonstrated that adenosineinduced coronary hyperemia was insensitive to enzymatic degradation of the glycocalyx, in contrast to reactive hyperemia. This observation indicates that adenosine

might increase coronary conductance in the heart by impairing blood exclusion by the glycocalyx [154]. Effects of adenosine on actual circulating blood volume, and the contribution of a modulation of glycocalyx exclusion properties to this volume, were, however, not studied so far. In addition to the possible role of microvascular glycocalyx recruitment in the regulation of oxygen exchange by capillaries, degradation of the glycocalyx was found to be associated with an impaired shear-dependent NO-production, increased endothelial adhesion of leukocytes and platelets, increased leakage of water and proteins from the vasculature, and a reduction in number of perfused capillaries $[10,151]$. The microvascular problems associated with glycocalyx degradation might hinder an adequate exchange of oxygen to the myocardium [13]. Therefore, the absence of an intact glycocalyx might attenuate the oxygen exchange in more than one way.

\section{Aim of the study}

The studies presented in this thesis were performed to investigate the role of agonistinduced glycocalyx volume modulation in regulation of microvascular blood volume and oxygen exchange, primarily in the heart (figure 1.4). Our hypothesis is that modulation of the access of circulating blood to microvascular glycocalyx contributes to the regulation of coronary oxygen exchange. Thus, pharmacological vasodilators and endogenously produced substances may increase coronary blood volume not only by dilating the coronary resistance vessels but, in addition, by increasing functionally perfused microvascular volume. We further hypothesize that the increase in functionally perfused capillary volume by increased blood accessibility of the glycocalyx is associated with an increase in exchange capacity for oxygen and nutrients due to increased microvascular blood volume, larger surface area for exchange and/or increased microvascular permeability, thereby increasing the extraction of these substances during passage through the coronary system. 


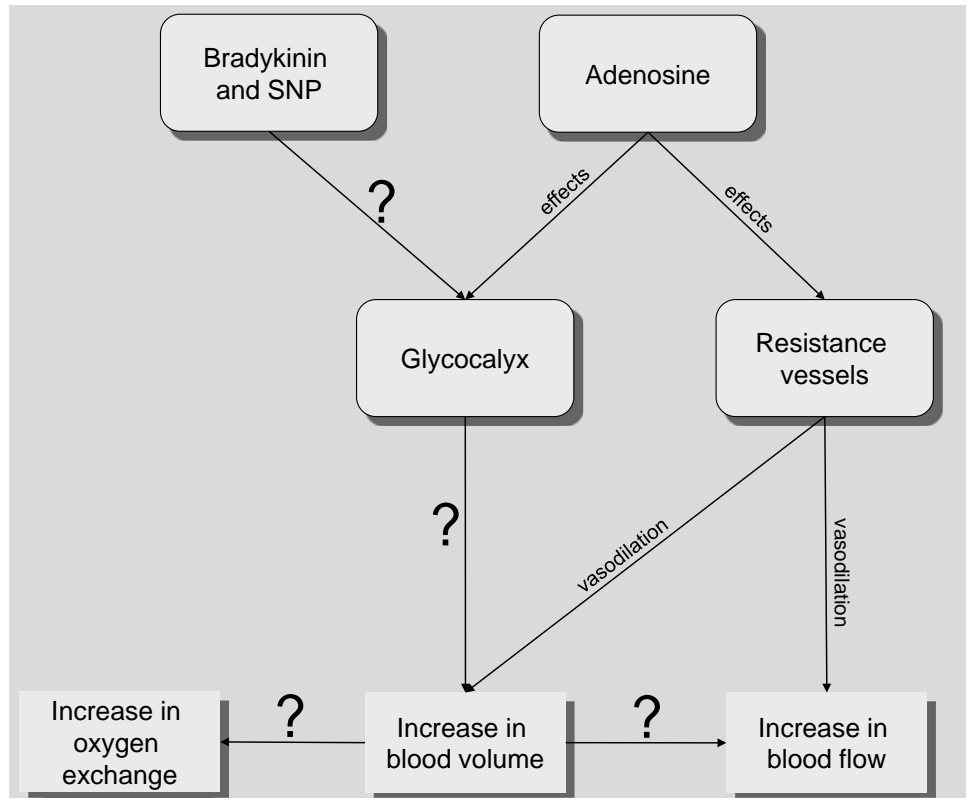

Figure 1.4 In the current thesis we will study agonist-induced glycocalyx modulation in capillaries, adenosine induced changes in glycocalyx volume and blood volume at whole body level and in the coronary circulation, and the effect of glycocalyx degradation on coronary oxygen exchange.

\section{Outline of the study}

Introduction Chapter 1 to 3 provides an introduction to the endothelial glycocalyx. First, the structure and function of the glycocalyx, and its role in vascular homeostasis will be discussed (chapter 2). In chapter 3 the concept of agonist-induced glycocalyx modulation as a mechanism to regulate perfused capillary volume was verified for the vasodilators bradykinin and sodium nitroprusside.

Systemic studies In chapter 4 and 5 systemic volume measurements of perfused and blood inaccessible glycocalyx volumes were performed to enable the study of agonist-induced glycocalyx modulation at a whole-body level. By comparing the dilution of a glycocalyx accessible and inaccessible indicator after their injection in the systemic circulation, the total anatomic vascular volume and blood accessible volume can be derived, respectively. The difference between the two volumes is defined as 
glycocalyx volume $[103,104]$. The systemic volume measurement technique was first validated in mice (chapter 4) after which it was applied in goats to measure the effect of adenosine infusion on whole-body glycocalyx volume (chapter 5).

Coronary studies Chapters 6-8 focus on blood volume measurements in the heart using the indicator dilution technique, and coronary oxygen exchange. First, the indicator dilution method will be introduced and four different models to analyze the coronary indicator dilution data compared (chapter 6). Subsequently, coronary blood volume measurements were performed in hearts of goats at baseline and during infusion of adenosine, and this was compared for control conditions, and conditions in which the glycocalyx was enzymatically provoked (chapter 7 ). As the final chapter of this part of the thesis, the effect of degradation of the coronary glycocalyx on coronary oxygen exchange in goat hearts was studied (chapter 8).

Discussion The last chapter (chapter 9) will discuss the findings of this thesis and give directions for further research. 



\section{Role for glycocalyx perturbation in atherosclerosis development} and associated microvascular dysfunction

The contents of this chapter are based on Judith Brands, Jurgen WGE Van Teeffelen, Bernard M Van den Berg and Hans Vink, Role for glycocalyx perturbation in atherosclerosis development and associated microvascular dysfunction (2007), Future Lipidology 


\section{Introduction}

Cardiovascular disease is the leading cause of death and illness in developed countries and atherosclerosis makes up the single most important contributor $[43,78]$. When focusing on the heart, the presence of a lesion can easily be overlooked as long as dilation of the vasculature distal from the lesion, i.e. the microcirculation, is able to compensate for the increase in resistance, and coronary blood flow can be maintained. However, when compensation by the microcirculation fails, coronary perfusion will become inadequate and consequently myocardial infarction might occur. The endothelial glycocalyx has been indicated to play a role in protection of the vascular wall against atherosclerotic insults as well as regulation of microvascular function. Both aspects are discussed in this review.

\section{Endothelial glycocalyx}

\section{Structure}

The endothelium is actively involved in a variety of vascular functions, such as regulation of vascular tone, fluid and solute exchange, hemostasis and coagulation, and inflammatory responses. At the luminal side of the endothelial cells a hydrated layer, the glycocalyx, is situated. The glycocalyx is structurally composed of proteoglycans, glycosaminoglycans, glycoproteins and glycolipids. Proteoglycans consist of a core protein with one or more linked glycosaminoglycan chains [93,114,117]. Most, i.e. 50-90\%, of the proteoglycans associated with endothelial cells are heparan sulfate proteoglycans (HSPG) [114]. HSPG are able to bind proteins with a heparin binding domain, such as factors involved in vascular growth, lipid metabolism, oxygen radical scavenging, and coagulation [95]. The HSPG are classified into families according to their core protein structure. The major HSPG within the cardiovascular system are syndecan, glypican, and perlecan [119]. Cell surface HSPG are associated with the plasma membrane according to three modes: direct linkage of the core protein into the lipid bilayer (syndecan), linkage through a glycosylphosphatidylinositol (GPI) anchor covalently bound to the core protein (glypican), and specific or relatively nonspecific interactions between heparan sulfate chains and other molecules associated with the plasma membrane (perlecan) [6,119]. Linkage through a GPI anchor localizes HSPG 
Glycocalyx perturbation in atherosclerosis and microvascular dysfunction

to lipid rafts including caveolae that are rich in signaling molecules [107]. The second most common glycosaminoglycan is chondroitin sulfate and is typically present in a ratio of 1:4 with heparan sulfate. The nonsulfated linear glycosaminoglycan hyaluronan, the only glycosaminoglycan not covalently linked to a peptide core, with a molecular weight up to $1 \times 10^{7} \mathrm{Da}$, contributes significantly to the hydration state of the glycocalyx [3]. The transmembrane CD44 receptor, which is localized in caveolae, and bonds with chondroitin sulfate chains linked to core proteins anchors hyaluronan to the plasma membrane [107]. For more detailed information on the structural glycocalyx components the reader is referred to the following reviews $[114,117,136,162]$. The polysaccharide structures together with adsorbed plasma proteins result in a significant thick endothelial glycocalyx. Various techniques to visualize the length of the polysaccharide structures with electron microscopy, an example is shown in figure $2.1 \mathrm{a}$, resulted in dimensions ranging from $20 \mathrm{~nm}$ up to $0.5 \mu \mathrm{m}[117,142]$. Using intravital microscopy of striated muscle preparations, an in vivo glycocalyx thickness of 0.2 to $0.5 \mu \mathrm{m}$ was estimated in hamsters and mice cremaster muscle from the difference in column width of brightfield capillary diameter (endothelial cell boundaries) and column width of a glycocalyx-excluded plasma tracer, such as high molecular weight fluorescein isothiocyanate (FITC)-dextrans [48, 111, 121, 157]. Microparticle image velocimetry in mouse cremaster muscle venules confirmed a significant exclusion of flowing plasma by the glycocalyx in vivo and resulted in estimates of a mean effective thickness of 0.3 to $0.4 \mu \mathrm{m}$ [129]. Combining intravascular microscopy with a theoretical simulation of flow in microvascular networks by Pries et al. resulted in glycocalyx layer estimates of approximately $0.55 \mu \mathrm{m}$ [115]. The same group, now using a flow resistance model, calculated for vessels with a diameter of 10 to $40 \mu \mathrm{m}$ a layer thickness of $0.8-1 \mu \mathrm{m}$ [113]. Analogous to the intravital microscopy studies which utilize the selective exclusion properties of the glycocalyx to determine its thickness in rodent cremaster capillaries, glycocalyx volume in the systemic circulation of humans has recently been estimated from the difference in distribution volume between circulating plasma, derived from the distribution of labeled red blood cells, and glycocalyx accessible dextrans. In healthy males, systemic glycocalyx volumes of 1.5-1.7 liter were reported $[103,104]$. 


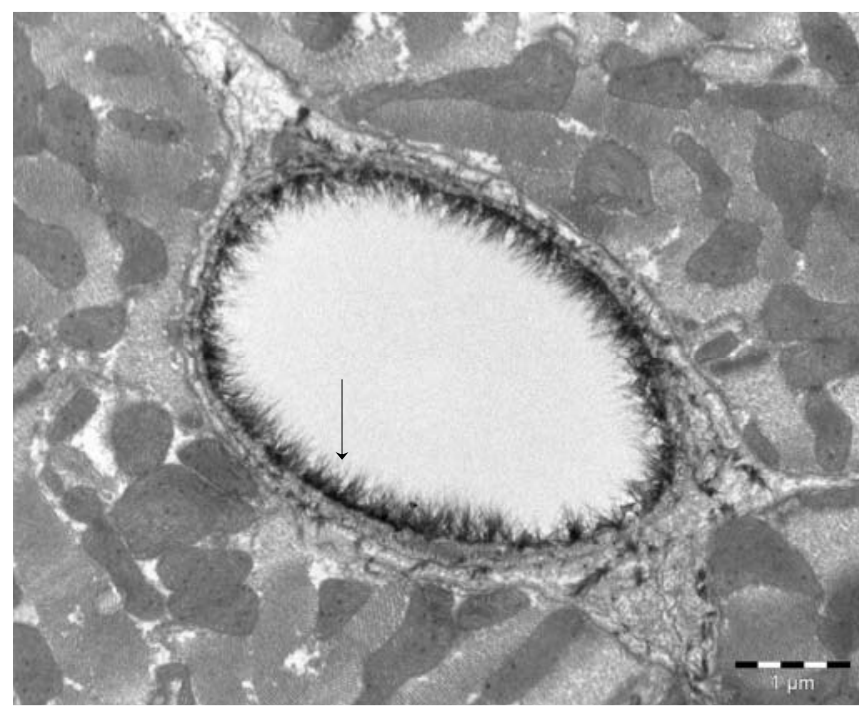

(a) Control

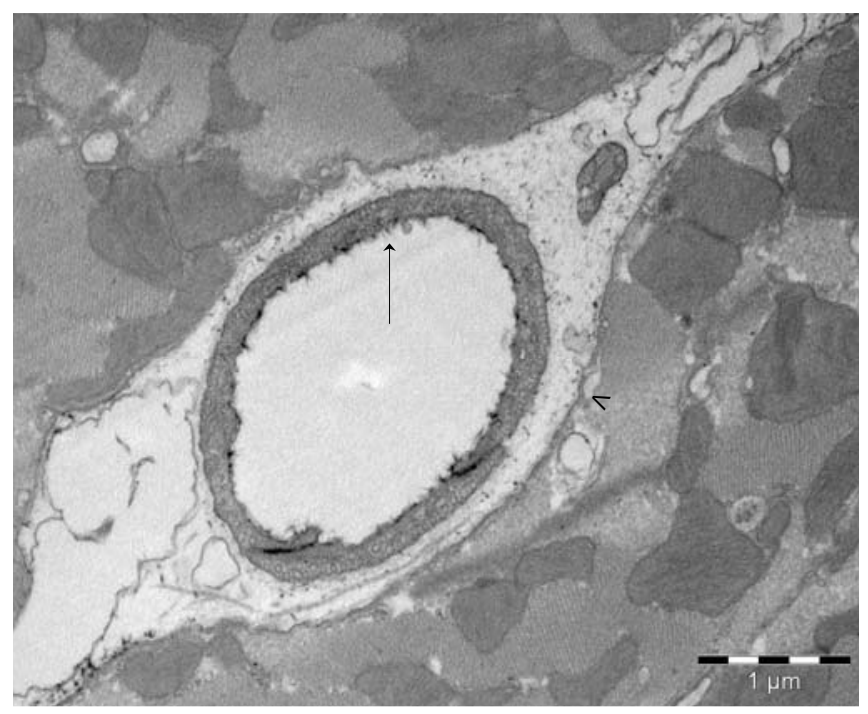

(b) Hyaluronidase

Figure 2.1 Electron microscopic visualization of glycocalyx structures in a capillary. (a) Electron microscopy overview of an Alcian blue 8GX-stained rat left ventricular myocardial capillary (bar $=1$ $\mu \mathrm{m})$. The glycocalyx is indicated by an arrow. (b) Enzymatic treatment resulted in a condensed endothelial surface staining, see arrow, and notable perivascular myocardial tissue edema indicated by the arrowhead (bar=1 $\mu \mathrm{m})$. 
Glycocalyx perturbation in atherosclerosis and microvascular dysfunction

\section{Function}

The last decade, experimental evidence has been presented indicating that the glycocalyx plays an important role in vascular homeostasis. Modification of glycocalyx structures in the vasculature by enzymes such as pronase, heparinase, and hyaluronidase were found to result in an increased leakage of fluid and solutes across the glycocalyx and the vessel wall $[11,52]$, edema formation [142] as shown in figure $2.1 \mathrm{~b}$, impairment in flow-induced endothelial NO production [90,104], and increased adhesion of leukocytes $[21,48]$. Many of the vascular changes evoked by enzymatic degradation of the glycocalyx also characterize conditions of atherosclerosis. The possible role of the glycocalyx in atherogenic protection is discussed in the following paragraph.

\section{Atherosclerosis}

\section{Reduced glycocalyx at atherosclerotic prone sites}

A role for glycocalyx perturbation in atherosclerosis was recently suggested by Van den Berg et al. [141]. They showed that dimensions of the endothelial glycocalyx at the sinus region of the mouse internal carotid artery, a region prone for the development of atherosclerosis, were significantly less than the glycocalyx dimensions found at the common carotid artery. This reduction in glycocalyx thickness was accompanied by a greater intima-to-media-ratio and a thicker subendothelial layer, which both are processes associated with the development of atherosclerosis. Together with the observation in cultured endothelial cells that, in comparison to static conditions, fluid shear stress stimulates incorporation of hyaluronan in the endothelial glycocalyx [38], it was hypothesized that the thinner glycocalyx at the sinus region was caused by disturbed flow patterns at this region. Thereupon, we hypothesize the presence of vascular regions in the circulation that exhibit an increased risk for developing atherosclerotic insults due to a less protective glycocalyx profile as a result from disturbances in local flow patterns. 


\section{Reduced glycocalyx upon exposure to atherogenic risk factors}

In addition to its susceptibility to disturbances in flow, it has been demonstrated that the glycocalyx is reduced in the presence of atherogenic risk factors. In comparison to control mice, an atherogenic mouse model was shown to exhibit a reduction in glycocalyx dimension in the common carotid artery and this reduction was accompanied by an increase in intima-to-media-ratio and intima thickness [141]. These findings are consistent with studies demonstrating substantial loss of glycocalyx during hyperglycemic conditions $[99,103,104]$, and upon acute exposure to atherogenic or inflammatory stimuli $[20,21,48]$.

\section{Hyperglycemic conditions}

Shedding of glycocalyx constituents in the circulation during high glucose was suggested upon observed increased circulating plasma levels of hyaluronan and reduced systemic glycocalyx volumes after hyperglycemic clamping of healthy volunteers. Infusion of the antioxidant $\mathrm{N}$-acetylcysteine prevented the glycocalyx reduction under these conditions thus implying that the generation of reactive oxygen species contributed to glycocalyx perturbation [104]. Furthermore, in patients with long-standing type 1 diabetes a reduced systemic glycocalyx volume was observed in comparison with normoglycemic control subjects [103]. A more pronounced reduction in systemic glycocalyx was seen in the type 1 patients with microalbuminuria compared with patients without microalbuminuria. Again, reduction in glycocalyx volume correlated with increased levels of circulating hyaluronan [99,103]. Since hyaluronan levels were found to correlate to intima-to-media thickness [99], these data altogether argue that disturbances of hyaluronan metabolism might be associated with a reduced glycocalyx, vascular damage and accelerated atherogenesis.

\section{Acute stimulation with ox-LDL and TNF- $\alpha$}

In hamster cremaster muscle, exclusion properties of the glycocalyx were impaired after the infusion of tumor-necrosis factor- $\alpha$ (TNF- $\alpha$ ), a pro-inflammatory cytokine. The effect of TNF- $\alpha$ was more extensive compared to degradation by hyaluronidase [48], suggesting that TNF- $\alpha$ probably not only removes hyaluronan from the glycocalyx but other components as well. Similarly, infusion of clinically relevant concen- 
Glycocalyx perturbation in atherosclerosis and microvascular dysfunction

trations of oxidized low density lipoprotein (ox-LDL), but not normal LDL, reduced glycocalyx thickness in mouse cremaster capillaries, which could be prevented by administration of superoxide dismutase and catalase [156], suggesting once more a role for oxygen-derived free radicals [20]. Capillary tube hematocrit increased simultaneously with the decrease in glycocalyx dimensions after the infusion of ox-LDL without causing significant changes in capillary red blood cell velocity, suggesting that ox-LDL increases volume accessible to red bloods cells by removal of proteoglycans and/or adsorbed proteins from the glycocalyx [20]. In a subsequent study, infusion of ox-LDL increased the number of adherent leukocytes to the endothelial surface and reduced leukocyte rolling velocity in a way that corresponded to treatment of the glycocalyx with the enzyme heparitinase [21]. Interestingly, infusion of heparan sulfate and heparin could prevent the ox-LDL induced leukocyte adhesion, and since it was shown that these components were bound to the endothelial surface the authors suggested that a damaged glycocalyx could rapidly be restored via administration of its structural components and thereby preventing adhesion of leukocytes to the endothelium.

In summary, anatomic location as well as various atherogenic risk factors are associated with perturbation of the glycocalyx on the one hand, while enzymatic degradation of its constituents is associated with impaired vascular protection on the other hand. We, therefore, hypothesize that perturbation of the glycocalyx by prevailing risk factors contributes to the process of lesion development and microvascular dysfunction in atherosclerosis.

\section{Blood flow regulation in the microvasculature}

Under normal physiological conditions, there is a close match between coronary blood flow and myocardial oxygen consumption rate. At rest the myocardium already extracts $60-70 \%$ of the oxygen delivered by coronary blood flow, limiting the oxygen extraction reserve. Upon continuous interruption of the oxygen supply the affected myocardium quickly stops beating, showing that adequate blood supply is essential for proper myocardial function $[138,139]$. Dilatory compensation by vessels distal from a stenosis is necessary for adequate blood supply when a lesion develops in the coronary vascular bed. Three different regulatory mechanisms have been proposed to 
be involved in the dilation of the resistance vessels, and each mechanism seems to dominate in different domains of the network. Studying isolated coronary microvessels it was found that large arteries $(80-150 \mu \mathrm{m})$ were more sensitive to flow, myogenic mechanisms predominated in vessels between $50-80 \mu \mathrm{m}$, while small arterioles $(<50 \mu \mathrm{m})$ showed the greatest sensitivity to metabolites [58]. In addition, a role for the capillaries in skeletal and cardiac muscle blood flow regulation has been suggested [68,123], but the importance of this compartment for the compensation and the mechanisms involved in the recruitment of capillary volume are not well understood at the moment. Nevertheless, the vascular changes associated with atherosclerosis have been shown to extend into the microcirculation, as evidenced by an impaired endothelial dependent vasodilatation, which may occur even before a significant lesion develops, and a reduction in number of capillaries $[76,133,153]$. Therefore, not only the atherosclerotic lesion itself but also the associated microvascular deficiency in compensatory blood flow regulation can ultimately contribute to the development of a myocardial infarction. The possible effects of a perturbed glycocalyx on blood flow regulation is considered in the following sections.

\section{Glycocalyx plays a role in NO mediated flow dependent dilation}

An important role for the glycocalyx in shear dependent NO production and associated resistance vessel dilation has been shown in experimental studies. Many of these studies have recently been reviewed by Tarbell and Pahakis [136]. Enzymatic degradation of hyaluronan [90] in isolated arteries resulted in an impaired NO response to fluid flow, while the importance of heparan sulfates in shear dependent NO release was shown in cultured endothelial cells [34]. Recently, the role of many of the different glycocalyx components in shear dependent NO production in cultured endothelial cells was compared in a single study. Glycoproteins bearing acidic oligosaccharides with terminal sialic acids, heparan sulfates, chondroitin sulfates and hyaluronic acids were considered by corresponding enzyme treatment with neuraminidase, heparinase, chondroitinase and hyaluronidase, while the NO dependent agonists bradykinin and histamine were utilized to check the viability of the endothelial cell NO production system [107]. The results showed that shear-induced NO production was significantly reduced after removal of heparan sulfates, sialic acids, and hyaluronan, but not after removal of chondroitin sulfates. The impairment of NO production was specific for 
Glycocalyx perturbation in atherosclerosis and microvascular dysfunction

the shear stimulus since histamine and bradykinin remained capable of inducing a significant increase in NO production after any of the enzyme treatments. Noteworthy, hyaluronic acid and heparan sulfate, unlike chondroitin sulfate, are both linked to caveolae that are rich in signaling molecules including enzymatic active nitric oxide synthase (eNOS) supporting the idea that NO mediated flow dependent dilation is a glypican-caveolae-eNOS mechanism $[107,118]$. The exact mechanism is still unknown but it has been suggested that with increasing flow heparan sulfate undergo a shear stress-dependent conformational change from a random coil to an unfurled filamenthelix structure. This produces additional anionic binding sites for sodium ions of the blood, which could carry on the signal transduction into the endothelial cell for a vasodilatory reaction [128]. More evidence indicating that heparan sulfates play a pivotal role in mechanotransduction of shear stress was recently provided by the study of Yao et al. [166]. These investigators showed that heparinase treated endothelial cells no longer align after 24 hours of laminar flow, and that they proliferate as if there were no flow present, showing that removal of the heparan sulfates appears to turn off the endothelial cell response to shear stress.

In addition to the polysaccharide structures, a role for adsorbed proteins with a heparin-binding domain within the glycocalyx was found with respect to its mechanotransduction properties in vivo [150]. Presumed release of these proteins from the glycocalyx by infusion of heparin resulted in a diminished NO mediated dilator response to shear stress during reactive hyperemia in mice cremaster muscle arterioles. This was accompanied by a significant leakage of large dextrans from the circulation, suggesting impairment in glycocalyx exclusion properties. Taken together, these data show that perturbation of the glycocalyx can contribute to an impaired NO mediated flow dependent dilation, which is generally regarded to be a hallmark of cardiovascular disease.

\section{Glycocalyx influences microvascular resistance}

Intravital microscopy studies in cremaster tissue indicate that in control conditions the glycocalyx comprises a region of $20-40 \%$ of anatomic capillary volume, which excludes blood components, such as flowing plasma, inert plasma macromolecules and red bloods cells, and consequently reduces functional capillary volume for blood flow and capillary tube hematocrit [157]. The exclusion properties of the glycocalyx 
seem, however, to be very dynamic and to be influenced by pathological stimuli as well as agonists. Superfusion of the cremaster muscle with adenosine, for example, was found to reduce the ability of the glycocalyx to exclude fluorescently labeled large size dextrans by more than $50 \%$ while at supraphysiological concentrations of adenosine even red blood cell exclusion was significantly reduced [110]. In line with this, adenosine-induced coronary hyperemia was, unlike coronary hyperemia after a transient ischemic stimulus, demonstrated not to be limited by the presence of the endothelial glycocalyx. Degradation of hyaluronan from the glycocalyx was found to solely increase coronary conductance during reactive hyperemia by $\sim 40 \%$ without affecting adenosine-induced coronary hyperemia, thereby abolishing the difference between reactive hyperemia and adenosine-induced hyperemia that was present during control conditions. These data suggest that adenosine can cause the 'recruitment' of glycocalyx volume by impairing its exclusion properties and this is associated with an increased functionally perfused volume particularly in the microvasculature. The $40 \%$ increase in coronary conductance during reactive hyperemia [154] corresponds nicely with the $14-21 \%$ decrease in flow resistance that was found in the rat mesenteric microcirculation after heparinase microinfusion [115]. Although these studies seem to suggest that glycocalyx loss might be beneficial for microvascular perfusion, degradation of the glycocalyx is also associated with an increase in platelet adhesion [115], edema formation [142], impaired flow-mediated dilation [90,104], and loss of capillary perfusion $[12,172]$, which ultimately will cause microvascular perfusion to be compromised.

\section{Conclusion}

The endothelial glycocalyx compartment resides on the luminal side of blood vessels. Data so far indicate that an intact glycocalyx contributes to the protection of endothelial function throughout the vasculature. Since a variety of atherogenic risk factors have been associated with endothelial glycocalyx perturbation, it is suggested that the glycocalyx might constitute a first line of defense against atherosclerotic insults. A thin glycocalyx at locations exposed to a disturbed blood flow therefore might facilitate lesion development in larger arteries. In the heart, adequate compensation of coronary perfusion via the microvasculature is needed to maintain coronary perfusion 




Figure 2.2 Perturbation of endothelial glycocalyx results in a pro-atherogenic state, as evidenced by activation of coagulation, platelet and leukocyte adhesion, impaired vascular barrier properties, and endothelial dysfunction.

in the presence of such atherogenic lesion; unfortunately, the compensation seems to be reduced upon glycocalyx degradation. At the arteriolar level, a damaged glycocalyx is associated with impaired shear stress dependent NO production, while at the capillary level, perturbation of the glycocalyx can result in reduction of functional capillary density and an inability to 'recruit' glycocalyx volume by agonists. In conclusion, the glycocalyx plays an important role in vascular homeostasis in both macroand microcirculation, this is summarized in figure 2.2 . 



\section{Bradykinin- and sodium} nitroprusside-induced increases in capillary tube hematocrit in mouse cremaster muscle are associated with impaired glycocalyx barrier properties

The contents of this chapter are based on Jurgen WGE Van Teeffelen, Alina A Constantinescu, Judith Brands, Jos AE Spaan, and Hans Vink, Bradykinin- and sodium nitroprusside-induced increases in capillary tube haematocrit in mouse cremaster muscle are associated with impaired glycocalyx barrier properties (2008), The Journal of Physiology 


\section{Abstract}

Previous studies have suggested that agonists may increase functionally perfused capillary volume by modulation of blood excluding glycocalyx volume, but direct evidence for this association is lacking at the moment. Using intravital microscopic visualization of mouse cremaster muscle, we determined the effects of bradykinin $\left(10^{-5} \mathrm{M}\right)$ and sodium nitroprusside $\left(10^{-6} \mathrm{M}\right)$ on capillary tube hematocrit and glycocalyx barrier properties. In control $\mathrm{C} 57 \mathrm{Bl} / 6$ mice $(\mathrm{N}=10)$, tube hematocrit in capillaries $(\mathrm{N}=71)$ increased $(\mathrm{P}<0.05)$ from $8.7 \pm 0.3 \%$ during baseline to $21.2 \pm 1.2 \%$ and $22.2 \pm 0.9 \%$ during superfusion with bradykinin and sodium nitroprusside respectively. In parallel, the exclusion zone of FITC-labeled $70 \mathrm{kDa}$ dextrans decreased $(\mathrm{P}<0.05)$ from $0.37 \pm 0.01 \mu \mathrm{m}$ during baseline to $0.17 \pm 0.01 \mu \mathrm{m}$ with bradykinin and $0.15 \pm 0.01$ $\mu \mathrm{m}$ with sodium nitroprusside. Bradykinin and sodium nitroprusside had no effect on dextran exclusion and tube hematocrit in capillaries $(\mathrm{N}=55)$ of hyperlipidemic ApoE3-Leiden mice, which showed impaired exclusion of $70 \mathrm{kDa}$ dextrans $(0.05 \pm$ $0.02 \mu \mathrm{m} ; \mathrm{P}<0.05$ vs $\mathrm{C} 57 \mathrm{Bl} / 6)$ and increased capillary tube hematocrit (23 $\pm 0.8 \%$; $\mathrm{P}<0.05$ vs $\mathrm{C} 57 \mathrm{Bl} / 6$ ) under baseline conditions, indicating impaired glycocalyx barrier properties. Our data show that vasodilator substances increase functionally perfused capillary volume and that this effect is associated with a reduction in glycocalyx exclusion of $70 \mathrm{kDa}$ dextrans. Modulation of glycocalyx volume might represent a novel mechanism of perfusion control at the capillary level.

\section{Introduction}

The endothelial glycocalyx forms a highly hydrated mesh of polysaccharide structures and adsorbed plasma proteins on the luminal side of the vessel wall [114,136]. Recent estimations suggest that the glycocalyx may occupy $\sim 1.5 \mathrm{~L}$ of the systemic vasculature in healthy subjects [104]. These estimations are based on the exclusion of circulating blood by the glycocalyx domain, as originally observed in intravital microscopy studies of muscle microcirculation in rodents [157]. Thus, under resting conditions the glycocalyx has been demonstrated to significantly exclude red blood cells (RBCs) and labeled macromolecules and to greatly retard flowing plasma [114,129, 157, 162]. These exclusion properties allow the glycocalyx to reduce functionally perfused cap- 
Agonist induced increase in hematocrit and impaired glycocalyx barrier properties

illary volume [157]. As a result, the glycocalyx has been demonstrated to contribute to the low tube hematocrit (i.e., $20-50 \%$ of systemic values) that is found in capillaries $[28,29,74,124]$ and to influence microvascular resistance [115, 154].

Intravital microscopy studies from Duling and coworkers in cremaster muscle originally showed that capillary tube hematocrit may increase during agonist and metabolic stimulation and they suggested the possibility that a reduction in glycocalyx exclusion was involved. Thus, tube hematocrit was found to be about fourfold higher during muscle activity and adenosine superfusion compared to the resting condition [74], while after treatment of the glycocalyx with heparinase, adenosine did not provoke a significant change in the already elevated capillary tube hematocrit anymore [29]. Using a simple two-compartment model with RBCs and plasma flowing in the central core of the capillary and a relatively slow moving plasma layer near the vessel wall representing the glycocalyx, the authors calculated that the increase in capillary tube hematocrit could be explained by a reduction in glycocalyx thickness of $1.2 \mu \mathrm{m}$ during contractions and adenosine [29,74]. Direct visualization of exclusion zones for fluorescently labeled large molecular weight dextrans and red blood cells in cremaster capillaries by the Duling laboratory and our own studies indicated glycocalyx dimensions that were smaller than $1.2 \mu \mathrm{m}$, i.e., in the range of about 0.2 to 0.7 $\mu \mathrm{m}[47,48,110,111,121,156-158]$. Nevertheless, Platts \& Duling demonstrated that during superfusion of the muscle with adenosine, exclusion of FITC-labeled $70 \mathrm{kDa}$ dextrans by the glycocalyx was rapidly and profoundly reduced [110]. This observation has prompted the hypothesis that adenosine can 'recruit' capillary volume for perfusion by modulating the accessibility of the glycocalyx for flowing blood [28, 151]. Unfortunately, concomitant measures of capillary hemodynamics were not made in the study of Platts \& Duling [110], and it is uncertain therefore how the increase in glycocalyx accessibility for $70 \mathrm{kDa}$ dextrans would influence capillary perfusion. In this respect it is worth mentioning that, in contrast to the profound change in dextran exclusion, RBC exclusion appeared modestly impaired (23\% reduction in exclusion zone) at pharmacological concentrations of adenosine only [110].

In the present study we therefore aimed at determining the potential of vasodilatorinduced modulation of glycocalyx accessibility to increase capillary perfusion. We evaluated the effects of an endothelium-dependent dilator, bradykinin, and an endothelium-independent dilator, sodium nitroprusside, on both capillary tube hema- 
tocrit and exclusion properties of the glycocalyx. In mouse cremaster muscle, exclusion of RBCs and fluorescently labeled $70 \mathrm{kDa}$ dextrans was determined together with measurements of RBC flux and velocity, and capillary anatomical diameter, under baseline conditions and in the presence of dilator in the superfusate. Since it has been proposed that the glycocalyx layer is affected by $\mathrm{RBC}$ velocity with a decrease in its thickness resulting from increases in velocity or shear rate $[74,115,124]$, we put particular focus on the relation between capillary tube hematocrit/glycocalyx exclusion and $\mathrm{RBC}$ velocity during baseline and vasodilator administration. Measurements were done in healthy $\mathrm{C} 57 \mathrm{Bl} / 6$ mice and in hyperlipidemic ApoE3-Leiden mice, used as experimental model of atherogenic degradation of the glycocalyx. Atherogenic degradation of the glycocalyx in cremaster capillaries was recently shown after intravenous bolus administration of oxidized lipoproteins [20].

\section{Methods}

\section{General surgery and anesthesia}

All procedures and protocols were approved by, and carried out according to the guidelines of the Animal Care and Use committee of the Academic Medical Center. Experiments were performed on male mice (25-30 g body wt). C57Bl/6 mice $(\mathrm{N}=10)$ were obtained from Charles River Europe. ApoE3-Leiden mice ( $\mathrm{N}=10)$, transgenic strain 2, were obtained from the Gaubius Laboratorium (TNO-PG Leiden, The Netherlands) and were cross-bred with $\mathrm{C} 57 \mathrm{Bl} / 6$ mice [147]; transgenic animals of the F10-F11 generation, identified by PCR analysis of genomic DNA from the ear pavilion, were used for the current experiments [153]. C57Bl/6 mice received standard rat/mouse chow (AM-2, Hope Farms; Woerden, The Netherlands), and ApoE3-Leiden mice were placed at the age of $8 \mathrm{wk}$ on a cholesterol-enriched high-fat diet $(0.5 \%$ cholate, $15 \%$ cocoa butter, $1 \%$ cholesterol, $40.5 \%$ sucrose, $10 \%$ corn starch, $1 \%$ corn oil, and $4.7 \%$ cellulose; HFC $0.5 \%$ diet, Hope Farms) for 3 months. This diet was shown to result in an increase in plasma cholesterol and triglyceride levels in this period of time [153]. At the beginning of an experiment, mice were anesthetized with an intraperitoneal (ip) injection of ketamine hydrochloride $(125 \mathrm{mg} / \mathrm{kg})$ and medetomidine $(0.2 \mathrm{mg} / \mathrm{kg})$, and tracheotomised to ensure airway patency [153]. Depth of anesthesia was maintained 
Agonist induced increase in hematocrit and impaired glycocalyx barrier properties

according to stability of blood pressure, respiration rate, and lack of toe pinch reflex by supplemental administration (every $\sim 1 \mathrm{~h}$ ) of anesthetic (ketamine: $15 \mathrm{mg} / \mathrm{kg}$, ip; medetomidine: $35 \mu \mathrm{g} / \mathrm{kg}$, ip). To counter effects of decreases in blood pressure and heart rate induced by medetomidine, atropine was administered at $1 \mathrm{~h}$ intervals during the experiment (initial dose: $0.5 \mathrm{mg} / \mathrm{kg}$, subcutaneous (sc); maintenance: 0.125 $\mathrm{mg} / \mathrm{kg}$, ip). The right carotid artery and jugular vein were canulated for monitoring systemic blood pressure and administration of fluorescent dextrans, respectively. Esophageal temperature was maintained at $\sim 37^{\circ} \mathrm{C}$ by radiant heat. No fluid was supplemented during the experiment except for the anesthesia and dextrans. At the end of the experimental procedures, a blood sample was collected in a heparinized capillary $(50 \mu \mathrm{l})$ using tail bleeding for determination of systemic hematocrit, followed by an overdose of ketamine.

\section{Mouse cremaster preparation and video microscopy}

The mouse was placed in a supine position on a custom built animal platform and the right cremaster muscle was prepared $[20,153]$. Briefly, an incision was made through the skin and the muscle dissected from the surrounding connective tissue. The exposed muscle was positioned on a clear Silicon pedestal and longitudinally incised from the apex to the inguinal canal with minimal disruption of the vascular supply. After severing the deferential artery and vein, the testis and epididymis were dissected away and repositioned in the abdominal cavity. The cremaster muscle was spread radially on the pedestal and pinned at the edges. The muscle was continuously $(\sim 5 \mathrm{ml} / \mathrm{min})$ superfused at $34^{\circ} \mathrm{C}$ with a bicarbonate-buffered physiological salt solution (PSS) of the following composition (in $\mathrm{mM}$ ): $131.9 \mathrm{NaCl}, 4.7 \mathrm{KCl}, 2.0 \mathrm{CaCl}_{2}, 1.2 \mathrm{MgSO}_{4}, 20$ $\mathrm{NaHCO}_{3}$ and equilibrated with $5 \% \mathrm{CO}_{2}-95 \% \mathrm{~N}_{2}$ to obtain a $\mathrm{pH}$ of $\sim 7.4$.

Following surgery, the preparation was transferred to the stage of an intravital microscope (Olympus BHM), coupled to a cooled intensified CCD video camera (GenIV ICCD, Princeton Instruments). Microvessels were observed using bright-field (100 W Hg lamp) microscopy (condensor: Olympus MA20, numerical aperture (NA): 0.4) or epi-illuminated (100 W Hg lamp) for the examination of fluorescent dextrans. Brightfield images were made with a $435 \mathrm{~nm}$ band pass interference filter (blue light) in the light path; fluorescent tracers were visualized using appropriate filters for FITC and Texas Red. Cremaster muscle capillaries were examined with a $\times 60$ water immersion 
objective lens (Olympus; LUMPlanFL, NA 0.9), and arterioles were examined with an $\times 20$ objective lens (Olympus; MSPlan 20, NA 0.4). Images were displayed on a Philips CM 8833-II video monitor and recorded using a SVHS video tape recorder (JVC BR-S611E) and a time coding interface unit (JVC SA-F911E) for post-hoc data analysis.

\section{Experimental protocol}

The preparation was equilibrated for $30 \mathrm{~min}$, during which time the arteriolar network was scanned for the presence of vasomotor tone. This evaluation of tone was based on the diameter range for these vessels established in a previous study [153], while in case of doubt the presence of tone was checked for by a few drops of sodium nitroprusside (SNP, $10^{-5} \mathrm{M}$; Sigma) on the preparation. During the equilibration period, a schematic diagram was drawn to identify sites for data collection. FITC-labeled 70 kDa dextrans (Dex-70; Sigma) and Texas Red labeled 40 kDa dextrans (Dex-40; Invitrogen) were intravenously injected as a bolus of $0.1 \mathrm{ml}$ at a concentration of $10 \mathrm{mg} / \mathrm{ml}$ in saline. Previous intravital microscopic studies have shown that under control conditions Dex-70 is significantly excluded from the endothelial surface in capillaries by the glycocalyx, whereas Dex-40 appears to have unimpaired access to the entire glycocalyx domain $[110,158]$. Cremaster capillaries from different microscopic fields were randomly chosen for examination and recorded on videotape using transand epi-illumination. Trans-illumination was used to determine endothelial surface position and velocity, flux, and width of RBCs. Epi-illumination was used to determine the distribution of Dex-70 and Dex-40, and was completed within a few seconds to minimize light-dye damage $[157,158]$. After baseline recordings were made $(20-30$ min), bradykinin (BK, $10^{-5} \mathrm{M}$; Sigma) or sodium nitroprusside (SNP, $10^{-6} \mathrm{M}$; Sigma) were randomly administered to the superfusate, and recordings of the same capillaries made, starting after $10 \mathrm{~min}$. After $30 \mathrm{~min}$, the vasodilator was washed out, the cremaster allowed to recover for $60 \mathrm{~min}$, and baseline recordings again made. Finally, the other vasodilator was added to the superfusate, and recordings repeated during this condition for 20-30 min. During baseline and administration of each vasodilator, proximal $1^{\text {st }}$ or $2^{\text {nd }}$ order arterioles were recorded as well for monitoring of arteriolar dilation (as percentage of baseline) in response to BK and SNP. 
Agonist induced increase in hematocrit and impaired glycocalyx barrier properties

\section{Data analysis}

Video images were digitized using a frame grabber (DT3152, PCI Local Bus) and image analysis software (Image-Pro Plus version 3.0, Media-Cybernetics, Silver Springs, PA, USA). Arteriolar diameter was measured from the distance between digital callipers positioned at the arteriolar wall. Capillary anatomic diameter and width of RBCs were measured from trans-illumination images by positioning digital callipers at the inside of the capillary wall and at the RBC border, respectively.

The glycocalyx RBC exclusion zone was determined by subtracting RBC width from capillary anatomic diameter and dividing the difference by two $[110,121,157,158]$. Glycocalyx exclusion zones for both dextrans were determined from epi-illumination images by measuring the width of the dye column with callipers, and subtracting this from the width of the capillary anatomic diameter, and dividing the difference by two $[110,121,157,158]$.

Capillary tube hematocrit (Hct, in \%) was calculated from measurements of capillary anatomic diameter (Dc, in $\mu \mathrm{m}$ ), flux of RBCs (F, in cells/s), and velocity of RBCs $(\mathrm{V}$, in $\mu \mathrm{m} / \mathrm{s})$ in each capillary, using the formula $[20,124]$ :

$$
H c t=\left[\frac{F}{V \pi \frac{1}{4} D c^{2}}\right] \times M C V
$$

where MCV is the mean corpuscular volume of RBCs in mice $\left(44 \mu \mathrm{m}^{3}\right)$ [96]. F was determined during slow-motion video playback from the time required for at least 50 RBCs to pass through a reference point inside the capillary. V was determined by measuring the length of a capillary segment and dividing it by the time required for RBCs to traverse this segment. The individual boundaries of RBCs could not be determined at high capillary velocities due of the limited video rate, and as a result capillaries with $\mathrm{V}>500 \mu \mathrm{m} / \mathrm{s}$ were discarded from the analysis. Capillaries were grouped based on RBC velocity of $<50,50-100, \ldots, 450-500 \mu \mathrm{m} / \mathrm{s}$ [157], because of the often observed correlation between red blood cell velocity and capillary tube hematocrit $[25,29,74,124]$.

Data are presented as means \pm SEM with $\mathrm{N}$ referring to the number of vessels or animals studied. In both animal groups, effects of BK or SNP were tested against baseline, or against the other vasodilator, using Students paired t-tests. Differences 
Chapter 3

between $\mathrm{C} 57 \mathrm{Bl} / 6$ and ApoE3-Leiden mice were tested using Students two sample t-tests. Results were considered statistically significant with $\mathrm{P} \leq 0.05$.

\section{Results}

Mean arterial pressure and heart rate were $60-70 \mathrm{mmHg}$ and $300-400 \mathrm{BPM}$ during experiments and did not change during respective treatments. Systemic hematocrit was $42.2 \pm 0.8 \%$ in $\mathrm{C} 57 \mathrm{Bl} / 6$ mice and $40.7 \pm 1.3 \%$ in ApoE3-Leiden mice.

\section{Arteriolar responses}

In control mice, $\mathrm{BK}$ and $\mathrm{SNP}$ increased arteriolar $(\mathrm{N}=10)$ diameter to $125 \pm 2 \%$ $(\mathrm{P}<0.05)$ and $128 \pm 2 \%(\mathrm{P}<0.05)$ of baseline $(36.2 \pm 1.5 \mu \mathrm{m})$, respectively. In ApoE3Leiden mice, arteriolar $(\mathrm{N}=10)$ diameter was increased from a baseline of $36.3 \pm 1.1$ $\mu \mathrm{m}$ to $116 \pm 1 \%(\mathrm{P}<0.05)$ and $131 \pm 2 \%(\mathrm{P}<0.05)$ during BK and SNP, respectively. Arteriolar dilation in ApoE3-Leiden mice during BK was reduced compared to SNP in the same animals $(\mathrm{P}<0.05)$ and compared to dilation during $\mathrm{BK}$ in $\mathrm{C} 57 \mathrm{Bl} / 6$ mice $(\mathrm{P}<0.05)$.

\section{Capillary responses}

\section{Control mice}

Responses were measured in $\mathrm{N}=71$ capillaries of control C57Bl/6 mice. Capillary anatomic diameter was $4.9 \pm 0.1 \mu \mathrm{m}$ during baseline and did not change after vasodilator administration. RBC flux was $11.2 \pm 0.7$ cells/s during baseline, and 36.1 \pm 2.2 cells $/ \mathrm{s}(\mathrm{P}<0.05)$ and $35.4 \pm 2.2$ cells $/ \mathrm{s}(\mathrm{P}<0.05)$ during $\mathrm{BK}$ and $\mathrm{SNP}$, respectively. RBC velocity increased from $291 \pm 15 \mu \mathrm{m} / \mathrm{s}$ during baseline to $408 \pm 20 \mu \mathrm{m} / \mathrm{s}$ $(\mathrm{P}<0.05)$ during superfusion of $\mathrm{BK}$ and to $423 \pm 16 \mu \mathrm{m} / \mathrm{s}$ during $\mathrm{SNP}(\mathrm{P}<0.05)$. Capillary tube hematocrit varied from $7.1 \pm 1.7 \%$ at high $\mathrm{RBC}$ velocities to $14.8 \pm$ $1.3 \%$ at low velocities during baseline conditions (figure 3.1); on average, capillary tube hematocrit was $8.7 \pm 0.3 \%$. Tube hematocrit increased during administration of BK $(21.2 \pm 1.2 \% ; \mathrm{P}<0.05)$ and SNP $(22.2 \pm 0.9 \% ; \mathrm{P}<0.05)$. During vasodilator administration, the relation between capillary tube hematocrit and $\mathrm{RBC}$ velocity was 
Agonist induced increase in hematocrit and impaired glycocalyx barrier properties

shifted in a parallel manner, resulting in a higher tube hematocrit at a given RBC velocity compared to baseline (figure 3.1). Capillary tube hematocrit and red blood cell velocity were not different after washout of the $1^{\text {st }}$ vasodilator compared to before vasodilator administration.

\section{C57BI/6 mice}

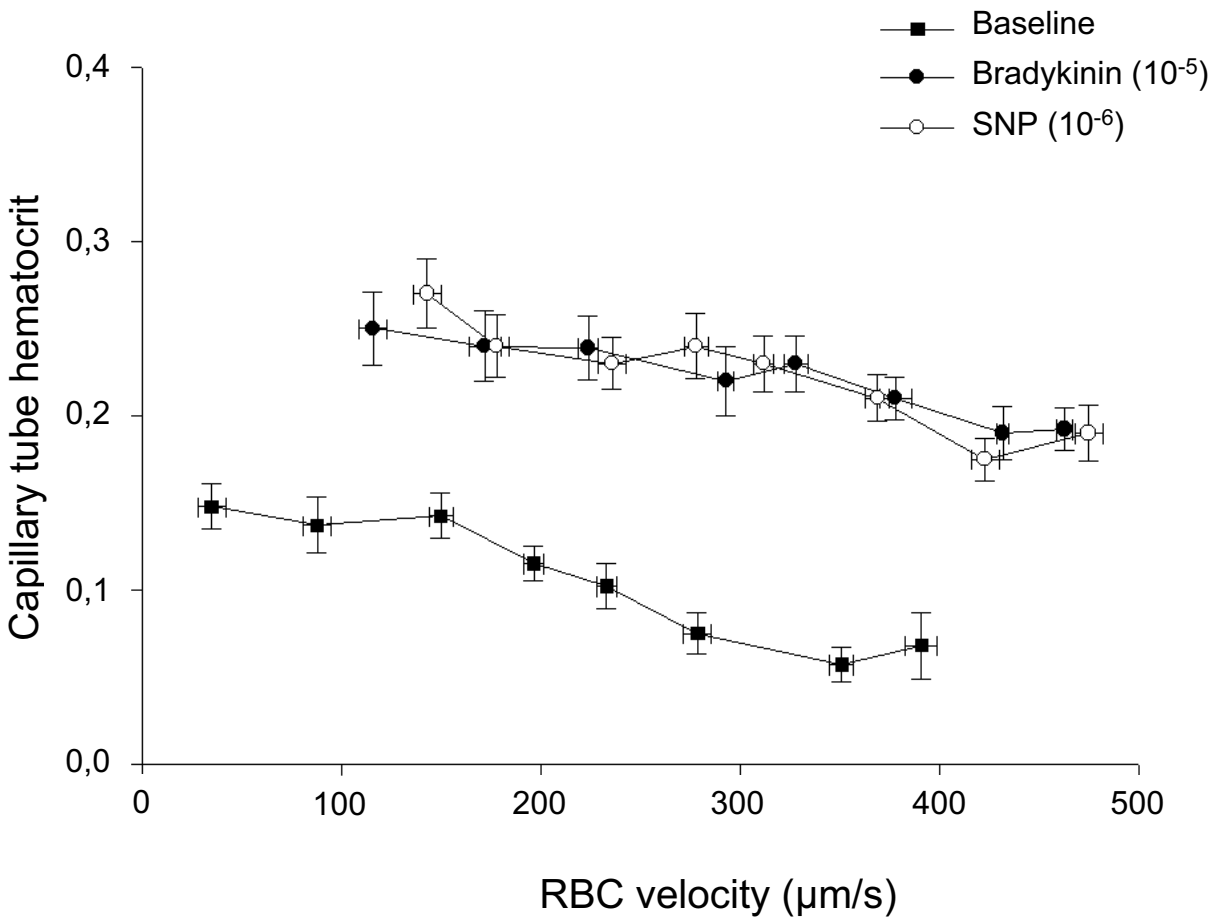

Figure 3.1 BK and SNP increase capillary tube hematocrit and RBC velocity in control mice. Capillary tube hematocrit decreases with increasing RBC velocity, during baseline and in the presence of vasodilator. Vasodilator administration is associated with an increased capillary tube hematocrit for any given RBC velocity.

In $\mathrm{C} 57 \mathrm{Bl} / 6$ mice, exclusion of RBCs from the endothelium was $0.55 \pm 0.02 \mu \mathrm{m}$ during baseline conditions, and not changed after administration of $\mathrm{BK}(0.55 \pm 0.01 \mu \mathrm{m})$ or SNP $(0.57 \pm 0.01 \mu \mathrm{m})$. In contrast, the Dex-70 exclusion zone, which was $0.37 \pm 0.01$ $\mu \mathrm{m}$ during baseline, was significantly reduced during superfusion of BK $(0.17 \pm 0.01$ $\mu \mathrm{m} ; \mathrm{P}<0.05)$ and SNP $(0.15 \pm 0.01 \mu \mathrm{m} ; \mathrm{P}<0.05)$. The exclusion zone for Dex-70 
after washout of the $1^{\text {st }}$ vasodilator and before application of the $2^{\text {nd }}$ was $0.38 \pm 0.01$ $\mu \mathrm{m}$, and not different from baseline values. These discrepant effects of BK and SNP on the exclusion of RBCs versus that of the dye are further exemplified in figure 3.2 and 3.3, which shows the exclusion zone for RBCs (figure 3.2) and Dex-70 (figure 3.3) for different RBC velocities during baseline and vasodilator administration. Exclusion zones appeared independent of $\mathrm{RBC}$ velocity in the range of $100-500 \mu \mathrm{m} / \mathrm{s}$. Below $100 \mu \mathrm{m} / \mathrm{s}$, red blood cell exclusion seemed to be impaired (figure 3.2). The exclusion zone of Dex-40 was $0.07 \pm 0.02 \mu \mathrm{m}$ during baseline conditions, and not changed after administration of $\mathrm{BK}(0.01 \pm 0.02 \mu \mathrm{m})$ or $\mathrm{SNP}(0.03 \pm 0.01 \mu \mathrm{m})$.

\section{C57BI/6 mice}

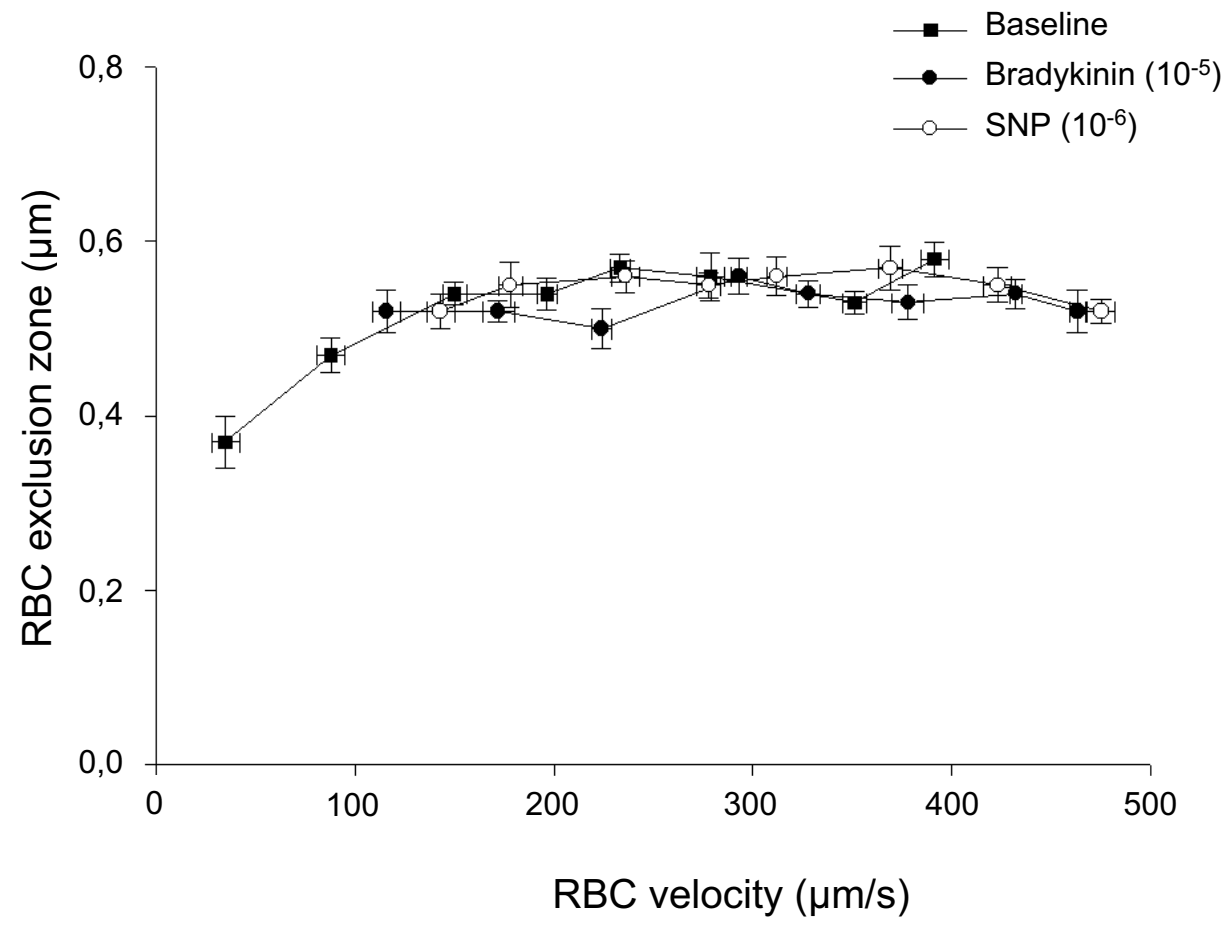

Figure 3.2 RBC exclusion zone, used as measure of glycocalyx thickness, decreases at RBCs velocities $<100 \mu \mathrm{m} / \mathrm{s}$, and is not affected by BK and SNP. 


\section{C57BI/6 mice}



Figure 3.3 Dex-70 exclusion zone is independent of RBC velocity, and decreases during vasodilator administration.

\section{Hyperlipidemic mice}

In ApoE3-Leiden mice, the majority of capillaries $(\mathrm{N}=55)$ were found to present subendothelial lipid deposits and to have impaired glycocalyx exclusion properties (see below). The other capillaries $(\mathrm{N}=10)$ were without signs of lipid deposits and demonstrated normal (i.e., comparable to control mice) exclusion of RBCs and Dex-70 from the vessel wall. Because we were interested in the effect of vasodilator modulation during a condition of glycocalyx degradation, only those capillaries with subendothelial lipid deposits are presented here. Anatomic diameter of these capillaries was 5.1 $\pm 0.1 \mu \mathrm{m}$ during baseline and not different during vasodilator administration. RBC 
flux was higher $(\mathrm{P}<0.05)$ than in control mice, $23.1 \pm 1.7$ cells $/ \mathrm{s}$ during baseline, and $41.3 \pm 1.2$ cells $/ \mathrm{s}(\mathrm{P}<0.05$ vs baseline $)$ and $39.4 \pm 2.0$ cells $/ \mathrm{s}(\mathrm{P}<0.05)$ during $\mathrm{BK}$ and $\mathrm{SNP}$, respectively, while RBC velocity increased from $225 \pm 16 \mu \mathrm{m} / \mathrm{s}$ during baseline to $382 \pm 18 \mu \mathrm{m} / \mathrm{s}(\mathrm{P}<0.05)$ during superfusion of $\mathrm{BK}$ and to $364 \pm 17 \mu \mathrm{m} / \mathrm{s}$ during SNP $(\mathrm{P}<0.05)$. Figure 3.4 indicates that for a given $\mathrm{RBC}$ velocity, capillary tube hematocrit did not change after vasodilator administration. Thus, average capillary tube hematocrit was $23 \pm 0.8 \%$ during baseline conditions $(\mathrm{P}<0.05$ vs $\mathrm{C} 57 \mathrm{Bl} / 6)$, and not affected during administration of BK $(24.3 \pm 1.1 \%)$ and $\operatorname{SNP}(22.7 \pm 1.4 \%)$.

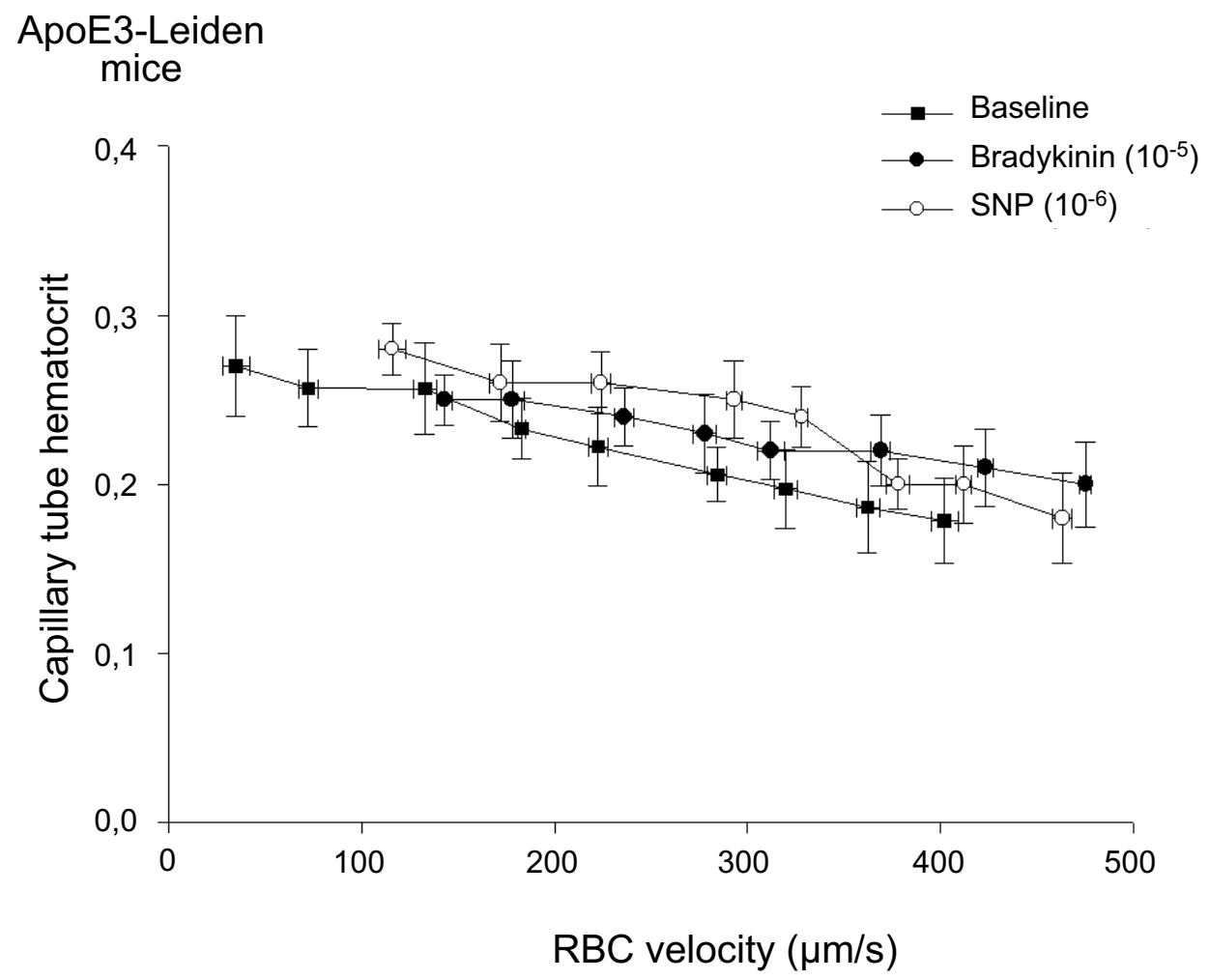

Figure 3.4 Lack of effect of BK and SNP on capillary tube hematocrit in hyperlipidemic mice. In capillaries with lipid deposits of ApoE3-Leiden mice, capillary tube hematocrit decreases with increasing RBC velocity, during baseline and in the presence of vasodilator. Vasodilator administration does not affect capillary tube hematocrit for any given RBC velocity. 
Agonist induced increase in hematocrit and impaired glycocalyx barrier properties

In the capillaries with subendothelial lipid deposits, the exclusion zone of RBCs from the endothelium increased from $0.15 \pm 0.02 \mu \mathrm{m}$ at velocities $<50 \mu \mathrm{m} / \mathrm{s}$ to $0.33 \pm 0.02$ $\mu \mathrm{m}$ at velocities $>450 \mu \mathrm{m} / \mathrm{s}$ (figure 3.5 ), illustrating a reduction in glycocalyx exclusion over the entire range of $\mathrm{RBC}$ velocities compared to the control $\mathrm{C} 57 \mathrm{Bl} / 6$ mice (figure 3.2). On average, RBC exclusion was $0.30 \pm 0.02 \mu \mathrm{m}$ during baseline conditions $(\mathrm{P}<0.05$ vs $\mathrm{C} 57 \mathrm{Bl} / 6)$, and not changed after administration of BK or SNP. The Dex-70 exclusion zone was only $0.05 \pm 0.02 \mu \mathrm{m}$ during baseline $(\mathrm{P}<0.05$ vs $\mathrm{C} 57 \mathrm{Bl} / 6)$, not dependent on RBC velocity (figure 3.6) and not changed during superfusion of $\mathrm{BK}(0.10 \pm 0.02 \mu \mathrm{m})$ and SNP $(0.04 \pm 0.02 \mu \mathrm{m})$. The exclusion zone of Dex-40 was $0.02 \pm 0.03 \mu \mathrm{m}$ during baseline conditions, and not changed after administration of $\mathrm{BK}(0.04 \pm 0.02 \mu \mathrm{m})$ or $\operatorname{SNP}(0.01 \pm 0.02 \mu \mathrm{m})$.

\section{Discussion}

The endothelial glycocalyx has been hypothesized to significantly reduce perfused capillary volume under control conditions by excluding flowing plasma and RBCs from a relatively thick region near the luminal endothelial surface. The present study shows that bradykinin (BK) and sodium nitroprusside (SNP) increase capillary tube hematocrit about 2.5-fold in cremaster capillaries of healthy $\mathrm{C} 57 \mathrm{Bl} / 6$ mice. This increase was paralleled by an increased accessibility of the glycocalyx domain for FITC-labeled $70 \mathrm{kDa}$ dextrans without a change in RBC exclusion. BK and SNP had no effect on tube hematocrit or glycocalyx barrier properties in capillaries of hyperlipidemic ApoE3-Leiden mice that showed signs of glycocalyx degradation. Our data suggest that vasodilator substances can increase functionally perfused capillary volume by modulation of glycocalyx exclusion.

\section{Glycocalyx degradation in hyperlipidemic mice}

To test whether vasodilator effects on capillary hemodynamics and intravascular tracer distribution pertained to properties of the glycocalyx, we chose the high fat-fed Apo3Leiden mouse as model for atherogenic glycocalyx degradation. Our rationale for this was that we wanted to avoid transient effects and time consuming infusions that are intrinsic to the use of glycocalyx degrading enzymes [29,47, 150,154], while we had 


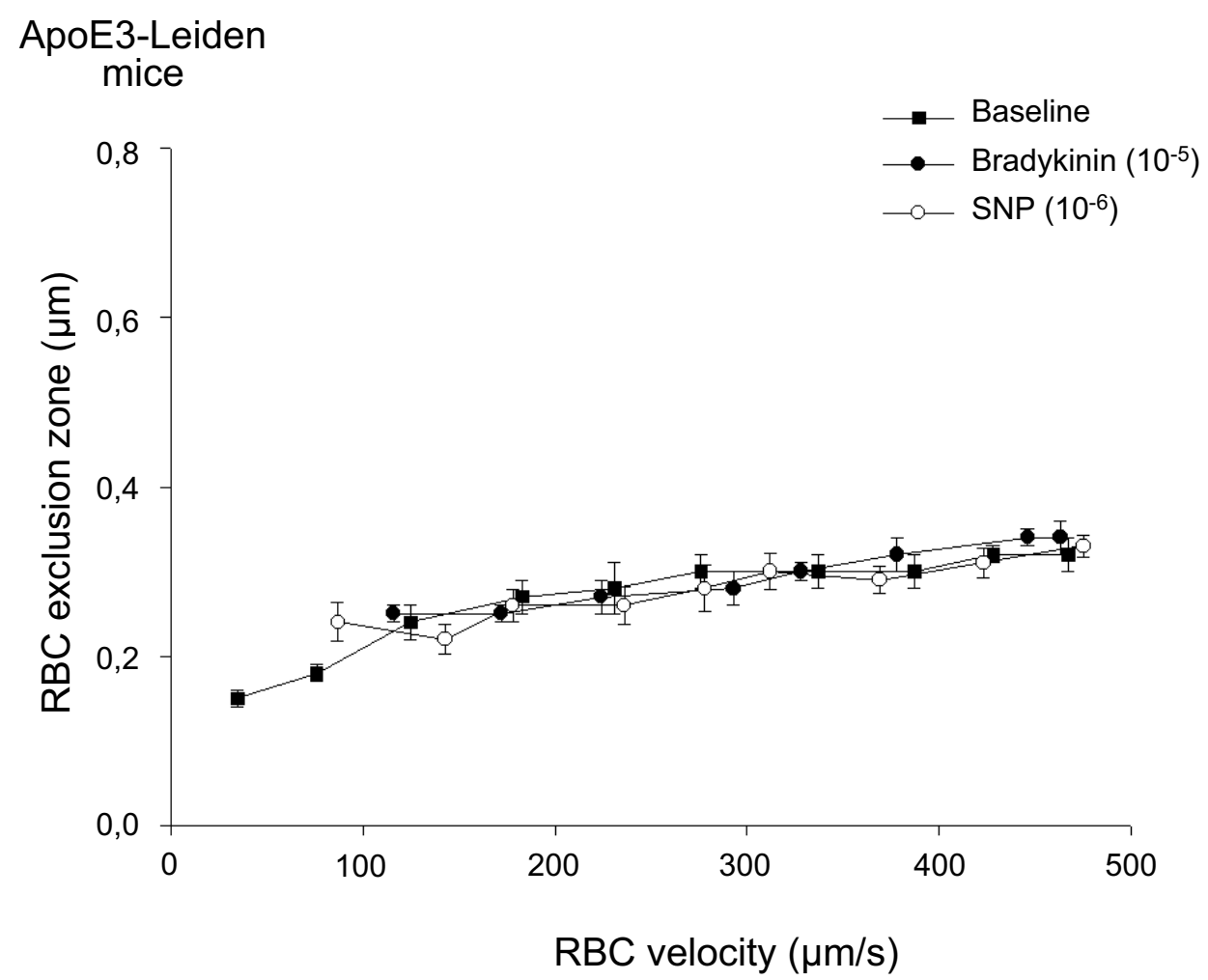

Figure 3.5 Glycocalyx exclusion is not changed during vasodilator superfusion in hyperlipidemic mice. In capillaries with lipid deposits in ApoE3-Leiden mice, RBC exclusion zones was profoundly reduced during baseline compared to control mice (figure 3.2), yet not affected by administration of BK and SNP.

previously demonstrated the potency of glycocalyx degradation by an atherogenic stimulus, in this case bolus infusion of oxidized LDL [20]. The majority of capillaries in the hyperlipidemic mice indeed demonstrated a greatly reduced RBC exclusion under baseline conditions (figure 3.5), and the exclusion zone of $\sim 0.30 \mu \mathrm{m}$ is very comparable with the reported $\mathrm{RBC}$ exclusion zone of $0.30-0.35 \mu \mathrm{m}$ after the bolus of oxidized LDL [20]. In addition, the decrease in RBC exclusion after oxidized LDL was paralleled by an increase in capillary tube hematocrit towards $30-35 \%$ and an increase in RBC flux of 150-200\% without a change in RBC velocity [20], and this is very 


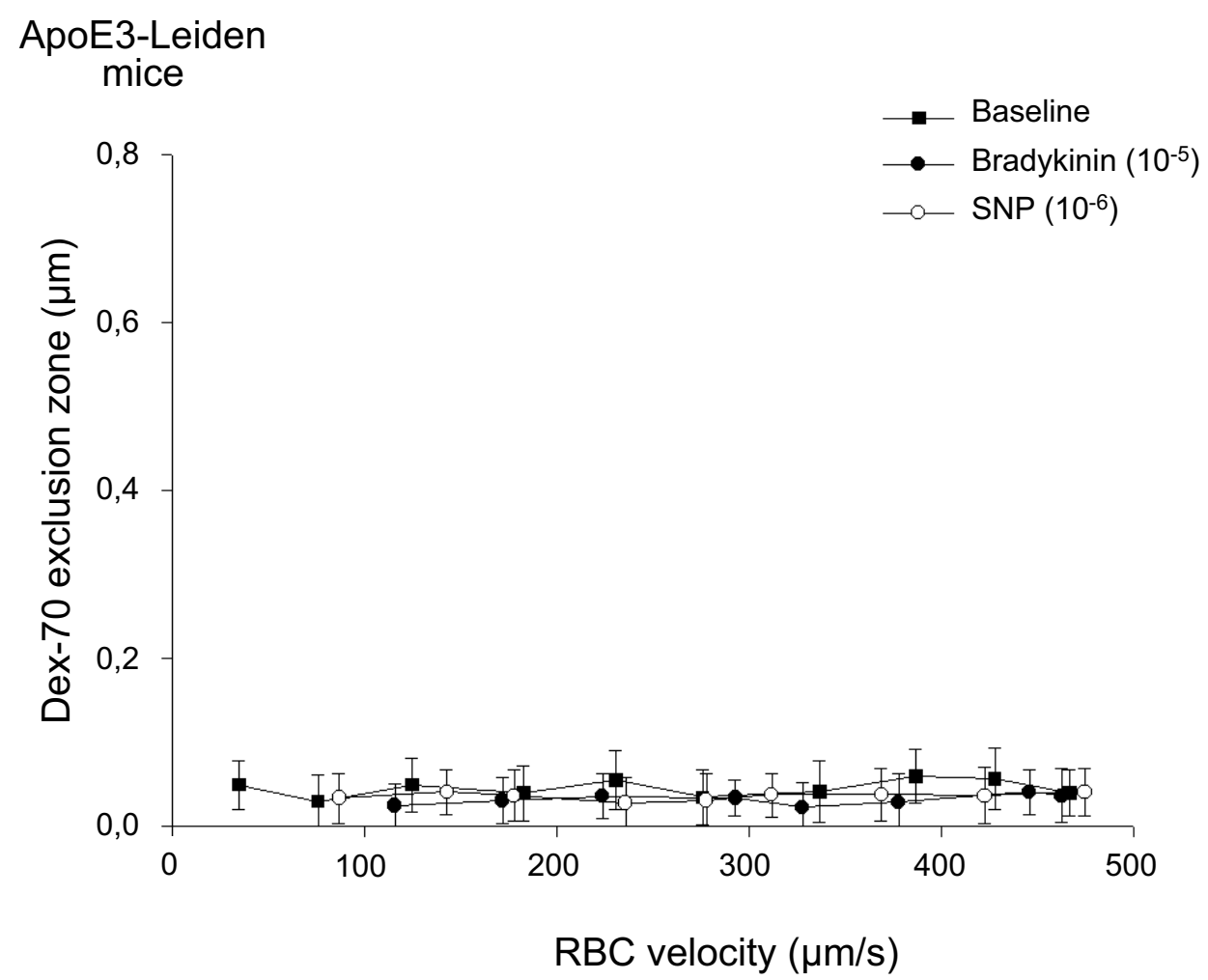

Figure 3.6 Glycocalyx exclusion is not changed during vasodilator superfusion in hyperlipidemic mice. In capillaries with lipid deposits in ApoE3-Leiden mice, Dex-70 exclusion zones was profoundly reduced during baseline compared to control mice (figure 3.3), yet not affected by administration of BK and SNP.

similar to the current hemodynamic observations in ApoE3-Leiden capillaries. Of note, the ApoE3-Leiden mice had not exclusively signs of glycocalyx degradation, and the chronic atherogenic conditions unquestionably impacted on many additional pathways related to vascular homeostasis as well as rheology. Thus, these animals develop severe hyperlipidemia and atherosclerosis after 3 months on a high-fat/high-cholesterol diet [147], while our own results indicate impairment of vascular barrier properties by the presence of subendothelial lipid deposits and endothelial dysfunction by the impaired arteriolar dilation to BK (present data) and to reactive hyperemia [153]. In 
addition, the hyperlipidemic conditions per se might, by an increased plasma viscosity, have influenced microcirculatory perfusion characteristics in the ApoE3-Leiden mice.

\section{Vasodilator effects on capillary perfusion}

Marked increases in capillary tube hematocrit and red blood cell velocity during muscle contractions and adenosine were originally reported by Klitzman and Duling [74]. It was hypothesized that the changes in capillary tube hematocrit were related to metabolic and vasoactive modulation of the contribution of a $1.2 \mu \mathrm{m}$-thick slow-moving plasma layer to functional capillary perfusion. Ten years later it was indicated that this layer was represented by the endothelial glycocalyx, since enzymatic treatment of glycocalyx structures with heparinase was associated with a doubling in baseline capillary hematocrit [29].

In line with reports in the literature $[28,29,74,124]$, average capillary tube hematocrit in healthy capillaries was low under control conditions, about $20 \%$ of systemic hematocrit. Capillary tube hematocrit, the fractional volume of a capillary occupied by RBCs, was derived from capillary anatomical diameter and the velocity and flux of RBCs in the capillary, measurements that all have potential errors associated with them. Nevertheless, this estimate has been used in many previous studies and the possible error associated with the calculation is regarded to be small: Sarelius \& Duling estimated the standard deviation of the hematocrit determination to be $\pm 17 \%$ [124]. Furthermore, RBCs could not individually be distinguished in the present study at velocities $>500 \mu \mathrm{m} / \mathrm{s}$ (see Methods). This was, however, the case in only $\sim 5 \%$ of the capillaries and neglecting these data in the analysis did therefore not have a significantly impact on the results.

Under control conditions, capillary tube hematocrit increased with decreasing red blood cell velocity $\left(\mathrm{V}_{r b c}\right)$ (figure 3.1 ), and the decrease in $\mathrm{RBC}$ exclusion at velocities $<200 \mu \mathrm{m} / \mathrm{s}$ (figure 3.2) seems to underlie this at least for the lower velocity range. This behaviour can be accounted for by glycocalyx deformation by expansion of the RBCs at low velocities, as was experimentally shown by Vink and Duling [157], and argues against a shear-dependent compression of the glycocalyx [115]. An increased red blood cell exclusion with increasing velocity was also predicted by the model of Secomb et al. on the motion of red blood cells in a glycocalyx-lined capillary using lubrication theory [127]. This inverse relation between red blood cell velocity and cap- 
Agonist induced increase in hematocrit and impaired glycocalyx barrier properties

illary tube hematocrit seems at first sight in contrast to the early studies from Duling and coworkers in which a positive relation between red blood cell velocity (and arteriolar diameter) on the one hand and capillary tube hematocrit on the other hand was observed $[25,29,74,124]$. In those studies, changes in red blood cell velocity evoked by addition of oxygen or adenosine in the superfusate were associated with concomitant changes in capillary tube hematocrit, suggesting a role for hemodynamic effects in RBC occupation of the capillary. Similarly, BK and SNP superfusion evoked arteriolar dilations and increases in red blood cell velocity and capillary tube hematocrit in the current experiments. However, our data show that the relation between capillary tube hematocrit and $\mathrm{V}_{r b c}$ was actually shifted in a more or less parallel manner during vasodilator administration (figure 3.1). In the hyperlipidemic mice, a similar shift was observed during baseline conditions already (figure 3.4), which agrees with the observation of Desjardins \& Duling and Constantinescu et al. that heparinase or oxidizedLDL treatment doubled baseline capillary tube hematocrit in the face of a constant red blood cell velocity $[20,29]$. In a recent study in which capillary hemodynamics in rat spinotrapezius muscle were studied during recovery from muscle contractions, a relation between changes in red blood cell velocity and capillary tube hematocrit was absent as well [33], illustrating that also under these physiologically relevant conditions capillary tube hematocrit is a parameter that can change independently from a change in red blood cell velocity. Further arguing against a simple hemodynamic effect of the vasodilators on capillary tube hematocrit is the observation that administration of BK or SNP in the ApoE3-Leiden animals increased red blood cell velocity but did not affect the relation between capillary tube hematocrit and red blood cell velocity (figure 3.4). In line herewith, Desjardins and Duling showed that glycocalyx treatment with heparinase obliterated the relationship between red blood cell velocity and capillary tube hematocrit in response to oxygen or adenosine supplementation to the superfusate [29]. These findings altogether indicate the existence of an inverse relation between capillary tube hematocrit and $\mathrm{V}_{r b c}$; this relation seems relevant for different "states" of the glycocalyx (i.e., baseline, vasodilator, degradation). 


\section{Agonist-induced increases in capillary tube hematocrit: under- lying mechanisms}

Differences in mean red blood cell velocity compared to mean plasma velocity cause a reduction in tube hematocrit compared to systemic hematocrit $[36,79]$. The lower limit attributed to this "single vessel Fåhraeus effect" has been postulated to be a $50 \%$ reduction in capillary tube hematocrit compared to systemic hematocrit [36], while a reduction beyond this value might be explained by the presence of an immobilized plasma volume represented by the glycocalyx $[29,74,126,157]$, or to be caused by microvascular network events, such as phase separation of RBCs and plasma at upstream bifurcations, or intercapillary heterogeneity of blood flow ("network Fåhraeus effect"; $[36,112])$. The network Fåhraeus effect can be altered according to perfusion heterogeneity, where less heterogeneity would lead to higher capillary tube hematocrits. A reduction in heterogeneity of capillary red blood cell velocity with vasodilatation or increased flow [140] has not consistently been observed, however [26, 27], and with respect to our own results, the increase in red blood cell velocity during SNP or bradykinin did not seem to coincide with a robust reduction in perfusion heterogeneity in both control and hyperlipidemic animals, since the standard error of red blood cell velocity increased during vasodilator administration compared to baseline as well (see Results).

Therefore, we like to consider also the possibility that increases in glycocalyx accessibility for flowing plasma are underlying observed increases in capillary tube hematocrit towards $50 \%$ of systemic hematocrit. In the hyperlipidemic mice, the ratio between capillary tube hematocrit $(23 \%)$ and systemic hematocrit $(41 \%)$ was close to 0.5 , suggesting that in these capillaries the entire anatomic volume of the capillary was available for perfusion, and that plasma retardation by the glycocalyx was irrelevant. Indeed, there was barely any exclusion of $70 \mathrm{kDa}$ dextrans in these capillaries (figure 3.6), while the $0.30 \mu \mathrm{m}$ thick exclusion zone of RBCs might represent the lower limit for RBCs approaching the endothelium and reflect a plasma lubrication layer that is required for unimpeded motion of $\mathrm{RBCs}$ in capillaries [126,157].

An important question is to which extent the effective glycocalyx thickness needs to be diminished to obtain values of capillary tube hematocrit that are half of the systemic values during vasodilator administration in the control animals. On the basis of a sim- 
Agonist induced increase in hematocrit and impaired glycocalyx barrier properties

ple two-compartment model with RBCs and plasma flowing in the central core of the capillary and an additional immobilized plasma layer near the vessel wall representing the glycocalyx $[29,74]$, an increase in perfused diameter of $1.8 \mu \mathrm{m}$ in a capillary with an anatomical diameter of $4.9 \mu \mathrm{m}$ would explain the 2.5 fold increase in capillary tube hematocrit during BK and SNP, agreeing with a reduction in blood-excluding glycocalyx thickness of $0.9 \mu \mathrm{m}$ from baseline to vasodilator conditions. This value is comparable with the original estimations of the slow-moving plasma layer by Duling and coworkers [29,74], but significantly larger than the exclusion zones in the current and previous studies as well as $\mu$-PIV measures, all indicating dimensions of the glycocalyx in cremaster vessels that range from $\sim 0.2$ to $0.6 \mu \mathrm{m}[47,110,121,129,157,162]$. This disparity calls for a prudent interpretation of the vasodilator-associated increases in capillary tube hematocrit on the basis of a change in effective glycocalyx thickness alone. On the other hand, estimations to explain the discrepancy between experimental estimates of apparent viscosity or flow resistance in microvessels in vitro versus in vivo by Pries and coworkers have generally yielded values of $>1 \mu \mathrm{m}$ for effective glycocalyx thickness $[113,115]$, while in isolated small mesenteric arteries the exclusion zone for FITC-labeled $150 \mathrm{kDa}$ dextrans was estimated to be $2-3 \mu \mathrm{m}$ [144]. The basis for this discrepancy is unknown at the moment and inaccuracies in both the experimental measurements (see below) and the theoretical models used might underlie this difference.

\section{Vasodilator effects on glycocalyx exclusion properties: possible mechanisms}

Consistent with previous measurements of anionic dextran distribution in rodent capillaries $[47,110,121,157,158]$, we observed a significant intravascular zone to which FITC-labeled $70 \mathrm{kDa}$ dextrans had no access during control conditions (figure 3.3). The exclusion zone for the dextrans was $0.35-0.40 \mu \mathrm{m}$, which agrees with previous studies showing glycocalyx exclusion of FITC-labeled $70 \mathrm{kDa}$ dextrans to be about $50 \%$ of RBC exclusion $[47,110,121,157]$. The larger dextran distribution volume compared to the RBCs seems to indicate that the dextrans partly permeate into the glycocalyx domain during resting conditions [150], and as a result the endothelium-dextran gap likely provides an underestimation of true blood excluding glycocalyx dimensions. 
Interestingly, the endothelium-dextran gap was found to be $\sim 1 \mu \mathrm{m}$ when RBCs were absent in the capillary [159], and we like to consider, therefore, the possibility that the current measurements of both endothelium-RBC and endothelium-dextran dye gap are underestimating the effective solute excluding glycocalyx volume because of passing RBCs partly compressing the glycocalyx. In the case there is ample time for recovery of glycocalyx dimensions in between successive red blood cells, i.e., at the low $\mathrm{RBC}$ fluxes that are occurring during baseline, the actual glycocalyx thickness might be thicker as what is expected based on our measurement of the endothelium-RBC or -dye gap across the length of the observed capillary. Recovery of glycocalyx thickness in response to an almost complete glycocalyx compression by passing leukocytes was estimated to follow a single exponential with a time characteristic $(\tau)$ of 0.38 s $[157,163]$, but recovery after partial compression by passing red blood cells might be faster. Effective glycocalyx thickness is then a result of true physical glycocalyx thickness, recovery time, and red blood cell flux. For example, in the case of a $1.5 \mu \mathrm{m}$ thick glycocalyx, a $\tau$ of $0.22 \mathrm{~s}$ will be sufficient to get to an effective thickness of the layer of $0.9 \mu \mathrm{m}$ when red blood cells are passing every $0.1 \mathrm{~s}$ (i.e., a RBC flux of $10 / \mathrm{s}$ ); in the case of a true thickness of $1.0 \mu \mathrm{m}$, a $\tau$ of $0.07 \mathrm{~s}$ is needed for RBCs passing every $0.1 \mathrm{~s}$.

During BK and SNP, the exclusion of Dex-70 was impaired with $\sim 0.15 \mu$ m while RBC exclusion was not altered (figure 3.2). The disparity in the effect of a certain stimulus on macromolecule exclusion versus that on RBC exclusion has been noticed in previous studies from Duling and coworkers as well $[47,110,121]$. Thus, increases in FITClabeled dextran accessibility without changes in RBC exclusion were also observed in cremaster vessels during ischemia-reperfusion and hyaluronidase treatment. In the case of adenosine, Platts \& Duling showed a profound reduction in Dex-70 exclusion at the micromolar concentration of adenosine, while a small reduction in RBC exclusion appeared at a 100-fold higher dose only [110]. An increased dextran accessibility in the face of an unchanged RBC exclusion has been interpreted as a manifestation of a more open or porous structure of the glycocalyx $[47,110,121]$. Glycocalyx composition and dimensions are the ultimate result of continuous biosynthesis of polysaccharide structures and association with blood-borne proteins on one hand and shedding or release of components on the other hand, while the actual dynamic state of the glycocalyx depends on the intrinsic properties of its constituents, their interactions, and 
Agonist induced increase in hematocrit and impaired glycocalyx barrier properties

the local microenvironment, such as cation content and $\mathrm{pH}[93,136,162]$. Any change in production, shedding, and local conditions may therefore affect the permeation of $70 \mathrm{kDa}$ dextrans into the glycocalyx domain.

Our results suggest a role for NO in the impairment of glycocalyx barrier properties by bradykinin and SNP. There is evidence that NO is capable of degrading heparan sulfates [155], but this process seems too slow to explain the observed dynamics of changes in capillary tube hematocrit and glycocalyx exclusion. The pilot studies of Duling showed moment-to-moment variations in capillary tube hematocrit to occur in the face of a constant discharge hematocrit, suggesting that glycocalyx exclusion properties may change very rapidly [28]. Platts and Duling found glycocalyx exclusion to be impaired at their first observation point, which occurred 6 min after adenosine administration had started [110], while our previous study in dog hearts suggests that adenosine-induced glycocalyx modulation may occur almost instantaneously [154], at least in the time frame of maximal coronary hyperemia in response to a bolus of adenosine ( $\sim 15 \mathrm{~s})$. As an alternative for degradation of polysaccharide structures by NO, we like to propose a change in charge density of the glycocalyx as possible mechanism by which NO can rapidly change its properties. Our group previously demonstrated in small arteries of the rat mesentery that the glycocalyx can move from a collapsed to an extended state as ionic strength of the superfusate decreases [145], and we envision that NO by scavenging of superoxide anions within the glycocalyx (e.g., produced by glycocalyx-bound xanthine oxidase [121]) might induce a similar effect. In addition, rapid reactions of NO effecting nitrosylation of albumin and other proteins [132] within the glycocalyx might alter their binding affinity for glycocalyx polysaccharide structures, thereby changing the structural barrier properties of the glycocalyx $[2,150]$. Altogether, these NO-mediated changes may convert the glycocalyx towards a more porous compartment with increased hydraulic and tracer conductivity without shedding of its solid glycosaminoglycan components. Theoretical models of red cell motion in a single file $[24,126]$ have indeed shown that with a constant layer thickness an increase in hydraulic conductivity of the glycocalyx can take place without significant changes in RBC shape and that this causes capillary tube hematocrit to increase. Of note, vasodilator administration in the control mice resulted in capillary tube hematocrits that were very close to those during baseline conditions in the hyperlipidemic mice, suggesting that in the presence of the vasodilators the whole anatomic volume 
of the capillary was available for perfusion, and that the entire glycocalyx domain was accessed by plasma flow. Dextran exclusion was, however, only modestly reduced to $\sim 40 \%$ of baseline, and a zone of $\sim 0.15 \mu \mathrm{m}$ thickness from which these molecules seemed to be excluded, remained (figure 3.3), illustrating that measures of $70 \mathrm{kDa}$ dextran distribution cannot be simply interpreted as correct measures of plasma distribution. Although an increase in hydraulic conductivity of the endothelial cell layer by bradykinin and SNP is expected to occur as well, this alone seems not sufficient to explain the increase in dextran permeation into the glycocalyx, because in the absence of a reduction in reflection coefficient of the dextran, a sole increase in hydraulic conductivity will result in a dilution of this fluorescent tracer within the glycocalyx. In contrast, we actually observed an increase in $70 \mathrm{kDa}$ dextran concentration in the glycocalyx compartment during vasodilator administration, demonstrating that the evoked impairment of tracer barrier properties surpassed the presumed reduction in hydraulic resistance.

\section{Glycocalyx modulation and regulation of capillary perfusion}

From our present data and the aforementioned data from Duling and coworkers, we like to propose the concept in which vasoactive substances can 'recruit' capillary volume for perfusion by increasing accessibility of the glycocalyx for flowing plasma, as reflected by the increased dextran accessibility, without necessarily changing glycocalyx dimensions [151]. Acting in parallel to relaxation of resistance vessels, this may enable coordination of substrate delivery (flow) and exchange (surface area and permeability) [151]. The potency of adenosine to 'recruit' glycocalyx volume in the coronary vascular bed was recently determined in canine hearts [154]. We compared the increase in coronary conductance during adenosine-induced hyperemia to the increase in conductance during reactive hyperemia and found that under control conditions adenosine increased conductance up to $40 \%$ more than reactive hyperemia [154]. The difference was, however, virtually abolished upon enzymatic treatment of the glycocalyx, which resulted in an increase in reactive hyperemia without changing adenosine hyperemia [154]. Together with the lack of effect of BK and SNP on capillary tube hematocrit and glycocalyx exclusion in the hyperlipidemic mice in the present study, the latter finding in the canine heart also suggests that the capability for agonistinduced modulation of the glycocalyx is substantially lost in case of glycocalyx per- 
Agonist induced increase in hematocrit and impaired glycocalyx barrier properties

turbation. Glycocalyx perturbation, as has been demonstrated during hyperglycemic and atherogenic conditions in patients and animal models [20,104,151], may accordingly be associated with an impairment of the ability of vasoactive substance to recruit glycocalyx volume, and together with a loss of shear mediated vasodilatation [150], contribute to a deterioration of perfusion regulation.

\section{Conclusion}

In conclusion, the present data show that both bradykinin and sodium nitroprusside administration onto cremaster muscles of healthy $\mathrm{C} 57 \mathrm{Bl} / 6$ mice robustly increase capillary tube hematocrit as well as the accessibility of the glycocalyx domain for FITC-labeled $70 \mathrm{kDa}$ dextrans. In contrast, vasodilator modulation of tube hematocrit and glycocalyx exclusion is abolished in capillaries with signs of glycocalyx degradation (hyperlipidemic mice). Our data suggest that vasodilator substances can increase functionally perfused capillary volume by effectively reducing glycocalyx exclusion. Modulation of blood excluding glycocalyx volume in capillaries by vasodilator substances in parallel to relaxation of resistance vessels may enable coordination of substrate delivery and exchange.

\section{Acknowledgment}

The authors would like to thank Prof. Dr. L. M. Havekes for granting permission to use ApoE3-Leiden mice in the current experiments. 



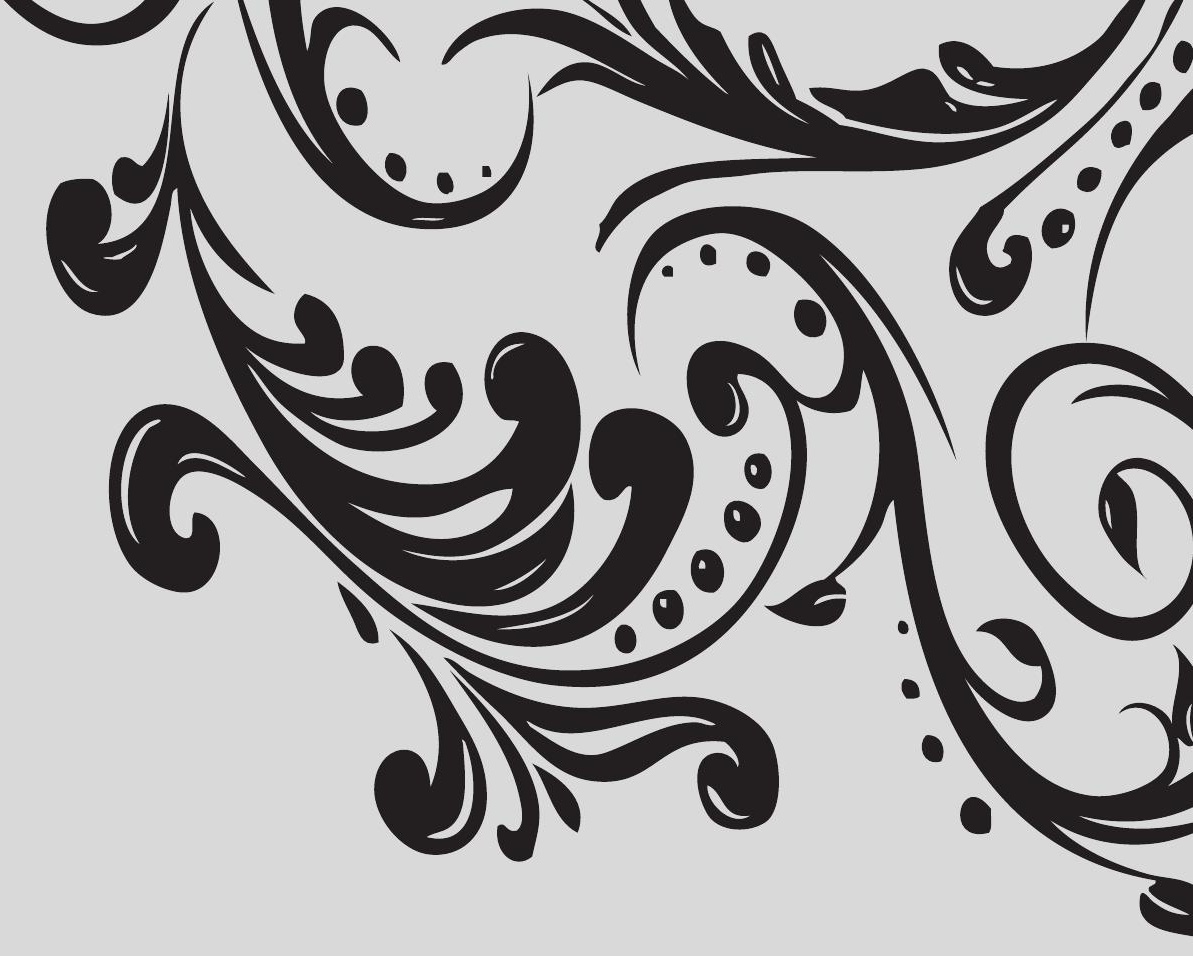

Systemic glycocalyx measurements 



\section{Acute hyaluronidase treatment reduced systemic glycocalyx volume in mice}

The contents of this chapter are based on Jurgen WGE Van Teeffelen, Judith Brands, Bart Eskens, Carin Jansen, Bernard van den Berg, Jos AE Spaan, and Hans Vink, Whole-body glycocalyx exclusion properties in mice, in preparation 


\section{Abstract}

The glycocalyx forms the interface between the flowing blood and the endothelium and has been indicated to play an important role in vascular homeostasis. Recently, whole-body glycocalyx volumes were measured in human subjects by determining the difference between circulating plasma volume, derived from the dilution of labeled red blood cells and large vessel hematocrit, versus the distribution volume of a glycocalyx accessible tracer, i.e. $40 \mathrm{kDa}$ dextrans (Dex-40). In the current study, we validated this measurement by determining the effect of hyaluronidase treatment on wholebody glycocalyx volume in anesthetized and ventilated $\mathrm{C} 57 \mathrm{Bl} / 6$ mice. In control mice, treated with saline, circulating plasma volume and Dex-40 volume were $0.72 \pm$ $0.03 \mathrm{ml}$ and $1.13 \pm 0.06 \mathrm{ml}$, respectively, revealing a whole-body glycocalyx exclusion volume for circulating plasma of $0.41 \pm 0.04 \mathrm{ml}(18.2 \pm 1.9 \mathrm{ml} / \mathrm{kg}$ body weight $)$. This volume was less $(0.23 \pm 0.05 \mathrm{ml})$ in mice that had received an intravenous bolus of hyaluronidase (35 U). Determination of circulating plasma volume using large size dextrans $(70,500,2000 \mathrm{kDa})$ revealed plasma volumes that were larger $(\mathrm{P}<0.05)$ than that derived from the dilution of labeled red blood cells and hematocrit, and as a result estimated exclusion of plasma by the glycocalyx was smaller for these dextrans. Our data indicate that the whole-body blood-excluding glycocalyx volume measurement using dilution of labeled red blood cells and large vessel hematocrit is susceptible to enzymatic glycocalyx provocation. In contrast, presumed plasma tracers, such as large molecular weight dextrans appear to distribute in a significant part of the glycocalyx under resting conditions already, which results in an underestimation of endothelial glycocalyx volume.

\section{Introduction}

The endothelial glycocalyx forms a highly hydrated mesh of polysaccharide structures and adsorbed plasma proteins on the luminal side of all blood vessels [114,162]. It forms the true interface between the flowing blood and the endothelium and has been indicated to play an important role in homeostasis of the vascular wall [151]. Thus, enzymatic degradation of the glycocalyx was shown to be associated with impairment of shear mediated NO production [135], increased adhesion of leukocytes and 
Acute hyaluronidase treatment reduced systemic glycocalyx volume in mice

platelets [21,92], and vascular barrier loss $[54,142,150]$. Because the glycocalyx has been shown to be vulnerable to perturbation by hyperglycemic [104,172], hyperlipidemic [156], and inflammatory conditions [48,93,116], measurement of glycocalyx properties might provide information on the state of vascular protection in an individual.

Nieuwdorp and coworkers recently estimated whole-body glycocalyx volumes in human subjects by determining the difference between circulating plasma volume, derived from the dilution of labeled red blood cells (RBCs) and large vessel hematocrit, and the distribution volume of $40 \mathrm{kDa}$ dextrans [103,104]. In this way, they obtained significant blood-excluding glycocalyx volumes of about $1.7 \mathrm{~L}$ in healthy subjects, which were decreased to $\sim 0.5 \mathrm{~L}$ during 6 h hyperglycemia in the same subjects [104]. The methodology applied in these human studies is based on intravital microscopy studies of cremaster muscle microcirculation in rodents, which demonstrated that the glycocalyx under resting conditions excludes both RBCs and flowing plasma, while $40 \mathrm{kDa}$ dextrans seem to have unimpaired access to the entire intravascular volume, including the glycocalyx $[47,121,125,129,152,157]$. It has so far, however, not been tested whether the systemic glycocalyx volume measurement is sensitive to a provoked specific challenge of the glycocalyx.

Alternative to the determination of circulating plasma volume using labeled RBCs and hematocrit, as done in the studies of Nieuwdorp et al. [103, 104], circulating plasma volume has commonly been estimated using the dilution of presumed plasma tracers. It was recognized in these studies that the measured plasma volumes typically exceed the calculated plasma volume based on the dilution of RBCs [55], and it was suggested that this disparity was caused by the fact that the used plasma tracers do not only distribute into the circulating part of the plasma but enter the glycocalyx domain as well [55]. Indeed, previous experimental studies in hamster [12] and mouse [150,172], which measured whole-body glycocalyx exclusion volume using large molecular weight (MW) dextrans or albumin as plasma tracer, showed that these tracers typically distributed in a volume that was modestly smaller than the volume for $40 \mathrm{kDa}$ dextrans. As a result, the estimated whole-body glycocalyx exclusion volumes in these studies were smaller than the aforementioned estimations in humans [103,104].

In the current study, we aimed at validating the systemic glycocalyx volume measurement, as used in our previous human studies [103,104], by measuring the effect of a 
bolus of hyaluronidase on this parameter in a mouse model. Hyaluronidase targets hyaluronan glycosaminoglycans within the glycocalyx and has previously been shown to degrade the glycocalyx-associated polysaccharide mesh in isolated rat hearts [142] and to impair glycocalyx exclusion of fluorescently labeled macromolecules in hamster cremaster muscle capillaries [47]. Further, we evaluated whether large MW dextrans of 70,500 , and $2000 \mathrm{kDa}$, which have been considered to be typical plasma tracers, also distribute in a volume comprising the glycocalyx, by comparing their distribution volume to the circulating plasma volume derived from the dilution of labeled RBCs and large vessel hematocrit, and the distribution volume of $40 \mathrm{kDa}$ dextrans.

\section{Methods}

\section{General surgery and anesthesia}

All procedures and protocols were approved by the Animal Care and Use committee of the Academic Medical Center in Amsterdam, the Netherlands. Experiments were performed on $\mathrm{C} 57 \mathrm{Bl} / 6$ mice of both sexes $\left(\mathrm{o}^{7}: \mathrm{N}=16\right.$, body weight $(\mathrm{BW}) 25.1$ $\pm 1.0 \mathrm{~g}$; \%: $\mathrm{N}=20$, BW $23.8 \pm 0.7 \mathrm{~g}$; Charles River Europe), that received standard chow and water ad libitum until the day before the experiment. Mice were overnight fasted (16-20 h) and were anesthetized with an intraperitoneal (ip) injection of ketamine hydrochloride $(125 \mathrm{mg} / \mathrm{kg})$, medetomidine $(0.2 \mathrm{mg} / \mathrm{kg})$, and atropine $(0.5 \mathrm{mg} / \mathrm{kg})[150,172]$. Tracheotomy was performed and mechanical ventilation was started by connecting the trachea tube to a pressure-controlled ventilator (SAR-830/P; CWE). Animals were ventilated with a gas mixture of $1: 1 \mathrm{O}_{2}: \mathrm{N}_{2}$. Respiration rate was set at 90 breaths/min with a peak inspiratory pressure of $18 \mathrm{cmH}_{2} \mathrm{O}$ and a positive end-expiratory pressure of $2 \mathrm{cmH}_{2} \mathrm{O}$. Anesthesia was maintained by continuous ip infusion at a rate of $10 \mathrm{ml} / \mathrm{kg} / \mathrm{h}$ of ketamine $(3.5 \mathrm{mg} / \mathrm{ml})$, medetomidine $(20 \mu \mathrm{g} / \mathrm{ml})$, and atropine $(7.5 \mu \mathrm{g} / \mathrm{ml})$. The right carotid artery and jugular vein were cannulated for monitoring systemic blood pressure and heart rate, and administration of hyaluronidase/saline and fluorescent tracers, respectively. Depth of anesthesia was checked according to stability of blood pressure and lack of toe pinch reflex, and infusion rate of anesthetics was adjusted if necessary. Esophageal temperature was maintained at $\sim 37^{\circ} \mathrm{C}$ by placement of the animal on a temperature-controlled heating 
Acute hyaluronidase treatment reduced systemic glycocalyx volume in mice

pad and radiant heat. At the end of the experimental procedures, the mouse was given an overdose of ketamine.

\section{Tracer infusion and blood collection}

Whole-body glycocalyx excluded volume was calculated from the difference in distribution volume of a plasma tracer of choice versus that of simultaneously infused Texas Red-labeled 40 kDa dextrans (Dex-40; Invitrogen-Molecular Probes) in the mouse circulation [150,172]. The following plasma tracers were used: 1) circulating plasma as derived from the dilution of labeled red blood cells and hematocrit, and 2) FITClabeled dextrans of $70 \mathrm{kDa}$ (Dex-70), $500 \mathrm{kDa}$ (Dex-500), or $2000 \mathrm{kDa}$ (Dex-2000) (Sigma-Aldrich).

\section{Red blood cells}

Circulating plasma was determined from the dilution of fluorescein-labeled RBCs, revealing circulating red blood cell volume, and large vessel hematocrit [103, 104]. Blood ( 1 ml) was collected from a donor mouse by cardiac punction, centrifuged, and the red blood cells labeled with sodium fluorescein $(250 \mathrm{mg} / \mathrm{ml})$ for $5 \mathrm{~min}$. After washing, the labeled cells were resuspended in saline to the initial volume. Two min before infusion, $0.1 \mathrm{ml}$ of the labeled blood was mixed with an equal volume of Dex$40(15 \mathrm{mg} / \mathrm{ml})$ and $0.1 \mathrm{ml}$ of this tracer mix was infused in the animal in $1 \mathrm{~min}$. Blood samples $(5 \mu \mathrm{l})$ were collected in heparinized capillaries through tail bleeding at $\mathrm{t}=-5$ (pre), 2, 3, 4, and 5 min after start of the infusion for determination of the fraction of labeled red blood cells $[103,104]$. In addition, $30 \mu \mathrm{l}$ samples were collected in $\sim 30 \mathrm{~s}$ at $\mathrm{t}=-5$ (pre), 2, 5, 10, 15, 20, and $30 \mathrm{~min}$ for determination of Dex-40 concentrations $[150,172]$.

\section{Dextrans}

$0.1 \mathrm{ml}$ of dextran mix $(2.5 \mathrm{mg} / \mathrm{ml}$ FITC-labeled dextran $+10 \mathrm{mg} / \mathrm{ml} \mathrm{Dex}-40$ in phosphate-buffered saline) was manually infused in the jugular vein in $1 \mathrm{~min}$, and blood was subsequently sampled $(30 \mu \mathrm{l})$ through tail bleeding at $\mathrm{t}=-5$ (pre), 2, 5, $10,15,20$, and 30 min after start of the tracer infusion [150,172]. In about $60 \%$ of the animals, visibly produced urine was gathered during the experiment in a capillary 
tube, and the remaining bladder content collected after the experiment (at $\mathrm{t}=35$ $\min )[172]$.

\section{Tracer analysis}

\section{Red blood cells}

Labeled RBCs were measured using a FACScan analyzer (FACSCalibur; Becton Dickinson, Mountain View, CA), with at least 100,000 cells being counted to measure the circulating fraction of labeled cells [103,104]. Data were analyzed using Cellquest (Becton Dickinson, San Jose, CA). The circulating plasma volume was calculated as

$$
V_{\text {plasma }}=\frac{\left[(1-H c t) \times V_{r b c}\right]}{H c t}
$$

where $\mathrm{V}_{r b c}$ is the circulating red blood cell volume ([1/circulating fraction of labeled RBCs $] \times$ volume of labeled cells injected) and Hct is the hematocrit $[103,104]$. The fraction of labeled cells at $\mathrm{t}=2,3,4,5$ min was averaged and used as circulating fraction; unlabeled erythrocytes obtained before the injection ( $t=-5$ min) served as negative controls.

\section{Dextrans}

Capillaries were centrifuged, hematocrit was determined, and plasma collected and stored at $-20^{\circ} \mathrm{C}$ until fluorescence analysis. In each sample, fluorescence was measured at 490/535 nm (excitation/emission) for the FITC-labeled dextrans (Dex-70, Dex500, Dex-2000) and at 595/615 nm for Dex-40 with a spectrophotometer (VICTOR; PerkinElmer) and dextran concentrations calculated in reference to defined dilutions of the infused tracer mix in plasma from donor mice $[150,172]$. Plasma dextran concentrations were normalized to the amount injected, and the concentration-time curve of an individual tracer was fitted with a mono-exponential function [104,150,172]. The initial distribution volume of each dextran was determined from the extrapolated dilution at the start of tracer injection. Vascular clearance was defined as the percentage decrease in tracer concentration at the end of the measurements $(\mathrm{t}=35 \mathrm{~min})$ compared to the extrapolated concentration at the start of tracer injection ( $t=0 \mathrm{~min}$ ) [150]. In a similar fashion as the plasma samples, dextran concentrations were determined and 
Acute hyaluronidase treatment reduced systemic glycocalyx volume in mice

normalized to the amount injected in the urine samples, stored at $-20^{\circ} \mathrm{C}$ until fluorescence analysis. The recovery of dextrans in the urine in an experiment (as percentage of amount injected) was determined from the urine dextran concentration and total volume of urine sampled.

\section{Experimental protocols}

The following two protocols were explored (see table 4.1).

protocol 1: Evaluation of the effect of hyaluronidase on whole-body glycocalyx exclusion volume

After instrumentation and a 15 min equilibration period, female mice were given a bolus ( $0.05 \mathrm{ml}$ in $1 \mathrm{~min}$ through jugular vein) of saline alone (control group) or hyaluronidase (35 U, Type IV-S; Sigma-Aldrich) in saline. Next, after a $60 \mathrm{~min}$ incubation period, a bolus injection of mixed labeled RBCs/Dex-40 or Dex-500/Dex40 was administered, respectively (see table 4.1), and blood samples were collected at the aforementioned time-points. The choice for a 60 min incubation period was based on reported observations that glycocalyx exclusion was maximally impaired 45 min to 1 hour after administration of the enzyme [12,47].

\section{protocol 2: Assessment of glycocalyx exclusion of large MW dextrans versus that of circulating plasma derived from $\mathrm{RBC}$ dilution}

In male mice, distribution volumes of Dex-70, Dex-2000, and circulating plasma were each compared to that of simultaneously infused Dex-40, and the resulting glycocalyx exclusion volumes of these tracers were calculated (see table 4.1).

\section{Data analysis}

Effects of hyaluronidase (protocol 1) were compared to control (saline) mice using ttests. Differences in tracer behavior (protocol 2) were analyzed using one-way analysis of variance, assuming glycocalyx exclusion to increase with tracer size (Dex-40< Dex-70 $<$ Dex-500 < Dex-2000 < circulating plasma). Post-hoc comparisons were performed using Tukey tests. Results were considered statistically significant with 


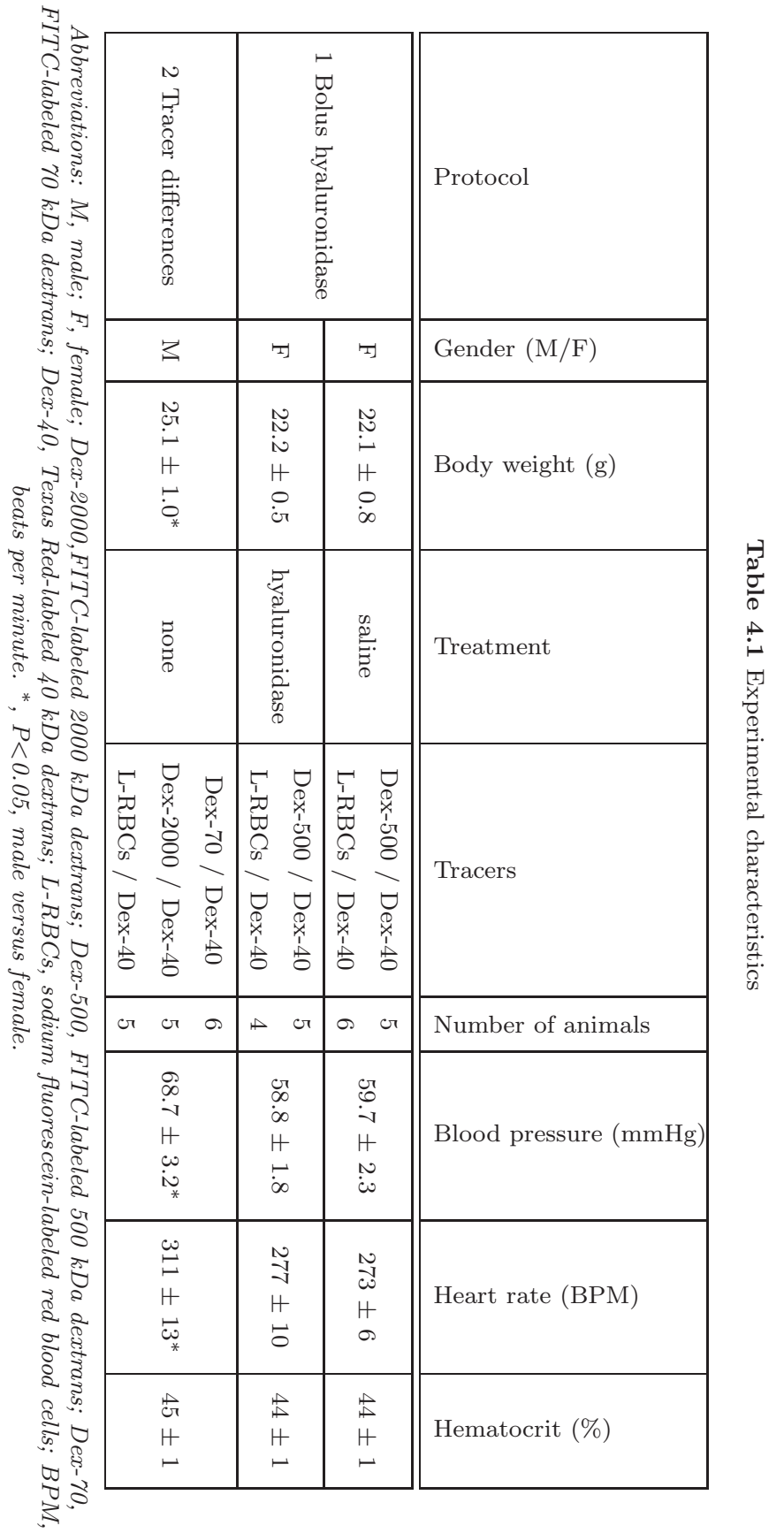


$\mathrm{P} \leq 0.05$. Summary data are reported as means $\pm \mathrm{SEM}$, with $\mathrm{N}$ referring to the number of animals studied.

\section{Results}

Hemodynamic data are shown in table 4.1. Blood pressure and heart rate are low compared to values measured by our group in awake animals. The reduction in blood pressure and heart rate is inherent to the type of anesthetics used in these experiments. Comparing the two protocols, blood pressure and heart rate were lower in the female mice (protocol 1) compared to the male mice (protocol 2). There was no effect of hyaluronidase on blood pressure, heart rate, and hematocrit (protocol 1).

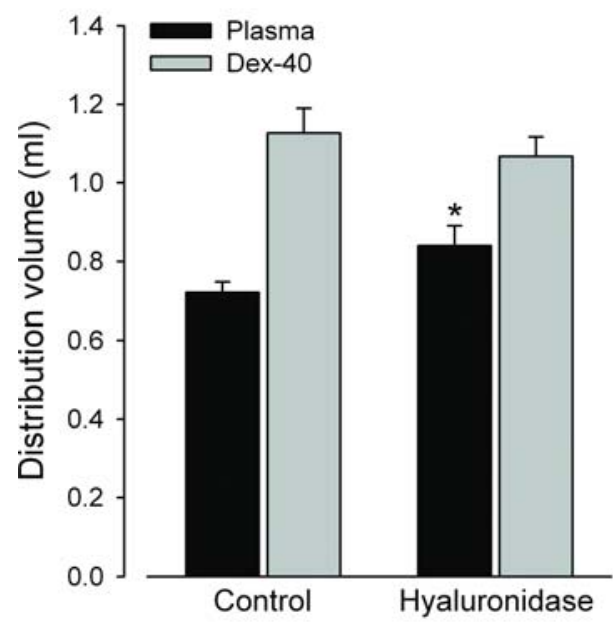

(a)

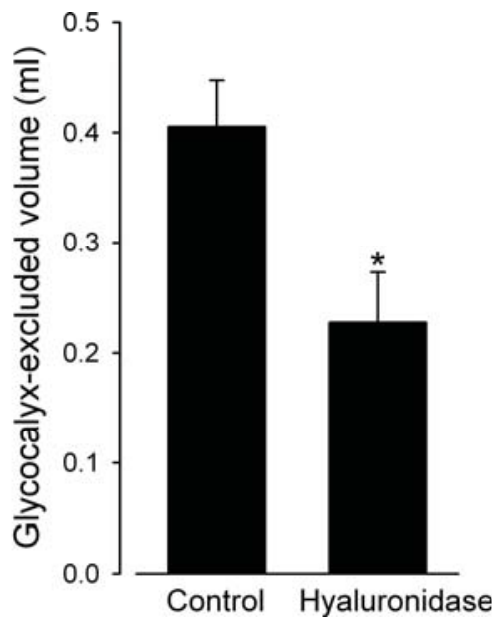

(b)

Figure 4.1 Hyaluronidase reduces whole-body glycocalyx exclusion of circulating plasma. Data points represent average \pm SEM. (a) Volume of distribution of circulating plasma, derived from dilution of labeled red blood cells and hematocrit, and simultaneously infused Dex-40, derived from extrapolated concentration at $\mathrm{t}=0 \mathrm{~min}$, in control (saline) and hyaluronidase treated female mice. * $\mathrm{P}=0.05$ versus control. (b) Plasma-excluding glycocalyx volume, calculated from the difference between distribution volume of Dex-40 and plasma. ${ }^{*} \mathrm{P}<0.05$ versus control. 


\section{Protocol 1: Effect of hyaluronidase on whole-body glycocalyx exclusion volume}

In the control group of female mice, circulating plasma volume, calculated from fluorescein-labeled RBC distribution and hematocrit, was $0.72 \pm 0.03 \mathrm{ml}$, which when corrected for body weight corresponds with a volume of $32.2 \pm 1.5 \mathrm{ml} / \mathrm{kg} \mathrm{BW}$ (figure 4.1a).

Dex-40 volume was larger $(\mathrm{P}<0.001)$ than plasma volume, $1.13 \pm 0.06 \mathrm{ml}$, resulting in a plasma-excluding glycocalyx volume of $0.41 \pm 0.04 \mathrm{ml}(18.2 \pm 1.9 \mathrm{ml} / \mathrm{kg} \mathrm{BW}$, figure $4.1 \mathrm{~b})$. Plasma volume was increased $(\mathrm{P}=0.05)$ in the hyaluronidase treated mice without a change in Dex-40 volume and resulted in a smaller $(\mathrm{P}<0.05)$ glycocalyx volume of $0.23 \pm 0.05 \mathrm{ml}$ (figure 4.1).



(a)

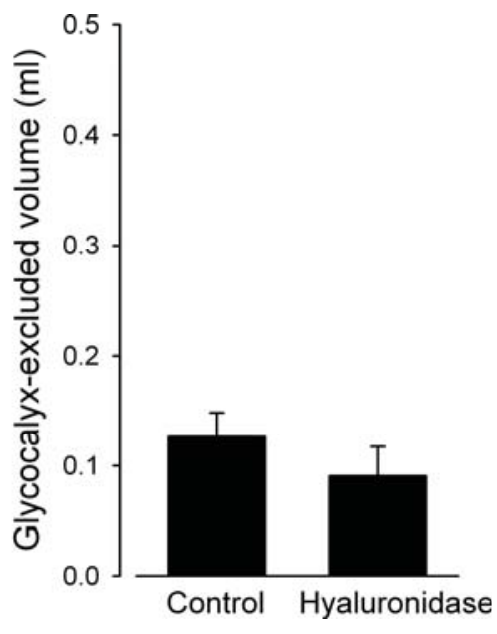

(b)

Figure 4.2 Hyaluronidase does not significantly affect glycocalyx exclusion of Dex-500. Data points represent average \pm SEM. (a) Volume of distribution of Dex-500 and Dex-40, derived from extrapolated concentration at $\mathrm{t}=0 \mathrm{~min}$, in control (saline) and hyaluronidase treated female mice. (b) Dex-500 excluding glycocalyx volume, calculated from the difference between distribution volume of Dex-40 and Dex-500.

Dex-500 distribution volume in control female mice, inferred from the extrapolated plasma concentration at the start of tracer infusion $(\mathrm{t}=0 \mathrm{~min})$, was $0.86 \pm 0.09 \mathrm{ml}$ 
Acute hyaluronidase treatment reduced systemic glycocalyx volume in mice

and smaller than $(\mathrm{P}<0.005)$ the distribution volume of simultaneously injected Dex40 (figure 4.2a). The difference between the two volumes, reflecting the Dex-500 excluding glycocalyx volume, was $0.13 \pm 0.02 \mathrm{ml}$, which is smaller $(\mathrm{P}<0.05)$ than the plasma-excluding glycocalyx volume calculated from the difference between distribution volume of Dex-40 and plasma. Treatment of mice with hyaluronidase did not change significantly distribution volumes of Dex-500 and Dex-40, and as a result, the Dex-500 excluding glycocalyx volume was not affected by hyaluronidase treatment (figure 4.2b). Dex-500 was slowly cleared from the circulation in control mice during the experiment $(5.6 \pm 1.8 \% / 35 \mathrm{~min})$, and in line with our previous study, in which clearance of Dex-70 increased many-fold after the administration of a bolus of hyaluronidase [150], we observed an increased $(\mathrm{P}=0.01)$ clearance of Dex-500 one hour after the bolus infusion of hyaluronidase (22 $\pm 6 \%$ / 35 min). In contrast to Dex-500, Dex-40 was already rapidly lost from the circulation in control mice, as reflected by a clearance of $55 \pm 1 \%$ after $35 \mathrm{~min}$, and was comparable in hyaluronidase treated mice $(58 \pm 5 \% / 35 \mathrm{~min})$.

\section{Protocol 2: Glycocalyx exclusion of large MW dextrans versus circulating plasma}

Circulating plasma volume in male mice was $0.67 \pm 0.04 \mathrm{ml}$ (figure 4.3) and, together with a measured Dex-40 volume of $1.19 \pm 0.05 \mathrm{ml}$, the plasma-excluding glycocalyx volume was $0.51 \pm 0.05 \mathrm{ml}(19.8 \pm 2.1 \mathrm{ml} / \mathrm{kg} \mathrm{BW})$ (figure 4.4). There were no gender differences with respect to circulating plasma volume and Dex-40 volume (protocol 1), and consequently no gender difference in systemic glycocalyx exclusion volume for circulating plasma.

Comparison of distribution volumes of circulating plasma with the large MW dextrans of 70 and $2000 \mathrm{kDa}$, and simultaneously infused smaller MW Dex-40, revealed an increase $(\mathrm{P}<0.001)$ in volume from plasma $<$ Dex-2000 $<$ Dex-70 $<$ Dex-40 (figure 4.3). Post-hoc comparison revealed differences in distribution volume between circulating plasma and Dex-70 $(\mathrm{P}<0.05)$, between circulating plasma and Dex-40 $(\mathrm{P}<0.001)$, and between Dex-2000 and Dex-40 ( $\mathrm{P}<0.05)$. Glycocalyx exclusion volume, i.e., the difference between distribution volume of Dex-40 and plasma tracer, increased $(\mathrm{P}<0.001)$ from Dex-70 < Dex-2000 < plasma (figure 4.4). This effect was explained by an 


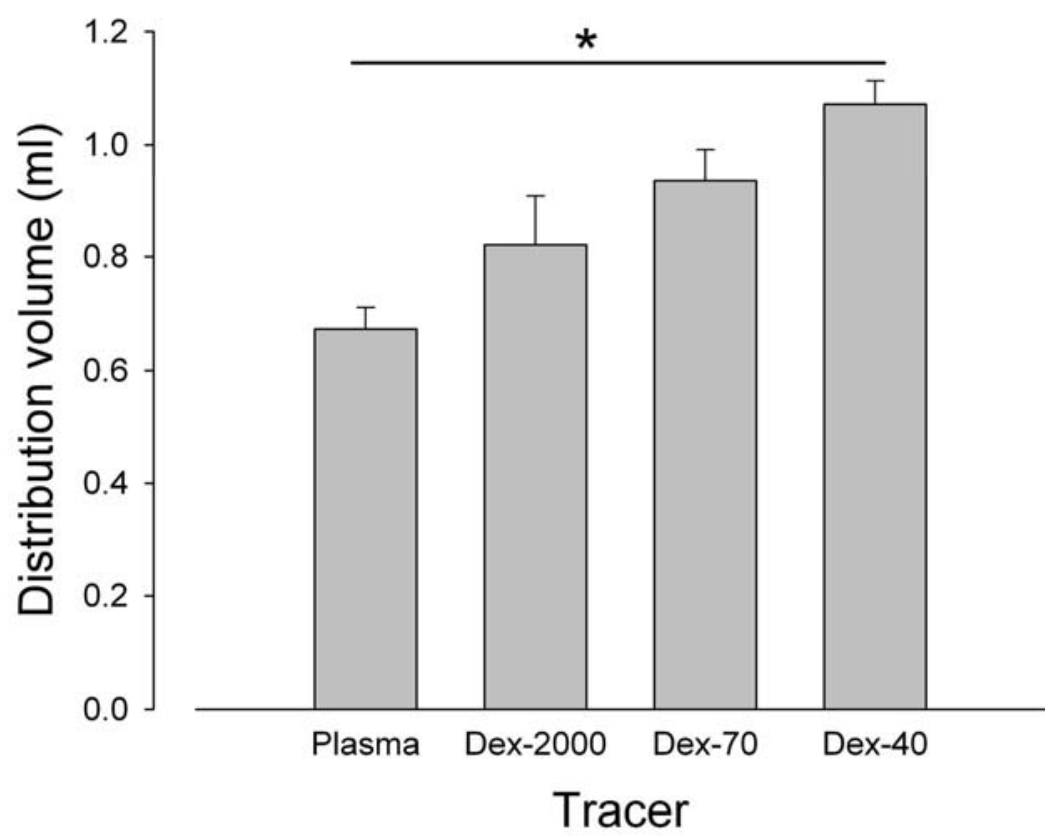

Figure 4.3 Volumes of distribution of circulating plasma, derived from dilution of labeled red blood cells and hematocrit, Dex-2000, Dex-70, and Dex-40 in male mice. In each experiment, a plasma tracer was co-infused with Dex-40; Dex-40 data were pooled across experiments. Data points represent average \pm SEM. ${ }^{*} \mathrm{P}<0.001$, volume of distribution increased with decreasing tracer size (plasma $<$ Dex-2000 $<$ Dex-70 $<$ Dex-40).

increased glycocalyx exclusion of circulating plasma compared to the Dex-2000 and Dex-70 dextrans (both $\mathrm{P}<0.001$ ).

Systemic vascular clearance was $16.8 \pm 2.5 \%$ / 35 min for Dex-2000, $13.9 \pm 6.5 \%$ / 35 min for Dex-70, and $52.1 \pm 1.9 \%$ / 35 min for Dex-40 $(\mathrm{P}<0.001$ compared to the other dextrans). The recovery of dextrans in the urine (at $\mathrm{t}=35 \mathrm{~min}$, as $\%$ of amount injected) was also higher for Dex-40 compared to the other dextrans (both $\mathrm{P}<0.001$ ); recovery was $30.6 \pm 3.1 \%$ / $35 \mathrm{~min}$ for Dex-40, $6.2 \pm 1.2 \%$ / $35 \mathrm{~min}$ for Dex-70, and $0.6 \pm 0.2 \% / 35 \mathrm{~min}$ for Dex-2000. Not all of the dextrans cleared from the vasculature were found in the urine of mice, this hold in particular true for Dex-2000. Gonzalez-Castillo and coworkers [37] showed by using dextranase that dextrans can bind to luminal endothelial lectinic structures. We expect, therefore, that the dex- 


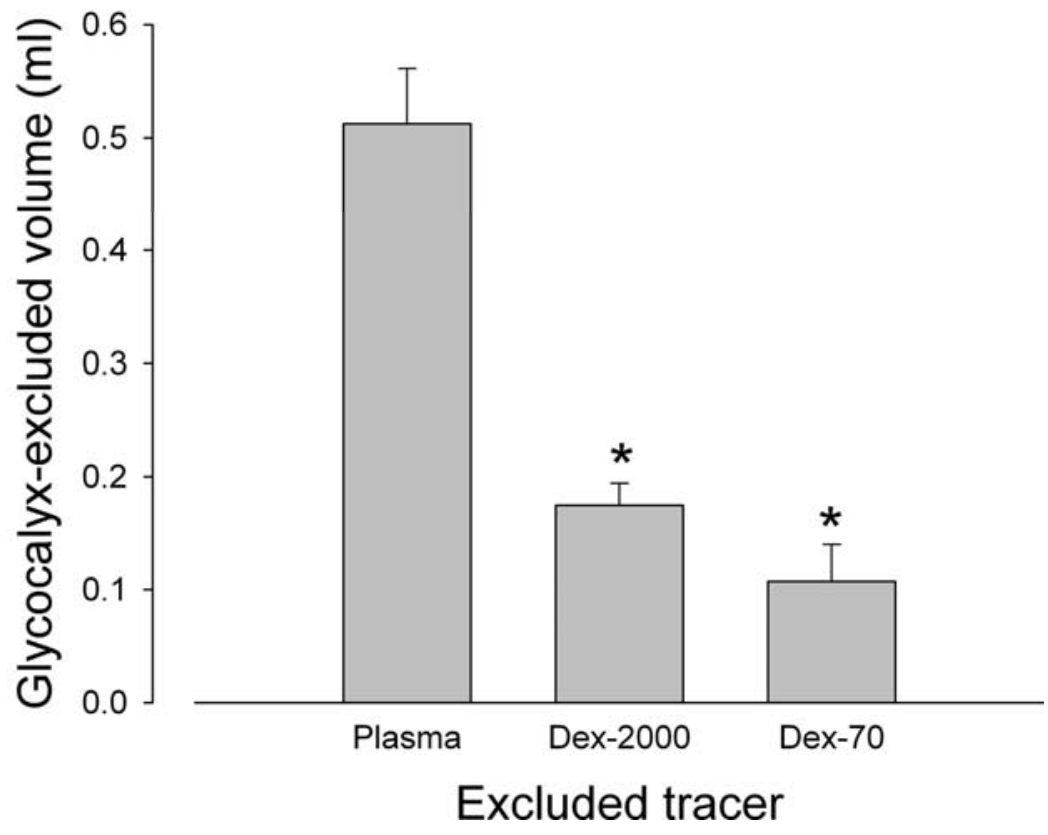

Figure 4.4 Glycocalyx-excluded volumes for circulating plasma, Dex-2000, and Dex-70, calculated from the difference between distribution volume of Dex-40 and respective plasma tracer (see figure 4.3). * $\mathrm{P}<0.001$ compared to plasma-excluding glycocalyx volume.

trans not measured in the plasma or found in the urine are bound to the endothelium. Figure 4.5 illustrates the difference in renal clearance between Dex-40 versus the large MW dextrans. In this figure, the amount of dextran collected in urine (as\% of the amount injected) until $35 \mathrm{~min}$ after injection is plotted against the amount of tracer cleared from the circulation after $35 \mathrm{~min}$ for all experiments in the current and our previous [150] study, in which urine was collected ( $60 \%$ of the mice); data for the FITC-labeled 70, 500, and $2000 \mathrm{kDa}$ were pooled (Dex-Large). On average, the ratio of the amount of Dex-40 recovered in urine to its clearance was $0.55 \pm 0.06$, illustrating that $55 \%$ of the Dex-40 lost from the circulation after its injection appeared in the urine. This ratio was $0.18 \pm 0.07$ for the pooled large MW dextrans $(\mathrm{P}<0.001$ compared to Dex-40). The ratio's were not different in the hyaluronidase treated animals. 


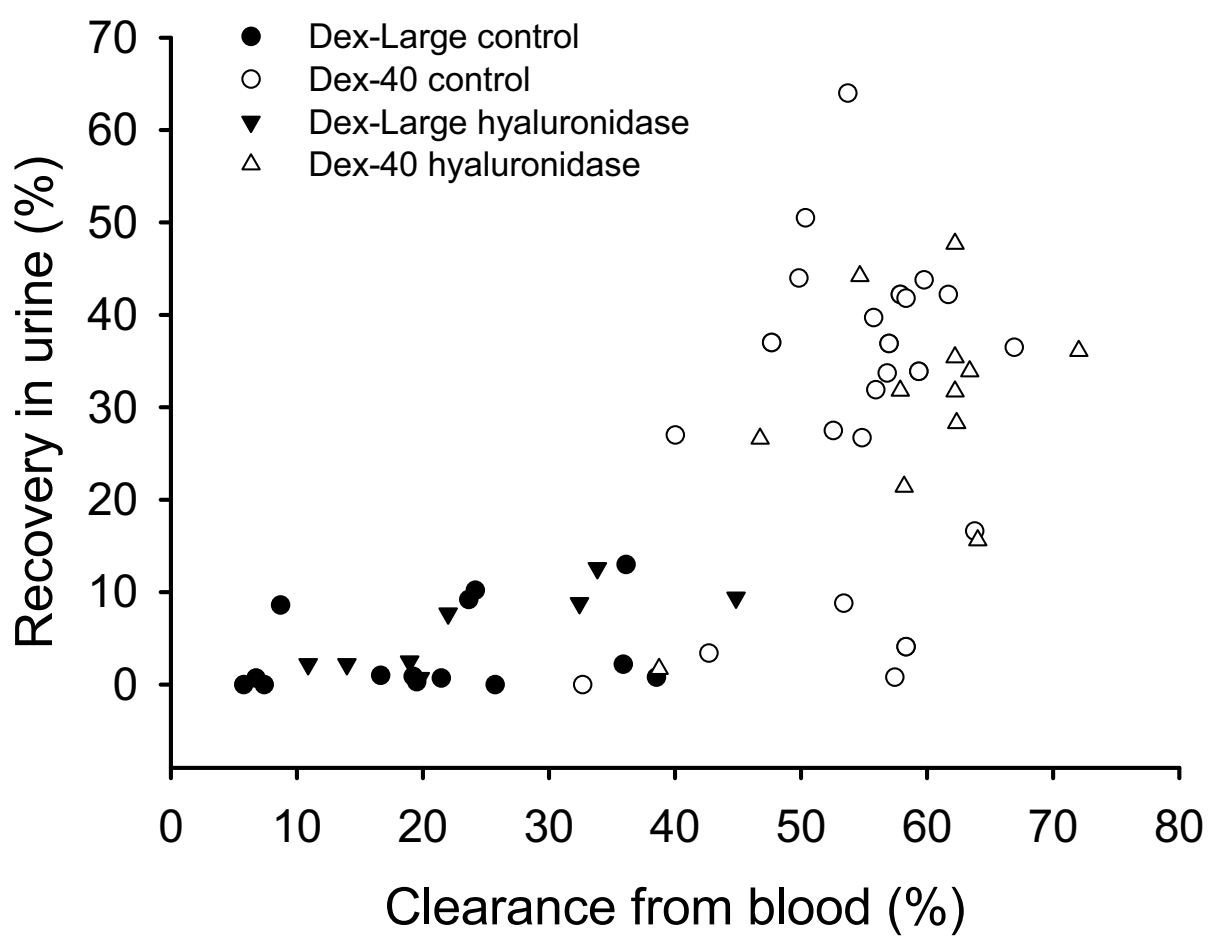

Figure 4.5 Differences in systemic and renal clearance between large MW dextrans and Dex- 40 . X-axis, calculated clearance of tracer (Dex-Large, large MW dextrans (FITC-labeled 70, 500, and $2000 \mathrm{kDa}$; pooled); Dex-40, Texas Red-labeled $40 \mathrm{kDa}$ dextrans) from the vasculature at $35 \mathrm{~min}$ after injection; Y-axis, dextran collected in urine at $35 \mathrm{~min}$ after injection. Data points represent experiments of current study and a previous study [150], in which urine was collected.

\section{Discussion}

Recent estimations of whole-body glycocalyx volume using labeled RBCs as plasma tracer suggested the presence of a substantial volume of blood-excluding glycocalyx in the human circulation $[103,104]$. In the current study, we used the same methodology in anesthetized $\mathrm{C} 57 \mathrm{Bl} / 6$ mice and found glycocalyx exclusion for circulating plasma (female mice: $18.2 \mathrm{ml} / \mathrm{kg} \mathrm{BW}$; male mice: $19.8 \mathrm{ml} / \mathrm{kg} \mathrm{BW}$ ) to be comparable to that found in the human studies $(1.7 \mathrm{~L}$ in a $75 \mathrm{~kg}$ subject $\sim 23 \mathrm{ml} / \mathrm{kg} \mathrm{BW})$. Single intravenous administration of hyaluronidase resulted in a $\sim 45 \%$ reduction in whole-body 
Acute hyaluronidase treatment reduced systemic glycocalyx volume in mice

plasma-excluding glycocalyx volume. In contrast, hyaluronidase treatment did not significantly reduce the exclusion volume of $500 \mathrm{kDa}$ dextrans, which, as 70 and 2000 $\mathrm{kDa}$ dextrans, were found to distribute in a significant part of the blood-excluding glycocalyx volume under control conditions already. Our data indicate that the wholebody blood-excluding glycocalyx volume measurement using dilution of labeled RBCs and large vessel hematocrit is sensitive to enzymatic glycocalyx provocation. Furthermore, the data suggest that presumed plasma tracers, i.e., large MW dextrans in the current study, are typically not excluded to the same extent as circulating plasma, and, as a result, evaluation of exclusion of these tracers might be less valuable for assessment of glycocalyx perturbation.

\section{Tracer dilution: methodological considerations}

We determined systemic glycocalyx exclusion properties of different plasma tracers from the difference in initial distribution volume of the tracer of choice versus that of Dex-40, using the tracer dilution technique. This methodology has recently been applied by us $[103,150,172]$ and others [12], and is based on intravital microscopic observations of intravascular tracer distribution in cremaster capillaries $[47,152,158]$ and in isolated mesenteric arteries [144]. These observations revealed the glycocalyx to exclude RBCs and to constitute a barrier for FITC-labeled dextrans of $70 \mathrm{kDa}$ and larger, without apparent hindrance of Texas Red-labeled $40 \mathrm{kDa}$ dextrans. Tracer dilution in the current experiments was performed by intravenous (jugular vein) infusion of the tracer followed by measurement of the concentration in the plasma after presumed complete mixture of the tracer in the vascular system. The fraction of labeled RBCs was constant for the FACS samples taken at $\mathrm{t}=2,3,4$, and 5 min after tracer injection (data not shown), suggesting mixing to be complete at our first sampling point, $\mathrm{t}=2 \mathrm{~min}$. For the dextrans, subsequent to $\mathrm{t}=2$, samples were taken at $\mathrm{t}=5,10,15,20$, and $30 \mathrm{~min}$ to enable adequate appraisal of the dextran clearance kinetics $[150,172]$. Systemic clearance was considerable for the Dex-40, around 50$60 \%$ in $35 \mathrm{~min}$, and more than half of this appeared in the urine in this time period, illustrating that elimination of this tracer by the kidneys occurs rapidly. Vascular clearance was much smaller for the FITC-labeled dextrans of $70 \mathrm{kDa}$ and larger $(13.9$ $\pm 6.5 \%$ / 35 min for Dex-70, and $16.8 \pm 2.5 \%$ / 35 min for Dex-2000), and may represent slow equilibration of the tracer with the glycocalyx domain [158] rather than 
renal clearance, since recovery of these dextrans in the urine was very low (figure 4.5). Altogether, these data exemplify the permselective barrier properties of the vascular system, including the glomerular barrier, in anesthetized mice. Dynamic information on renal clearance of the various tracers was, however, not obtained in the current experiments since the measured tracer recovery in the urine reflected the integrated recovery for $35 \mathrm{~min}$ after tracer infusion.

\section{Plasma tracer distribution differences}

Comparison of the distribution volumes of circulating plasma versus Dex-40 (figures 4.1 and 4.4) in the current study revealed a very comparable relative amount of bloodexcluding glycocalyx in the vasculature of the mouse compared to that reported in our human studies [104] (figure 4.1: $0.41 \mathrm{ml}$ glycocalyx in $1.13 \mathrm{ml} \mathrm{Dex}-40=36 \%$ in mice; 1.7 L glycocalyx in $4.7 \mathrm{~L}$ Dex-40 $=36 \%$ in humans). In microvessels with an average anatomic diameter of $5 \mu \mathrm{m}$, this degree of "glycocalyx filling" would be explained by a thickness of the glycocalyx of $1 \mu \mathrm{m}$. Similar glycocalyx dimensions were estimated by Pries and coworkers for explaining the discrepancy between experimental estimates of apparent viscosity or flow resistance in microvessels in vitro versus in vivo $[113,115]$. Distribution volumes of 70,500 , and $2000 \mathrm{kDa}$ dextrans were considerably larger than that for circulating plasma derived from the dilution of labeled RBCs and hematocrit, and this disparity caused the volume of glycocalyx exclusion for the dextrans to be only $25-35 \%$ of that for plasma. In agreement with the lesser exclusion of FITClabeled large dextrans are the reported exclusion zones of only $0.4-0.5 \mu \mathrm{m}$ for these dextrans in cremaster capillaries with intravital microscopy [47, 152,158].

Our findings support clinical observations showing that tracer-based estimations of plasma volume generally reveal greater volumes of distribution than those based on the dilution of labeled RBCs and large vessel hematocrit $[55,86]$. The difference in estimated plasma volume between the two approaches has been handled in the past by use of the so-called F-cell ratio, which is defined as the ratio between the whole-body hematocrit, derived from red blood cell and total blood volume (i.e., tracer-estimated plasma volume + red blood cell volume), and large vessel hematocrit. Estimation of this F-cell ratio from the average distribution volumes in the current study reveals a ratio of about 0.85 for the FITC-labeled large MW dextrans in our mice, which is somewhat higher than the ratio of 0.74 reported by Gunji et al. (2002) in anesthetized 
Acute hyaluronidase treatment reduced systemic glycocalyx volume in mice

C57Bl/6 mice using radioactive albumin as plasma tracer [39]. We propose that this difference reflects the greater tendency for albumin to permeate into the glycocalyx compared to the FITC-dextrans $[55,158]$. While in the current study even the largest $(2000 \mathrm{kDa})$ dextrans were still found to partly enter the glycocalyx domain, labeled hydroxyethyl starch was reported in humans to give a close to equivalent estimation of the plasma volume compared to the volume derived from dilution of labeled RBCs and hematocrit [82]. We hypothesize that species differences as well as differences in molecular configuration and size between hydroxyethyl starch versus dextrans might be the underlying cause. In addition, comparison of our measurements with those in humans may be confounded by the fact that that the current measurements were performed during daytime, which is the inactive period for mice, and under conditions of anesthesia [55]. In this respect, Cabrales and coworkers reported relative (to Dex40 volume) glycocalyx exclusion volumes for Dex-70 and Dex-2000 in awake hamsters that were about two-fold greater than the current observations in anesthetized mice [12].

\section{Sensitivity to glycocalyx provocation}

Hyaluronidase infusion at a dose that has been used in the past for targeting of the glycocalyx $[12,47,150]$ decreased plasma-excluding glycocalyx volume with $\sim 45 \%$, illustrating that glycocalyx measurements using red blood cells and large vessel hematocrit are capable to detect imposed changes in glycocalyx volume. Exclusion volume for Dex-500, which was in control animals already smaller than the plasma-excluding glycocalyx volume in hyaluronidase treated mice, was not significantly affected by the enzymatic treatment, agreeing with intravital microscopic observations showing limited effect of hyaluronidase on 580 and $2000 \mathrm{kDa}$ dextran permeation into the glycocalyx domain of cremaster capillaries [47]. In contrast to the unaltered exclusion at $\mathrm{t}=0$ min, vascular clearance of Dex-500 was increased after hyaluronidase treatment in the current study, similar to our previous findings in mice of an increased clearance of Dex-70, but unaltered initial Dex-70 exclusion, after hyaluronidase treatment [150]. Our data, therefore, indicate that evaluation of exclusion properties of plasma tracers, which already distribute in a substantial part of the glycocalyx under control conditions, might be too insensitive to monitor glycocalyx perturbation. Nevertheless, an increased clearance of these tracers seems to represent enhanced movement of the 
tracer across the glycocalyx domain, and also other barriers, because of impairment of its exclusion properties. This understanding might explain the apparent discrepancy between our observations and the reported decreases in exclusion volumes of Dex-70 and albumin after hyaluronidase treatment in hamsters [12]. In their experiments, tracer concentration was determined by linear regression of measured concentrations at only $\mathrm{t}=2$ and $5 \mathrm{~min}$ after tracer infusion, leaving the possibility that the reported decrease in exclusion volume for Dex-70 and albumin after hyaluronidase reflected an increased clearance of these tracers rather than a true decrease in their initial exclusion [12].

\section{Conclusion}

Given the important role of the glycocalyx in vascular homeostasis, measurement of glycocalyx properties might provide information on the state of vascular protection in an individual. In the current study in anesthetized mice, we found that bloodexcluding whole-body glycocalyx volume using dilution of labeled RBCs and hematocrit versus dilution of $40 \mathrm{kDa}$ dextrans decreased by $\sim 45 \%$ after enzymatic glycocalyx provocation using a bolus of hyaluronidase, illustrating that this measurement is sensitive to glycocalyx perturbation. In contrast, hyaluronidase treatment did not affect the exclusion volume of $500 \mathrm{kDa}$ dextrans, and this could be explained by the fact that large MW dextrans distributed in a significant part of the plasma-excluding glycocalyx volume under control conditions already. These findings emphasize the importance of the right choice of tracer for evaluation of whole-body glycocalyx volume. 


\section{Whole-body recruitment of glycocalyx volume during}

intravenous adenosine infusion in goats

The contents of this chapter are based on Judith Brands, Hans Vink and Jurgen WGE Van Teeffelen, Whole-body recruitment of glycocalyx volume during intravenous adenosine infusion in goats, in preparation 


\section{Abstract}

Introduction Adenosine has been suggested to recruit vascular volume in heart and muscle by increasing the accessibility of the glycocalyx for flowing plasma, in addition to vasodilation of the resistance vessels. We hypothesized that systemic intravenous administration of adenosine increases blood accessibility of the intravascular glycocalyx on a whole-body level. Methods In anesthetized goats $(\mathrm{N}=6)$ systemic blood-excluded glycocalyx volume was measured by comparing the intravascular distribution volume of the glycocalyx accessible tracer dextrans with a molecular weight of $40 \mathrm{kDa}$ (Dex-40) to that of circulating plasma, derived from the dilution of labeled red blood cells and large vessel hematocrit. Systemic glycocalyx volume was determined at baseline and during intravenous infusion of adenosine (157 \pm 11.6 $\mu \mathrm{g} / \mathrm{kg} / \mathrm{min})$. Results Blood inaccessible glycocalyx volume decreased from $488.3 \pm$ 98.1 to $62.2 \pm 62.8 \mathrm{ml}(\mathrm{P}<0.01)$ during adenosine administration. The decrease in blood excluding glycocalyx volume was not associated with an increase in circulating plasma volume (from $625.8 \pm 49.4$ to $734.0 \pm 46.3 \mathrm{ml}$, N.S.) but associated with a significant decrease in Dex-40 distribution volume (from $1114.0 \pm 71.1$ to $796.2 \pm$ $58.1 \mathrm{ml}, \mathrm{P}<0.01)$. Conclusion Intravenous administration of adenosine results in a decrease in blood inaccessible glycocalyx volume at whole-body level. The simultaneous observed decrease in Dex-40 distribution volume suggests that the reduction in glycocalyx volume coincides with a reduction in accessible vascular volume, e.g. due to loss of number or volume of perfused capillaries.

\section{Introduction}

Maximal coronary hyperemia, required for the measurement of fractional flow reserve and coronary flow reserve, is clinically achieved by administration of the vasodilator adenosine, either by continuous intravenous infusion or as an intracoronary bolus $[15,57,164]$. Recent studies indicate that during adenosine, besides vasodilation of the resistance vessels, also the accessibility of the glycocalyx for plasma and macromolecules increases $[29,74,110,154]$. In dog hearts, the adenosine-mediated increase in coronary conductance was observed to exceed maximum conductance during coronary reactive hyperemia [154]. Enzymatic degradation of the glycocalyx, however, 
Adenosine decreases systemic glycocalyx volume

revealed equal conductances during reactive hyperemia and adenosine-induced hyperemia, suggesting that adenosine-induced coronary hyperemia may include recruitment of glycocalyx volume. The data from that study correspond with earlier intravital microscopic data in cremaster muscle showing a reduction in exclusion of large dextrans from the glycocalyx and concomitant increase in capillary tube hematocrit during adenosine $[29,74,110]$. Similar effects on glycocalyx exclusion properties and capillary tube hematocrit were recently demonstrated for bradykinin and SNP [152]. These observations indicate that vasodilators may increase microvascular blood content not only by vasodilation of resistance vessels, but also by increasing blood accessibility of the glycocalyx. The effect of adenosine on whole-body blood excluding glycocalyx volume has not been studied yet. The glycocalyx volume in the body has been estimated to be $20-25 \mathrm{ml} / \mathrm{kg}$ body weight $[103,104]$ and plain recruitment of this volume for blood perfusion during adenosine, as has been suggested in the cremaster muscle of hamsters $[29,110]$, would confront the cardiovascular system with a severe filling problem. For that reason, we hypothesize that during intravenous adenosine administration glycocalyx volume might be recruited, but to limit the fall in peripheral vascular resistance, a decrease in anatomic vascular volume might be expected, similar as has been reported during provoked loss of glycocalyx volume in humans $[101,103,104]$. In the current study, we therefore investigated the effect of intravenous adenosine infusion on whole-body blood exclusion by the glycocalyx. In anesthetized goats, systemic blood-excluded glycocalyx volume was determined from the difference in distribution volume of circulating plasma, derived from the dilution of labeled red blood cells and large vessel hematocrit, and the distribution volume of a tracer for both plasma and glycocalyx volume, dextrans with a molecular weight of $40 \mathrm{kDa}$ (Dex-40) [103,104]. Tracers were infused during control conditions and during intravenous infusion of a dose of adenosine ( $\sim 160 \mu \mathrm{g} / \mathrm{kg} / \mathrm{min})$ which is clinically used to evoke maximal coronary hyperemia. To obtain the distribution volumes of the tracers in the circulation, systemic blood samples were drawn at regular time points for 12 minutes after tracer infusion. In addition, systemic blood pressure, heart rate, and coronary blood flow were measured as well. 


\section{Materials and methods}

\section{Animal preparation}

All of the procedures and protocols were approved by the Animal Care and Use committee of the Academic Medical Center. Studies were conducted in accordance with the National Institutes of Health Guide for the Care and Use of Laboratory Animals. Experiments were performed on adult female goats of $17-29 \mathrm{~kg}(\mathrm{~N}=6)$. At the beginning of an experiment, the goats were anesthetized with an intramuscular injection of Nimatek (15 mg/kg, Eurovet Animal Health BV) and Dormicum $(0.75 \mathrm{mg} / \mathrm{kg}$, Roche). Goats were intubated and ventilated with a 1:2 $\mathrm{O}_{2}$ :air mixture. Anesthesia was maintained by intravenous administration of Sufenta $(9.375 \mu \mathrm{g} / \mathrm{kg} / \mathrm{h}$, JanssenCilag), Dormicum (0.625 mg/kg/h, Roche) and Propofol (10 mg/kg/h, B.Braun). Depth of anesthesia was adjusted according to stability of femoral artery blood pressure $\left(\mathrm{P}_{f e m}\right)$ and heart rate $(\mathrm{HR})$. Arterial and coronary venous blood gases, hematocrit and pH were measured every 30 minutes and analyzed using a Radiometer ABL 510 (Radiometer). When necessary, ventilation was adjusted to maintain oxygen and $\mathrm{CO}_{2}$ pressures within physiological limits, and sodium bicarbonate administered to avoid acidosis.

\section{Surgery}

The following surgical procedures were performed. First, catheters were placed in the femoral vein, for the infusion of tracers, and via the left carotid artery in the aorta, for arterial blood sampling. Next, a left thoracotomy was performed in the fourth intercostal space and one of the ribs was removed. The great cardiac vein was cannulated via the azygos vein to obtain coronary venous blood samples. A flowprobe (3mm Transonic flowprobe, Transonic Systems Inc) was placed around one of the major coronary branches (LAD or LCX) to measure coronary blood flow $\left(\mathrm{Q}_{\text {cor }}\right)$. The $\mathrm{P}_{\text {fem }}, \mathrm{Q}_{\text {cor }}$ and $\mathrm{HR}$ (determined from $\mathrm{P}_{\text {fem }}$ ) were stored for off-line analysis (100 Hz PowerLab Data Acquisition Systems, ADInstruments). At the end of the experimental procedures a battery was placed on the heart to induce ventricular fibrillation. 


\section{Experimental protocol}

After surgery, the preparation was allowed to equilibrate for 30 minutes. Systemic glycocalyx volume and circulating blood volume were measured first at baseline and subsequently during 20 minutes of intravenous infusion of $157 \pm 11.6 \mu \mathrm{g} / \mathrm{kg} / \mathrm{min}$ adenosine $[15,67,108,134,164]$ (range $120-180 \mu \mathrm{g} / \mathrm{kg} / \mathrm{min}$ ). Adenosine administration was limited to 20 minutes in total to resemble a clinical dose as close as possible yet long enough to collect a sufficient amount of samples for the measurement of Dex40 and blood distribution volume. A five minute delay time was allowed between the start of adenosine administration and the measurement of systemic distribution volumes. Within this time period a new steady state for coronary blood flow, heart rate and blood pressure was obtained.

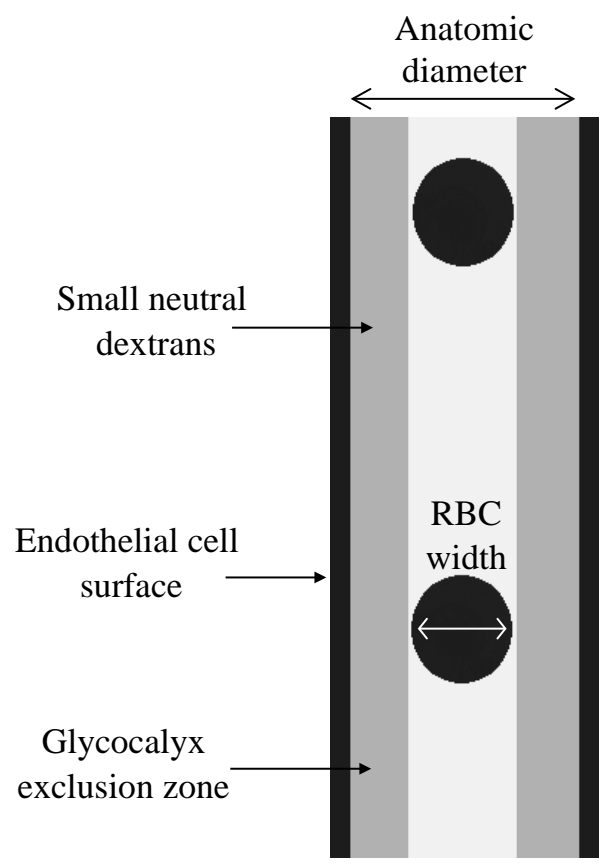

Figure 5.1 Schematic overview of distribution of RBCs and small dextrans (from intravital microscopy studies in cremaster) $[157,158]$. RBCs are excluded from the glycocalyx, while Dex-40 has unimpaired access to the glycocalyx. 


\section{Tracers}

Systemic glycocalyx volume was determined from the difference in distribution volume of circulating plasma (derived from red blood cell volume and large vessel hematocrit) and Dex-40 (100 mg/ml Rheomacrodex; NPBI International) [103,104], see figure 5.1. At the start of the surgery, $40 \mathrm{ml}$ of blood was taken per measurement and centrifuged at $1200 \mathrm{~g}$ for 5 minutes. Subsequently, the centrifuged red blood cells were mixed with sodium fluorescein $(250 \mathrm{mg} / \mathrm{ml})$ for $10 \mathrm{~min}$. After being washed, the labeled red blood cells were resuspended in Dex-40 (75-150 ml).

\section{Injection}

Previous to each measurement blood was collected (pre-sample), after which the tracers were administered in the femoral vein with a syringe pump $(30 \mathrm{ml} / \mathrm{min}$, B.Braun). Before the first injection of the tracers, a single bolus of $5 \mathrm{ml}$ dextrans with a molecular weight of $1 \mathrm{kDa}$ (Promiten, NPBI) was injected to attenuate the risk for anaphylactic reactions. The tracers were infused within 15 minutes after the administration of Promiten [80]. In the initial two experiments, $150 \mathrm{ml}$ of Dex-40 was used (15 gr) to be certain to have sufficiently high concentrations in the blood for detection. In these experiments, infusion of the tracers was done in $\sim 5$ minutes. In the other experiments, the volume of Dex-40 was reduced to $75 \mathrm{ml}(7.5 \mathrm{gr})$, resulting in a reduced infusion time ( $\sim 3$ minutes) and dextran concentrations that were still higher than the detection limit.

\section{Sampling}

Blood was sampled from both the great cardiac vein and aorta at $t=3,5,8$ and 12 minutes after the infusion of tracers was stopped. The first sample was taken after approximately 3 minutes to guarantee complete mixture of the tracers with the blood. To collect only blood samples during the administration of adenosine, the last sample, in contrast to the study in human subjects [103,104], was taken 12 minutes after the infusion of the tracers. 
Adenosine decreases systemic glycocalyx volume

\section{Data analysis}

Labeled red blood cell fraction was measured using a FACScan analyzer (FACSCalibur; Becton Dickinson). The fraction of labeled red blood cells was found to be constant between 3 and 12 minutes after the dextrans were infused, and the average value of the data within this period was taken during further analysis. The average fraction of labeled red blood cells versus the total red blood cell pool was used to estimate circulating red blood cell volume [106]. The circulating plasma volume $\left(\mathrm{V}_{\text {plasma }}\right)$ was calculated as:

$$
V_{\text {plasma }}=\frac{\left[\left(1-H_{\text {sys }}\right) \times V_{r b c}\right]}{H_{\text {sys }}}
$$

where $\mathrm{V}_{r b c}$ is the circulating red blood cell volume and $\mathrm{H}_{s y s}$ is the large vessel hematocrit, which was taken as the average of the great cardiac vein and aorta hematocrit. Total blood volume was defined as the sum of $\mathrm{V}_{\text {plasma }}$ and $\mathrm{V}_{r b c}$. After measuring the fraction of labeled red blood cells, blood was centrifuged and the plasma collected and stored at $-20^{\circ} \mathrm{C}$ until analyzed.

The Dex-40 concentration was calculated by measuring the increase in plasma glucose concentration in the post infusion samples after hydrolysis of the dextrans [146]. All measured glucose concentrations were corrected for the background glucose level $(0.7$ $\pm 0.04 \mathrm{mg} / \mathrm{ml}$ ) in the blood, measured in the pre-sample. The samples taken from the great cardiac vein and aorta were taken as duplicate measurements. To determine the initial Dex-40 distribution volume, the concentration of Dex-40 at $t_{\text {ini }}$ (which was halfway the infusion of the tracers, approximately 1.5 or 2.5 minutes after the start of the infusion of the tracers) was estimated by exponential fitting of the measured Dex-40 concentrations $[103,104]$, see figure 5.2. The distribution volume of Dex-40 was calculated by dividing the amount of dextran given (7500 or $15000 \mathrm{mg}$ ) by the background corrected concentration of dextrans at $t_{i n i}(\mathrm{mg} / \mathrm{ml})$. The clearance rate of the Dex-40 tracer was reflected by the power of the exponential fit [103].

All results are expressed as means \pm SEM. Differences in blood pressure, coronary blood flow and heart rate, as well as effects on volumes, hematocrit, initial Dex-40 concentrations and clearance rate were tested using a t-test. A probability value of $\mathrm{P}<0.05$ was considered significant. 

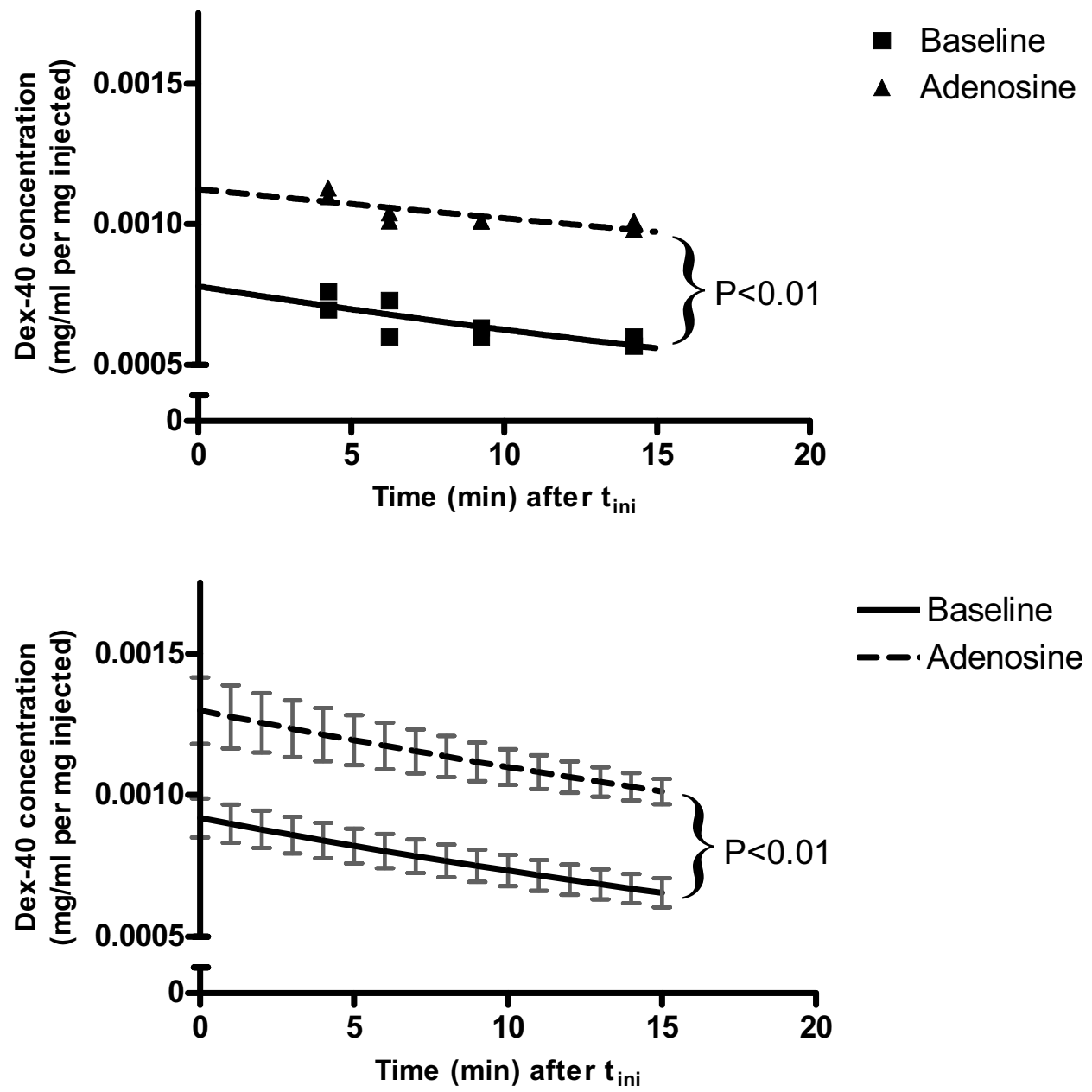

Figure 5.2 Top: A typical example of measured Dex-40 normalized to the amount of Dex-40 given $(\mathrm{mg} / \mathrm{ml}$ per $\mathrm{mg}$ injected tracer) over time and the exponential fit to determine the concentration at $\mathrm{t}_{\text {ini }}$ for baseline $(\boldsymbol{\square})$ and adenosine $(\boldsymbol{\Lambda})$. Bottom: Averaged plasma Dex-40 concentration curve normalized to the amount of Dex-40 given $(\mathrm{mg} / \mathrm{ml}$ per $\mathrm{mg}$ injected tracer) over time at baseline ( solid line, $\mathrm{y}=0.00092 \mathrm{e}^{-0.023 t}$ ) and during adenosine (dashed line, $\mathrm{y}=0.0013 \mathrm{e}^{-0.016 t}$ ). The averaged concentration curve is calculated using inter- and extrapolated data from the exponential models through the measured Dex-40 data (samples taken at $\mathrm{t}=3,5,8$ and 12 minutes used as input for the model). The standard error of the mean is indicated by the error bars. The clearance of Dex- 40 (power of the exponential fit) from the plasma was not statistically different in both measurements. Data are means \pm SEM. $\mathrm{P}<0.01$, difference in concentration of Dex-40 measured between adenosine and baseline measurements. 
Adenosine decreases systemic glycocalyx volume

\section{Results}

Baseline hemodynamic parameters are presented in table 5.1. Comparing baseline with adenosine measurements there was a $3.0 \pm 0.5$ fold increase in coronary flow $(\mathrm{P}<0.01)$, a significant increase in heart rate and a modest reduction in femoral artery blood pressure $(\mathrm{P}<0.08)$. There was also a significant decrease in hematocrit, see figure 5.3.

Table 5.1 Hemodynamic parameters at baseline and during adenosine

\begin{tabular}{|l|c|c|}
\hline & Baseline & Adenosine \\
\hline \hline $\mathrm{P}_{\text {fem }}(\mathrm{mmHg})$ & $91.0 \pm 7.8$ & $72.5 \pm 4.9$ \\
$\mathrm{HR}(\mathrm{beats} / \mathrm{min})$ & $117.7 \pm 7.9$ & $132.3 \pm 7.0^{*}$ \\
$\mathrm{Q}_{\text {cor }}(\mathrm{ml} / \mathrm{min})$ & $47.7 \pm 7.6$ & $133.6 \pm 19.8^{*}$ \\
\hline
\end{tabular}

Values are means $\pm S E M,{ }^{*}$ significant from baseline $(P<0.05)$.

The averaged extrapolated clearance curves of Dex-40 are depicted in figure 5.2 (bottom). At baseline the concentration of Dex-40 at $t_{i n i}$ was $9.2 \times 10^{-4} \pm 0.7 \times 10^{-4}$ $\mathrm{mg} / \mathrm{ml}$ per $\mathrm{mg}$ infused tracer. This concentration was significantly increased to $13.0 \times 10^{-4} \pm 1.2 \times 10^{-4} \mathrm{mg} / \mathrm{ml}$ per $\mathrm{mg}$ infused tracer during adenosine. Consistent with the diminished dilution of dextrans, the Dex-40 distribution volume decreased significantly from $1114.0 \pm 71.1 \mathrm{ml}$ at baseline to $796.2 \pm 58.1 \mathrm{ml}$ during adenosine $(\mathrm{P}<0.01)$. The clearance rate of the Dex-40 from the plasma was the same in both measurements, reflected by an unchanged exponential coefficient $(0.023 \pm 0.002$ $\min ^{-1}$ at baseline and $0.016 \pm 0.003 \mathrm{~min}^{-1}$ during adenosine, N.S.). The circulating red blood cell volume, plasma and total blood volume at baseline were not different from the volumes measured during adenosine administration; volumes are depicted in figure 5.3. The difference between the circulating plasma and Dex-40 distribution volume, i.e. the blood inaccessible glycocalyx volume, decreased significantly $(\mathrm{P}<0.01)$ comparing baseline with adenosine measurements (from $488.3 \pm 98.1 \mathrm{ml}$ to $62.2 \pm$ $62.8 \mathrm{ml}$, respectively), see figure 5.4 .

When comparing the different volumes given in figure 5.4, it can be seen that at baseline the blood inaccessible glycocalyx volume and the circulating plasma volume are nearly equal in size, while during adenosine infusion the distribution volume of Dex-40 closely corresponds to that of the circulating plasma volume. 


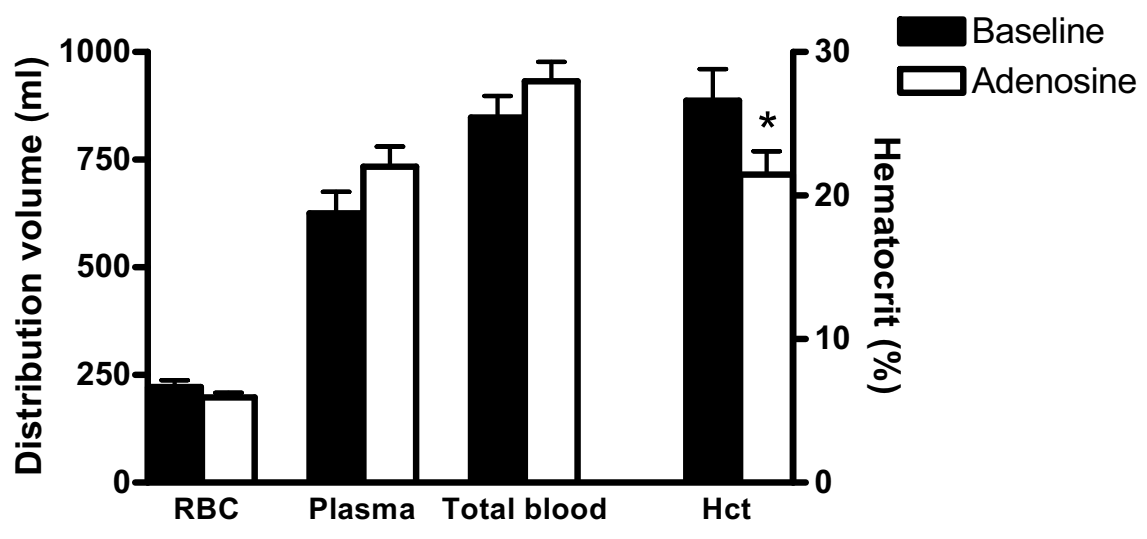

Figure 5.3 Red blood cell, plasma and total blood volume and average (aorta and coronary venous) hematocrit at baseline and during adenosine. Data are means $\pm \mathrm{SEM},{ }^{*} \mathrm{P}<0.05$, from baseline hematocrit measurements.

\section{Discussion}

In the present study in anesthetized goats, we found a $\sim 0.5 \mathrm{~L}$ difference in whole-body distribution volume between circulating blood and Dex-40 under control conditions, indicating a substantial exclusion of blood by the glycocalyx. During intravenous adenosine administration, this difference in distribution is almost completely lost, indicating decreased glycocalyx exclusion. We hypothesize that recruitment of glycocalyx volume during intravenous adenosine infusion promotes augmentation of microvascular volume and exchange capacity in heart and other tissues to which blood is redistributed during adenosine.

\section{Hemodynamics}

Adenosine is commonly used in the clinic since it is a potent vasodilator of the coronary bed. Indeed, we found a 3 -fold increase in coronary flow during intravenous infusion of a dose used in patients, in the face of a $\sim 20 \mathrm{mmHg}$ decrease in arterial blood pressure (table 5.1). A decrease in blood pressure occurred in all goats but was not significant 


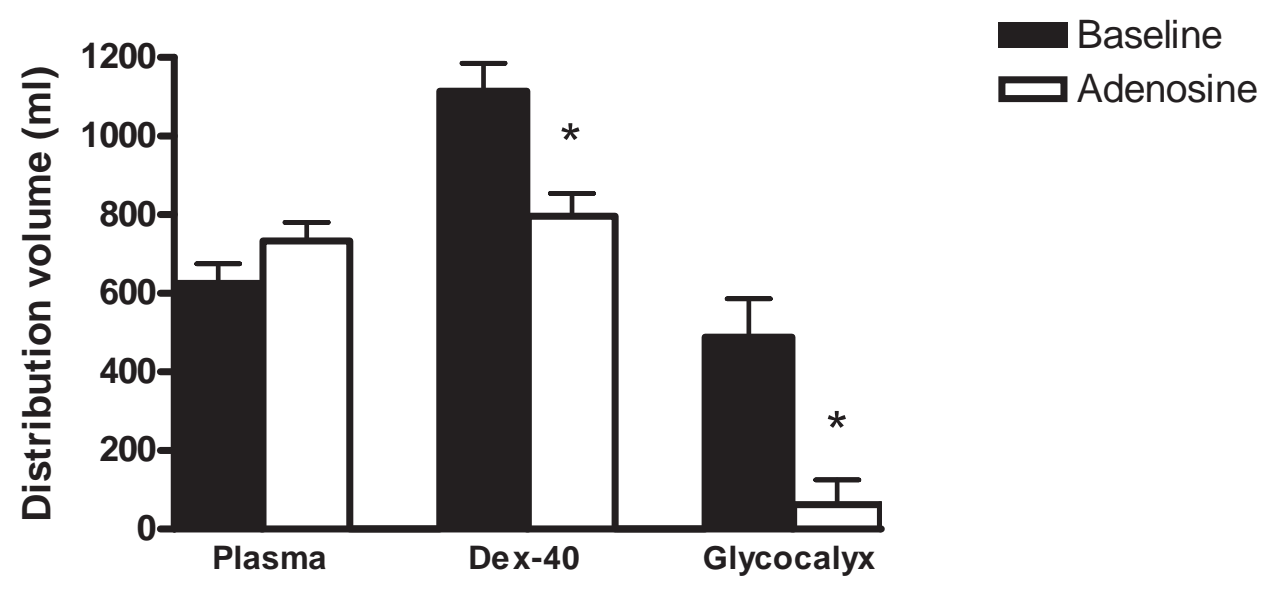

Figure 5.4 Plasma, Dex-40 and glycocalyx volume at baseline and during adenosine. Data are means \pm SEM, ${ }^{*} \mathrm{P}<0.05$, from baseline volume measurements.

$(\mathrm{P}<0.08)$, unlike the effects reported on anesthetized dogs $[23,105,120]$. Furthermore, we also observed a significant increase in heart rate. The effects of adenosine on heart rate in anesthetized animals is controversial $[23,105,120]$. Crystal et al. [23] suggested that the bradycardia observed in their study was due to direct suppression of pacemaker activity in the sinoatrial node by adenosine [56] that was adequate to override the baroreflex-mediated increase in heart rate associated with aortic hypotension. The effect of adenosine on the sinoatrial node appeared, however, to be dose dependent [56], just like the differences reported in literature. At a low dose, 0.53 $\mathrm{mg} / \mathrm{kg} / \mathrm{min}$, an increase in heart rate was reported [120], while at a 2.5-times higher dose the heart rate did not change significantly [105], whereas at a dose 4-times as high the heart rate was reduced [23]. Based on these results, we suggest that the dose used in our study, $157 \pm 11.6 \mu \mathrm{g} / \mathrm{kg} / \mathrm{min}$, enabled the baroreflex-mediated sympathetic increase to dictate the heart rate response.

Our hematocrit at baseline $(26.6 \pm 2.2 \%)$ is lower than the hematocrit measured in goats the day before an experiment $(32.6 \pm 1.6 \%$, see chapter 7$)$. The lower hematocrit is due to the sampling of $80 \mathrm{ml}$ of blood for the labeling of red blood cells that was compensated with the infusion of fluid, induction of anesthesia and surgery. Furthermore, average large vessel hematocrit (at baseline $26.4 \pm 2.3 \%$ for great cardiac 
vein and $26.9 \pm 2.2 \%$ for aorta) was significantly reduced during adenosine administration $(22.0 \pm 1.7 \%$ for great cardiac vein and $20.9 \pm 1.6 \%$ for aorta), see figure 5.3 . Most likely, hematocrit is decreased during the experiment because of dilution of the blood by the infusion of Dex-40 (75 or $150 \mathrm{ml}$ ) as well as Ringer (B.Braun) that was given as infusion fluid for the duration of the entire experiment.

\section{Effect of adenosine on endothelial glycocalyx}

The increase in coronary blood flow during adenosine has been contributed to a relaxation of predominantly the distal arterioles which have been shown to be most sensitive to adenosine [40,61]. In addition to resistance vessel relaxation, adenosine was indicated to increase perfused microvascular volume by modulation of the glycocalyx. First, Duling \& Klitzman [74] observed an increase in capillary tube hematocrit, using intravital microscopy, when cremaster muscle was superfused with adenosine. They suggested that the presence of a slow-moving plasma layer, representing the glycocalyx, contributed to the initial low hematocrit in capillaries. Later, Duling and coworkers showed that there was an apparent decrease in exclusion of large dextrans by the glycocalyx when adenosine was topically applied on the cremaster muscle $[29,110]$. More recently, an adenosine-mediated increase in coronary conductance was observed in dog hearts that exceeded maximum conductance during coronary reactive hyperemia, but only in the presence of an intact glycocalyx [154]. Degradation of the glycocalyx with the enzyme hyaluronidase revealed an equally increased conductance of reactive hyperemia and adenosine-induced hyperemia in the heart. In the current study, blood inaccessible glycocalyx volume was defined as the difference between the distribution volume of Dex-40 and circulating plasma volume determined using labeled red blood cells and large vessel hematocrit. The blood-excluding glycocalyx volume measured at baseline in this study, $23.8 \pm 5.3 \mathrm{ml} / \mathrm{kg}$ bodyweight, matches

nicely with the volumes found in humans, 20-25 ml/kg bodyweight [103, 104]. Furthermore, similar to the studies by Duling et al. in cremaster muscle, we observed a reduction in exclusion properties of the glycocalyx during adenosine administration. In the face of an unchanged circulating blood volume, the decrease in glycocalyx exclusion was associated with a decrease in Dex-40 distribution volume. Also in human subjects, Dex-40 volume was observed to be decreased upon perturbation of the glycocalyx by endotoxin infusion [101], 6 hour hyperglycemia [104], as well as in type 
Adenosine decreases systemic glycocalyx volume

I diabetics [103]. A reduction in Dex-40 distribution volume might be explained by a true decrease in vascular anatomic volume as well as a decrease in perfused (and hence tracer accessible) vascular volume. Both aspects have been demonstrated at the capillary level in rodents in response to provoked glycocalyx degradation. Thus, Van den Berg [142] showed that hyaluronan degradation in isolated rat hearts resulted in perivascular capillary edema formation which was associated with a decrease in anatomic diameter of the capillaries, while Cabrales [12] demonstrated a decrease in functional capillary density and increase in non-flowing capillaries in the hamster chamber window model after hyaluronidase treatment. We suggest that these reductions in microvascular blood volume will occur in organs were blood flow during adenosine is reduced, such as adipose tissue, kidney, liver and stomach [31,66], likely because of sympathetic nervous stimulated vasoconstriction of the resistance vessels. In contrast, blood flow towards the heart and skin and splanchnic blood flow is increased during adenosine $[31,66]$. We envision that in these tissues microvascular blood volume is increased as a result of both vasodilation of resistance vessels and recruitment of glycocalyx volume for perfusion.

\section{Methodological considerations}

The methodology used in the current study is based on the intravital microscopic observations that red blood cells and macromolecules are excluded from the glycocalyx while Dex-40 seems not to be hindered by it [158]. Dex-40 data was extrapolated to $\mathrm{t}_{\text {ini }}$, a point in time halfway the injection of the tracers. Extrapolation of the data to either start or end of infusion of the tracers would not have had an effect on the outcome of the study since baseline distribution volumes of $1063.9 \pm 70.2$ and 1166.9 $\pm 72.8 \mathrm{ml}$ for Dex-40 were calculated when extrapolating to the start and end of tracer infusion, respectively.

The whole-body measurement does not distinguish where the blood-excluding glycocalyx volume is residing in the circulation. The endothelial glycocalyx thickness seems to vary between different vessel types, and has been documented to range from $0.2-0.9 \mu \mathrm{m}$ in capillaries $[47,100,103,110,111,121,152,157,158]$, to $2-3 \mu \mathrm{m}$ in small arteries with a diameter of $\sim 150 \mu \mathrm{m}$ [144], to $4-5 \mu \mathrm{m}$ in carotid arteries [83]. These numbers indicate that during baseline conditions the glycocalyx occupies a large part of the anatomic vascular volume, particularly in the microcirculation. We hypothesize 
that in tissues with increased adenosine-induced blood flow, recruitment of glycocalyx volume causes a robust increase in vascular blood volume, especially capillary blood volume. The simultaneous dilation of resistance vessels (arterioles) primarily accounts for an increase in flow during adenosine infusion. As suggested before, we expect that in tissues with a reduction in flow during adenosine administration, the increase in microvascular volume due to glycocalyx recruitment is counteracted by loss of number of perfused capillaries and/or perivascular edema formation, resulting in a reduced blood perfused tissue volume.

\section{Conclusion}

In conclusion, we demonstrate in the current study in anesthetized goats that intravenous administration of a clinical dose of adenosine decreases blood excluded intravascular glycocalyx volume. The difference between the glycocalyx inaccessible and accessible tracer reduced to nearly zero. The decrease in blood inaccessible volume was associated with an almost equivalent decrease in perfused anatomic vascular volume. A decrease in blood excluding glycocalyx might provide a way by which adenosine can increase blood volume in tissues with increased adenosine-induced blood flow, such as the heart.

\section{Acknowledgments}

The authors would like to thank Carin Jansen and Kor Brandsma for their assistance. 


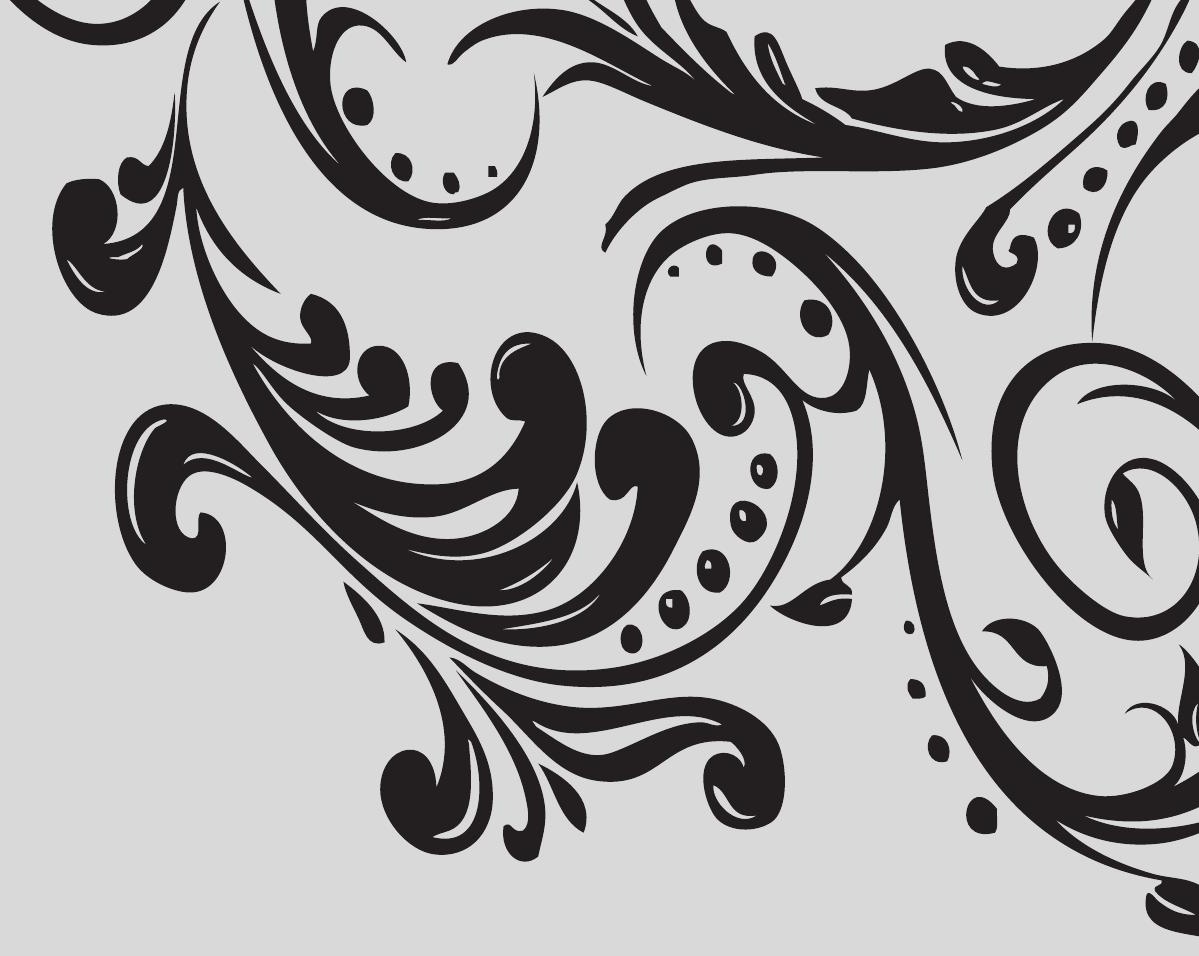

Coronary glycocalyx measurements 



\section{Comparison of four}

mathematical models for analysis of intravascular indicator dilution curves in the coronary circulation WGE Van Teeffelen, Comparison of four mathematical models for analysis of intravascular indicator dilution curves in the coronary circulation 


\section{Abstract}

Aim The indicator dilution technique has been used to estimate coronary blood and plasma volumes. In this study four models were compared to determine which model is best suited to measure coronary plasma volume using the indicator dilution technique; monoexponential and power-law model, which are models based on information from part of the descending slope, and gamma-variate and local density random walk (LDRW) model, which are based on data from the ascending slope, peak and part of descending slope of the curve. Method In anesthetized goats $(\mathrm{N}=14)$ with cannulated left main coronary artery and perfused using an extracorporeal perfusion system (delaying recirculation time with $>12$ seconds), the indicator dilution technique was applied to determine the distribution volume of a plasma tracer, high molecular weight (2000 kDa) dextrans. Coronary venous blood samples were taken every 0.5-1.0 sec using a roller pump and venous outflow curves analyzed using the four aforementioned models. Measurements were done at baseline (Base) and during an intracoronary administration of adenosine (Ado), which is hypothesized to increase coronary vascular volume. Results Significant differences were found between the different models with respect to coronary plasma volume at baseline and during adenosine; monoexponential $15.5 \pm 2.0$ (Base) and $25.2 \pm 3.0 \mathrm{ml} / 100 \mathrm{gr}$ (Ado), power-law $19.2 \pm 2.9$ (Base) and $31.0 \pm 3.8 \mathrm{ml} / 100 \mathrm{gr}$ (Ado), gamma-variate $13.9 \pm 1.7$ (Base) and $22.5 \pm 2.9 \mathrm{ml} / 100 \mathrm{gr}$ (Ado), and LDRW $14.2 \pm 1.8$ (Base) and $23.2 \pm 3.1 \mathrm{ml} / 100 \mathrm{gr}$ (Ado). Despite the differences in baseline volume between the models, all models appeared equally able to detect a similar relative increase in coronary vascular volume during adenosine. Although the gamma-variate and LDRW model did not describe the tail of the curve as good as the monoexponential or power-law model, these models seem preferable since all indicator dilution curves were fitted by them, they were less sensitive to noise and fewer data points along the descending slope of the curve were required for fitting the curves. Conclusion Coronary volume estimates derived by indicator dilution are influenced by the fitting procedure. Based on our results we suggest to use the gamma-variate or LDRW model for coronary blood volume measurements. 


\section{Introduction}

A large range of coronary vascular volumes has been reported in the literature (ranging from $4 \mathrm{ml} / 100 \mathrm{gr}$ to up to $17.8 \mathrm{ml} / 100 \mathrm{gr}[49,64,91,130,143,168])$. The differences in reported values can partly be explained by the fact that either only plasma volume or total blood volume were measured and by differences in experimental conditions (in vivo [22, 49, 91, 143, 168], beating isolated hearts [122] or arrested isolated hearts [63-65]), and the techniques used. Generally, approaches used can be divided into histological measurements, in which heart tissue is fixated and examinated using light or electron microscopy [35], the use of a silicone cast (usually in combination with histology) [63-65], and the dilution of specific blood or plasma indicators $[14,22,49,91,168]$.

The indicator dilution technique is a commonly used method in the clinic; it has been used in the cardiovascular field for many years to measure cardiac output [41], and blood flow and volumes [169] in the body. The theory and requirements of the indicator dilution technique have been reviewed by Meier et al. [84] and Zierler [169]. Indicator dilution is based on the principle that the flow or volume of a system can be measured by injection of a known quantity of an indicator into the system and measuring its concentration over time at a specific point (i.e., the indicator dilution curve). Using this curve, the mean transit time (MTT), which is the average time it takes for the indicator to travel from point of injection to point of sampling, can be derived. Multiplying the MTT with the indicator flow through the system, results in the distribution volume of the indicator, in our case the coronary vasculature.

Before the data of the indicator dilution curves can be used to derive the MTT, a model is commonly fitted through the data. One of the main reasons a model is used is to overcome contamination of the output signal by recirculating tracer [41]; rapidly circulating indicator passing the vascular volume of interest for the second time overlaps with slow moving indicator in the first pass. In literature many different models have been used to describe indicator dilution curves in the circulation, and four of the models that are most often used are the monoexponential, power-law, gamma-variate and local density random walk (LDRW) model [4, 7, 41, 42, 81,88]. With respect to the coronary circulation, the monoexponential fit has typically been used, and the following volumes have been reported: $9.4-14.8 \mathrm{ml} / 100 \mathrm{gr}$ tissue at perfusion flows 
of 40-220 $\mathrm{ml} / \mathrm{min} / 100 \mathrm{gr}$ in the study of Ziegler \& Goresky [168] and $15.6 \mathrm{ml} / 100 \mathrm{gr}$ in the studies of Hirsche \& Lochner [49] and Morgenstern et al. [91]. Surprisingly, the gamma-variate and LDRW fits, while proven to be good fits to describe indicator dilution curves for instance to determine cardiac output [88], have not been used for coronary volume measurements.

The aim of the current study was to determine which of the four mentioned models is best suitable (based on feasibility and signal-to-noise ratio) to fit tracer outflow curves when measuring coronary plasma volumes using the indicator dilution technique. Therefore, the indicator dilution technique was applied in vivo in open-chest anesthetized goats, in which the left main coronary artery was perfused via an extracorporeal perfusion system. This system enabled the injection of fluorescent $2000 \mathrm{kDa}$ dextrans as plasma tracer in the left main coronary artery at a controlled perfusion pressure and the online measurement of total left main coronary artery flow. The perfusion system had a relatively large volume $(\sim 110 \mathrm{ml})$ compared to the coronary system, resulting in a delayed recirculation since blood had first pass the entire perfusion system before it was pumped into the coronaries again. Blood was collected from the great cardiac vein with a roller pump and tracer outflow curves were constructed.

\section{Methods}

\section{General Surgery and Anesthesia}

All procedures and protocols were approved by the animal care and use committee of Maastricht University. The investigation conforms with the Guide for the Care and Use of Laboratory Animals. The data being used was from experiments on adult female goats of $18-28 \mathrm{~kg}(\mathrm{~N}=14)$ in which adenosine induced increases in coronary blood volume were measured to answer a different research question using this technique. In short, at the beginning of an experiment, the goats were anesthetized by an intramuscular injection of Nimatek $(15 \mathrm{mg} / \mathrm{kg}$, Eurovet Animal Health BV) and Dormicum (0.75 mg/kg, Roche), intubated and ventilated. Anesthesia was maintained by intravenous administration of Sufentanil-hameln $(9.375 \mu \mathrm{g} / \mathrm{kg} / \mathrm{h}$, Hameln Pharmaceuticals GmbH), Dormicum (0.625 mg/kg/h) and Propofol (10 mg/kg/h, Fresenius Kabi Nederland B.V.). Depth of anesthesia was adjusted according to stability 
of femoral artery blood pressure $\left(\mathrm{P}_{\text {fem }}\right)$ and heart rate $(\mathrm{HR})$.

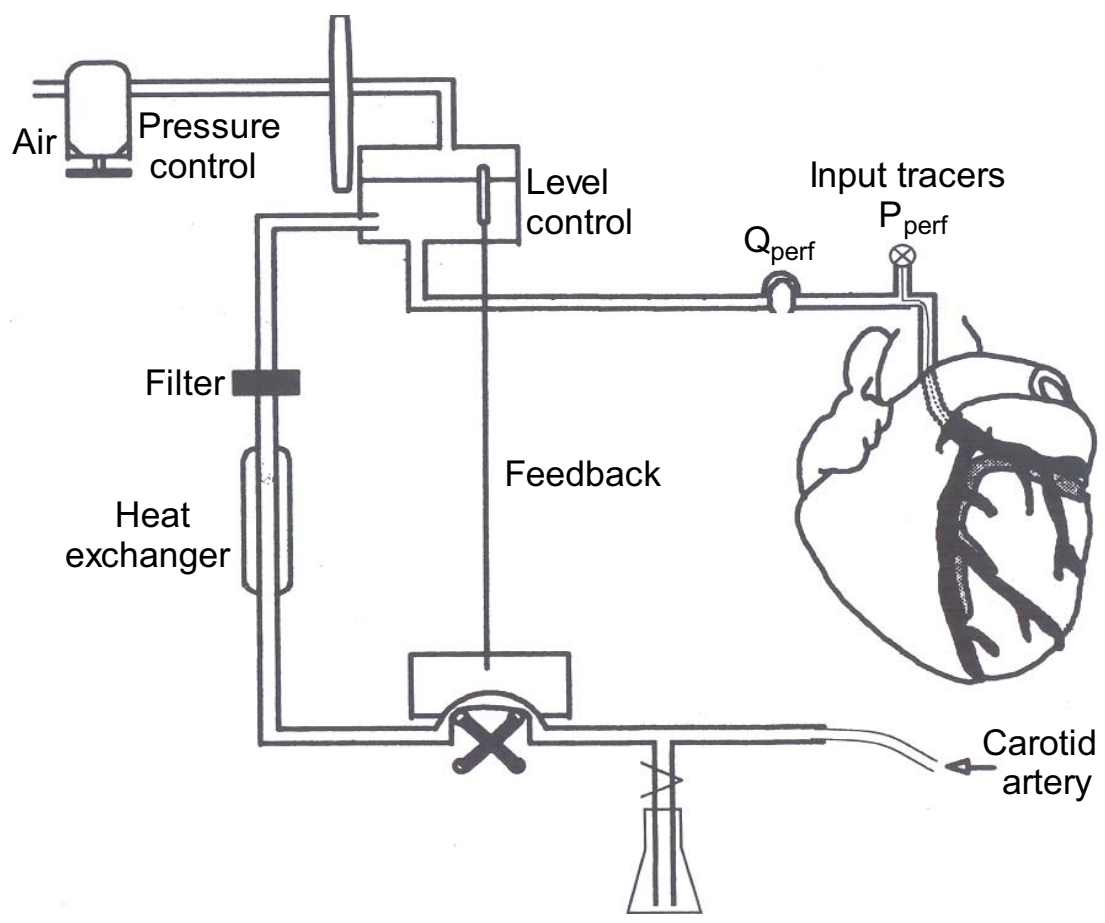

Figure 6.1 Arterial perfusion drainage system. Blood collected from the left carotid artery was heated and filtered, and circulated with a roller pump via a reservoir into the left main coronary artery. Blood level and pressure in the reservoir were kept constant. Perfusion pressure $\left(\mathrm{P}_{\text {perf } f}\right)$ was measured at the tip of the Gregg cannula. An inline flow probe was interpositioned into the perfusion system to measure coronary blood flow $\left(\mathrm{Q}_{\text {perf }}\right)$.

A left thoracotomy was performed in the fourth intercostal space and one of the ribs was removed. The great cardiac vein was cannulated via the azygos vein to obtain coronary venous blood samples. Subsequently, the left main coronary artery was dissected free and a ligature was placed around the vessel. A catheter (7F, Sentron) connected to a Sentron interface (Type. No 811-000) was inserted through the left auricle into the left ventricle to measure pressure $\left(\mathrm{P}_{l v}\right)$. Next, the left carotid artery was cannulated and a stainless steel Gregg cannula was inserted in the aorta via a purse string. Blood collected from the carotid artery was circulated through the perfusion system and back via the Gregg cannula into the aorta for 10 minutes. Hereafter, 
the Gregg cannula was ligated into the left main coronary artery, without disrupting the coronary flow. Coronary perfusion was controlled using a roller pump perfusion system (total volume $\sim 110 \mathrm{ml}$ ), see figure 6.1 [131]. The perfusion pressure $\left(\mathrm{P}_{\text {perf }}\right)$ was measured at the tip of the Gregg cannula and could be set at any arbitrary level (usually $120-130 \mathrm{mmHg}$ in control conditions). An inline flow probe (6 $\mathrm{mm}$ Transonic flowprobe, Transonic Systems Inc.) was interpositioned into the perfusion line of the perfusion system to measure coronary blood flow $\left(\mathrm{Q}_{\text {perf }}\right)$. The $\mathrm{P}_{\text {fem }}, \mathrm{P}_{\text {perf }}$, $\mathrm{P}_{l v}, \mathrm{Q}_{\text {perf }}$ and $\mathrm{HR}$ were stored on a personal computer for off-line analysis (IDEEQ $250 \mathrm{~Hz}$, IDEE). At the end of the experimental procedures a battery was placed on the heart to induce ventricular fibrillation. The heart was taken out and total heart weight as well as the weight of the perfused area was measured. Perfusion area was determined by injection of white paint into the left main coronary artery, and dissection and weighing of the demarcated area.

\section{Experimental protocol}

After surgery, the preparation was allowed to equilibrate for 30 minutes. In each animal the distribution volume of the plasma tracer FITC-labeled dextrans with a molecular weight of $2000 \mathrm{kDa}$ (Dex-2000, Sigma-Aldrich, $0.65 \mathrm{mg} / \mathrm{ml}$ ) was measured using the indicator dilution technique. Before the first injection of the tracer, a single bolus of $10 \mathrm{ml}$ dextrans with a molecular weight of $1 \mathrm{kDa}$ (Promiten; NPBI B.V.) was injected intracoronary to attenuate the risk for anaphylactic reactions. Within 15 minutes, a bolus injection of tracer $(1.0 \mathrm{ml})$ was given by hand into the left main via the perfusion pressure catheter at the tip of the Gregg cannula. The catheter in the great cardiac vein was connected to a roller pump enabling coronary venous blood to be collected at a rate of $24.2 \mathrm{ml}$ per minute (205S Watson Marlow), total volume of sampling system was $4 \mathrm{ml}$. Blood was sampled in consecutive $1.5 \mathrm{ml}$ tubes at intervals of $0.5-1$ second for 50 seconds. A total of 70 samples per volume measurement were obtained after the tracer injection. The first 10 and last 20 samples were taken at an interval of 1 second, while the 40 samples in between, where the major part of the indicator dilution curve is expected, were taken every half a second. Blood was subsequently centrifuged, and plasma Dex-2000 concentration was analyzed using fluorometry (Victor3; Perkin Elmer). The remaining amount of tracer after injection $(0.5 \mathrm{ml})$ was used for making calibration curves. In 12 experiments the measurement 
was repeated during intracoronary adenosine infusion $(0.47 \pm 0.03 \mathrm{mg} / \mathrm{kg} / \mathrm{h})$, to detect imposed changes in coronary plasma volume by this vasodilator. To avoid a mismatch between inflow (carotid artery) and outflow (coronary vasculature) of the perfusion system $\mathrm{P}_{\text {perf }}$ was corrected during adenosine infusion to $90-100 \mathrm{mmHg}$.

\section{Data analysis}

Analysis of the samples resulted in 70 coronary venous plasma dextran concentrations over time per coronary volume measurement. All measured data were corrected for the background value and the transfer function, $\mathrm{h}(\mathrm{t})$, was obtained by dividing the measured amount $(\mathrm{mg} / \mathrm{ml})$ times the carrier (plasma) flow $(\mathrm{ml} / \mathrm{sec})$ by the amount of injected (mg). Plasma flow was determined from coronary blood flow and hematocrit. The Dex-2000 transfer curves were fitted with four different models using Matlabß) (the Math Works, Inc., Natick, MA).

\section{Models based on the descending slope}

The monoexponential [41,42] and power-law model [4] are solely based on information obtained from a selection of the descending part of the transfer curve. The monoexponential function assumes an exponential outflow of the tracer and the power-law function assumes that the outflow can be approached as being a fractal process. For the monoexponential model the logarithm of the transfer function was taken, while for the power-law model the logarithm of the transfer function and time were both required. To obtain the model parameters, a straight line $(y=a x+b)$, using robust linear regression, was drawn through a selection of the data. Data between 70 and $30 \%$ [50] of the peak height on the descending slope of the transfer function was taken (figure 6.2a) to determine the model parameters a and b. Subsequently, for the monoexponential model, the curve was fitted (steps of 0.1 seconds), from $70 \%$ of the peak height forward, according to equation 6.1 (see table 6.1).

$$
h(t)_{e x p}=e^{a} e^{b t}=A e^{b t}
$$

Using a and b obtained from the logarithm of time and transfer function the values of $\alpha$ and $\mathrm{B}$ were determined (equation 6.2 and 6.3) after which the transfer function 
according the power-law was fitted using equation 6.4 .

$$
\begin{gathered}
\alpha=-a-1 \\
B=\frac{e^{b}}{\alpha} \\
h(t)_{P L}=B \alpha t^{-\alpha-1}
\end{gathered}
$$

All data from start of measurement up to the point of extrapolation by the models was linearly interpolated (steps of 0.1 second) to have an equal distribution of the data over the entire curve.

\section{Models based on both ascending and descending slope}

In contrast to the monoexponential and power-law models, the gamma-variate model $[81,88]$ and local density random walk model (LDRW) $[7,88]$ also take the ascending part of the curve into account. Fitting the transfer function by the gamma-variate model was done by using equation 6.5. In contrast to the more common used definition of the gamma-variate model $[46,77,88,137]$ the parameters $\left(\mathrm{h}_{\max }, \mathrm{t}_{\max }, \mathrm{t}_{0}\right.$ and $\beta$ ) in equation 6.5 are independent of each other [81] meaning that one parameter can change without having an influence on any of the other parameters. The LDRW model, equation 6.6, is the only not empirically chosen function, since all parameters $\left(\mathrm{m}, \mu, \lambda, \theta\right.$ and $\left.\mathrm{t}_{0}\right)$ have a physical meaning [88,89]. The parameters are defined as, $\mathrm{m}$ is the mass of the injected tracer, $\mu$ is the mean residence time of the tracer, $\theta$ is the flow of the carrier, $\lambda$ is a parameter related to the skewness or asymmetry of the curve and $t_{0}$ represents the zero time of the distribution.

$$
\begin{gathered}
h(t)_{\gamma}=h_{\max } t_{\max }^{-\beta} e^{\beta}\left(t-t_{0}\right)^{\beta} e^{\frac{-\beta t}{t_{\max }}} \\
h(t)_{L D R W}=\frac{m}{\mu \theta} e^{\lambda} \sqrt{\frac{\lambda \mu}{2 \pi\left(t-t_{0}\right)}} e^{\frac{-\lambda}{2}\left(\frac{\left(t-t_{0}\right)}{\mu}+\frac{\mu}{\left(t-t_{0}\right)}\right)}
\end{gathered}
$$

The parameters of the equations were determined by reducing the mean square error between the model and the measured data. Data between $5 \%$ of the peak height on the ascending slope up to $30 \%$ of the maximum peak value on the descending slope of the 


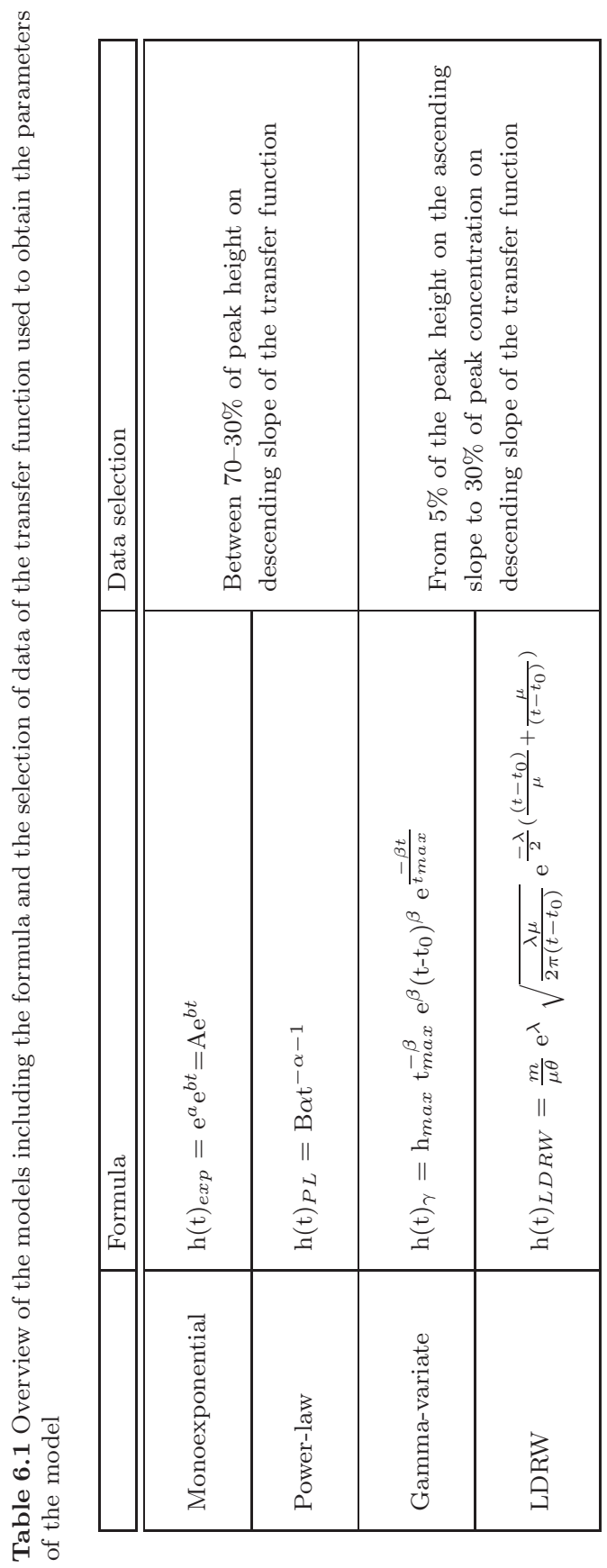


transfer function curve were compared with the data obtained from the model $[87,88]$, figure $6.2 \mathrm{~b}$. To determine how well the fit corresponds with the measured data, the determination coefficient, $\mathrm{R}^{2}$, was calculated over the selection used to determine the model parameters.

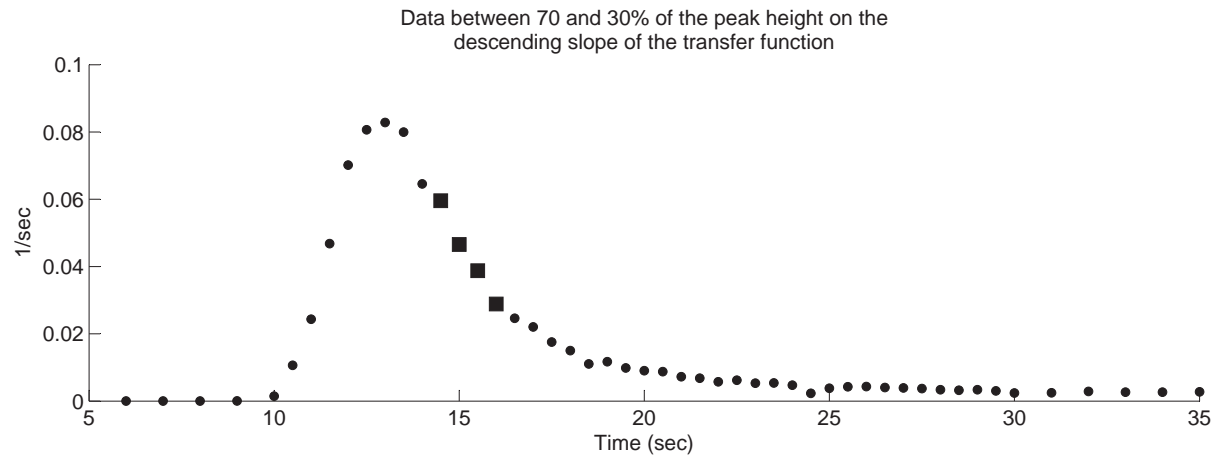

(a) Monoexponential and power-law model

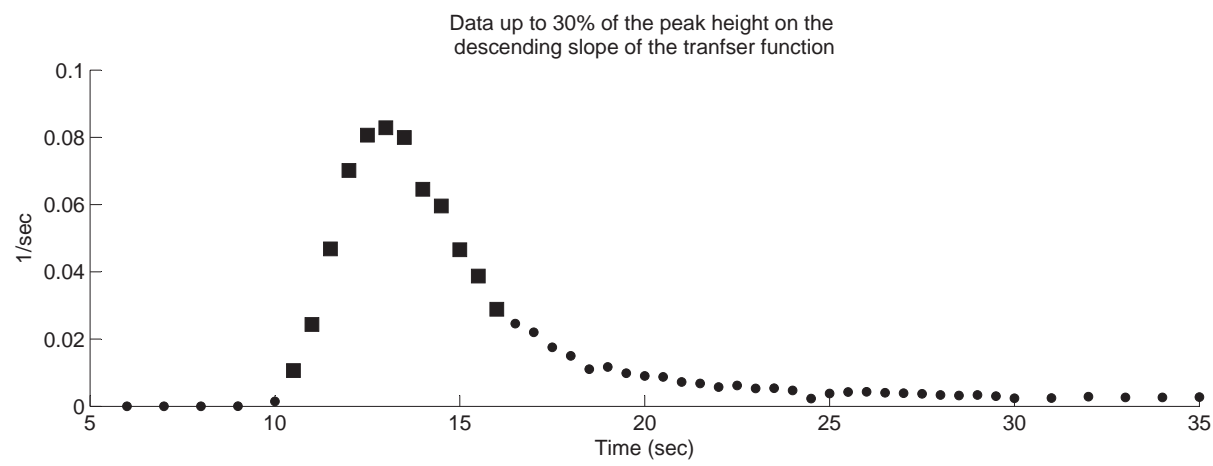

(b) Gamma-variate and LDRW model

Figure 6.2 An example of a transfer function with the different selections of data used to determine the parameters of the models. The measured data is indicated with dots $(\bullet)$, the data used by the models are marked by closed squares ( $\mathbf{\square})$. Data not yet corrected for the transit time of the sampling catheter (9.9 seconds). (a) Monoexponential and power-law model: data between 70-30\% of the peak height on the descending slope of the transfer function is taken into account. (b) Gamma-variate and LDRW model: all data between $5 \%$ of the peak height on the ascending slope up to $30 \%$ of the peak height on the descending slope of the transfer function is selected. 


\section{Calculation of coronary plasma volume}

Using the data from the fitted curve the mean transit time (MTT) was calculated according to equation 6.7, in which $\mathrm{C}(\mathrm{t})$ is the fitted Dex-2000 outflow concentration.

$$
M T T=\frac{\int t C(t) d t}{\int C(t) d t}
$$

The MTT of the tracer was corrected for the volume of the sampling catheter by subtraction of sampling transit time $(9.9 \mathrm{sec})$, i.e. dividing the sampling catheter volume ( $4 \mathrm{ml}$ ) by the sampling flow $(24.2 \mathrm{ml} / \mathrm{min})$. Multiplying the corrected MTT with the tracer carrier flow (plasma flow) gave the distribution volume of the tracer [84].

Differences in MTT, volume and determination coefficient between models were tested using a t-test, as well as differences between measurements at baseline and during adenosine infusion. Results were considered statistically significant with $\mathrm{P}<0.05$. Summary data are reported as means \pm SEM.

\section{Results}

Hemodynamic parameters of the measurements are presented in table 6.2. During the infusion of adenosine in the left main coronary artery $\mathrm{Q}_{\text {perf }}$ was significantly increased, even though the perfusion pressure was reduced $(\mathrm{P}<0.05)$. All other parameters remained unaltered during intracoronary adenosine infusion.

Table 6.2 Hemodynamic parameters

\begin{tabular}{|l|c|c|}
\hline & Baseline $(\mathrm{N}=14)$ & Adenosine $(\mathrm{N}=12)$ \\
\hline \hline $\mathrm{P}_{l v}(\mathrm{mmHg})$ & $114.8 \pm 11.4$ & $114.5 \pm 11.2$ \\
$\mathrm{P}_{\text {fem }}(\mathrm{mmHg})$ & $94.7 \pm 12.5$ & $87.7 \pm 11.8$ \\
$\mathrm{Q}_{\text {perf }}(\mathrm{ml} / \mathrm{min} / 100 \mathrm{gr})$ & $225.7 \pm 25.5$ & $388.4 \pm 27.2^{*}$ \\
$\mathrm{P}_{\text {perf }}(\mathrm{mmHg})$ & $128.0 \pm 4.0$ & $93.6 \pm 4.7^{*}$ \\
$\mathrm{HR}$ (beats/min) & $132.1 \pm 7.5$ & $129.7 \pm 8.7$ \\
Hct (venous)(\%) & $23.3 \pm 2.1$ & $22.5 \pm 1.9$ \\
\hline
\end{tabular}

Values are mean $\pm S E M,{ }^{*}$ significant from corresponding baseline measurement $(P<0.05)$. 
The prevailing flows $(225.7 \pm 25.5 \mathrm{ml} / \mathrm{min} / 100 \mathrm{gr}$, range $63.5-424.6 \mathrm{ml} / \mathrm{min} / 100 \mathrm{gr}$ at baseline and $388.4 \pm 27.2 \mathrm{ml} / \mathrm{min} / 100 \mathrm{gr}$, range $236.0-539.5 \mathrm{ml} / \mathrm{min} / 100 \mathrm{gr}$ during adenosine) resulted in an estimated time of onset of recirculation, determined from the volume of the perfusion system $(\sim 110 \mathrm{ml})$ divided by the coronary flow, of 42.6 \pm 5.5 seconds, with a range of 20.7-95.5 seconds at baseline and $22.7 \pm 1.9$ seconds (range 13.3-33.4 seconds) during adenosine infusion.

Figure 6.3 shows an example of a transfer function during baseline conditions as fitted by the four models, i.e. exponential (6.3a), power-law (6.3b), gamma-variate (6.3c) and LDRW (6.3d). For the monoexponential and power-law model there was one experiment during adenosine in which not enough data was within the selected part of the curve to perform robust regression. This experiment was, therefore, not included when the MTT and volume of the different models were compared.

Table 6.3 Determination coefficients

\begin{tabular}{|c|c|c|}
\hline & Baseline $(\mathrm{N}=14)$ & Adenosine $(\mathrm{N}=12)$ \\
\hline \hline Gamma-variate model & $0.97 \pm 0.01$ & $0.94 \pm 0.02$ \\
LDRW & $0.97 \pm 0.01$ & $0.94 \pm 0.02$ \\
\hline
\end{tabular}

Values are mean $\pm S E M$.

The accuracy of fitting of the indicator dilution curves with the gamma-variate model provided results similar to those of the LDRW model. The average determination coefficients were at baseline $0.97 \pm 0.01$ and $0.97 \pm 0.01$ for the gamma-variate and LDRW model, respectively, see table 6.3. During adenosine the $\mathrm{R}^{2}$ was reduced to $0.94 \pm 0.02$ for both models, not significantly different from baseline.

When taking all curves in consideration, there were two differences comparing the results of the monoexponential and power-law model with the results of the gammavariate and LDRW model. First, the fits by the monoexponential and power-law model appeared to contain more irregularities since they did not fit the entire (ascending and descending) curve, see figure 6.4. Secondly, the gamma-variate and LDRW model fitted curves through the data appeared to have a steeper descending slope than the actual measured curves, resulting in a visible deviation of the fit from actual measurements at the lower part of the curve (see figure $6.3 \mathrm{c}$ and $6.3 \mathrm{~d}$ ). The lower part of the descending slope of the monoexponential fit and, even more so, the power-law model derived fit correspond better with the measured data. 


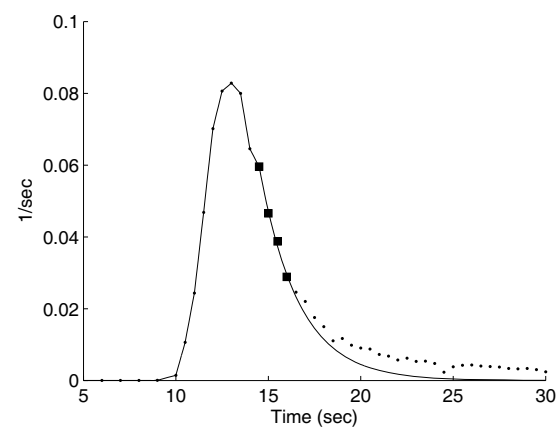

(a) Monoexponential model



(c) Gamma-variate model

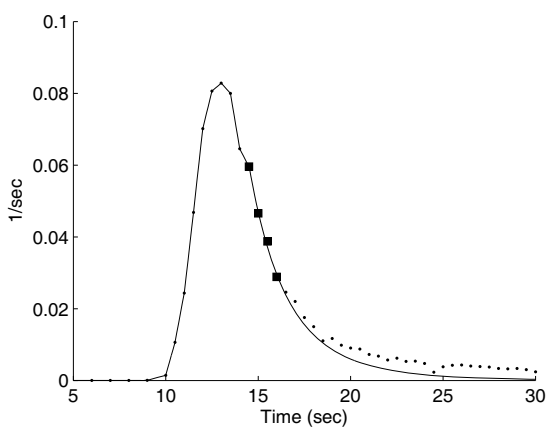

(b) Power-law model

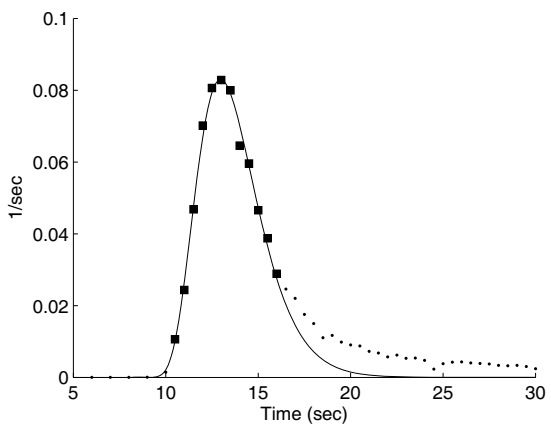

(d) Local density random walk model

Figure 6.3 Example of a transfer function fitted with four different models (a) monoexponential, (b) power-law, (c) gamma-variate, and (d) local density random walk model. The continuous lines are fitted curves based on the data between 70 and $30 \%$ of the height of the peak on the descending slope of the transfer function (6.3a and 6.3b) and all data up to $30 \%$ of the peak height on the descending slope of the transfer function (6.3c and $6.3 \mathrm{~d})$. The data used by the models are marked by closed squares (ם). Data not yet corrected for the transit time of the sampling catheter (9.9 seconds). Onset of recirculation was estimated at 49.6 seconds (additional delay of the sampling catheter not taken into account).

The derived mean transit times of the paired data are given in figure 6.5. For both, baseline and during adenosine, the MTT derived using the power-law model (6.9 \pm 0.7 and $5.9 \pm 0.5$ seconds, respectively) was significantly larger than the MTT determined using the other models. The monoexponential derived MTT (5.6 \pm 0.5 at baseline and $4.8 \pm 0.3$ seconds during adenosine) was significantly larger than the 


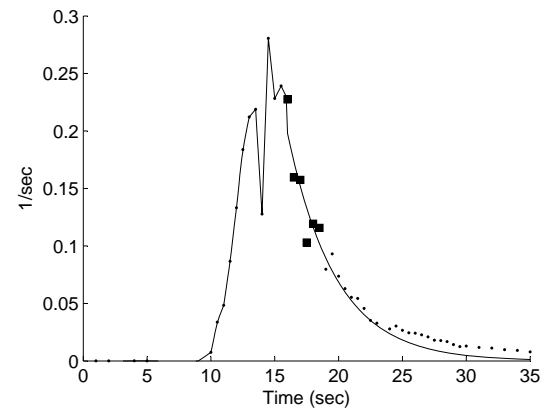

(a) Monoexponential model

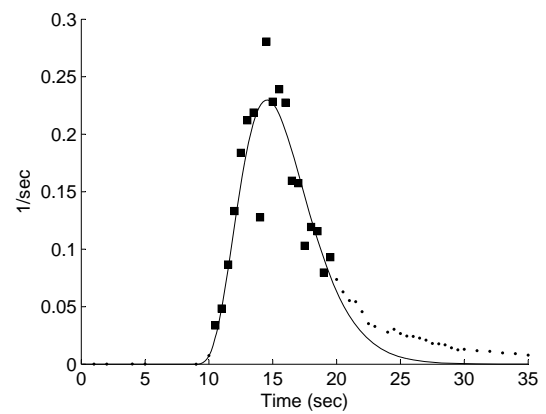

(c) Gamma-variate model

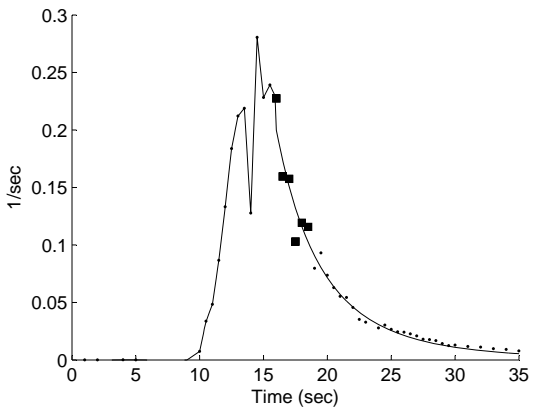

(b) Power-law model



(d) Local density random walk model

Figure 6.4 Example of a transfer function fitted with four different models, (a) monoexponential, (b) power-law, (c) gamma-variate, and (d) local density random walk model. The continuous lines are fitted curves based on the data between 70 and $30 \%$ of the height of the peak on the descending slope of the transfer function $(6.4 \mathrm{a}$ and $6.4 \mathrm{~b})$ and all data up to $30 \%$ of the peak height on the descending slope of the transfer function (6.4c and $6.4 \mathrm{~d})$. The data used by the models are marked by closed squares $(\boldsymbol{\square})$. Data not yet corrected for the transit time of the sampling catheter (9.9 seconds). Onset of recirculation was estimated at 35.1 seconds (additional delay of the sampling catheter not taken into account).

gamma-variate derived MTT (5.0 \pm 0.4 at baseline and $4.4 \pm 0.3$ seconds during adenosine) but only at baseline was it significant larger than the MTT of the LDRW model ( $5.1 \pm 0.4$ at baseline and $4.6 \pm 0.4$ seconds during adenosine). The MTT of the gamma-variate model was during adenosine smaller than all other mean transit times $(\mathrm{P}<0.05)$. 


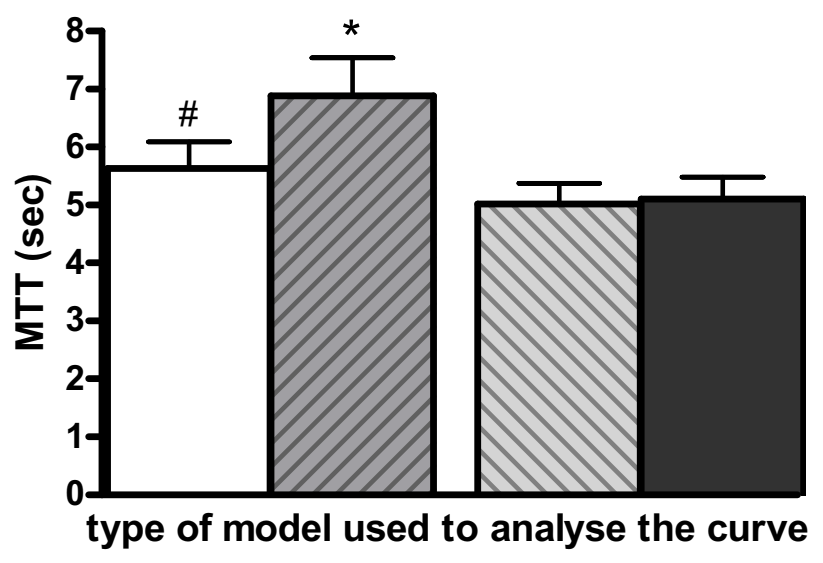

$\square$ Monoexponential

Power-law

$\square$ Gamma-variate

$\square$ LDRW

(a) Baseline



$\square$ Monoexponential

ZPower-law

$\square$ Gamma-variate

$\square$ LDRW

(b) Adenosine

Figure 6.5 The MTT (N=11) for all four models at baseline (a) and during adenosine infusion (b). * $\mathrm{P}<0.05$ compared to monoexponential, gamma-variate and LDRW model, \# $\mathrm{P}<0.05$ compared to power-law, gamma-variate and LDRW model, $\S \mathrm{P}<0.05$ compared to power-law and gamma-variate model, $\dagger \mathrm{P}<0.05$ compared to gamma-variate model. 


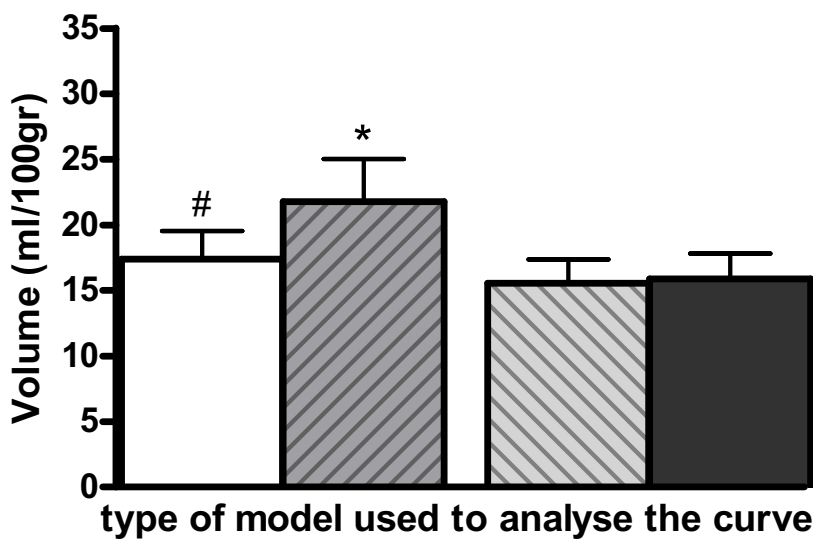

$\square$ Monoexponential

Z7 Power-law

Q.Vamma-variate

$\square$ LDRW

(a) Baseline

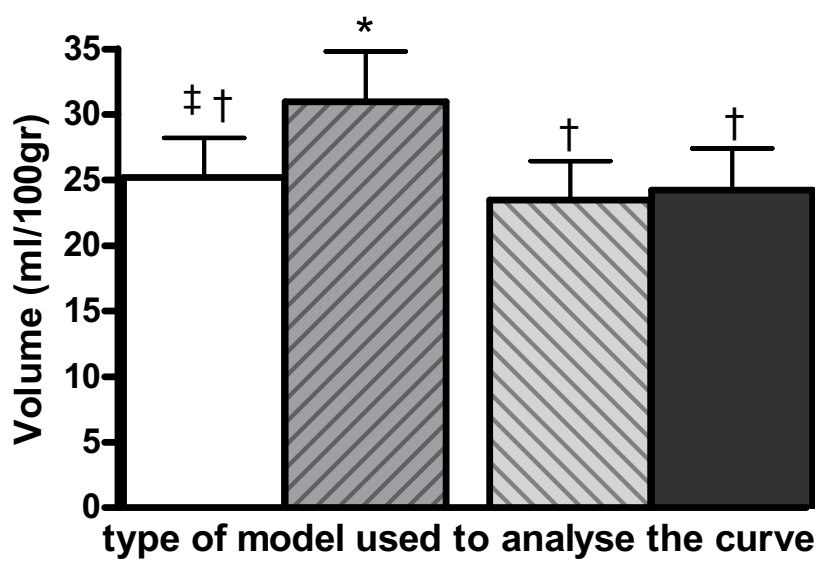

$\square$ Monoexponential

Z7. Power-law

Q.Vamma-variate

$\square$ LDRW

(b) Adenosine

Figure 6.6 The coronary plasma volumes per 100 gram heart tissue $(\mathrm{N}=11)$ for all four models at baseline (a) and during adenosine infusion (b). * $\mathrm{P}<0.05$ compared to monoexponential, gammavariate and LDRW model, \# $\mathrm{P}<0.05$ compared to power-law, gamma-variate and LDRW model, $\ddagger \mathrm{P}<0.05$ compared to power-law and gamma-variate model, $\dagger \mathrm{P}<0.05$ compared to corresponding baseline measurement. 
The paired volume estimates (MTT times the coronary plasma flow) are in figure 6.6. The coronary plasma volume per 100 gram heart tissue determined using the power-law model $(21.8 \pm 3.2 \mathrm{ml} / 100 \mathrm{gr}$ [range $6.2-40.7 \mathrm{ml} / 100 \mathrm{gr}$ ] at baseline and 31.0 $\pm 3.8 \mathrm{ml} / 100 \mathrm{gr}$ [range $15.8-55.1 \mathrm{ml} / 100 \mathrm{gr}$ ] during adenosine, $\mathrm{P}<0.06$ ) was significantly larger than the volumes estimated using the other three models. Further, the monoexponential model derived coronary plasma volume $(15.5 \pm 2.0 \mathrm{ml} / 100 \mathrm{gr}$ [range $5.8-31.7 \mathrm{ml} / 100 \mathrm{gr}$ ] at baseline and $25.2 \pm 3.0 \mathrm{ml} / 100 \mathrm{gr}$ [range 12.1-41.7 ml/100gr] during adenosine, $\mathrm{P}<0.05)$ was significant larger than those from the gamma-variate $(13.9 \pm 1.7 \mathrm{ml} / 100 \mathrm{gr}$ [range $5.3-27.7 \mathrm{ml} / 100 \mathrm{gr}$ ] at baseline and $22.5 \pm 3.0 \mathrm{ml} / 100 \mathrm{gr}$ [9.6-40.1 ml/100gr] during adenosine, $\mathrm{P}<0.01)$ and at baseline from the LDRW model $(14.2 \pm 1.8 \mathrm{ml} / 100 \mathrm{gr}$ [range $5.3-28.3 \mathrm{ml} / 100 \mathrm{gr}$ ] at baseline and $23.2 \pm 3.1 \mathrm{ml} / 100 \mathrm{gr}$ [range 9.7-42.2 ml/100gr] during adenosine, $\mathrm{P}<0.01$ ).

\section{Discussion}

In the present study, four indicator dilution models were compared for determination of coronary plasma volumes in goat hearts. Significant differences in obtained MTTs and volumes were found between the models, indicating the importance of the chosen model in determination of coronary volume when using tracer dilution.

By injection of an indicator at the entrance of the heart (coronary left main artery) and measuring the concentration in time at the output of the heart (great cardiac vein) the average time it takes for the indicator to travel through the heart (MTT) can be determined. Multiplying the MTT with the flow gives the distribution volume of the indicator. When applying the indicator dilution method to determine blood volume, the following assumptions are made: 1) flow is stationary; 2) there is a single input/output channel; 3) indicator particle flow is representative for flow of total fluid; 4) there is no recirculation; 5) flow and volume are constant in time; 6) there are no stagnant pools present $[84,170]$. One of the assumptions, no recirculation, is normally violated in in vivo measurements. In the current study we used a perfusion system with a relative large volume to delay the moment recirculation started to interfere with the first pass outflow curve. The estimated moment of recirculation was calcu- 
lated to range from a minimum of 13.3 to a maximum of 95.5 seconds. The volume in the body, from great cardiac vein to carotid artery, is not taken into account, and the calculated delay of recirculation might even be an underestimation of the actual delay. Recirculation appeared in this study at least 5 seconds after the last sample that was selected for the determination of the model parameters $(7.8 \pm 0.5$ and $7.1 \pm$ 0.4 seconds at baseline and during adenosine, respectively). Therefore, no interference of recirculation with the fits is expected in the current study.

The estimated coronary plasma volumes $(\mathrm{N}=14)$ at baseline by the monoexponential model in the current study $(15.5 \pm 2.0 \mathrm{ml} / 100 \mathrm{gr}$ at a flow of $225.7 \pm 25.5$ $\mathrm{ml} / \mathrm{min} / 100 \mathrm{gr}$ ) compare very well with values reported in the literature obtained by using the indicator dilution method in combination with a monoexponential model (15.0 ml/100gr when extrapolated to a flow of $225 \mathrm{ml} / \mathrm{min} / 100 \mathrm{gr}$ [168] and 15.6 $\mathrm{ml} / 100 \mathrm{gr}[49,91])$. As far as we know, there have been no reports in the literature of indicator dilution based coronary volumes during maximal vasodilation using adenosine. We found that the increase in coronary plasma volume during adenosine compared to baseline volume was not different comparing the models, a $1.6 \pm 0.1$ fold increase in volume. This increase in coronary plasma volume measured corresponds well with reported coronary volume increases during adenosine induced vasodilation measured using X-ray contrast enhancement (1.68 fold increase) [51] and myocardial radioactivity (1.75 fold increase) [22]. For all models, except the power-law model, was the increase in coronary blood volume during adenosine significant. The monoexponential, gamma-variate and LDRW model seemed, therefore, equally able to detect an imposed increase in coronary plasma volume and relative effects on volumes appeared model independent.

Certainly, based on literature it cannot be determined which model provides the most accurate volume estimations. Bassingthwaighte \& Beard [4] compared the monoexponential and power-law model for analyzing myocardial water washout in isolated rabbit hearts. Unfortunately, the indicator used in that study, ${ }^{15} \mathrm{O}$-labeled water, was not an intravascular tracer, what might have resulted in different shaped outflow curves as when an intravascular tracer was used. They found that the use of a powerlaw model led to an improvement of extrapolation of downslopes of indicator dilution curves. Comparing the power-law model results with the results of the monoexponential model, the monoexponential model provided underestimations of the tails of the 
curves in their study. In agreement, in the current study the coronary plasma volume estimations of the power-law model were larger than those of the monoexponential model. Furthermore, comparing the measured data with the fits of the models, the fit of the power-law model corresponded in general better with the measured tail of the curve than the fit of the monoexponential model (figure 6.3 and 6.4). However, no volume or flow measurement has ever been done using the power-law model, in vivo or in vitro. Therefore, whether the longer tails of the power-law model provide more accurate intravascular volume estimations has not yet been determined. The baseline coronary plasma volumes estimated using the two models taking the ascending part of the curve into account as well (gamma-variate and LDRW model), were significantly smaller than the volumes calculated using the monoexponential model, and consequently also the power-law model. Similar observations are made by Milard [87] and Thompson and coworkers [137]. The smaller mean transit times, and consequently volumes, for the gamma-variate as well as LDRW model can be explained by the difference in descending slope of the curve compared with the other models. The downward slope of the curve of the models taking the ascending part of the curve into account was steeper, resulting in a smaller MTT (figure 6.3 and 6.4). Although the data used to determine the model parameters were described accurately (see figure 6.3), the gamma-variate and LDRW model seemed not capable of describing the rest of the tail as good as the monoexponential and, in particular, the power-law model. As a result, the gamma-variate and LDRW models might cause an underestimation of the actual volume in the current study. Indeed, Mische et al. [88] showed that the estimated volume by these models provided underestimations for increasing volumes, in their in vitro and in vivo measurements for cardiac output. In contrast to the possible underestimation of the gamma-variate and LDRW model, the exponential model has been associated in the literature with an overestimation of the mean transit time when determining cardiac output $[8,87]$.

A major advantage of the gamma-variate and LDRW model compared to the monoexponential and power-law model is that these models fit and explain not only the descending part of the transfer curve but the ascending part of the curve, the peak, and the start of the descending slope of the transfer curve as well. As a result, these fits are less sensitive to noise, see figure 6.4. Bogaard et al. [8] found that the results of the monoexponential method are seriously affected by noise in the data, introducing 
a systematic error (overestimation of MTT) which considerably reduces the accuracy of this model. On the contrary, noise in the data did not have a significant effect on the outcome of the LDRW model. In addition, the number of data points which are being used for the fit is much larger for the gamma-variate and LDRW model compared to the monoexponential and power-law model. Thus, one of the experiments could not be fitted using the monoexponential and power-law model due to a lack of sufficient data to perform robust linear regression. Selection of less data, which seems inevitable when no or a low volume perfusion system is used, seems, therefore, not an option for these models. In contrast, the other models (gamma-variate and LDRW model) require only few data points of the descending slope.

\section{Conclusion}

In our opinion, this is the first study in which the monoexponential, power-law, gamma-variate and LDRW model were compared for indicator-dilution outflow curves in the coronary circulation. The choice of model used to analyze the data influences the outcome of the study and should be chosen carefully.

Comparing the models we found that the monoexponential and especially the powerlaw model appeared to describe the tail of indicator dilution curves better than the gamma-variate and LDRW model. However, a disadvantage of both models is that not all curves could be fitted due to not having enough data within the selected part of the curve, i.e. between 30 and $70 \%$ of the peak height on the descending slope. The gamma-variate and LDRW model, which take both ascending and descending slope of the curve into account, were able to fit all curves. Furthermore, they are less sensitive to noise and fewer data are required from the descending slope of the curve to obtain good fits. Therefore, we suggest using the gamma-variate or LDRW model. 


\section{Acute attenuation of glycocalyx}

barrier properties increases coronary blood volume independently of coronary flow

reserve

The contents of this chapter are based on Judith Brands, Jos AE Spaaan, Bernard M Van den Berg, Hans Vink, and Jurgen WGE Van Teeffelen Acute attenuation of glycocalyx barrier properties increases coronary blood volume independently of coronary flow reserve (2010), American Journal of Physiology - Heart and circulatory physiology 


\section{Abstract}

Aims Vascular endothelium is covered with an extensive mesh of glycocalyx constituents, which acts like an effective barrier up to several microns thick that shields the luminal surface of the vasculature from direct exposure to flowing blood. Many studies report that various enzymatic and pharmaceutical challenges are able to increase glycocalyx porosity, resulting in farther permeation of plasma macromolecules and greater access of red blood cells into the glycocalyx domain. Attenuation of glycocalyx barrier properties therefore potentially increases the amount of blood that effectively occupies available microvascular volume. We tested in the present study whether attenuation of coronary glycocalyx barrier properties actually increases coronary blood volume and whether such changes would be noticeable during measurements of coronary flow reserve using adenosine. Methods In anesthetized goats $(\mathrm{N}=6)$ with cannulated left main coronary artery that were perfused under controlled pressure, coronary blood volume was measured via the indicator-dilution-technique using high molecular weight $(2000 \mathrm{kDa})$ dextrans as plasma tracer and labeled red blood cells as red blood cell tracer. Coronary blood volume was determined at baseline and during intracoronary infusion of adenosine causing maximal vasodilation (0.2-0.6 $\mathrm{mg} / \mathrm{kg} / \mathrm{h}$ ) before and after intracoronary hyaluronidase treatment (170.000 units) of the glycocalyx. Results With an intact glycocalyx, coronary blood volume was 18.9 $\pm 1.1 \mathrm{ml} / 100 \mathrm{gr}$ heart tissue at baseline, which increased to $26.3 \pm 2.7 \mathrm{ml} / 100 \mathrm{gr}$ after hyaluronidase treatment of the coronary glycocalyx. Maximal vasodilation by administration of adenosine further increased coronary blood volume to $33.9 \pm 6.8$ $\mathrm{ml} / 100 \mathrm{gr}$, a value not different from the maximal coronary blood volume of $33.2 \pm$ $5.3 \mathrm{ml} / 100 \mathrm{gr}$ obtained by administration of adenosine in the absence of hyaluronidase treatment. Adenosine induced increases in coronary conductance were not affected by hyaluronidase treatment. Conclusion Acute attenuation of glycocalyx barrier properties increases coronary blood volume by approximately $40 \%$, which is of similar magnitude as additional changes in coronary blood volume during subsequent maximal vasodilation with adenosine. Furthermore, maximal coronary blood volume following administration of adenosine was similar with and without prior hyaluronidase degradation of the glycocalyx, suggesting that adenosine and hyaluronidase potentially increase glycocalyx porosity to a similar extend. Hyaluronidase mediated changes 
Glycocalyx degradation uncouples coronary blood flow and volume regulation

in coronary blood volume did not affect baseline and adenosine-induced increases in coronary conductance, demonstrating that measurements of coronary flow reserve are insufficient to detect impairment of coronary blood volume recruitment in conditions of damaged glycocalyx.

\section{Introduction}

Our previous study demonstrated the presence of a thick glycocalyx in the coronary circulation [142]. The glycocalyx thickness has been estimated to be $0.2-0.9 \mu \mathrm{m}$ in capillaries $[47,100,103,110,111,121,152,157,158]$. Because of the limited access of flowing blood to an intact glycocalyx, microvascular blood volume might therefore in effect be reduced by as much as $50 \%$ of available anatomical microvascular volume due to glycocalyx presence. Others have demonstrated that enzymatic treatment of the glycocalyx with, for example, heparinase or hyaluronidase [29,47, 142] and also exposure of the glycocalyx to the vasodilators adenosine, bradykinin and sodium nitroprusside $[74,110,152,154]$ increases glycocalyx porosity for plasma macromolecules and red blood cells. However, measurements of the effect of attenuated glycocalyx barrier properties on potential increases in coronary blood volume have never been performed.

Since it was established for hyaluronidase and adenosine that the porosity of the glycocalyx increases, we hypothesize that attenuation of glycocalyx barrier properties by hyaluronidase treatment and adenosine administration increases coronary blood volume. Furthermore, in the view of the fact that adenosine is the preferred vasodilator in the clinic for evaluation of coronary flow reserve, we tested whether changes in glycocalyx porosity would be noticeable during measurement of coronary flow reserve using adenosine. In anesthetized goats, coronary blood volume was measured under control conditions and after hyaluronidase treatment at baseline and during the administration of adenosine. Coronary blood volumes were determined by applying the tracer-dilution technique, using dextrans with a molecular weight of $2000 \mathrm{kDa}$ (Dex-2000) as plasma tracer and labeled red blood cells as red blood cell tracer. 


\section{Methods}

\section{General Surgery and Anesthesia}

All procedures and protocols were approved by the animal care and use committee of Maastricht University (DECno. 2007-061). Studies were conducted in accordance with the National Institutes of Health Guide for the Care and Use of Laboratory Animals. Experiments were performed on female adult goats of $18-27 \mathrm{~kg}(\mathrm{~N}=6)$. At the beginning of an experiment, the goats were anesthetized with an intramuscular injection of Nimatek (15 mg/kg, Eurovet Animal Health BV) and Dormicum (0.75 $\mathrm{mg} / \mathrm{kg}$, Roche). Next, goats were intubated and ventilated with a 1:2 $\mathrm{O}_{2}$ :air mixture, and anesthesia was maintained by intravenous administration of Sufentanil-hameln (9.375 $\mu \mathrm{g} / \mathrm{kg} / \mathrm{h}$, Hameln Pharmaceuticals GmbH), Dormicum (0.625 mg/kg/h) and Propofol (10 mg/kg/h, Fresenius Kabi Nederland B.V.). Depth of anesthesia was adjusted according to the stability of femoral artery blood pressure $\left(\mathrm{P}_{f e m}\right)$ and heart rate $(\mathrm{HR})$. Arterial and coronary venous blood gases and $\mathrm{pH}$ were measured every 30 minutes. When necessary, ventilation was adjusted to maintain oxygen and $\mathrm{CO}_{2}$ pressures within physiological limits, and sodium bicarbonate was administered to avoid acidosis.

A left thoracotomy was performed in the fourth intercostal space. and one of the ribs was removed. The great cardiac vein was cannulated via the azygos vein to obtain coronary venous blood samples. Next, a double purse string was made in the outer wall of the aorta. Subsequently, the heart was exposed and suspended in a pericardial cradle. The left main coronary artery was dissected free, and a ligature was placed around the vessel. A catheter (7F, Sentron) connected to a Sentron interface (Type no. 811-000) was inserted through the left auricle into the left ventricle to measure pressure $\left(\mathrm{P}_{l v}\right)$. After administration of a $3 \mathrm{ml}$ heparin bolus (5000 IE/ml, LEO Pharma B.V.), anticoagulation was maintained by continuous infusion of heparin (1000 IE/h). Next, the left carotid artery was cannulated and a stainless steel Gregg cannula was inserted in the aorta via the purse string. Blood collected from the carotid artery was circulated through a perfusion system and back via the Gregg cannula into the aorta for 10 minutes. Hereafter, the Gregg cannula was ligated into the left main coronary artery, without disrupting the coronary flow. Coronary perfusion was controlled using a roller pump perfusion system [131]. Blood collected from the left carotid artery was heated 
Glycocalyx degradation uncouples coronary blood flow and volume regulation

and filtered and was circulated with a roller pump via a reservoir (70 ml) into the left main coronary artery. Blood level and pressure in the reservoir were kept constant. Perfusion pressure $\left(\mathrm{P}_{\text {perf }}\right)$ was measured at the tip of the Gregg cannula. An inline flow probe (6mm Transonic flowprobe, Transonic Systems Inc.) was interpositioned into the perfusion system to measure coronary blood flow $\left(\mathrm{Q}_{c o r}\right)$. $\mathrm{Q}_{\text {cor }}$, depending on coronary resistance, was pulsatile as a result of cardiac contraction.

$\mathrm{P}_{\text {fem }}, \mathrm{P}_{\text {perf }}, \mathrm{P}_{l v, s y s}$ (left ventricular systolic blood pressure determined from maximal $\mathrm{P}_{l v}$ per beat), $\mathrm{Q}_{c o r}$ and $\mathrm{HR}$ (determined from $\mathrm{P}_{l v}$ ) were stored for off-line analysis (IDEEQ $250 \mathrm{~Hz}$, IDEE). At the end of the experimental procedure, a battery was placed on the heart to induce ventricular fibrillation. The heart was taken out, and total heart weight as well as the weight of the perfused area was measured. Perfusion area was determined by injection of white paint into the left main coronary artery and dissection and weighing of the demarcated area.

\section{Experimental protocol}

After surgery, the preparation was allowed to equilibrate for 30 minutes. In each animal distribution volumes of two tracers, the plasma tracer FITC-labeled dextrans with a MW of $2000 \mathrm{kDa}$ (Sigma-Aldrich, $1.3 \mathrm{mg} / \mathrm{ml}$ ) and 5-(and-6)-carboxyfluorescein diacetate, succinimidyal ester (5(6)-CFDA,SE; CFSE, Invitrogen) labeled red blood cells were measured using the indicator-dilution-technique. The day before the experiment, $10 \mathrm{ml}$ blood were taken from the goat and centrifuged at $1200 \mathrm{~g}$. Plasma was removed, and $30 \mathrm{ml}$ of 5(6)-CFDA,SE tracer $(33.3 \mathrm{ng} / \mathrm{ml})$ were added to the red blood cells. The solution was kept in the dark for 5 minutes before it was centrifuged and the supernatant removed. Red blood cells were washed three times with PBS, after which $1 \mathrm{ml}$ of plasma was added to the red blood cells. Labeled red blood cells were kept at $4^{\circ} \mathrm{C}$ in the dark overnight. At the experimental day, $0.75 \mathrm{ml}$ of Dex-2000 was mixed with $0.75 \mathrm{ml}$ of labeled red blood cells for each volume measurement. Before the first injection of tracers, a single bolus of $10 \mathrm{ml}$ of dextrans with a molecular weight of $1 \mathrm{kDa}$ (Promiten; NPBI B.V.) was injected intracoronary to attenuate the risk for anaphylactic reactions. Within 15 minutes [80], a bolus injection of $1 \mathrm{ml}$ of the tracer-mix was given by hand into the left main coronary artery via the perfusion pressure catheter at the tip of the Gregg cannula. Blood samples were collected from the great cardiac vein at a rate of $24.2 \mathrm{ml}$ per minute using a roller pump (205S 
Watson Marlow). Blood was sampled in consecutive $1.5 \mathrm{ml}$ tubes at intervals of $0.5-1$ second for 50 seconds. A total of 70 samples per coronary volume measurement was obtained.

Before each volume measurement, an arterial and coronary venous blood sample was taken for determination of blood gases. Fractional myocardial oxygen extraction was defined as the arteriovenous oxygen difference as percentage of the arterial oxygen content, with the oxygen content of the blood sample being computed as $1.34 \times$ plasma hemoglobin $\times$ oxygen saturation $\times 0.01+0.0225 \times$ oxygen pressure. Myocardial oxygen consumption was defined as coronary blood flow $\times$ arteriovenous oxygen difference. In each animal, a similar order of coronary blood volume measurements was done; first, a baseline measurement was taken at a $\mathrm{P}_{\text {perf }}$ of $127.1 \pm 5.7 \mathrm{mmHg}$. Next, adenosine $(0.2-0.6 \mathrm{mg} / \mathrm{kg} / \mathrm{h})$ was infused via the perfusion system into the left main coronary artery at a concentration inducing maximal dilation (as verified by lack of reactive hyperemia after the perfusion line had been clamped for $15 \mathrm{sec}$ ) without significant changes in heart rate. During adenosine infusion, $\mathrm{P}_{\text {perf }}$ was reduced to 95.2 $\pm 3.6 \mathrm{mmHg}$. $\mathrm{P}_{\text {perf }}$ was corrected during adenosine infusion to avoid a mismatch between inflow (carotid artery) and outflow (coronary vasculature) of the perfusion system. The tracer mixture was injected at least 10 minutes after the start of adenosine infusion to allow for a new hemodynamic steady-state. Infusion of adenosine was stopped after the last coronary venous sample was collected. When $\mathrm{Q}_{\text {cor }}$ had returned to baseline (5-10 $\mathrm{min}), \mathrm{P}_{\text {perf }}$ was increased to its initial value $(118.6 \pm 8.2$ $\mathrm{mmHg}$ ) and infusion of $100 \mathrm{ml}$ hyaluronidase was started (1700 units/ml, type IV-S, Sigma-Aldrich; intracoronary). Following 50 minutes of hyaluronidase infusion, a second baseline measurement was performed. The fourth measurement was performed during infusion of adenosine at $\mathrm{P}_{\text {perf }}$ of $92.3 \pm 6.1 \mathrm{mmHg}$. For reference, three additional experiments were performed in which measurements were done using phosphate buffered saline (vehicle) in stead of hyaluronidase to determine reproducibility of the baseline coronary blood volume measurement after adenosine washout. Collected blood was analyzed using a FACSCalibur Flow Cytometer (BD Biosciences) for labeled red blood cell dilution and subsequently centrifuged. Plasma Dex-2000 concentration was analyzed using fluorometry (Victor3; Perkin Elmer). The remaining amount of tracer-mix after injection $(0.5 \mathrm{ml})$ was used for making calibration curves. 
Glycocalyx degradation uncouples coronary blood flow and volume regulation

From $\mathrm{N}=6$ experiments, the hyaluronan levels in the plasma determined for baseline, during adenosine and after hyaluronidase $(\mathrm{N}=3)$ or vehicle $(\mathrm{N}=3)$ were given using a hyaluronan ELISA (Echelon). Removal of the glycocalyx by hyaluronidase was confirmed by Alcian blue 8GX-staining of the glycocalyx in two additional goat hearts (one control and one hyaluronidase). In essence, staining was performed as described for the isolated rat heart [142], except for the left main coronary perfusion (using the perfusion system). Cardiac tissue analysis was performed in an identical manner as described for the rats.

\section{Data analysis}

\section{Determination of tracer mean transit time}

Per coronary blood volume measurement, 70 coronary venous plasma dextran concentrations and labeled red blood cell fractions in time were obtained. Dex-2000 concentration and labeled red blood cell fraction curves versus time were fitted with a probability density function, the local density random walk (LDRW) [7], see equation 7.1. To overcome contamination of the output signal by recirculating tracer, information from the curves was used up to $15 \%$ of the peak concentration on the descending slope of the curve. The selection of data up to such a low concentration of the peak concentration was allowed because of the use of the perfusion system (volume $\sim 110$ $\mathrm{ml}$ ), delaying the moment at which recirculation of tracers starts to interfere with the measured data. Fitting of the probability density function was performed with a non-linear least squares fitting procedure.

$$
C(t)=\frac{m}{\mu \theta} e^{\lambda} \sqrt{\frac{\lambda \mu}{2 \pi\left(t-t_{0}\right)}} e^{\frac{-\lambda}{2}\left(\frac{\left(t-t_{0}\right)}{\mu}+\frac{\mu}{\left(t-t_{0}\right)}\right)}
$$

Parameters are defined as, $\mathrm{m}$ is the mass of the injected tracer, $\mu$ is the mean residence time of the tracer, $\theta$ is the flow of the carrier, $\lambda$ is a parameter related to the skewness or asymmetry of the curve, and $t_{0}$ represents the zero time of the distribution. Figure 7.1 shows an example of obtained concentration curves and LDRW fits of Dex-2000 versus time (left) and labeled red blood cell fraction curves versus time (right) for baseline (black) and adenosine (gray), before (top) and after (bottom) hyaluronidase. 


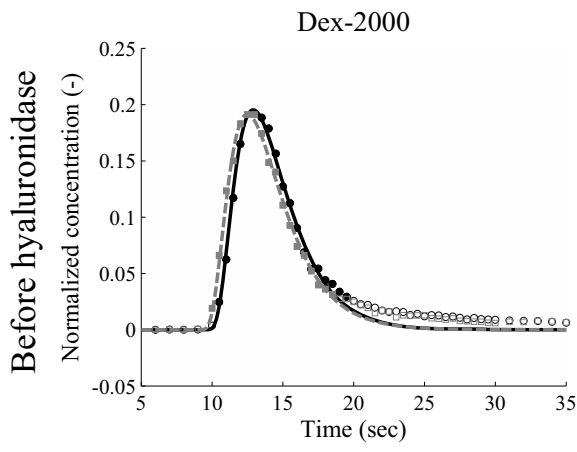

(a) Dex-2000 before hyaluronidase

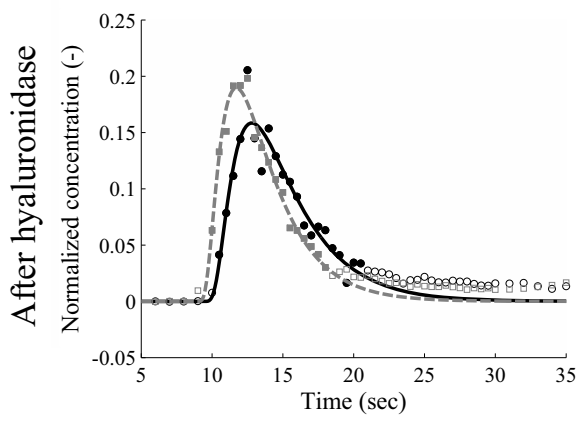

(c) Dex-2000 after hyaluronidase

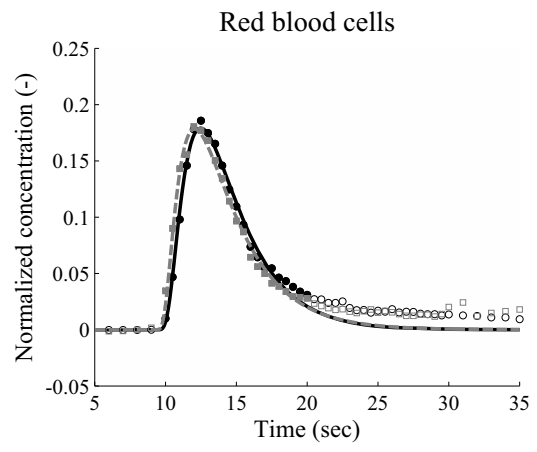

(b) Red blood cells before hyaluronidase

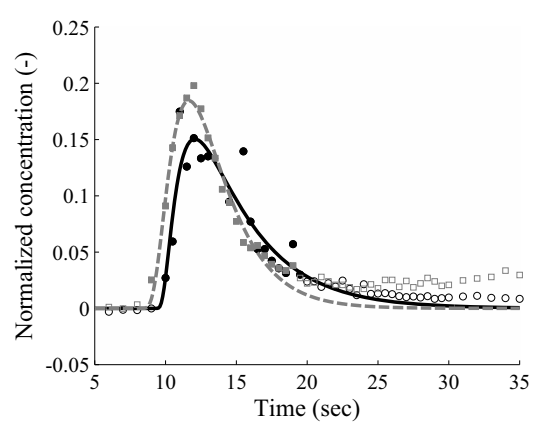

(d) Red blood cells after hyaluronidase

Figure 7.1 Example of obtained concentration curves versus time of Dex-2000 (left) and labeled red blood cells fractions versus time (right) for baseline $(\bullet)$ and concomitant LDRW-fit (black line) and for adenosine ( $\square$ ) and concomitant LDRW-fit (gray dashed line) before (top) and after (bottom) hyaluronidase. Measured labeled fraction and concentration are normalized to the area under the curve. Per measurement, 70 blood samples were taken from the great cardiac vein, and all data were analyzed using a model (LDRW). Data up to $15 \%$ of the peak value on the descending slope of the curve were used to obtain the model parameters. The Dex-2000 MTTs in this animal were, after correction for volume of sampling catheter (sampling transit time of 9.9 seconds), 4.5 seconds at baseline, 4.1 seconds during adenosine, 5.2 seconds at baseline after hyaluronidase and 3.8 seconds after additional adenosine infusion, respectively. The red blood cell MTTs at baseline and adenosine before and after hyaluronidase were 4.6, 4.4, 5.2 and 3.4 seconds, respectively.

Using data from the fitted curve the mean transit time (MTT) of each indicator was calculated according to equation 7.2, in which $\mathrm{C}(\mathrm{t})$ is the fitted Dex-2000 outflow 
Glycocalyx degradation uncouples coronary blood flow and volume regulation

concentration or labeled red blood cell fraction.

$$
M T T=\frac{\int t C(t) d t}{\int C(t) d t}
$$

Tracer MTT was corrected for sampling catheter volume by subtraction of sampling transit time (i.e., $9.9 \mathrm{sec}$ ).

\section{Coronary blood volume and conductance}

Multiplying the corrected MTT with the tracer carrier flow gives the distribution volume of each tracer [84]. Plasma and red blood cell flow were determined from coronary venous hematocrit, determined before each volume measurement, and $\mathrm{Q}_{c o r}$. Coronary blood volume (V) was defined as the sum of calculated Dex-2000 and red blood cell volume. To correct for an influence of perfusion pressure on coronary flow, coronary conductance $(\mathrm{C})$ was calculated as the ratio of $\mathrm{Q}_{\text {cor }}$ over $\mathrm{P}_{\text {perf }}$ during the 50 seconds when samples were taken after tracer mixture injection.

\section{Statistics}

The effect of time on blood pressure, HR and coronary venous hematocrit was analyzed using a Friedman test with Dunn's multiple comparison as post-hoc test. Effects of adenosine and hyaluronidase on hemodynamics, myocardial oxygen extraction, MTT, volume and conductance were analyzed with a paired Wilcoxon signed rank test. The effect of hyaluronidase and vehicle on changes in myocardial oxygen extraction and myocardial oxygen consumption from baseline to adenosine was tested using a t-test. Results were considered statistically significant with $\mathrm{P}<0.05$. Summary data are reported as means \pm SEM.

\section{Results}

During the infusion of adenosine, hyaluronan levels increased from $3407 \pm 629 \mathrm{ng} / \mathrm{ml}$ to $5944 \pm 1432 \mathrm{ng} / \mathrm{ml}$, an increase of $89 \pm 39 \%$ compared with baseline. After subsequent hyaluronidase $(\mathrm{N}=3)$, an additional increase in hyaluronan of $3871 \pm 2271$ $\mathrm{ng} / \mathrm{ml}(92 \pm 57 \%)$ was observed compared with the concentration of these samples 
during adenosine. In contrast, when the vehicle was given there was no additional increase $(7 \pm 19 \%)$ in hyaluronan levels.

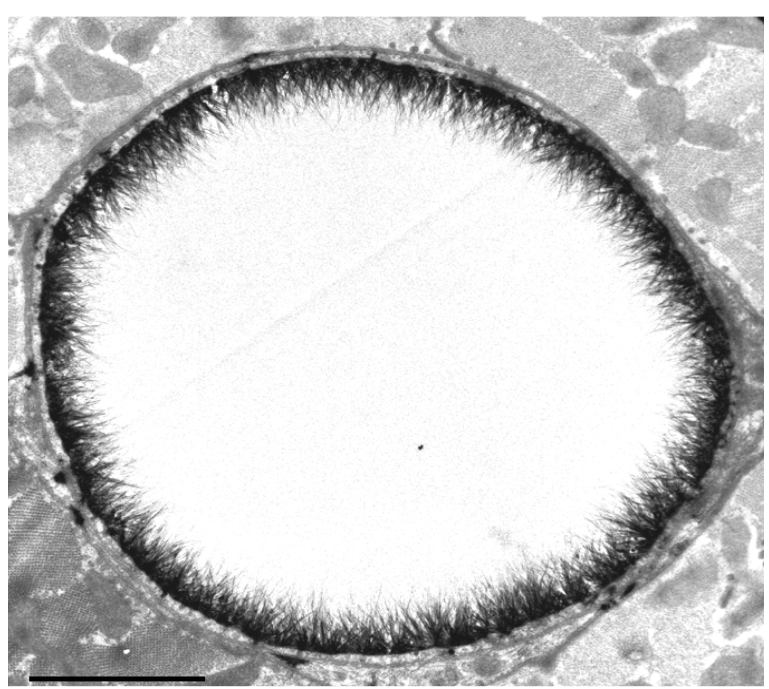

(a) Control

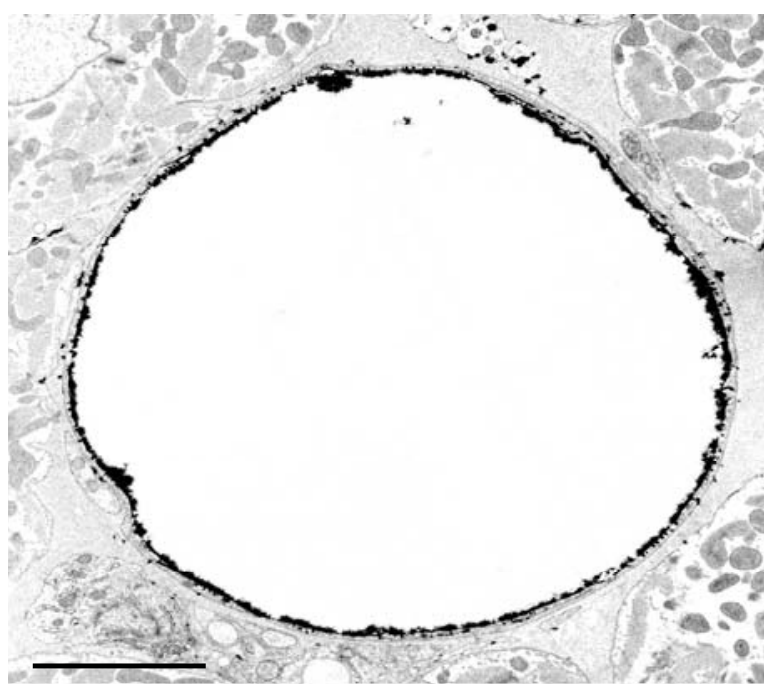

(b) Hyaluronidase

Figure 7.2 Electron microscopic overview of Alcian blue 8GX-stained goat coronary capillary before (a) and after (b) hyaluronidase. Scale bar length is $1 \mu \mathrm{m}$. 
Degradation of glycocalyx structures after hyaluronidase treatment is illustrated by the electron microscopic images in figure 7.2. Whereas in the control goat heart (7.2a), abundant polysaccharide structures of the glycocalyx are present on the luminal side of the endothelium, these structures appear to be lost in the hyaluronidase treated heart (7.2b). These observations confirm previous observations of severe glycocalyx degradation with hyaluronidase in isolated rat hearts [142].

Table 7.1 Hemodynamic parameters at baseline and during adenosine before and after hyaluronidase $(\mathrm{N}=6)$ or vehicle $(\mathrm{N}=3)$

\begin{tabular}{|c|c|c|c|c|}
\hline & \multicolumn{2}{|c|}{ Before hyaluronidase } & \multicolumn{2}{|c|}{ After hyaluronidase } \\
\hline & Baseline & Adenosine & Baseline & Adenosine \\
\hline $\mathrm{P}_{l v, s y s}(\mathrm{mmHg})$ & $101.5 \pm 5.9$ & $95.2 \pm 8.7$ & $90.9 \pm 4.7$ & $79.5 \pm 6.6$ \\
\hline $\mathrm{P}_{\text {fem }}(\mathrm{mmHg})$ & $87.7 \pm 9.6$ & $79.9 \pm 11.1$ & $75.5 \pm 5.9$ & $57.9 \pm 6.9^{\#}$ \\
\hline $\mathrm{Q}_{\text {perf }}(\mathrm{ml} / \mathrm{min} / 100 \mathrm{gr})$ & $248.3 \pm 14.5$ & $441.1 \pm 33.9^{*}$ & $276.2 \pm 31.7$ & $468.4 \pm 49.4^{*}$ \\
\hline HR (beats/min) & $139.7 \pm 11.2$ & $151.7 \pm 12.2$ & $152.4 \pm 9.4$ & $133.2 \pm 13.5$ \\
\hline Hematocrit (\%) & $21.6 \pm 2.6$ & $21.1 \pm 2.7$ & $20.7 \pm 2.6$ & $19.9 \pm 2.6$ \\
\hline Oxygen extraction (\%) & $57.1 \pm 5.0$ & $31.5 \pm 5.4^{*}$ & $51.8 \pm 3.4$ & $22.2 \pm 3.0^{*}$ \\
\hline \multirow{3}{*}{$\begin{array}{l}\text { Myocardial oxygen } \\
\text { consumption }(\mathrm{ml} / \mathrm{min} / 100 \mathrm{gr})\end{array}$} & $11.0 \pm 0.9$ & $11.1 \pm 1.9$ & $11.4 \pm 1.3$ & $7.7 \pm 0.8^{*}$ \\
\hline & \multicolumn{2}{|l|}{ Before vehicle } & \multicolumn{2}{|l|}{ After vehicle } \\
\hline & Baseline & Adenosine & Baseline & Adenosine \\
\hline $\mathrm{P}_{l v, s y s}(\mathrm{mmHg})$ & $108.0 \pm 7.8$ & $90.4 \pm 10.0$ & $89.8 \pm 1.2$ & $75.8 \pm 1.4$ \\
\hline $\mathrm{P}_{\text {fem }}(\mathrm{mmHg})$ & $88.8 \pm 8.3$ & $64.6 \pm 10.6$ & $65.9 \pm 2.1$ & $51.9 \pm 1.5$ \\
\hline $\mathrm{Q}_{\text {perf }}(\mathrm{ml} / \mathrm{min} / 100 \mathrm{gr})$ & $188.5 \pm 26.1$ & $355.1 \pm 35.5$ & $190.4 \pm 8.4$ & $367.3 \pm 38.3$ \\
\hline HR (beats/min) & $143.6 \pm 23.7$ & $182.3 \pm 10.1$ & $166.4 \pm 8.1$ & $177.6 \pm 13.7$ \\
\hline Hematocrit (\%) & $24.2 \pm 0.7$ & $24.5 \pm 0.9$ & $22.1 \pm 2.3$ & $22.8 \pm 2.3$ \\
\hline Oxygen extraction (\%) & $58.2 \pm 6.9$ & $31.0 \pm 1.2$ & $45.7 \pm 10.3$ & $22.3 \pm 3.8$ \\
\hline $\begin{array}{l}\text { Myocardial oxygen } \\
\text { consumption }(\mathrm{ml} / \mathrm{min} / 100 \mathrm{gr})\end{array}$ & $9.2 \pm 1.3$ & $11.8 \pm 1.3$ & $9.1 \pm 2.1$ & $8.6 \pm 3.2$ \\
\hline
\end{tabular}

Values are mean \pm SEM. Systolic left ventricle pressure $\left(P_{l v, s y s}\right.$ determined from maximal $P_{l v}$ per beat), $P_{\text {fem }}, H R$, and $Q_{\text {perf }}$ were averaged over 50 seconds after tracer-mix injection. * significant from corresponding baseline $(P<0.05)$, \# significant from baseline before hyaluronidase (P<0.05; post-hoc comparison). 
Baseline hemodynamic parameters are presented in table 7.1. In six animals, volume measurements at baseline and during adenosine were repeated after hyaluronidase treatment, whereas in three additional animals, phosphate buffered saline was used instead of hyaluronidase. No difference in hemodynamic parameters was found comparing the vehicle group with animals treated with hyaluronidase. Femoral artery blood pressure was reduced in both groups $(\mathrm{P}<0.05$ for hyaluronidase and $\mathrm{P}=0.07$ for vehicle) in the second adenosine measurement, whereas left ventricular systolic blood pressure and HR were unaffected. During administration of adenosine, increases in blood flow were matched by proportional decreases in myocardial oxygen extraction (as \% of that during corresponding baseline), resulting in similar amounts of oxygen delivered to the myocardial tissue (103.7 $\pm 16.8 \%$ of baseline). In contrast, after hyaluronidase mediated degradation of the glycocalyx, oxygen consumption during administration of adenosine was significantly reduced to $68.3 \pm 4.3 \%$ of corresponding baseline due to disproportional reduction of oxygen extraction (table 7.1). In the vehicle group, oxygen consumption during adenosine was not different before or after vehicle infusion.

MTTs of tracer outflow curves were derived from fitting the coronary venous concentration-time curve for both tracers with a LDRW fit (see Methods, figure 7.1). After hyaluronidase, there was a trend for an increase in MTT for both tracers (from $4.8 \pm 0.4$ to $6.1 \pm 0.7 \mathrm{sec}$ for Dex-2000 and $4.6 \pm 0.5$ to $5.9 \pm 0.5 \mathrm{sec}$ for red blood cells, both $\mathrm{P}=0.06)$. MTTs tended to decrease $(\mathrm{P}=0.06)$ when adenosine was subsequently administered $(4.3 \pm 0.5$ and $4.3 \pm 0.7 \mathrm{sec}$ for Dex-2000 and red blood cells, respectively). In contrast, with an intact glycocalyx, the MTT of both dextrans and

Figure 7.3 (following page) Absolute (a) and normalized to baseline (b) coronary blood volumes at baseline (base) and during maximal vasodilation with adenosine (ado), before and after hyaluronidase (hyal). After hyaluronidase, there was a $39 \pm 13 \%$ increase in coronary blood volume due to a 41 $\pm 16 \%$ increase in Dex-2000 distribution volume and a $34 \pm 6 \%$ increase in red blood cell volume (not different from the increase in Dex-2000 volume). Hyaluronidase treatment had no effect on adenosine-induced maximal coronary blood volume. During adenosine, coronary blood volume was $177.3 \pm 27 \%$ of coronary blood volume at baseline before hyaluronidase treatment and $181.1 \pm 35 \%$ after hyaluronidase treatment. The increase in coronary blood volume after hyaluronidase is due to recruitment of glycocalyx volume. The additional increase in coronary blood volume during adenosine is suggested to be caused by vasodilation of the resistance vessels. With an intact glycocalyx, the increase in coronary blood volume is suggested to be due to glycocalyx recruitment and vasodilation (areas based on data measured after hyaluronidase before and during adenosine administration). * $\mathrm{P}<0.05$ from baseline volume measurement before hyaluronidase. 


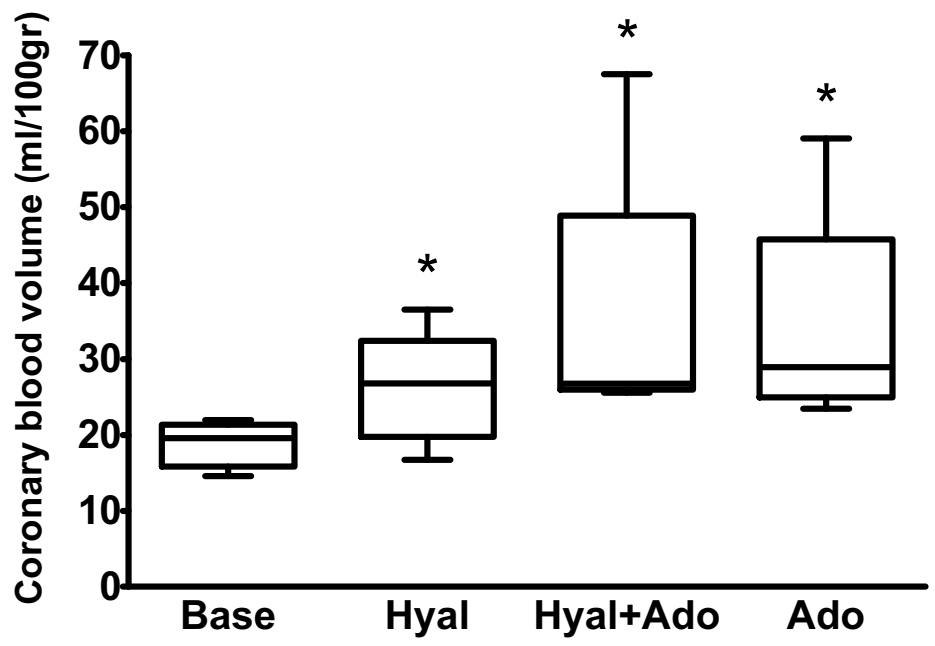

(a) Absolute coronary blood volumes

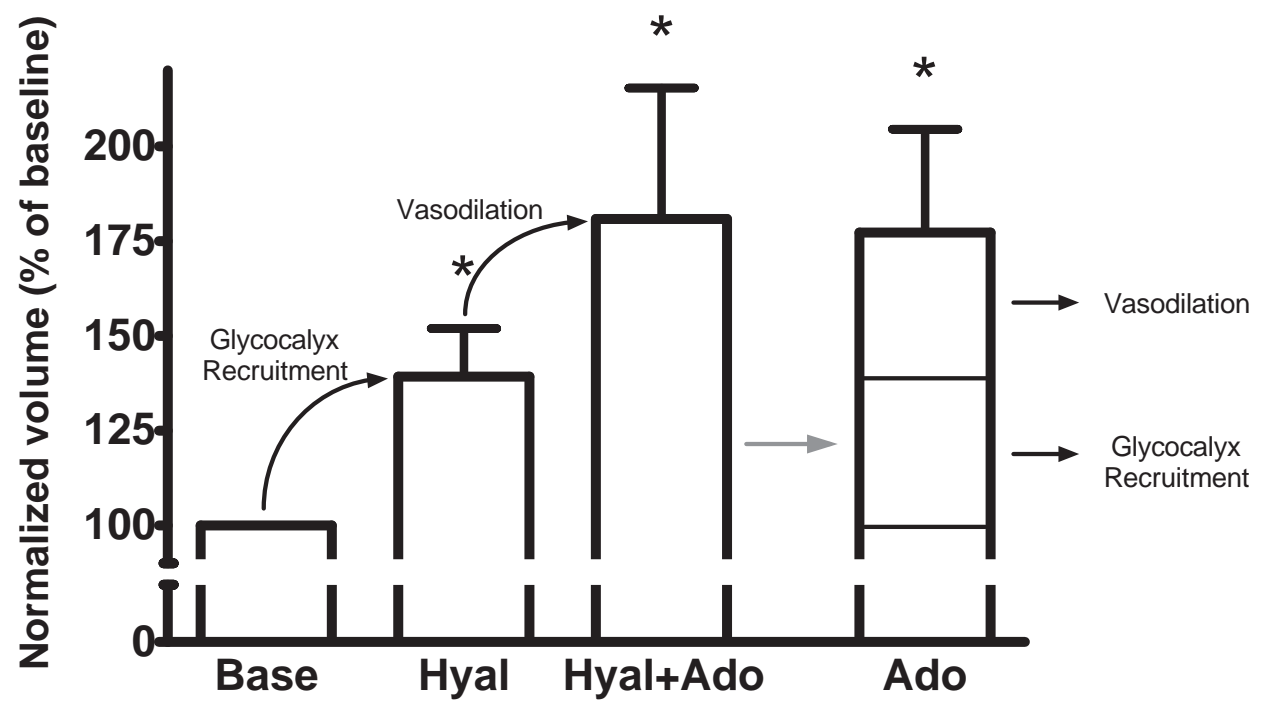

(b) Normalized to baseline coronary blood volumes 
red blood cells did not change upon infusion of adenosine $(4.5 \pm 0.5$ and $4.6 \pm 0.6$ sec for Dex-2000 and red blood cells, respectively).

The sum of red blood cell and Dex-2000 distribution volumes, figure 7.3, yielded total coronary blood volume during each condition. Total coronary blood volume at baseline was $18.9 \pm 1.1 \mathrm{ml}$ per 100 gram heart tissue and increased to $26.3 \pm 2.7 \mathrm{ml} / 100 \mathrm{gr}$ $(\mathrm{P}<0.05)$ after hyaluronidase treatment. Adenosine infusion after hyaluronidase resulted in coronary blood volume of $33.9 \pm 6.8 \mathrm{ml} / 100 \mathrm{gr}$. Coronary blood volume during adenosine was not different before and after hyaluronidase treatment (33.2 \pm $5.3 \mathrm{ml} / 100 \mathrm{gr}$ before hyaluronidase degradation of the glycocalyx).

To verify that the increase in coronary blood volume after hyaluronidase was not the result of incomplete recovery after adenosine, baseline coronary blood volume was measured after infusion of vehicle instead of hyaluronidase in three additional experiments. No difference in baseline blood volume was observed before and after vehicle administration $(14.4 \pm 4.1$ and $14.9 \pm 4.5 \mathrm{ml} / 100 \mathrm{gr}$, respectively; relative change: $104.1 \pm 8.3 \%)$, indicating that the effect of adenosine $(23.1 \pm 5.9 \mathrm{ml} / 100 \mathrm{gr}$ and 21.7 $\pm 6.5 \mathrm{ml} / 100 \mathrm{gr}$, not significant) on coronary blood volume was transient.

Adenosine infusion resulted in an increase in coronary conductance (figure 7.4) from a baseline of $2.1 \pm 0.2$ to $4.7 \pm 0.4 \mathrm{ml} / \mathrm{min} / 100 \mathrm{gr} / \mathrm{mmHg}(\mathrm{P}<0.05)$. After hyaluronidase treatment, baseline coronary conductance $(2.4 \pm 0.3 \mathrm{ml} / \mathrm{min} / 100 \mathrm{gr} / \mathrm{mmHg})$ and maximal adenosine-induced coronary conductance $(5.3 \pm 0.6 \mathrm{ml} / \mathrm{min} / 100 \mathrm{gr} / \mathrm{mmHg})$ were not changed compared to their corresponding measurements before hyaluronidase treatment. 




Figure 7.4 Coronary conductance at baseline, after hyaluronidase, and during maximal vasodilation with adenosine, before and after hyaluronidase. ${ }^{*} \mathrm{P}<0.05$ from baseline measurement before and after hyaluronidase.

\section{Discussion}

In the current study in anesthetized goats, we demonstrated that intracoronary hyaluronidase infusion increased baseline coronary blood volume by $40 \%$, see figure 7.3(b). Further increases in coronary blood volume of approximately $35 \%$ by adenosine on top of maximal glycocalyx porosity by hyaluronidase might then represent increases in vascular volume as a result of adenosine's well-known vasodilating capacity, suggesting that increases in coronary blood volume by increased glycocalyx porosity and vasodilation are of similar magnitude. Maximal coronary blood volume following administration of adenosine was identical with and without prior glycocalyx degradation by hyaluronidase, indicating that adenosine might increase glycocalyx porosity in a hyaluronidase like manner. In line with this possibility, we report that administration of adenosine resulted in a twofold increase in the concentration of plasma hyaluronan in the coronary effluent. Plasma hyaluronan levels during hyaluronidase 
still further increased compared with those during adenosine administration, indicating that the shedding of hyaluronan from the glycocalyx was less sensitive to adenosine compared with enzymatic degradation. Nevertheless, the adenosine-induced shedding of hyaluronan during adenosine appeared to result in similar loss of blood-excluding barrier properties of the glycocalyx compared with hyaluronidase treatment, suggesting that the remaining hyaluronan structures during adenosine do not constitute a serious glycocalyx barrier for the exclusion of flowing blood.

However, although available data demonstrate that enzymatic glycocalyx degradation greatly impairs the potential for adenosine to increase coronary blood volume, we report that adenosine's potential to increase coronary conductance was not affected by glycocalyx degradation, demonstrating that glycocalyx mediated modulation of microvascular blood volume has very little impact on coronary conductance during administration of adenosine and that assessment of coronary flow reserve capacity is therefore insufficient to detect glycocalyx damage and subsequent impairment of coronary blood volume reserve.

\section{Methodological considerations}

As a consequence of the instrumentation, atrial natriuretic peptide (ANP) might have been released into the coronary system and consequently induced shedding of the endothelial glycocalyx [11]. We assume that the instrumentation phase mainly might have contributed to a potential release of ANP in our experiments and that the levels were more or less constant during the successive coronary blood volume measurements. Furthermore, presence of heparin to prevent coagulation has been associated with impaired glycocalyx barrier properties in mice [150]. Both ANP release and heparin infusion might therefore have caused a reduced baseline coronary glycocalyx volume in our study. As a result, the recruited coronary glycocalyx volume during adenosine and after hyaluronidase might have been underestimated.

\section{Indicator-dilution-technique}

In the current study, we measured coronary blood volumes by applying the tracerdilution technique in open-thorax goat hearts using a perfusion-system. The perfusion system enables injection of tracers and control of perfusion pressure independent of 
Glycocalyx degradation uncouples coronary blood flow and volume regulation

cardiac function. Measuring distribution volumes in a beating heart in vivo makes it impossible to fulfill all requirements associated with the indicator-dilution-technique. Flow and volume in a beating heart are not stationary, for example. However, flow and volume variations at cardiac frequencies have been indicated to produce little error as long as the cycle length is less than a quarter of the dye curve passage time $[5,84]$. In our measurements, there were approximately 10 heart beats during the passage of the indicator from the left main to the great cardiac vein; therefore, we expect a minimal error due to fluctuation in flow and volume. On the other hand, the respiration frequency is much lower, and although the respiration frequency was held constant, 13-15 breaths per minute, it may have introduced an error in the measurement. To overcome the problem of contamination of the outflow by recirculating tracer, a local density random walk fit was used that was based on the curve up to $15 \%$ of the peak concentration on the descending slope of the outflow concentration and labeled red blood cell fraction curves [7]. In the current study we used a perfusion system with a relative large volume $(\sim 110 \mathrm{ml})$, delaying the moment recirculation started to interfere with the first pass outflow curve (with a minimum of 13.3 seconds at the highest flow measured in the current study $(495.1 \mathrm{ml} / \mathrm{min})$ ), and as a result, data up to a relative low percentage of the maximum peak value could be used. The fitting of the curves provided results with average correlation coefficients of $\mathrm{R}^{2}=0.968$ (standard deviation equal to 0.03 ) for the plasma tracer and $\mathrm{R}^{2}=0.973$ (standard deviation equal to 0.04 ) for the red blood cell tracer. To minimize and standardize the influence of injection on MTT, the tracers in this study were injected in approximately 1 second, always by the same person. Since we injected the tracers at the entrance of the left main coronary artery, complete mixture of the tracers with the flowing blood may not have occurred. However, our concentration outflow curves showed little to no noise (see figure 7.1), suggesting a well mixing of the tracers with the flowing blood.

\section{Tracers}

We used two tracers to obtain coronary blood volume measures, a red blood cell tracer and a plasma tracer. We used labeled red blood cells and flow cytometry for measuring red blood cell distribution volume [103]. The red blood cells, labeled the day before the experiment, were kept at $4^{\circ} \mathrm{C}$ until the experiment. No differences in size were found among labeled cells, normal unlabeled cells, and cells that were kept 
overnight at $4^{\circ} \mathrm{C}$ (data not shown). Furthermore, no effect in labeling efficiency was found between labeled cells that were kept overnight at $4^{\circ} \mathrm{C}$ and freshly collected and labeled cells. As a result, the distribution of labeled red blood cells can be considered representative to that of the normal, unlabeled, red blood cells in the body.

As intravascular plasma tracer we used FITC labeled dextrans with a molecular weight of $2000 \mathrm{kDa}$. Intravital microscopic studies have demonstrated the intraluminal disposition and significant exclusion by the glycocalyx of dextrans with a molecular weight of $70 \mathrm{kDa}$ and larger in microvessels of striated muscle [47,158], indicating their usefulness as intravascular plasma tracers. In those studies, a systemic bolus infusion (140 U [47] or $100 \mathrm{U}$ [12]) of hyaluronidase was shown to increase glycocalyx porosity for dextrans of 70 and $145 \mathrm{kDa}$, but not for the larger-size dextrans. We observed a $41 \pm 16 \%$ increase in Dex-2000 distribution volume without a change in coronary conductance after hyaluronidase, suggesting a robust increase in glycocalyx porosity for these dextrans. Moreover, RBC distribution volume increased $34 \pm 6 \%$, indicating that the increase in glycocalyx porosity was associated with an increase in functionally perfused blood volume. The present effect of hyaluronidase on large dextran distribution might be the result of the prolonged exposure of the coronary vascular bed to a higher dose of hyaluronidase. Thus, in the current study, hyaluronidase was administered as a continuous intracoronary infusion during 50 minutes at a much higher dose (170.000 U in total). Enzymatic glycocalyx degradation using heparinase and pronase was demonstrated in isolated coronary arterioles to result in a manifold increase in apparent vascular permeability for the plasma proteins $\alpha$-lactalbumin and albumin, which have a size of 14 and $65 \mathrm{kDa}$, respectively, [52]. We anticipate, however, that the first-pass outflow curve of the 30 fold larger $2000 \mathrm{kDa}$ dextrans measured after hyaluronidase treatment represented their intravascular distribution in circulating plasma. The relative increase in Dex-2000 distribution volume after hyalurondiase was not different from the relative increase in red blood cell volume, confirming that after hyaluronidase the distribution volume of Dex-2000 indeed likely represents the circulating plasma volume.

\section{Hemodynamics}

Our reported coronary baseline blood volume of $14-19 \mathrm{ml} / 100 \mathrm{gr}$ is in the high range of coronary blood volumes found in literature using the indicator dilution technique 
Glycocalyx degradation uncouples coronary blood flow and volume regulation

(from 7.3 up to $17.8 \mathrm{ml} / 100 \mathrm{gr}$ ) $[22,49,91,168]$. These relatively high baseline blood volumes coincide with relatively high baseline coronary blood flows in the current study, 190-250 ml/min/100gr (table 7.1). Whereas baseline coronary blood flows reported in anesthetized large animals in the literature are typically $\sim 100 \mathrm{ml} / \mathrm{min} / 100 \mathrm{gr}$ [32], the higher flows in the current study should be explained by a reduction in basal vasomotor tone of the resistance vessels due to the thorough instrumentation of the heart, including the use of a perfusion system, and the relatively high heart rates and perfusion pressures. Nevertheless, increases in coronary conductance during maximal adenosine were $\sim 2.3$ fold, illustrating that there was sufficient potential for vasodilation.

Induction of anesthesia and the very initial surgery (until opening of the chest) were associated with a large decrease in large vessel hematocrit. Hematocrit of blood taken the day before the experiment was $32.6 \pm 1.6 \%$, corresponding nicely with the normal value of $34 \%$ that is reported in goats [171], but decreased immediately after the start of surgery to $24 \pm 1.7 \%$. Subsequent surgery with open-thorax further decreased the hematocrit to $\sim 21 \%$, see table 7.1 , and this small decrease might be explained by the addition of $\sim 110 \mathrm{ml}$ of Gelofusine (B.Braun), used for filling the perfusion system. The $\sim 2 \%$ decrease in hematocrit during the following blood volume measurements are likely the result of the blood sampling ( $\sim 20 \mathrm{ml}$ per measurement) and subsequent replacement of lost blood with infusion fluid.

\section{Acute attenuation of glycocalyx barrier properties increases coronary blood volume}

The increase in coronary blood volume by adenosine found in this study, $\sim 75 \%$ of baseline volume, is consistent with the effect of this vasodilator in other studies, i.e. increases of $30 \%$ to $100 \%$ have been reported $[22,51,143,161]$ using different techniques; magnetic resonance imaging, measurements of oxygen content, X-ray contrast enhancement and myocardial radioactivity. Hyaluronidase induced degradation of the glycocalyx constituent hyaluronan increases the glycocalyx porosity. As a result, coronary blood volume increased with $\sim 40 \%$. Subsequent to the degradation of glycocalyx by hyaluronidase, the additional $35 \%$ increase in coronary blood volume by administration of adenosine is most likely due to the vasodilator effects of adenosine 
on the resistance vessels.

\section{Adenosine-induced blood volume recruitment}

Our data support the hypothesis that adenosine can increase microvascular blood volume by modulating the barrier properties of the endothelial glycocalyx and that this potential for recruitment is lost upon glycocalyx degradation [151,154], attenuating the effect of adenosine on coronary blood volume increases. Our observation is in line with the intravital microscopic studies in cremaster tissue by Desjardins \& Duling [29] who showed that whereas in control tissue, adenosine superfusion increased capillary tube hematocrit two- to threefold, adenosine did not increase the already elevated capillary tube hematocrit after heparinase treatment of the glycocalyx. Duling and coworkers $[29,74]$ were the first to suggest the possibility that the increase in capillary blood volume during adenosine administration could be the result of a reduction in glycocalyx exclusion volume, and, more recently, their group showed an increased porosity for $70 \mathrm{kDa}$ dextrans into the glycocalyx domain [110]. Recent observations from our group in mouse cremaster muscle showed that, similar to the effect of adenosine, the vasodilators bradykinin and sodium nitroprusside also increased capillary tube hematocrit and that the increase was paralleled by an increased porosity of the glycocalyx for $70 \mathrm{kDa}$ dextrans [152]. These effects were absent in hypercholesterolemic mice, which had an elevated capillary tube hematocrit and glycocalyx porosity under baseline conditions already, indicating glycocalyx degradation. Whereas these previous studies assessed changes in tube hematocrit and glycocalyx porosity in microvessels of cremaster tissue, we extended these observations to the coronary circulation of a large animal and determined the contribution of the glycocalyx to baseline and adenosine-induced total coronary blood volume in the heart.

\section{Measurement of coronary flow reserve using adenosine}

Adenosine is the preferred vasodilator in the clinic for assessment of the relevance of coronary artery stenosis and perfusion abnormalities. During adenosine administration in the current study, there was an equal increase in flow and coronary conductance before and after hyaluronidase. Although hyaluronidase treatment has been 
Glycocalyx degradation uncouples coronary blood flow and volume regulation

associated with a reduction in shear-induced endothelium-derived nitric oxide (NO) release $[90,107]$, the equal increase in coronary conductance during adenosine infusion before and after hyaluronidase treatment implies that a potential hyaluronidaseassociated depression of NO production did not affect the flow increase during adenosine. The coronary flow reserve appeared, therefore, unlike the volume that could be recruited, not influenced by enzymatic treatment of the glycocalyx, demonstrating that measurement of coronary flow reserve is insufficient to detect impairment of coronary blood volume recruitment in conditions of damaged glycocalyx.

The mechanism of coronary blood volume regulation appears to be, to some extent, uncoupled from that of blood flow regulation. It can be anticipated that the increase in coronary blood flow during adenosine is primarily a result of relaxation of the resistance vessels, whereas the coronary blood volume increase during adenosine is due to both recruitment of glycocalyx volume and vasodilation. Assuming complete removal of the glycocalyx after hyaluronidase, the remaining volume increase during adenosine infusion after enzymatic glycocalyx treatment (i.e., from $26.3 \pm 2.7$ to 33.9 $\pm 6.8 \mathrm{ml} / 100 \mathrm{gr}$ ) will mainly represent the volume increase due to vasodilation only.

\section{Schematic representation}

The endothelial glycocalyx is situated at the luminal side of endothelial cells throughout the entire vascular bed, and its thickness has been estimated to vary between different vessel types, from $0.2-0.9 \mu \mathrm{m}$ in capillaries [47,100,103,110,111,121,152,157,158], to $2-3 \mu \mathrm{m}$ in small arteries with a diameter of $\sim 150 \mu \mathrm{m}$ [144], to $4-5 \mu \mathrm{m}$ in carotid arteries [83]. Since by far the majority of the endothelial surface is in the microcirculation (of which $\sim 90 \%$ is capillaries [64]), these numbers indicate that the glycocalyx occupies a large part of the anatomic vascular volume particularly in the microcirculation. Therefore, we suggest that the reported effects on glycocalyx recruitment are to a large extent occurring in the microvascular compartment, particularly in the capillaries. In figure 7.5, we have summarized schematically the hypothesized effects of hyaluronidase and adenosine on capillary (top panel) and resistance vessel (bottom) blood volume and glycocalyx porosity. At baseline there is a blood-excluding glycocalyx present on the endothelial cells reducing functionally perfused capillary volume for flowing blood, as shown with intravital microscopy [47,100,110,111,121,152,157,158]. During adenosine infusion, recruitment of glycocalyx volume causes a robust increase 
in microvascular blood volume as reflected by the increase in capillary red blood cell content (and tube hematocrit), whereas the concurrent dilation of resistance vessels accounts for the coronary conductance increase as well as part of the increase in microvascular blood volume. Hyaluronidase induces a loss of blood-excluding glycocalyx volume, causing a significant increase in microvascular perfused volume at baseline. The increase in coronary blood volume during adenosine after hyaluronidase, only by vasodilation of the resistance vessels, is, as a result, reduced compared with the increase in coronary blood volume during adenosine with an intact glycocalyx. The impaired potential for glycocalyx volume recruitment can occur without a change in adenosine-induced increases in coronary conductance because of an unaltered dilator response of the resistance vessels to adenosine.

\section{Physiological and clinical relevance}

We found under control conditions, i.e., in the presence of an intact glycocalyx, that the transit time for red blood cells and dextrans through the coronary system was not reduced during adenosine despite a 1.7 fold increase in blood flow due to a concomitant increase in coronary blood volume. Consistent with this finding, direct observations of epicardial coronary capillary hemodynamics in the canine heart by Kiyooka et al. [73] showed that a 4.2 fold increase in blood flow with adenosine was associated with only modest increases in capillary red blood cell velocity in the epicardial capillaries. After hyaluronidase treatment of the glycocalyx, red blood cell transit time decreased during adenosine infusion compared with baseline transit times. Loss of glycocalyx volume recruitment capacity therefore could possibly impair the available time for red blood cells to offload oxygen when blood flow is increased to meet increases in metabolic demand. In line with this, we found in the current study a $20-30 \%$ decrease in myocardial oxygen consumption during administration of adenosine after glycocalyx degradation by hyaluronidase. Since in addition to regulation of blood transit time during conditions of increased blood flow, recruitment of glycocalyx volume in the capillaries also augments the surface area for exchange in these vessels, agonistinduced modulation of glycocalyx volume might provide a mechanism for the heart to match exchange capacity to increases in flow, e.g., during exercise.

Angina during exercise has been generally related to a diseased macrocirculation, for example, the presence of flow-limiting stenosis in a large coronary artery. A consid- 

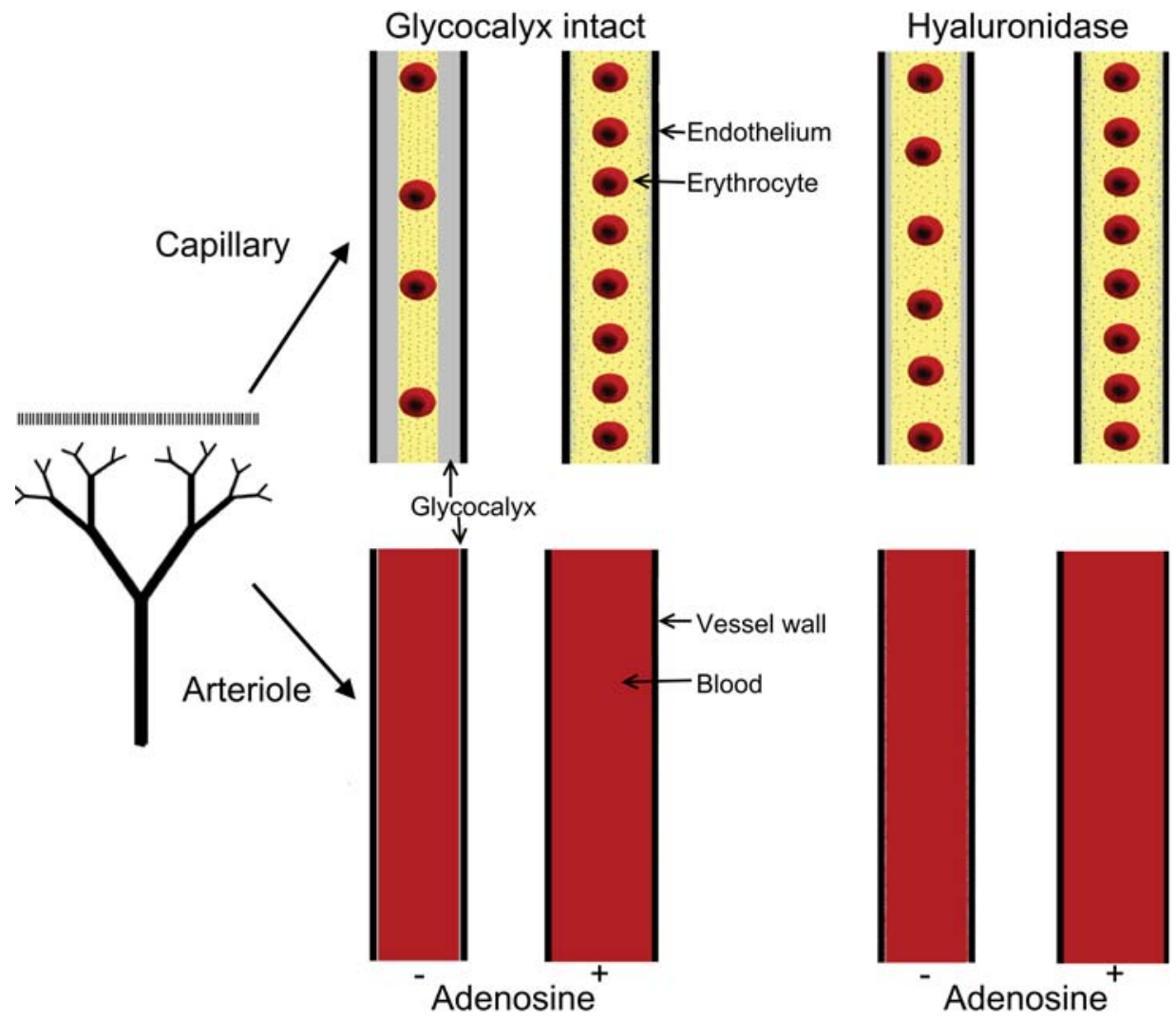

Figure 7.5 Schematic representation of presumed distribution of red blood cells and large anionic dextrans in coronary capillaries (top) and arterioles (bottom). The blood excluding glycocalyx is displayed in gray, the plasma distribution in yellow, the red blood cells in red, and the dextrans are represented as small dots. A) Baseline; intact glycocalyx reduces functionally perfused capillary volume, basal vasomotor tone reduces arteriolar blood volume. B) During adenosine; recruitment of glycocalyx volume in addition to dilation of arterioles results in a maximal increase in perfused microvascular volume. C) After hyaluronidase; impaired glycocalyx exclusion increases baseline blood volume, particularly in the capillaries. D) Adenosine after hyaluronidase results again in a maximal perfused microvascular volume yet a diminished blood volume recruitment due to the increase in baseline volume as a result of glycocalyx loss.

erable number of patients with symptoms of chest pain during exercise, however, do not show a prominent stenosis in their coronary arteries or coronary artery disease at all [62]. Furthermore, a large number of the patients with a detectable lesion have a hemodynamically non-significant intermediate coronary artery stenosis, and these 
patients do not benefit from a percutaneous coronary intervention [109]. Rather, in all these cases, the angina may relate to coronary microvascular dysfunction [13], of which loss of glycocalyx may be an early trigger $[10,150,151]$. The current findings suggest that, rather than coronary flow reserve, the measurement of coronary blood volume recruitment may discriminate loss of glycocalyx. As such, MRI [59,161] or contrast-enhanced ultrasound using microbubbles $[69,70]$ may be appropriate noninvasive methods for early detection of coronary vulnerability due to glycocalyx loss in patients with angina but without overt coronary artery disease.

\section{Acknowledgments}

The authors would like to thank Marion Kuiper, Anniek Lampert, Arne van Hunnik, Bart Eskens and Hanneke Cobelens for their assistance. 


\section{Hyaluronidase-induced}

recruitment of glycocalyx volume increases myocardial oxygen exchange in the goat heart

The contents of this chapter are based on Judith Brands, Hans Vink and Jurgen WGE Van Teeffelen Recruitment of glycocalyx volume increases myocardial oxygen exchange in the goat heart 


\section{Abstract}

Aim A relatively thick endothelial glycocalyx forms the true interface between blood and the vascular wall, and thereby limits microvascular volume available for perfusion and exchange. In a previous study we have shown that hyaluronidase treatment increases coronary microvascular blood volume. We now hypothesize that hyaluronidaseinduced recruitment of glycocalyx volume increases microvascular oxygen exchange. Method In the current study the effect of hyaluronidase on myocardial oxygen exchange was assessed in goats, of which the hearts were paced $(\mathrm{N}=8)$. Blood samples were collected simultaneously from the great cardiac vein and aorta at heart rates of 80,110, 140 and 170 beats per minute, and coronary blood flow was measured continuously. The measurement was done at baseline and repeated after intracoronary hyaluronidase $(\mathrm{N}=5)$ or phosphate buffered saline (vehicle; $N=3$ ) treatment. Results Hyaluronidase treatment increased the myocardial arteriovenous oxygen difference $\left(\mathrm{AV}_{o x}\right)$ significantly from $7.0 \pm 0.2$ to $8.3 \pm 0.3 \mathrm{ml} / 100 \mathrm{ml}$, while vehicle administration had no effect on $\mathrm{AV}_{o x}(7.7 \pm 0.2$ and $8.0 \pm 0.1 \mathrm{ml} / 100 \mathrm{ml}$, respectively). Coronary blood flow decreased significantly in time in both groups (76.4 \pm 4.8 at baseline and $63.1 \pm 4.1 \mathrm{ml} / \mathrm{min} / 100 \mathrm{gr}$ after vehicle; $74.7 \pm 8.6$ at baseline and $54.9 \pm 4.7 \mathrm{ml} / \mathrm{min} / 100 \mathrm{gr}$ after hyaluronidase). While in the control group, myocardial oxygen consumption $\left(\mathrm{MVO}_{2}\right)$ was decreased by $\sim 12 \%$, in line with a $\sim 14 \%$ decrease in rate-pressure-product (RPP) in these animals, $\mathrm{MVO}_{2}$ and RPP were unaltered in the hyaluronidase treated group. Conclusion These data indicate that hyaluronidase-induced recruitment of coronary endothelial glycocalyx volume is associated with an increase in myocardial oxygen extraction. Glycocalyx recruitment may, therefore, constitute a mechanism by which the heart can adapt its oxygen exchange to its needs.

\section{Introduction}

The heart has been documented to achieve high coronary arteriovenous oxygen extraction, about $60-70 \%$ at rest [32]. As a result of the high extraction rate, it is the general opinion that a four- to fivefold increase in myocardial oxygen consumption, for instance during exercise, is mainly matched by comparable increases in coronary 
Glycocalyx limits myocardial oxygen exchange in the goat heart

blood flow [30,32], and, to a minor extent, by an increase in extraction (arteriovenous oxygen difference) $[44,71,72,97,160]$. An increase in arteriovenous oxygen difference can be brought about by changes in metabolites [97], an increase in hematocrit [71], or an augmentation of capillary surface area. Capillary surface area can be enlarged by increasing the number of capillaries that are being perfused $[19,45,94]$ and by increasing perfused volume within a capillary, either by recruitment of endothelial glycocalyx $[29,47,74,152]$ or a pressure-induced increase in capillary diameter [9].

In the heart a substantial part of the microvascular volume has been suggested not to be entirely perfused with flowing blood under baseline conditions, but to be filled with a blood excluding endothelial glycocalyx [154]. Using the indicator dilution technique we recently showed in goat hearts that after hyaluronidase treatment of the glycocalyx in the coronary circulation, the coronary vascular blood volume was increased with $39 \pm 13 \%$ compared to the volume before enzyme treatment, chapter 7 . The relative thickness of the endothelial glycocalyx has been demonstrated to vary between different vessel types $[47,83,100,103,110,111,121,144,152,157,158]$. In small vessels, in particular capillaries, the relative amount of vascular volume occupied with glycocalyx is the largest. Since by far the majority of the endothelial surface in the heart is in the microcirculation (of which $\sim 90 \%$ is capillaries [64]), these numbers indicate that the glycocalyx occupies a large part of the anatomic vascular volume particularly in the coronary microcirculation.

We hypothesize that an increase in perfused microvascular volume by recruitment of endothelial glycocalyx volume increases oxygen exchange in the heart. In anesthetized goats, hearts were paced and arterial and coronary venous blood samples were taken simultaneously at baseline (intact glycocalyx) and after hyaluronidase treatment (degraded glycocalyx) or phosphate buffered saline administration (control). Compared to baseline, there was after hyaluronidase an increase in arteriovenous oxygen difference, and maintenance of myocardial oxygen consumption despite a reduction in coronary flow in time. Therefore, it appears that under baseline conditions myocardial oxygen exchange may be limited by the presence of the coronary endothelial glycocalyx. 


\section{Methods}

\section{General Surgery and Anesthesia}

All procedures and protocols were approved by the animal care and use committee of Maastricht University (DECno. 2007-195). Studies were conducted in accordance with the National Institutes of Health Guide for the Care and Use of Laboratory Animals. Experiments were performed on female adult goats of $46-73 \mathrm{~kg}(\mathrm{~N}=8)$. At the beginning of an experiment, the goats were given Nesdonal (20mg/kg, i.v.). Next, goats were intubated and ventilated with a 1:2 $\mathrm{O}_{2}$ :air mixture and anesthesia was maintained by intravenous administration of Fentanyl $(6 \mu \mathrm{g} / \mathrm{kg} / \mathrm{h})$, Dormicum $(0.8$ $\mathrm{mg} / \mathrm{kg} / \mathrm{h})$ and Pavulon $(0.3 \mathrm{mg} / \mathrm{kg} / \mathrm{h})$. Depth of anesthesia was adjusted according to stability of femoral artery blood pressure $\left(\mathrm{P}_{f e m}\right)$ and heart rate $(\mathrm{HR})$. When necessary, ventilation was adjusted to maintain oxygen and $\mathrm{CO}_{2}$ pressures within physiological limits, and sodium bicarbonate was administered to avoid acidosis.

A pace-electrode (Capturefix Novus 5076, Medtronic) was placed in the right ventricle via the jugular vein. Hereafter, the AV-node was ablated via the saphenous vein (RF Marinr@ (MC) series, Medtronic) using radiofrequency waves. Immediately after ablation the heart was paced at 80 beats per minute (BPM) and the ablation catheter withdrawn from the right ventricle. A cathether was placed via a femoral artery into the aorta to measure aortic blood pressure $\left(\mathrm{P}_{a o}\right)$. Subsequently, a catheter was placed via the carotid artery in the left ventricle to measure blood pressure $\left(\mathrm{P}_{l v}\right)$. Hereafter, a left thoracotomy was performed and the great cardiac vein was cannulated via the azygos vein to obtain coronary venous blood samples. A flowprobe (Transonic flowprobe, Transonic Systems Inc) was placed around the left main coronary artery (7 goats) or left circumflex artery (1 goat of the hyaluronidase group) to measure coronary blood flow $\left(\mathrm{Q}_{\text {cor }}\right)$. Next, an epicardial electrode was placed on the left atrium for atrial pacing. The atrium was paced at a frequency twice that of the ventricle with the first stimulation $80 \mathrm{~ms}$ after the ventricle was paced (to prevent simultaneous contraction of atrium and ventricle) and the second stimulation exactly halfway two stimulations of the ventricle. After placing all catheters, electrodes and flow probe the thorax was closed again and heparin was given $(5000 \mathrm{IE} / \mathrm{h})$.

$\mathrm{P}_{f e m}, \mathrm{P}_{a o}, \mathrm{P}_{l v}, \mathrm{P}_{l v, s y s}$ (defined as the maximal $\mathrm{P}_{l v}$ per beat), $\mathrm{dP}_{l v} / \mathrm{dt}, \mathrm{Q}_{c o r}$ and $\mathrm{HR}$ (determined from $\mathrm{P}_{l v}$ ) were stored for off-line analysis (IDEEQ $250 \mathrm{~Hz}$, IDEE). At 
Glycocalyx limits myocardial oxygen exchange in the goat heart

the end of the experimental procedures the thorax was reopened and a battery placed on the heart to induce ventricular fibrillation. The heart was taken out and total heart weight as well as the weight of the perfused area was measured. Perfusion area was determined by injection of white paint into the left main coronary artery, and dissection and weighing of the demarcated area.

\section{Experimental protocol}

After surgery, the preparation was allowed to equilibrate for 30 minutes. In each animal arterial (aorta) and coronary venous (great cardiac vein) blood samples (0.7 $\mathrm{ml}$ ) were collected simultaneously at a heart rate of 80 beats per minute (BPM) and approximately 60 seconds after an increase in heart rate to 110, 140 and 170 BPM, respectively. Heart rate was increased by $30 \mathrm{BPM}$ every $\sim 5$ minutes and within the 60 seconds after a heart rate step (before the blood samples were taken) blood pressure $\left(\mathrm{P}_{l v}\right.$ and $\left.\mathrm{P}_{a o}\right)$ and flow $\left(\mathrm{Q}_{c o r}\right)$ reached new steady state values. Samples were taken at different heart rates to study whether the relation between myocardial oxygen demand versus oxygen supply and exchange was altered after hyaluronidase. Collected blood samples were analyzed for blood gas $\left(\mathrm{pO}_{2}\right.$ and $\left.\mathrm{pCO}_{2}\right)$ and oximetry (hemoglobin and oxygen saturation) values using ABL 510 (Radiometer, Copenhagen). After the blood samples were taken at 170 BPM the heart rate was reduced to 80 BPM again and an amplatz left catheter (AL3 guiding catheter, Cordis) was placed via a femoral artery at the origin of the left main coronary artery using X-ray. In $\mathrm{N}=5$ animals an intracoronary infusion of $100 \mathrm{ml}$ hyaluronidase was started at $120 \mathrm{ml} / \mathrm{h}$ (2100 units/ml phosphate buffered saline, type IV-S, Sigma-Aldrich; intracoronary). In $\mathrm{N}=3$ animals $100 \mathrm{ml}$ of phosphate buffered saline (vehicle) was used as control measurement. The placement of the catheter was verified midway and at end of the infusion period. Following 50 minutes of infusion, the pacing protocol, as described above, was repeated.

\section{Data analysis}

Based on the blood gas and oximetry values of the simultaneous taken arterial and coronary venous blood samples the myocardial arteriovenous oxygen difference $\left(\mathrm{AV}_{o x}\right)$ and myocardial oxygen consumption $\left(\mathrm{MVO}_{2}\right)$ were determined. The myocardial arte- 
riovenous oxygen difference was defined as

$$
A V_{o x}=c O_{\text {art }}-c O_{v e n}
$$

in which

$$
\begin{gathered}
c O_{\text {art }}=\left(1.34 \times \mathrm{Hb}_{\text {art }} \times \mathrm{SatO}_{2, \text { art }} \times 0.01+0.003 \times p O_{2, a r t}\right) \\
c O_{\text {ven }}=\left(1.34 \times \mathrm{Hb}_{\text {ven }} \times \mathrm{SatO}_{2, \text { ven }} \times 0.01+0.003 \times p O_{2, \text { ven }}\right)
\end{gathered}
$$

and $\mathrm{Hb}$ is the plasma hemoglobin in gr/dL, $\mathrm{SatO}_{2}$ is the oxygen saturation in $\%, \mathrm{pO}_{2}$ is the oxygen pressure in $\mathrm{mmHg}$, and 0.003 is the amount of $\mathrm{O}_{2}$ in milliliters dissolved per $100 \mathrm{ml}$ plasma per $\mathrm{mmHg}$. Myocardial oxygen consumption was defined as the arteriovenous oxygen difference times the coronary blood flow (equation 8.3).

$$
M V O_{2}=A V_{o x} \times Q_{c o r}
$$

The average blood pressure and blood flow at each heart rate was determined during the first 15 seconds after the blood samples were taken. The effect of vehicle/hyaluronidase administration on blood pressure and coronary blood flow, $\mathrm{dP}_{l v} / \mathrm{dt}$, rate-pressure-product $(\mathrm{RPP}=\mathrm{HR} \times$ systolic left ventricle blood pressure $), \mathrm{AV}_{\text {ox }}$ and $\mathrm{MVO}_{2}$ was tested using a t-test, and the effect of $\mathrm{HR}$ was tested by linear regression after normalization to the value measured at 80 BPM. The relation between myocardial oxygen consumption, RPP and $\mathrm{AV}_{o x}$, was tested by means of the two-tailed Pearson correlation test. Results were considered statistically significant with $\mathrm{P} \leq 0.05$. Summary data are reported as means \pm SEM.

Table 8.1 Hemodynamic parameters across all heart rates at baseline and after vehicle (control group, $\mathrm{N}=3$ ) or hyaluronidase $(\mathrm{N}=5)$ administration

\begin{tabular}{|l|c|c|c|c|}
\hline \multirow{2}{*}{} & \multicolumn{2}{|l|}{ Vehicle } & \multicolumn{2}{l|}{ Hyaluronidase } \\
\cline { 2 - 5 } & Baseline & Intervention & Baseline & Intervention \\
\hline \hline $\mathrm{P}_{a o}(\mathrm{mmHg})$ & $92.7 \pm 6.6$ & $76.5 \pm 2.7^{*}$ & $103.3 \pm 4.6$ & $100.5 \pm 2.8$ \\
$\mathrm{P}_{\text {lv }, \text { sys }}(\mathrm{mmHg})$ & $106.7 \pm 5.6$ & $88.6 \pm 2.2^{*}$ & $119.2 \pm 4.8$ & $113.5 \pm 3.2$ \\
$\mathrm{dP}_{l v} / \mathrm{dt}(\mathrm{mmHg} / \mathrm{sec})$ & $2936 \pm 326$ & $2007 \pm 178^{*}$ & $3399 \pm 471$ & $2396 \pm 180^{*}$ \\
$\mathrm{RPP}(\mathrm{mmHg} / \mathrm{min})$ & $7175 \pm 829$ & $5858 \pm 499^{*}$ & $9248 \pm 664$ & $8700 \pm 585$ \\
\hline
\end{tabular}

Values are means $\pm S E M,{ }^{*}$ significant from corresponding baseline measurement $(P<0.05)$. 


\section{Results}

Baseline hemodynamic parameters are presented in table 8.1. After vehicle administration there was a significant reduction in blood pressure and $\mathrm{dP}_{l v} / \mathrm{dt}$ compared to baseline. After hyaluronidase there was also a reduction in $\mathrm{dP}_{l v} / \mathrm{dt}(\mathrm{P}<0.05)$, however, blood pressure was unaffected. As a result, the RPP across all heart rates



(a) Vehicle

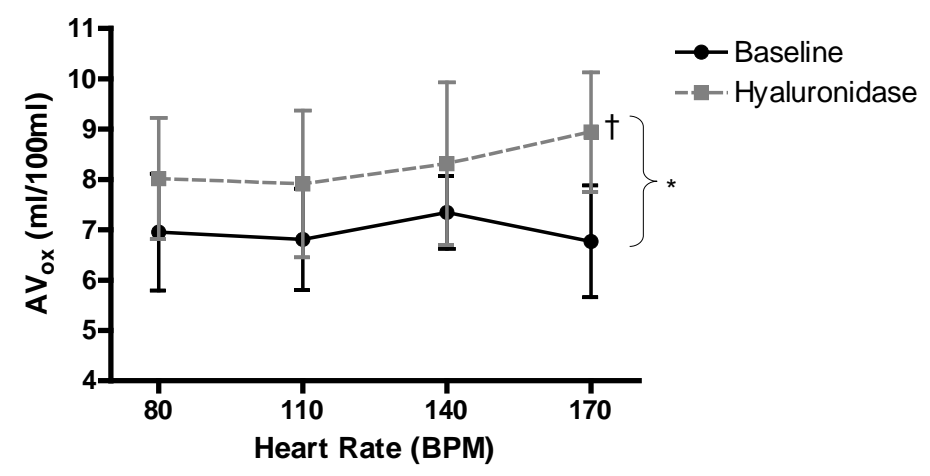

(b) Hyaluronidase

Figure 8.1 Arteriovenous oxygen difference $\left(\mathrm{AV}_{o x}\right)$ before (black) and after (gray) vehicle (a) and hyaluronidase (b) administration. After hyaluronidase there was a significant increase in $\mathrm{AV}_{\text {ox }}$ across all heart rates compared to the measurement before the intervention (120.9 $\pm 5.4 \%$ compared to baseline measurement, $\left.{ }^{*} \mathrm{P}<0.05\right)$. After hyaluronidase there is an increase in $\mathrm{AV}_{o x}$ with increasing heart rate (after normalization of the data to the value at $80 \mathrm{BPM}$ ), ${ }^{\dagger} \mathrm{P}<0.05$. 
was significantly decreased after vehicle administration but remained unaltered after hyaluronidase administration compared to its corresponding baseline measurement.

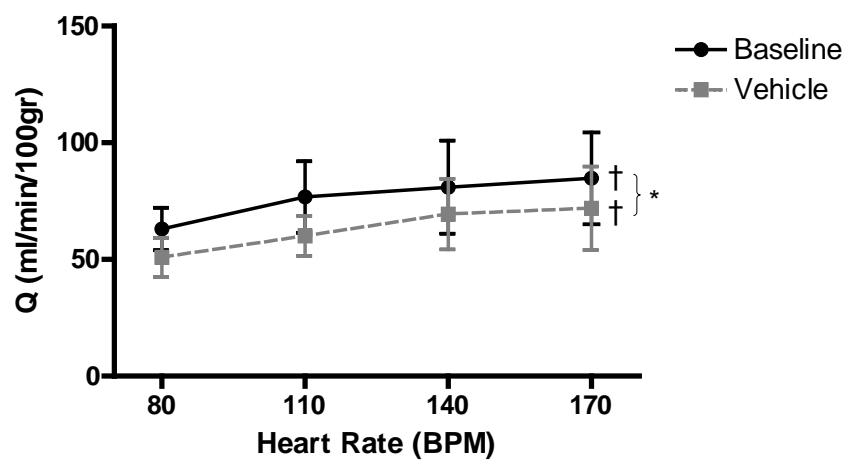

(a) Vehicle

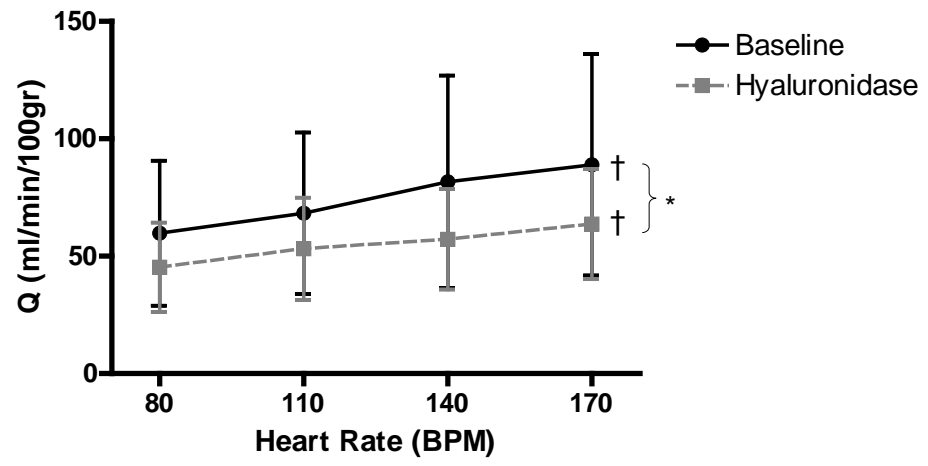

(b) Hyaluronidase

Figure 8.2 Coronary blood flow per 100 gram heart muscle tissue before (black) and after (gray) vehicle (a) and hyaluronidase (b) administration. After vehicle and hyaluronidase administration there was a significant reduction in coronary blood flow compared to baseline. After vehicle infusion the coronary blood flow across all heart rates was reduced to $83.7 \pm 4.7 \%$ of the flow measured at baseline $(* \mathrm{P}<0.05)$. Coronary blood flow across all heart rates after hyaluronidase was reduced to $80.3 \pm 5.1 \%$ of the flow measured at baseline $(* \mathrm{P}<0.05)$. The effect of hyaluronidase on the coronary blood flow was not significant different from the effect of vehicle administration. Coronary blood flow increased with increasing heart rate for all measurements (after normalization of the data to the value at $80 \mathrm{BPM}),{ }^{\dagger} \mathrm{P}<0.05$.

Coronary blood flow, myocardial oxygen consumption and RPP all increased with increasing heart rate $(\mathrm{P}<0.05$, see figure 8.2 and 8.3). The arteriovenous oxygen dif- 
ference $\left(\mathrm{AV}_{o x}\right)$ remained constant with increasing heart rate; it was only significantly influenced by heart rate after hyaluronidase was administered, figure 8.1.

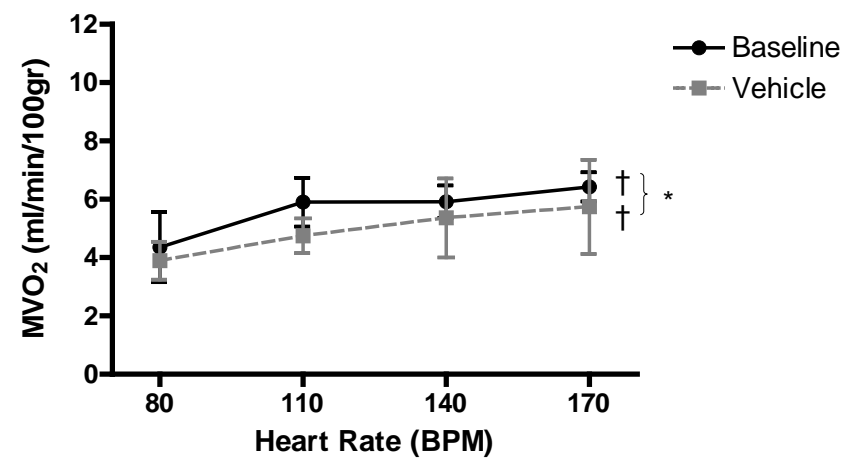

(a) Vehicle

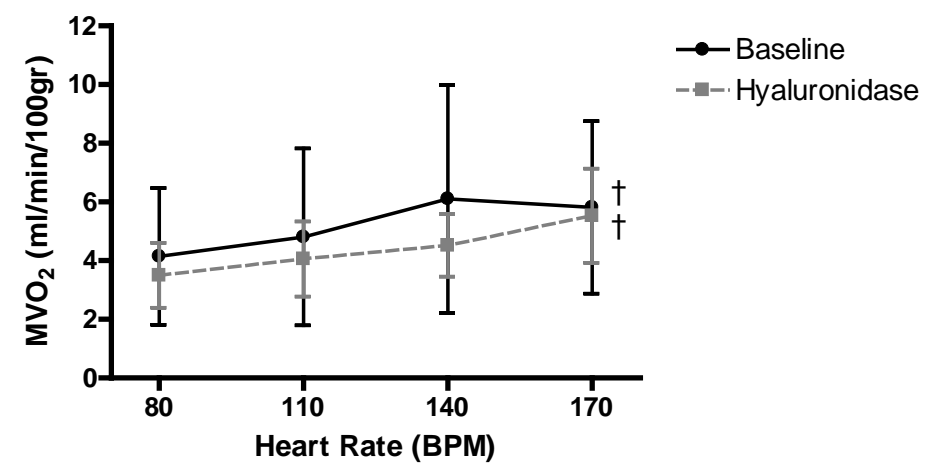

(b) Hyaluronidase

Figure 8.3 Myocardial oxygen consumption $\left(\mathrm{MVO}_{2}\right)$ before (black) and after (gray) vehicle (a) and hyaluronidase (b) administration. The $\mathrm{MVO}_{2}$ across all heart rates was $87.9 \pm 4.1 \%$ of baseline after vehicle administration $\left({ }^{*} \mathrm{P}<0.05\right)$. After hyaluronidase the $\mathrm{MVO}_{2}$ was not different from the myocardial oxygen consumption at baseline. Myocardial oxygen consumption increased with increasing heart rate for all measurements (after normalization of the data to the value at 80 BPM), † $\mathrm{P}<0.05$.

The $\mathrm{AV}_{\text {ox }}$, figure 8.1, was in the control group not influenced by the infusion of the vehicle. Before vehicle infusion the difference in oxygen content across all heart rates was $7.7 \pm 0.2 \mathrm{ml} / 100 \mathrm{ml}$, which is not different from $8.0 \pm 0.1 \mathrm{ml} / 100 \mathrm{ml}$ measured after administration of the vehicle. For the hyaluronidase group of animals the $\mathrm{AV}_{o x}$ 
at baseline across heart rates was $7.0 \pm 0.1 \mathrm{ml} / 100 \mathrm{ml}$ and increased significantly after hyaluronidase infusion to $8.3 \pm 0.2 \mathrm{ml} / 100 \mathrm{ml}(\mathrm{P}<0.05)$.

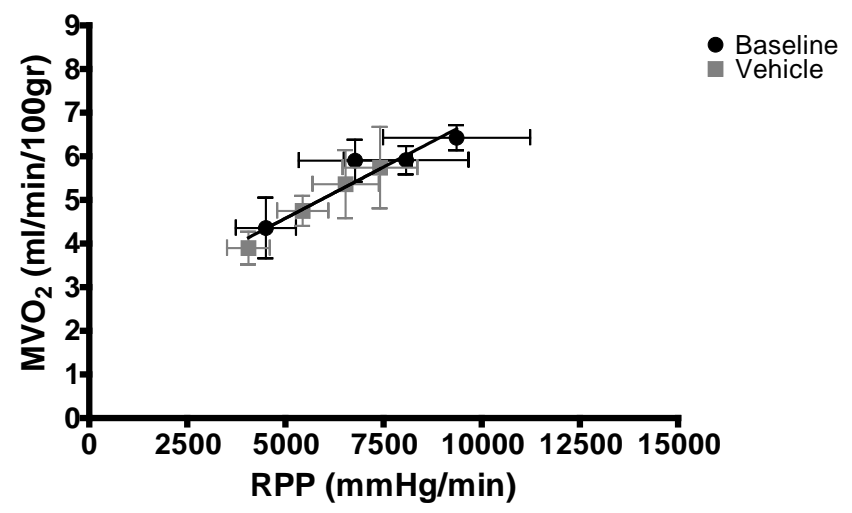

(a) Vehicle



(b) Hyaluronidase

Figure 8.4 Rate-pressure-product (RPP) versus myocardial oxygen consumption $\left(\mathrm{MVO}_{2}\right)$ before (black) and after (gray) vehicle (a) and hyaluronidase (b) administration.

Comparing before with after vehicle infusion there was a reduction in flow $(\mathrm{P}<0.05)$ after vehicle administration $(83.7 \pm 4.7 \%$ of baseline across heart rates). The flow versus heart rate after the vehicle intervention (figure 8.2a) was shifted parallel downward compared to the data before vehicle was given. Similar for hyaluronidase, after the 
administration of the enzyme coronary blood flow was significantly decreased $(80.3 \pm$ $5.1 \%$ of baseline across heart rates).



Baseline

Vehicle

(a) Vehicle

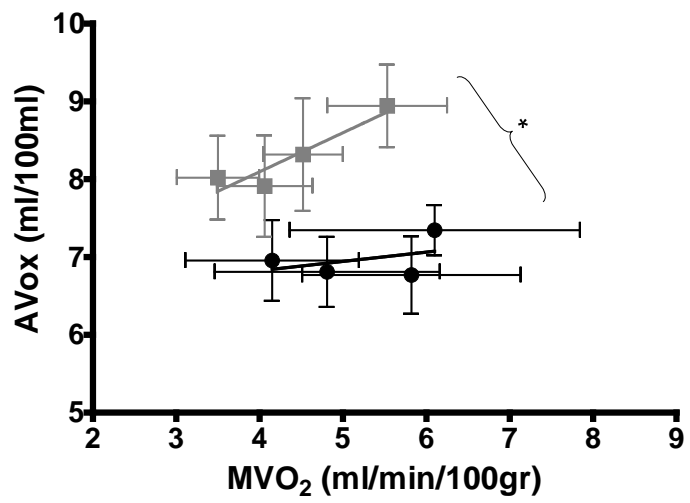

- Baseline

- Hyaluronidase

(b) Hyaluronidase

Figure 8.5 Arteriovenous oxygen difference $\left(\mathrm{AV}_{o x}\right)$ versus myocardial oxygen consumption $\left(\mathrm{MVO}_{2}\right)$ before (black) and after (gray) vehicle (a) and hyaluronidase (b) administration. The relation between $\mathrm{AV}_{\text {ox }}$ with $\mathrm{MVO}_{2}$ after hyaluronidase was significantly different from corresponding baseline measurements $\left({ }^{*} \mathrm{P} \leq 0.05\right)$.

After vehicle infusion the $\mathrm{MVO}_{2}$ across heart rates was reduced to $87.9 \pm 4.1 \%$ of the myocardial oxygen demand at baseline across heart rates (figure 8.3). In contrast, the $\mathrm{MVO}_{2}$ after hyaluronidase administration was unaltered compared to the baseline 
measurement, $95.9 \pm 6.8 \%$ of baseline across heart rates (N.S.).

There was a significant correlation between $\mathrm{MVO}_{2}$ and rate-pressure-product, except for baseline before hyaluronidase. Comparing baseline measurements with the data after the infusion of hyaluronidase or the vehicle, the relation between oxygen consumption and RPP was unaltered, as depicted in figure 8.4. No statistical relevant correlation was found between $\mathrm{AV}_{\text {ox }}$ and $\mathrm{MVO}_{2}$ for any of the measurements (figure 8.5a en 8.5b), indicating that the increase in $\mathrm{MVO}_{2}$ with an increase in heart rate was primarily the result of an increase in blood flow and not in extraction. However, as a result of the increase in $\mathrm{AV}_{o x}$ across all heart rates after hyaluronidase, a difference was found between the regression lines for the data before versus after hyaluronidase administration $(\mathrm{P}<0.05)$, see figure $8.5 \mathrm{~b}$. This difference was not observed for regression lines before versus after vehicle infusion (figure 8.5a).

\section{Discussion}

In the current study in anesthetized goats we demonstrated that hyaluronidase treatment of the coronary circulation resulted in an increased myocardial extraction of oxygen. The data in this study suggest that presumed recruitment of coronary vascular volume by endothelial glycocalyx degradation might contribute to increases in oxygen exchange of the heart. In general, recruitment of glycocalyx volume may enhance exchange of oxygen and other nutrients during conditions of increased metabolic demand of the heart.

\section{Methodological considerations}

Hyaluronidase was given by directing a catheter at the entrance of the left main using $\mathrm{X}$-ray. Some of the hyaluronidase given might not have entered the coronary vasculature but might have spilled out into the aorta. Systemic effects of hyaluronidase that have been reported include increased vascular leakage, impairment of shear dependent NO production, and a decrease in number of perfused capillaries [12,90,142]. These effects are expected to be mild because of the considerable dilution of hyaluronidase in the circulating blood.

A time dependent deterioration of the condition of the hearts, as reflected by the 
decrease in $\mathrm{dP}_{l v} / \mathrm{dt}$ (table 8.1), can be expected due to factors like anesthesia, artificial respiration and instrumentation. In the control experiments (phosphate buffered saline without the enzyme hyaluronidase), there was also a reduction in systolic left ventricle blood pressure and aortic blood pressure. In contrast, no drop in blood pressure was observed in the hyaluronidase experiments. The difference in aortic blood pressure between the two groups (control versus hyaluronidase) indicates differences in total peripheral resistance and/or cardiac output. Based on the measured parameters we cannot determine what causes the difference in blood pressure, but, as explained in the previous paragraph, we cannot rule out that a reduction in NO bioavailability and loss of perfused microvascular volume due to spillover of hyaluronidase may have affected peripheral resistance differently in the hyaluronidase treated goats compared to the control group.

Blood flow decreased significantly in time in both the control and hyaluronidase treated group. In the control group, the decrease in blood flow was related to a decrease in $\mathrm{MVO}_{2}$ and RPP, suggesting matching of coronary blood flow to metabolic needs. In contrast, in the hyaluronidase treated goats, $\mathrm{MVO}_{2}$ and $\mathrm{RPP}$ were not decreased, and the decrease in blood flow may have resulted from vasoconstriction due to an impaired shear dependent NO production in the coronary vasculature [150]. Decreases in coronary blood flow due to pharmacological blockade of NO synthesis were reported in swines [85], while no effect was observed in dogs after NO synthase blockade [53]. However, vasoconstriction after hyaluronidase seems evident when taken the difference in perfusion pressure, i.e. aorta pressure, between the two groups into account. Thus, coronary resistance (defined as $\mathrm{P}_{a o} / \mathrm{Q}_{c o r}$ ) increased from $1.7 \pm 0.2$ to $2.2 \pm 0.2 \mathrm{mmHg} / \mathrm{ml} / \mathrm{min} / 100 \mathrm{gr}$ after hyaluronidase, but remained constant after administration of the vehicle $(1.2 \pm 0.1$ and $1.3 \pm 0.1 \mathrm{mmHg} / \mathrm{ml} / \mathrm{min} / 100 \mathrm{gr}$ before and after vehicle administration, respectively). It was shown in swine that blockade of NO synthesis caused a reduction in coronary blood flow that was compensated by a $\sim 5 \%$ increase in extraction at rest and during exercise [85]. The change in coronary resistance seems to confirm the occurance of vasoconstriction after hyaluronidase. The increase in coronary resistance after hyaluronidase in the current study differs from the unaltered resistance in the study described in chapter 7 . In that study, the coronary circulation was perfused by means of a pressure controlled perfusion system and hyaluronidase infused by this system. In contrast, in the current study, intracoro- 
nary infusion of hyaluronidase or vehicle was performed by a catheter placed in the left main coronary artery. Since coronary blood flow was reduced both after infusion of enzyme and vehicle, we cannot ruled out that the presence of the catheter caused coronary blood flow to be reduced.

A noticeable difference between the study using swine [85] and our study using goats was the fractional oxygen extraction. Oxygen extraction in our study was over all heart rates $48 \pm 2$ and $52 \pm 2 \%(\mathrm{P}<0.05)$ at baseline and after hyaluronidase, respectively, while in the swine oxygen extraction was between 75 and $95 \%$ at rest and during heavy exercise. In our studies oxygen extraction was determined while the animals were under anesthesia. In dogs, however, Yusa and Obara found no effect of different intravenous anesthesia on oxygen extraction [167]. In contrast, a significant reduction in oxygen extraction has been obeserved in humans comparing oxygen extraction in patients while awake and during anesthesia [148]. Besides anesthesia, the vessel from which venous blood is being sampled could contribute to the lower oxygen extraction. The closer to the right atrium the coronary venous sampling occurs, the more progressive the outflow is diluted from tissues supplied by the circumflex artery. In our study we might have sampled closer to the right atrium compared to study by Merkus et al. [85] and the blood in our study could, as a result, be diluted with blood from the circumflex artery resulting in higher venous oxygen values.

\section{Oxygen exchange and glycocalyx}

Heart rate was controlled by pacing of the heart and increased from 80 BPM with steps of 30 BPM to 170 BPM. Samples were taken at different heart rates to determine whether the relation between metabolic oxygen demand of the heart and oxygen supply and exchange was altered after hyaluronidase. With increasing heart rate the increase in myocardial oxygen consumption in the control situation was matched by an increase in blood flow and arteriovenous oxygen extraction remained constant. After hyaluronidase infusion, the arteriovenous oxygen difference was increased across all heart rates, and increased with increasing heart rate.

Hyaluronidase increases endothelial glycocalyx accessibility for flowing plasma and macromolecules by degradation of hyaluronan, one of the main glycosaminoglycans of the glycocalyx $[47,142]$. In addition, blood-exclusion by the glycocalyx was shown to be impaired in cremaster muscle not only by enzymatic treatment of the glycocalyx 
with e.g., heparinase or hyaluronidase [29,47,142], but also during superfusion with the vasodilators adenosine, bradykinin and sodium nitroprusside [74, 110, 152, 154]. The role of glycocalyx modulation in oxygen transport from the lumen to the tissue has been unclear. The current data indicate that the glycocalyx may hinder oxygen exchange in the coronary circulation, and that a reduction in the glycocalyx barrier properties might favor oxygen exchange. In line with our observation Chen and coworkers [17] computed, using a diffusion-reaction model, that the presence of an endothelial glycocalyx in an arteriole could reduce the amount of oxygen diffusing into the tissue by about $8 \%$. Their model showed that the endothelial glycocalyx increased the resistance for diffusion toward the tissue from the center of the lumen. Since in their model the glycocalyx properties were assumed to be the same as those of plasma, the oxygen limitation was due to the increased distance between red blood cells and myocardial tissue. In the current study an increase in arteriovenous oxygen difference of approximately $14 \%$ was measured. Recruitment of the glycocalyx volume may be expected to decrease the distance between red blood cells and the myocardial tissue, and to increase the number of red blood cells within a capillary as reflected by an increase in capillary tube hematocrit. Both processes may facilitate the exchange of oxygen. It was already suggested that moment-to-moment variations in capillary tube hematocrit, in response to changes in oxygen content of the fluid superfusing the tissue and due to muscular contraction [28,74], change the oxygen supply to the tissue. An increase in capillary tube hematocrit might represent a higher instantaneous capillary oxygen transport capacity, and could increase the ability of the tissue to extract oxygen from red blood cells.

Another possible mechanism by which an intact endothelial glycocalyx might limit oxygen transport under control conditions is that oxygen is being scavenged within the glycocalyx. Xanthine oxidase, localized on the outside surface of endothelial cells [1], for instance, can scavenge oxygen and generate superoxide [1,121]. As a result, not all oxygen extracted from the blood will diffuse into the surrounding tissue. Partial removal of the glycocalyx may, however, increase the amount of oxygen scavenged by xanthine oxidase. Xanthine oxidase, previously shielded from contact with oxygen by the glycocalyx, might now be in contact with oxygen in the flowing blood and could scavenge more oxygen compared to baseline, increasing the arteriovenous oxygen difference. 
Since RPP and $\mathrm{MVO}_{2}$ were not altered after hyaluronidase, the increase in oxygen extraction and reduction in blood flow seem to nicely match each other. The measurement can, however, not distinguish between the increase in oxygen extraction being the cause or result of the decrease in blood flow. More experiments are required to determine to what extent glycocalyx recruitment contributes to the increase in oxygen exchange. Measuring oxygen exchange at different levels of exercise in the presence and absence of the glycocalyx would provide us with data of oxygen exchange at higher work loads of the heart. If vasoconstriction after hyaluronidase, caused by impaired shear induced vasodilation, caused the higher oxygen extraction, the oxygen exchange will most likely be insufficient at higher working loads.

\section{Conclusion}

In conclusion, we demonstrate in the current study in anesthetized goats that after hyaluronidase treatment of the endothelial glycocalyx in the coronary vasculature there is an increase in oxygen exchange of the heart. Our data indicate that the loss of the endothelial glycocalyx might contribute to the increase in oxygen exchange, and that recruitment of the glycocalyx could be an additional mechanism by which the heart can match oxygen demand and extraction. Although from this study glycocalyx loss might seem beneficial for microvascular perfusion, degradation of the glycocalyx has been associated with an increase in platelet adhesion [115], edema formation [142], impaired flow-mediated dilation [90, 104], and loss of capillary perfusion [12, 172], which ultimately will cause microvascular perfusion to be compromised. On the other hand, if the glycocalyx truly constitutes a barrier for oxygen exchange and this barrier could be reduced transiently when needed, e.g. by acute agnoinst-induced recruitment of glycocalyx volume, glycocalyx modulation might contribute to physiological control of oxygen exchange.

\section{Acknowledgments}

The authors would like to thank Marion Kuiper, Anniek Lampert, and Arne van Hunnik for their assistance. 


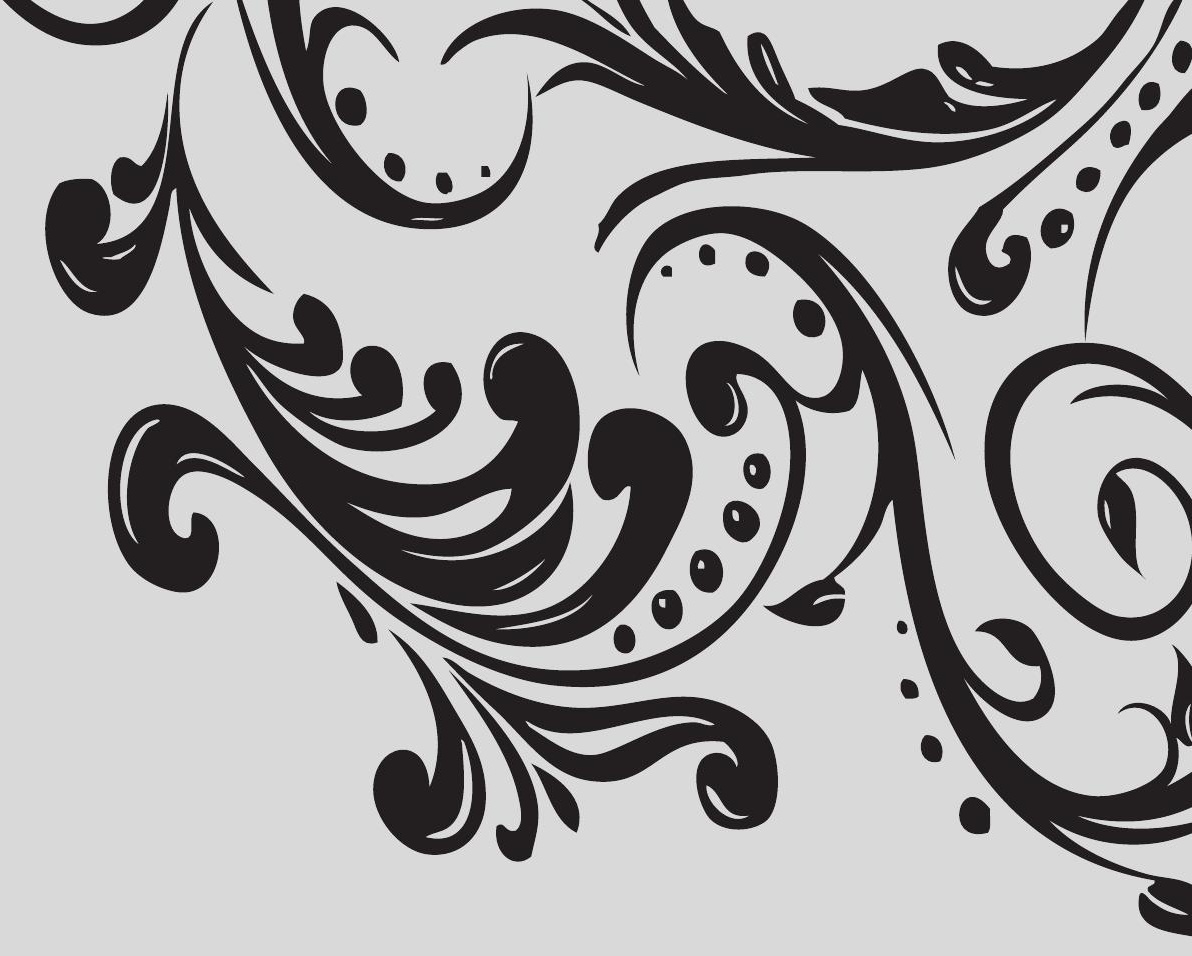

Discussion 



\section{Discussion}

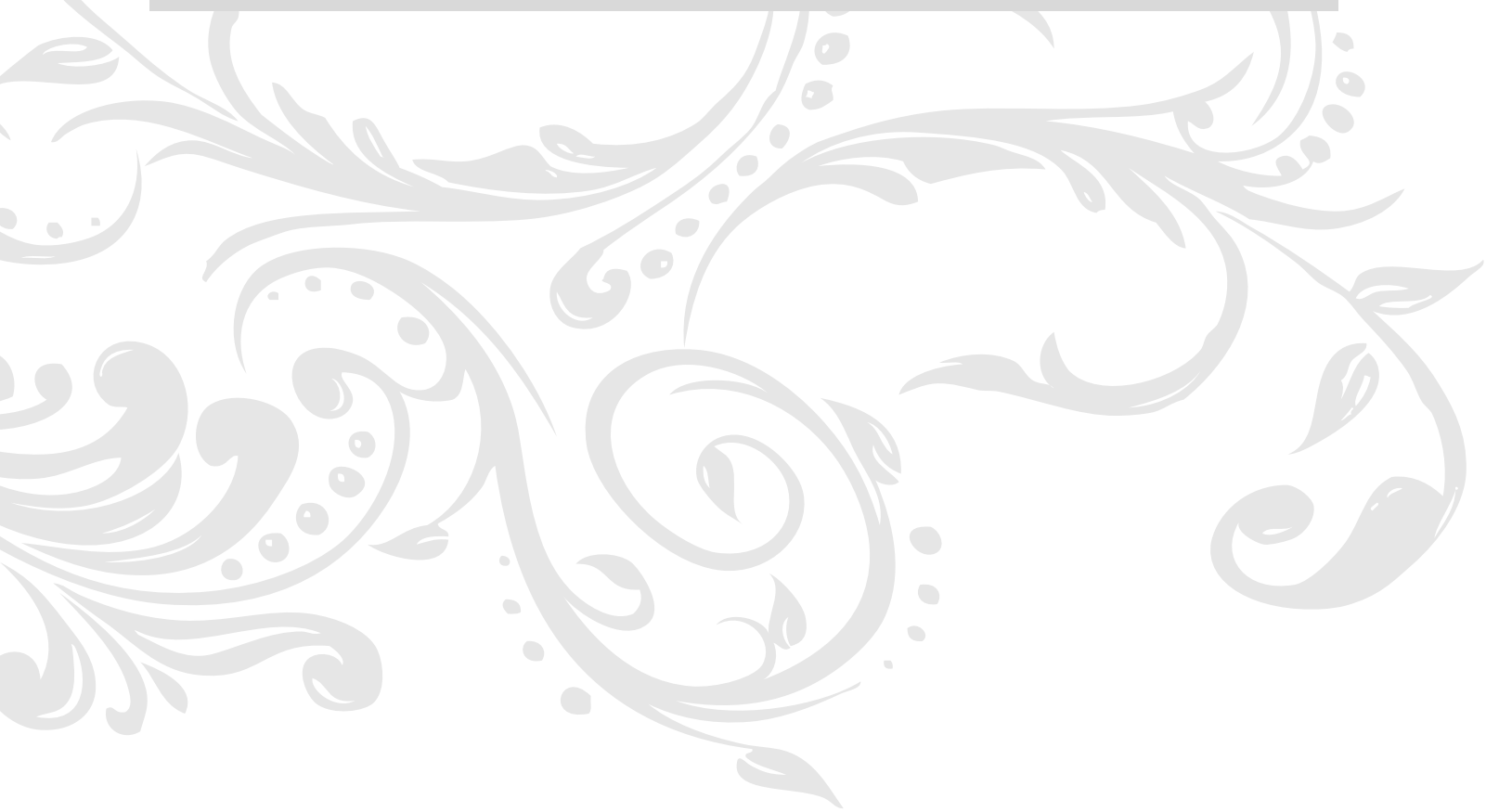




\title{
Agonist-induced modulation of glycocalyx barrier properties in the microcirculation
}

\author{
Role in blood volume recruitment and oxygen exchange in \\ the heart
}

There is a considerable number of patients with symptoms of chest pain during exercise who do not have a prominent stenosis in their coronary arteries or coronary artery disease at all [62]. Furthermore, a large number of the patients with a detectable lesion have a hemodynamically non-significant intermediate coronary artery stenosis [109]. In all these cases, the angina may relate to coronary microvascular dysfunction [13]. In the microvasculature a substantional part of the anatomic vascular volume is occupied by blood-excluding glycocalyx [47,100,103,110,111,121,152,157,158]. Degradation of the glycocalyx in the microvasculature is associated with an impaired shear-dependent NO-production, an increased endothelial adhesion of leukocytes and platelets, an increased leakage of water and proteins from the vasculature, and a reduction in number of perfused capillaries $[10,151]$. These microvascular problems may ultimately hinder an adequate supply and exchange of oxygen to the cardiomyocytes, and underlie the angina [13].

The diagnosis of coronary artery disease is done mainly on flow reserve, determined using adenosine, and angiography. Duling and coworkers [29,74,110] have suggested that adenosine, in addition to relaxation of the resistance vessels, affects the exclusion properties of the glycocalyx. In this way adenosine may increase functionally perfused capillary volume by "recruitment" of glycocalyx volume. The contribution of glycocalyx modulation to coronary blood volume during adenosine was, however, never studied. The aim of our project was, therefore, to investigate (1) the effect of adenosine on glycocalyx exclusion of circulating blood in the systemic and coronary circulation and (2) whether glycocalyx recruitment might present a mechanism by which vasoactive substances can influence blood flow / blood volume, and whether this affects oxygen exchange in the heart.

During the infusion of adenosine no measurable blood-excluding glycocalyx volume seemed to be left (systemic and coronary). In the systemic circulation the recruitment of glycocalyx was associated with an equal decrease in anatomic vascular volume, in line with the hypothesis that adenosine impairs blood exclusion by the glycocalyx. 
Intracoronary adenosine infusion resulted in an increase in coronary blood perfused volume confirming the hypothesis that adenosine can increase functionally perfused volume by recruiting glycocalyx volume, see figure 9.1. The contribution of recruitment of glycocalyx to the increase in blood volume, besides vasodilation of the resistance arterioles, has consequences for the general view of how adenosine may increase perfusion of the heart. Furthermore, in the case of glycocalyx degradation changes in coronary blood flow were no longer accompanied by a change in coronary blood volume. The uncoupling of flow from volume reserve in case of glycocalyx degradation in combination with impaired vascular homeostasis in both macro- and microcirculation might be associated with an inefficient uptake of supplied oxygen. These data suggest the need for monitoring volume reserve capacity in addition to assessment of flow reserve in patients with coronary artery disease.

\section{Agonist-induced glycocalyx modulation}

Adenosine has been suggested in the literature to increase glycocalyx accessibility for plasma macromolecules. Our first aim was to study whether vasodilators other than adenosine would have an effect on glycocalyx exclusion properties as well (chapter 3). Using intravital microscopy the effects of bradykinin and sodium nitroprusside were determined in mouse cremaster muscle on capillary tube hematocrit and glycocalyx barrier properties. During infusion of both vasodilators in the presence of an intact glycocalyx there was a reduction in glycocalyx exclusion of $70 \mathrm{kDa}$ dextrans and an increase in capillary tube hematocrit. These data illustrate that functionally perfused capillary volume can be increased by agonist-dependent modulation of glycocalyx exclusion. However, these studies do not provide insight about what happens at the whole body or individual organ level. Our second aim was, therefore, to study the effect of adenosine on glycocalyx exclusion at the whole body level using the methodology used for measurement of systemic blood and glycocalyx volume in humans $[100,102,104]$. This method was first validated in mice by evaluating the effect of provoked glycocalyx loss on the measurement (chapter 4), and it was shown that the whole-body blood-excluding glycocalyx volume measurement using dilution of labeled red blood cells and large vessel hematocrit is susceptible to enzymatic glycocalyx provocation. Subsequently, anatomic and blood perfused vascular volume were measured in goats at baseline and during an intravenous infusion of adenosine 


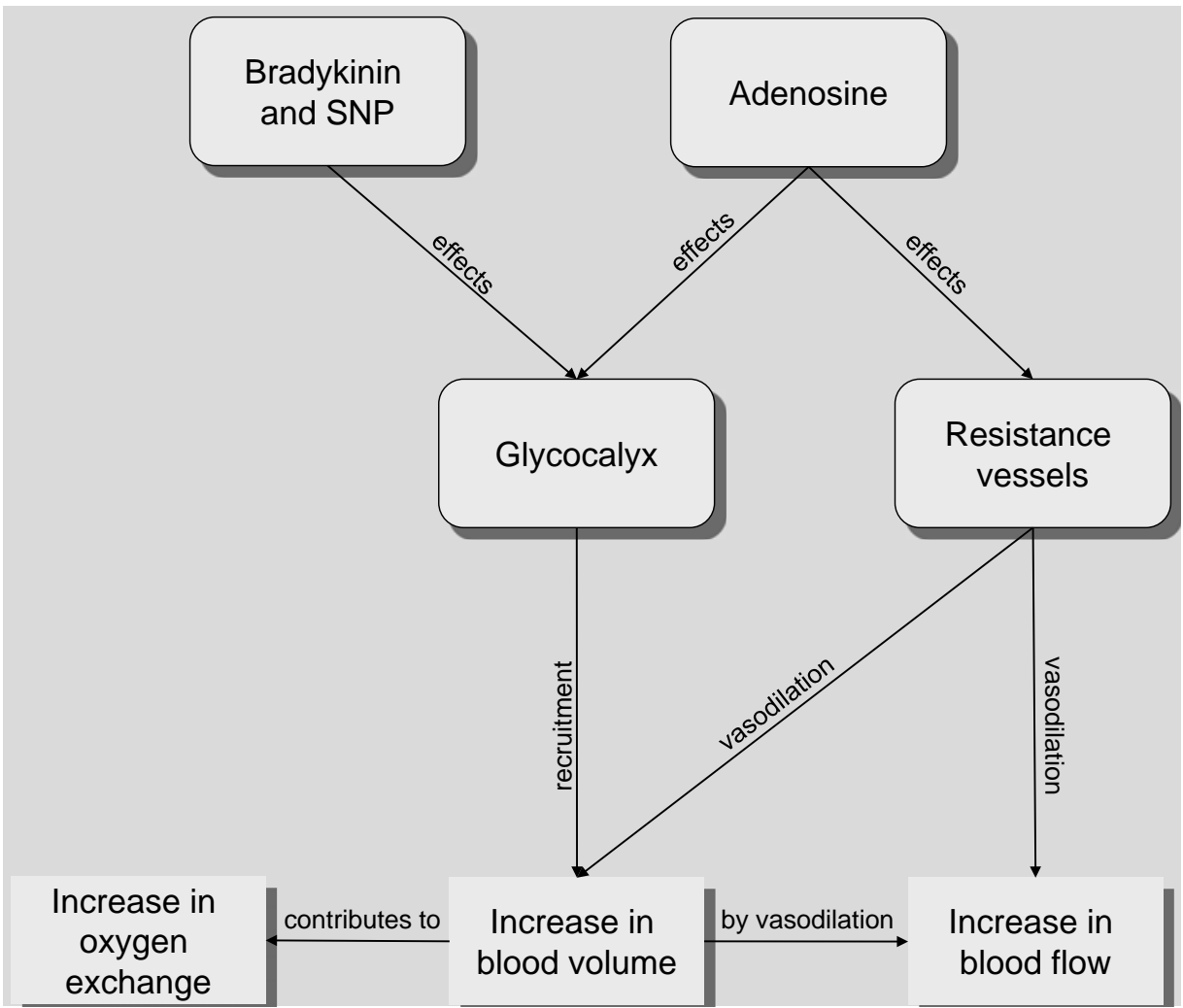

Figure 9.1 Vasodilator induced modulation of the glycocalyx results in reduced glycocalyx exclusion in capillaries (increased functionally perfused volume), and an increased coronary blood volume and oxygen exchange in the heart.

(chapter 5). At baseline there was a glycocalyx volume of $\sim 600 \mathrm{ml}$. During intravenous administration of adenosine the glycocalyx volume was almost completely vanished, illustrating that adenosine decreases blood exclusion by the glycocalyx. The reduction in systemic glycocalyx volume was not associated with an increase in circulating blood volume but with a reduction in tracer accessible volume, suggesting redistribution of blood and a loss in the number of perfused capillaries [12,172]. Our next step was to study the effect of adenosine on the level of the heart. Coronary blood volume was measured with the indicator-dilution-technique using labeled red blood cells and large dextrans as plasma tracer. Since there are many different models 
available that can be used to analyze the indicator dilution data, four models were compared to determine which model can best be used to analyze coronary indicator dilution curves. The models analyzed coronary indicator dilution curves at baseline and during adenosine administration (chapter 6). Each model fitted a curve through the data and using this curve the average time it takes for the tracer injected to travel from point of injection to point of sampling (MTT) could be determined. Multiplying the MTT with the tracer flow gives the distribution volume of the indicator. There was little difference between the models with the exception of the power-law model, providing considerable larger estimations of coronary blood volumes as the other three models and data reported in literature. Since the LDRW model was one of the models that was least sensitive to noise and recirculating tracer this model was chosen to analyze coronary indicator dilution curves. Coronary blood volume was measured at baseline and during adenosine administration before and after hyaluronidase treatment (chapter 7). With an intact glycocalyx adenosine infusion resulted in a robust increase in both coronary blood flow and volume. After hyaluronidase treatment of the glycocalyx baseline coronary blood volume increased without a change in blood flow while subsequent adenosine administration was associated with a similar increase in coronary blood flow as before treatment but an attenuated increase in coronary blood volume. Further, a trend was observed that myocardial oxygen consumption was reduced during adenosine infusion after hyaluronidase treatment, suggesting that the glycocalyx could play a role in the control of oxygen exchange in the heart. To test how glycocalyx degradation would affect coronary oxygen exchange, myocardial oxygen extraction was determined at baseline and after hyaluronidase treatment of goat hearts that were paced at different heart rates (chapter 8). The difference in oxygen content in arterial and coronary venous blood increased after hyaluronidase treatment, indicating an increase in oxygen extraction. These data indicate that glycocalyx volume recruitment may facilitate the exchange of oxygen in the heart.

\section{Implications of a damaged glycocalyx for the regulation of blood flow, volume, and oxygen exchange in the heart}

The observation that enzymatic degradation of the glycocalyx was associated with distinct changes in coronary blood volume and flow during baseline versus adenosine 


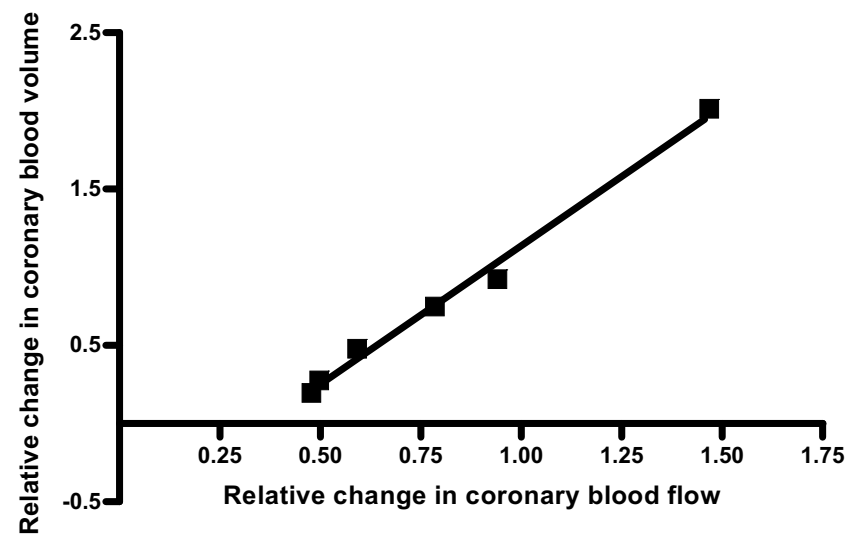

(a) Baseline

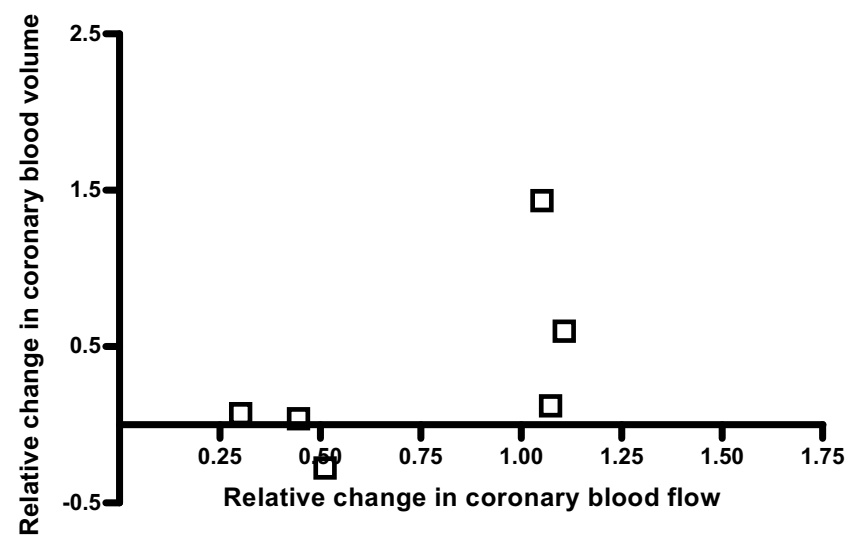

(b) Hyaluronidase

Figure 9.2 Change in coronary blood volume and flow normalized to corresponding baseline measurements. With an intact glycocalyx (a) an increase in flow is associated with an increase in coronary blood volume $\left(\mathrm{R}^{2}=0.99\right)$. After hyaluronidase $(\mathrm{b})$ the relation between a change in flow and volume is impaired $\left(\mathrm{R}^{2}=0.40\right)$.

infusion suggest that both parameters can vary independently of each other, see figure 9.2. Coronary blood volume increases appear to depend on the recruitment of glycocalyx volume and dilation of the resistance vessels while coronary flow is primarily increased by relaxation of resistance arterioles only. Increases in coronary blood flow 
and volume seem to occur in a coordinated manner, however, when the glycocalyx is intact. The glycocalyx might play, therefore, an essential role in matching concomitant changes in flow and volume; this coupling is illustrated by the maintenance of MTT during adenosine administration in our experiments. In contrast, glycocalyx degradation was associated with a mismatch in the increase in flow versus the increase in volume and a reduction in MTT during adenosine compared to corresponding baseline. We hypothesize, therefore, that recruitment of glycocalyx volume enables agonists to couple nutrient exchange in the capillaries (by augmenting surface area and controlling MTT) to increases in nutrient delivery by the blood flow (via vasodilation of resistance vessels), e.g. during exercise. While the results in chapter 8 indeed showed that recruitment of glycocalyx volume increase myocardial oxygen extraction, the data also indicate that in anesthetized goats, increases in myocardial oxygen consumption at low workloads (i.e., pacing of the heart) are primarily matched by an increase in blood flow. Further studies are needed to determine whether at higher workloads of the heart, such as are obtained during whole-body exercise, recruitment of glycocalyx volume also contributes to the adequate regulation of myocardial oxygen exchange.

\section{Methodological considerations}

Hemodynamics Experiments described in this thesis were performed on goats; in chapter 7 (coronary glycocalyx measurement) relative small goats were used $(\sim 20 \mathrm{~kg})$ and in chapter 8 (oxygen exchange) large goats $(50-60 \mathrm{~kg}$ ) were used. When comparing the data from chapter 7 with the data from chapter 8 a noteworthy difference can be found. The flow (per 100 gram of heart tissue) measured in chapter 7 was 3 to 4 times higher than the flow in chapter 8 . The higher coronary blood flow can be explained mainly by a higher myocardial oxygen consumption $\left(\mathrm{MVO}_{2}\right)$ in chapter 7 (figure 9.3), see below. Furthermore, the arterial oxygen content was significantly lower in chapter 7 (range $5.8-14.3 \mathrm{ml} / 100 \mathrm{ml}$ ) compared to chapter 8 (range 11.3-21.7 $\mathrm{ml} / 100 \mathrm{ml}$ ), and this may have resulted in a lower $\mathrm{AV}_{o x}$ (range 3.1-6.6 ml/100ml) and, concomitantly a higher coronary blood flow. The fractional oxygen extraction was, however, not significantly different, when comparing both studies. Fractional oxygen extraction at baseline was $57.1 \pm 5.0 \%$ and $48.2 \pm 2.1 \%$ in chapter 7 and 8 , respectively. After hyaluronidase administration oxygen extraction was $51.8 \pm 3.4 \%$ in chapter 7 and $51.9 \pm 2.2 \%$ in chapter 8 . 


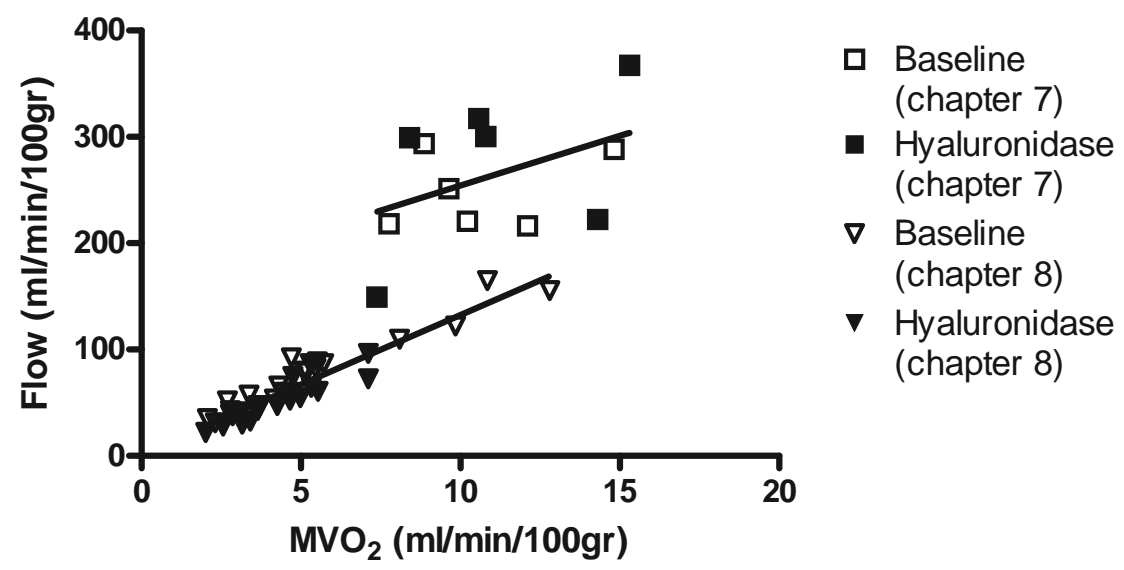

Figure 9.3 Myocardial oxygen consumption $\left(\mathrm{MVO}_{2}\right)$ versus coronary blood flow at baseline (closed symbols) and after hyaluronidase (open symbols) for the data of chapter 7 (squares) and 8 (triangles).

The work load of the hearts (defined as the rate-pressure-product, RPP) in chapter 7 were approximately $50 \%$ higher compared to the RPP values of chapter 8 resulting in a higher $\mathrm{MVO}_{2}$. Additionally, the relation between myocardial oxygen consumption and RPP was different in chapter 7 compared to chapter 8, see figure 9.4. The higher RPP values might have been caused by the use of a perfusion system and not pacing of the hearts, resulting in higher heart rates. The altered relation between RPP and $\mathrm{MVO}_{2}$ might be explained by pacing of the hearts in chapter 8. A study by Kal et al. [60] demonstrated that pacing induced changes in $\mathrm{MVO}_{2}$ cannot be predicted accurately from changes in RPP. In their study they found that the regression of $\Delta \% \mathrm{MVO}_{2}$ to $\triangle \% \mathrm{RPP}$ was statistically significant. However, it was indicated that the clinical relevance of this relation is quite a different matter, because only $40-60 \%$ of the variation in $\Delta \% \mathrm{MVO}_{2}$ could be explained by the variation in $\Delta \% \mathrm{RPP}$.

Coronary blood flow was unaltered after vehicle or enzyme administration in chapter 7 while a reduction in coronary blood flow was observed in chapter 8 . The difference in effect on coronary blood flow in the different studies could be caused by the difference in instrumentation (presence/absence of perfusion system, different anesthesia, and open versus closed chest) as well as the method of administration of the vehicle/enzyme. In chapter 7 we used a pressure controlled perfusion system which 


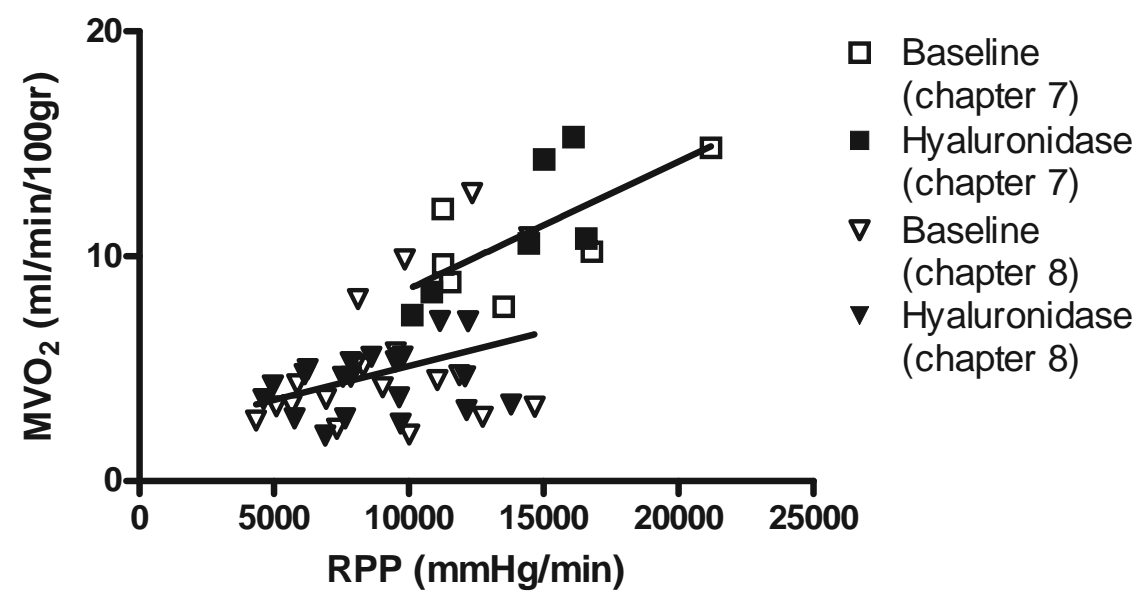

Figure 9.4 Rate-pressure-product (RPP) versus myocardial oxygen consumption $\left(\mathrm{MVO}_{2}\right)$ at baseline (closed symbols) and after hyaluronidase (open symbols) for the data of chapter 7 (squares) and 8 (triangles).

enabled easy intracoronary administration via the perfusion line. In contrast, in chapter 8 , a catheter $(7 \mathrm{~F})$ was placed in the left main for 50 minutes for administration of vehicle/enzyme, and this might have interfered with the coronary blood flow.

When comparing the hemodynamic parameters of chapter 7 and 8 with each other another difference can be observed. Although a reduction in blood pressure in the vehicle group cannot be statistically tested $(\mathrm{N}=3$, chapter 7$)$ there was a trend that the blood pressure after vehicle administration was lower then the pressure measured at baseline. In contrast, no reduction in blood pressure was observed in the hyaluronidase group. In chapter 8 a similar observation can be made. Blood pressure across all heart rates after vehicle infusion was significantly reduced compared to baseline while the blood pressure before and after hyaluronidase treatment did not differ. Loss of number of functionally perfused capillaries or impaired shear induced NO production could have resulted in the higher blood pressure after hyaluronidase compared to vehicle administration. Although the hyaluronidase is diluted in the circulating blood and has a short half life (<10min [165]), the enzyme has been given for 50 minutes and effects on the systemic circulation can not be ruled out. 
Microvascular blood volume During intracoronary adenosine infusion coronary blood volume increased, however, when adenosine was given systemically, total blood volume remained unaltered and anatomic blood volume was reduced. We suggest that in organs, such as the heart, in which blood flow is increased, the microvascular blood volume will increase as well (recruitment of microvascular volume). In other organs there is a reduction in blood flow (such as adipose tissue, kidney, liver and stomach $[31,66])$ and we expect that this is associated with loss of blood perfused volume. To test the hypothesis that the effect of intravenous adenosine administration is different in different organs the number of total and perfused capillaries needs to be counted in different organs at baseline and during intravenous adenosine administration. The number of perfused capillaries can be determined using labeled tracers such as labeled red blood cells and dextrans with a molecular weight of $2000 \mathrm{kDa}$ (Dex-2000), while the total number of capillaries present can be counted after staining of the sections using an endothelial marker.

Tail of the indicator dilution curve In literature various different models have been used to describe indicator dilution curves and four of these models were compared with each other in chapter 6 after which one model was chosen to analyze the indicator dilution curves of chapter 7 . The selection of the models that were used in chapter 6 was based on the frequency that the model was used in literature, the monoexponential and gamma-variate model $[7,41,42,49,81,91,168]$, or on very promising results published by others, the power-law and local density random walk model $[4,88]$. The choice which model was best suited to analyze the coronary indicator dilution curve data was based on feasibility and signal to noise ratio. Due to the complexity of the coronary circulation no in vitro setup was available to test how accurate the estimated volumes of the different models were. The accuracy of the models was only based on in literature reported coronary blood volumes. Therefore, the estimated volumes of the models played a limited role in the choice of which model would be used. Interestingly, all four models tested provided a fit through the data in which the tail of the curve was below the measured data. This cannot be explained by recirculation of the tracer used to measure coronary blood volume since due to the use of a perfusion system recirculation was delayed by more than 10 seconds. When more data of the descending slope was selected as input for the local density random walk model, 
as was done in chapter 7 , the difference between the model and the measured data became less, nevertheless a difference between the data and model at the lower part of the curve remained. A possible cause for the difference between the model and measured data is the organ of interest, the coronary vasculature. Bassingthwaighte and coworkers have studied indicator dilution curves in single vessels, and in the coronary, renal and pulmonary circulation. They suggested that the late, slowly decreasing tails observed when studying the coronary circulation, causing a difference between model and measured data, could only be explained by the coronary bed itself [75]. For example, local arterial reversed flow with pulsatile intramyocardial tension development could cause changes in the tail of transfer curves that can not be described accurately by any of the models. Mischi et al. [88] found in an in vitro setup and in patients (left and right ventricle) using the local density random walk fit a good agreement between the measured and estimated data, including the lower part of the curves, supporting the idea that the coronary bed, in stead of the choice of model, causes the difference between model and measured data.

Use of small dextrans to probe the glycocalyx In the systemic circulation (chapter 5) dextrans with a MW of $40 \mathrm{kDa}$ (Dex-40) were used to determine the anatomic vascular volume. The difference between Dex-40 and circulating plasma volume (derived from red blood cell volume and large vessel hematocrit) was defined as systemic glycocalyx volume. In the coronary circulation (chapter 7 ) glycocalyx volume was defined as the difference in coronary blood volume at baseline and after hyaluronidase treatment. Unlike in the systemic circulation, Dex-40 appeared not useful for delineating glycocalyx volume in the coronary circulation. When comparing the mean transit time of Dex-40 with that of Dex-2000, our plasma tracer, no difference was found, see figure 9.5 for a typical example. A possible cause could be that Dex-40 has no instantaneous access to the glycocalyx in the coronary circulation as was suggested by the intravital microscopy studies in the cremaster [158]. In these studies Dex-40 was found to fill the entire intravascular volume at the first measurement point, which was 60 seconds after injection; however there were no measurements of Dex-40 within the first few seconds after tracer application. Another option is that Dex-40 does have instantaneous access to the glycocalyx but that back-diffusion into the circulating blood is so rapid that it does not significantly affect the mean transit 




Figure 9.5 A typical example of normalized red blood cell (RBC) concentration curve (gray diamonds), FITC labeled dextrans with a MW of $2000 \mathrm{kDa}$ (black circles) and TR labeled dextrans with a MW of $40 \mathrm{kDa}$ (black crosses). There is no difference in concentration outflow curves of dextrans with a MW of $40 \mathrm{kDa}$ and $2000 \mathrm{kDa}$.

time.

During adenosine administration we observed a significant increase in Dex-40 concentration, parallel to the decrease in glycocalyx volume, illustrating a reduction in distribution volume of Dex-40 (chapter 5). A comparable smaller Dex-40 distribution volume has also been observed during glycocalyx loss in persons during hyperglycemia and in patients with diabetics $[103,104]$. Our first blood sample was taken after 3 minutes and the initial Dex-40 concentration was determined by back-extrapolation of the measured samples. The estimated Dex-40 could be an underestimation of the actual concentration at the moment of injection. Dex-40 consist of a mixture of dextrans of different molecular weights, and within the first 3 minutes after injection of the tracer the smaller dextrans could have been cleared from the vasculature, result- 
ing in a lower initial Dex-40 concentration. This was also suggested in a commentary by Michel and Curry [86]. Although a reduced clearance of the small dextrans due to renal vasoconstriction could have contributed to an increase in Dex-40 concentration during adenosine, this would likely have resulted in a different exponential coefficient, something we did not observe. Instead, we suggested that the smaller distribution volume of Dex-40 truly represents a reductioon in anatomic microvascular volume due to loss of glycocalyx.

Finally, in literature, plasma distribution volume derived from the dilution of red blood cells and large vessel hematocrit has been suggested to result in an underestimation of the true circulating plasma volume because of a lower hematocrit in the microcirculation compared to the large vessels [86]. Thus, volumes estimated using red blood cells and large vessel hematocrit have resulted in values smaller than the volumes estimated using albumin as circulating plasma tracer. As a consequence the glycocalyx volume determined using albumin was found to be smaller than those based on red blood cell volume measurements [103,104]. It has been shown, however, that albumin is not fully excluded from the glycocalyx [55,158], and as a consequence, volume measurements using albumin likely result in an overestimation of the circulating plasma volume. Instead, use of large molecular weight tracer, such as Dex-2000, which is anticipated to be fully excluded from the glycocalyx, is preferred. In this respect, estimations of the plasma volume in humans using labeled hydroxyethyl starch corresponded nicely to the estimation of the plasma volume derived from dilution of labeled red blood cells and hematocrit [82].

\section{Future perspectives}

Our studies imply that the view of how adenosine regulates coronary blood flow and coronary blood volume needs to be reconsidered. As a result, it might be appropriate to measure volume reserve in addition to flow reserve. Before measurements in patients can be done the measurement of coronary volume reserve needs to be translated from bench to bedside. A possible method is by imaging the blood volume using myocardial contrast echo (MCE). The contrast agents used in MCE, microbubbles, have an intravascular rheology similar to that of red blood cells [69]. Microbubbles can be used as a magnetic resonance (MR) susceptibility contrast agent as well [18]. Gas-filled microbubbles induces a large local magnetic proneness difference by the 
gas-liquid interface [18]. MRI or echo-derived microvascular volume reserve might be used as diagnostic tool to image non-invasively glycocalyx loss in the coronary circulation (by comparing coronary blood volume during adenosine to coronary blood volume at baseline, as was done in chapter 7), which may well be present before the detection of significant coronary vascular disease. In the near future a collaboration with University of Pittsburgh Medical Center will start to determine whether changes in coronary blood volume can be detected accurately using a non-invasive technique, and whether a reduced coronary volume reserve is associated with an increased risk for coronary artery disease. To do so, coronary blood volume using MCE will be measured in control subjects, in patients with coronary artery disease (CAD) with and without angina, in patients who have had a myocardial infarction, and in patients with type I diabetes with and without microalbuminuria.

Last, patients could benefit from treatment aimed at glycocalyx preservation. It has been suggested that glycocalyx loss may be secondary to a direct effect of oxygen radicals on the synthesis of glycosaminoglycans [104]. Blocking of radical production by free radical scavengers or pharmacological blockers of radical production might, therefore, be useful to diminish the oxygen radical stress on the glycocalyx [149]. Prevention of glycocalyx loss by administration of drugs that might preserve the glycocalyx [16] or by preventing proteolysis of the glycocalyx [101] might also be promising therapeutic targets. Finally, it was already shown in animals that the administration of glycocalyx components, such as heparan sulfates, heparin and hyaluronan, could also reduce the adverse effects seen during loss of glycocalyx [21,121]. 


\section{References}

[1] Adachi, T., Fukushima, T., Usami, Y., and Hirano, K. Binding of human xanthine oxidase to sulphated glycosaminoglycans on the endothelial-cell surface. Biochem J 289 ( Pt 2) (1993), 523-7.

[2] Adamson, R. H., And Clough, G. Plasma proteins modify the endothelial cell glycocalyx of frog mesenteric microvessels. J Physiol 445 (1992), 473-86.

[3] Allison, D. D., And Grande-Allen, K. J. Review. Hyaluronan: a powerful tissue engineering tool. Tissue Eng 12, 8 (2006), 2131-40.

[4] Bassingthwaighte, J. B., And Beard, D. A. Fractal ${ }^{15}$ O-labeled water washout from the heart. Circ Res rry, 6 (1995), 1212-21.

[5] Bassingthwaighte, J. B., Knopp, T. J., And Anderson, D. U. Flow estimation by indicator dilution (bolus injection). Circ Res 27, 2 (1970), 27791.

[6] Bishop, J. R., Schuksz, M., And Esko, J. D. Heparan sulphate proteoglycans fine-tune mammalian physiology. Nature 446, 7139 (2007), 1030-7.

[7] Boganrd, J. M., Smith, S. J., Versprille, A., Wise, M. E., And HageMEIJER, F. Physiological interpretation of the skewness of indicator-dilution 
curves; theoretical considerations and a practical application. Basic Res Cardiol 79, 4 (1984), 479-93.

[8] Bogahrd, J. M., van Duyl, W. A., Versprille, A., and Wise, M. E. Influence of random noise on the accuracy of the indicator-dilution method. Clin Phys Physiol Meas 6, 1 (1985), 59-64.

[9] Bosman, J., Tangelder, G. J., Oude Egbrink, M. G., Reneman, R. S., AND SlaAf, D. W. Capillary diameter changes during low perfusion pressure and reactive hyperemia in rabbit skeletal muscle. Am J Physiol 269, 3 Pt 2 (1995), H1048-55.

[10] Brands, J., Van Teeffelen, J. W., Van den Berg, B. M., And Vink, H. Role for glycocalyx perturbation in atherosclerosis development and associated microvascular dysfunction. Future Lipidology 2, 5 (2007), 527-534.

[11] Bruegger, D., Jacob, M., Rehm, M., Loetsch, M., Welsch, U., Conzen, P., And Becker, B. F. Atrial natriuretic peptide induces shedding of endothelial glycocalyx in coronary vascular bed of guinea pig hearts. Am J Physiol Heart Circ Physiol 289, 5 (2005), H1993-9.

[12] Cabrales, P., Vazquez, B. Y., Tsai, A. G., and Intaglietta, M. Microvascular and capillary perfusion following glycocalyx degradation. $J$ Appl Physiol 102, 6 (2007), 2251-9.

[13] Camici, P. G., And Crea, F. Coronary microvascular dysfunction. $N$ Engl J Med 356, 8 (2007), 830-40.

[14] Canty, J. M., J., Judd, R. M., Brody, A. S., And Klocke, F. J. Firstpass entry of nonionic contrast agent into the myocardial extravascular space. Effects on radiographic estimates of transit time and blood volume. Circulation 84, 5 (1991), 2071-8.

[15] Casella, G., Leibig, M., Schiele, T. M., Schrepf, R., Seelig, V., Stempfle, H. U., Erdin, P., Rieber, J., Konig, A., Siebert, U., And KLAUss, V. Are high doses of intracoronary adenosine an alternative to standard intravenous adenosine for the assessment of fractional flow reserve? $\mathrm{Am}$ Heart J 148, 4 (2004), 590-5. 
[16] Chappell, D., Jacob, M., Hofmann-Kiefer, K., Bruegger, D., Rehm, M., Conzen, P., Welsch, U., And Becker, B. F. Hydrocortisone preserves the vascular barrier by protecting the endothelial glycocalyx. Anesthesiology 107,5 (2007), 776-84.

[17] Chen, X., Jaron, D., Barbee, K. A., and Buerk, D. G. The influence of radial $\mathrm{RBC}$ distribution, blood velocity profiles, and glycocalyx on coupled NO/O 2 transport. J Appl Physiol 100, 2 (2006), 482-92.

[18] Cheung, J. S., Chow, A. M., Guo, H., And Wu, E. X. Microbubbles as a novel contrast agent for brain MRI. Neuroimage 46, 3 (2009), 658-64.

[19] Clark, M. G. Impaired microvascular perfusion: a consequence of vascular dysfunction and a potential cause of insulin resistance in muscle. Am J Physiol Endocrinol Metab 295, 4 (2008), E732-50.

[20] Constantinescu, A. A., Vink, H., And Spann, J. A. Elevated capillary tube hematocrit reflects degradation of endothelial cell glycocalyx by oxidized LDL. Am J Physiol Heart Circ Physiol 280, 3 (2001), H1051-7.

[21] Constantinescu, A. A., Vink, H., And Spann, J. A. Endothelial cell glycocalyx modulates immobilization of leukocytes at the endothelial surface. Arterioscler Thromb Vasc Biol 23, 9 (2003), 1541-7.

[22] Crystal, G. J., Downey, H. F., And Bashour, F. A. Small vessel and total coronary blood volume during intracoronary adenosine. Am J Physiol 241, 2 (1981), H194-201.

[23] Crystal, G. J., Rooney, M. W., and Salem, M. R. Myocardial blood flow and oxygen consumption during isovolemic hemodilution alone and in combination with adenosine-induced controlled hypotension. Anesth Analg 67, 6 (1988), $539-47$.

[24] Damiano, E. R. The effect of the endothelial-cell glycocalyx on the motion of red blood cells through capillaries. Microvasc Res 55, 1 (1998), 77-91. 
[25] Damon, D. H., And Duling, B. R. Distribution of capillary blood flow in the microcirculation of the hamster: an in vivo study using epifluorescent microscopy. Microvasc Res 27, 1 (1984), 81-95.

[26] Damon, D. H., And Duling, B. R. Evidence that capillary perfusion heterogeneity is not controlled in striated muscle. Am J Physiol 249, 2 Pt 2 (1985), H386-92.

[27] Damon, D. H., And Duling, B. R. Are physiological changes in capillary tube hematocrit related to alterations in capillary perfusion heterogeneity? Int J Microcirc Clin Exp 6, 4 (1987), 309-19.

[28] Desjardins, C., And Duling, B. R. Microvessel hematocrit: measurement and implications for capillary oxygen transport. Am J Physiol 252, $3 \mathrm{Pt} 2$ (1987), H494-503.

[29] Desjardins, C., And Duling, B. R. Heparinase treatment suggests a role for the endothelial cell glycocalyx in regulation of capillary hematocrit. Am J Physiol 258, 3 Pt 2 (1990), H647-54.

[30] Duncker, D. J., And Merkus, D. Acute adaptations of the coronary circulation to exercise. Cell Biochem Biophys 43, 1 (2005), 17-35.

[31] Edlund, A., Sollevi, A., And Linde, B. Haemodynamic and metabolic effects of infused adenosine in man. Clin Sci (Lond) 79, 2 (1990), 131-8.

[32] Feigl, E. O. Coronary physiology. Physiol Rev 63, 1 (1983), 1-205.

[33] Ferreira, L. F., Padilla, D. J., Musch, T. I., And Poole, D. C. Temporal profile of rat skeletal muscle capillary haemodynamics during recovery from contractions. J Physiol 573, Pt 3 (2006), 787-97.

[34] Florian, J. A., Kosky, J. R., Ainslie, K., Pang, Z., Dull, R. O., And TARBELl, J. M. Heparan sulfate proteoglycan is a mechanosensor on endothelial cells. Circ Res 93, 10 (2003), e136-42.

[35] Frank, J. S., And Langer, G. A. The myocardial interstitium: its structure and its role in ionic exchange. J Cell Biol 60, 3 (1974), 586-601. 
[36] Goldsmith, H. L., Cokelet, G. R., and Gaentgens, P. Robin Fahraeus: evolution of his concepts in cardiovascular physiology. Am J Physiol 25\%, $3 \mathrm{Pt}$ 2 (1989), H1005-15.

[37] Gonzalez-Castillo, C., Rubio, R., and Zenteno-Savin, T. Coronary flow-induced inotropism is modulated by binding of dextrans to the endothelial luminal surface. Am J Physiol Heart Circ Physiol 284, 4 (2003), H1348-57.

[38] Gouverneur, M., Spann, J. A., Pannekoek, H., Fontijn, R. D., And VINK, H. Fluid shear stress stimulates incorporation of hyaluronan into endothelial cell glycocalyx. Am J Physiol Heart Circ Physiol 290, 1 (2006), H458-2.

[39] Gunji, H., Little, R. A., And Hiraiwa, K. Interleukin-6 deficiency increases blood volume without altering body composition in young mice. Cytokine 20, 1 (2002), 30-7.

[40] Habazettl, H., Vollmar, B., Christ, M., Baier, H., Conzen, P. F., And Peter, K. Heterogeneous microvascular coronary vasodilation by adenosine and nitroglycerin in dogs. J Appl Physiol 76, 5 (1994), 1951-60.

[41] Hamilton, W. F., Moore, J. W., Kinsman, J. M., and Spurling, R. G. Simultaneous determination of the pulmonary and systemic circulation times in man and of a figure related to the cardiac output. Am J Physiol 84, 2 (1928), $338-344$.

[42] Hamilton, W. F., Moore, J. W., Kinsman, J. M., and Spurling, R. G. Studies on the circulation: IV. Further analysis of the injection method, and of changes in hemodynamics under physiological and pathological conditions. Am J Physiol 99, 3 (1932), 534-551.

[43] Hansson, G. K. Inflammation, atherosclerosis, and coronary artery disease. $N$ Engl J Med 352, 16 (2005), 1685-95.

[44] Heiss, H. W., Barmeyer, J., Wink, K., Hell, G., Cerny, F. J., Keul, J., AND Reindell, H. Studies on the regulation of myocardial blood flow in man. I.: Training effects on blood flow and metabolism of the healthy heart at rest and during standardized heavy exercise. Basic Res Cardiol 71, 6 (1976), $658-75$. 
[45] Henquell, L., And Honig, C. R. Intercapillary distances and capillary reserve in right and left ventricles: significance for control of tissue $\mathrm{pO}_{2}$. Microvasc Res 12, 1 (1976), 35-41.

[46] Henriksen, J. H., Moller, S., Fuglsang, S., and Bendtsen, F. Detection of early central circulatory transits in patients with cirrhosis by gamma variate fit of indicator dilution profiles. Am J Physiol Gastrointest Liver Physiol 288, 4 (2005), G677-84.

[47] Henry, C. B., And Duling, B. R. Permeation of the luminal capillary glycocalyx is determined by hyaluronan. Am J Physiol 27r, 2 Pt 2 (1999), H508-14.

[48] Henry, C. B., And Duling, B. R. TNF-alpha increases entry of macromolecules into luminal endothelial cell glycocalyx. Am J Physiol Heart Circ Physiol 279, 6 (2000), H2815-23.

[49] Hirche, H., And Lochner, W. [Measurement of the blood flow and blood filling of the coronary vascular bed with the test substance injection method on the anesthetized dog with closed thorax.]. Pflugers Arch Gesamte Physiol Menschen Tiere 274 (1962), 624-32.

[50] Hoeft, A., Schorn, B., Weyland, A., Scholz, M., Buhre, W., Stepanek, E., Allen, S. J., And Sonntag, H. Bedside assessment of intravascular volume status in patients undergoing coronary bypass surgery. Anesthesiology 81, 1 (1994), 76-86.

[51] Hoffman, E., And Ritman, E. Intramyocardial blood volume - implications for analysis of myocardial mechanical characteristics via in vivo imaging of the heart. Martinus Nujhoff Publishers, Dordrecht, 1987.

[52] Huxley, V. H., And Williams, D. A. Role of a glycocalyx on coronary arteriole permeability to proteins: evidence from enzyme treatments. Am J Physiol Heart Circ Physiol 278, 4 (2000), H1177-85.

[53] Ishibashi, Y., Duncker, D. J., Zhang, J., And Bache, R. J. ATP-sensitive $\mathrm{K}^{+}$channels, adenosine, and nitric oxide-mediated mechanisms account for coronary vasodilation during exercise. Circ Res 82, 3 (1998), 346-59. 
[54] Jacob, M., Bruegger, D., Rehm, M., Stoeckelhuber, M., Welsch, U., Conzen, P., And Becker, B. F. The endothelial glycocalyx affords compatibility of Starling's principle and high cardiac interstitial albumin levels. Cardiovasc Res 73, 3 (2007), 575-86.

[55] Jacob, M., Conzen, P., Finsterer, U., Krafft, A., Becker, B. F., AND REHM, M. Technical and physiological background of plasma volume measurement with indocyanine green: a clarification of misunderstandings. $J$ Appl Physiol 102, 3 (2007), 1235-42.

[56] JAmES, T. N. The chronotropic action of ATP and related compounds studied by direct perfusion of the sinus node. J Pharmacol Exp Ther 149, 2 (1965), 233-47.

[57] Jeremias, A., Whitbourn, R. J., Filardo, S. D., Fitzgerald, P. J., Cohen, D. J., Tuzcu, E. M., Anderson, W. D., Abizaid, A. A., Mintz, G. S., Yeung, A. C., Kern, M. J., And Yock, P. G. Adequacy of intracoronary versus intravenous adenosine-induced maximal coronary hyperemia for fractional flow reserve measurements. Am Heart J 140, 4 (2000), 651-7.

[58] Jones, C. J., Kuo, L., Davis, M. J., And Chilian, W. M. Regulation of coronary blood flow: coordination of heterogeneous control mechanisms in vascular microdomains. Cardiovasc Res 29, 5 (1995), 585-96.

[59] Kahler, E., Waller, C., Rommel, E., Belle, V., Hiller, K. H., Voll, S., Bauer, W. R., And HaAse, A. Perfusion-corrected mapping of cardiac regional blood volume in rats in vivo. Magn Reson Med 42, 3 (1999), 500-6.

[60] Kal, J. E., Van Wezel, H. B., And Vergroesen, I. A critical appraisal of the rate pressure product as index of myocardial oxygen consumption for the study of metabolic coronary flow regulation. Int J Cardiol 71, 2 (1999), 141-8.

[61] Kanatsuka, H., Lamping, K. G., Eastham, C. L., Dellsperger, K. C., And Marcus, M. L. Comparison of the effects of increased myocardial oxygen consumption and adenosine on the coronary microvascular resistance. Circ Res 65, 5 (1989), 1296-305. 
[62] Kaski, J. C. Pathophysiology and management of patients with chest pain and normal coronary arteriograms (cardiac syndrome X). Circulation 109, 5 (2004), $568-72$.

[63] Kassab, G. S., and Fung, Y. C. Topology and dimensions of pig coronary capillary network. Am J Physiol 267, 1 Pt 2 (1994), H319-25.

[64] Kassab, G. S., Lin, D. H., and Fung, Y. C. Morphometry of pig coronary venous system. Am J Physiol 267, 6 Pt 2 (1994), H2100-13.

[65] Kassab, G. S., Rider, C. A., Tang, N. J., and Fung, Y. C. Morphometry of pig coronary arterial trees. Am J Physiol 265, 1 Pt 2 (1993), H350-65.

[66] Kassell, N. F., Boarini, D. J., Olin, J. J., And Sprowell, J. A. Cerebral and systemic circulatory effects of arterial hypotension induced by adenosine. $J$ Neurosurg 58, 1 (1983), 69-76.

[67] Kaufmann, P. A., Rimoldi, O., Gnecchi-Ruscone, T., Bonser, R. S., Luscher, T. F., And Camici, P. G. Systemic inhibition of nitric oxide synthase unmasks neural constraint of maximal myocardial blood flow in humans. Circulation 110, 11 (2004), 1431-6.

[68] KaUL, S. The role of capillaries in determining coronary blood flow reserve: implications for stress-induced reversible perfusion defects. J Nucl Cardiol 8, 6 (2001), 694-700.

[69] Kaul, S. Microbubbles and ultrasound: a bird's eye view. Trans Am Clin Climatol Assoc 115 (2004), 137-48; discussion 148.

[70] Kaul, S. Evaluating the 'no reflow' phenomenon with myocardial contrast echocardiography. Basic Res Cardiol 101, 5 (2006), 391-9.

[71] Khouri, E. M., Gregg, D. E., and Rayford, C. R. Effect of exercise on cardiac output, left coronary flow and myocardial metabolism in the unanesthetized dog. Circ Res 17, 5 (1965), 427-37.

[72] Kitamura, K., Jorgensen, C. R., Gobel, F. L., Taylor, H. L., and WANG, Y. Hemodynamic correlates of myocardial oxygen consumption during upright exercise. J Appl Physiol 32, 4 (1972), 516-22. 
[73] Kiyooka, T., Hiramatsu, O., Shigeto, F., Nakamoto, H., Tachibana, H., Yada, T., Ogasawara, Y., Kajiya, M., Morimoto, T., Morizane, Y., Mohri, S., Shimizu, J., Ohe, T., and KaJiya, F. Direct observation of epicardial coronary capillary hemodynamics during reactive hyperemia and during adenosine administration by intravital video microscopy. Am J Physiol Heart Circ Physiol 288, 3 (2005), H1437-43.

[74] Klitzman, B., And Duling, B. R. Microvascular hematocrit and red cell flow in resting and contracting striated muscle. Am J Physiol 237, 4 (1979), H481-90.

[75] Knopp, T. J., Dobbs, W. A., Greenleaf, J. F., And Bassingthwaighte, J. B. Transcoronary intravascular transport functions obtained via a stable deconvolution technique. Ann Biomed Eng 4, 1 (1976), 44-59.

[76] Lehr, H. A., And Messmer, K. The microcirculation in atherogenesis. Cardiovasc Res 32, 4 (1996), 781-8.

[77] Li, X., Tian, J., And Millard, R. K. Erroneous and inappropriate use of gamma fits to tracer-dilution curves in magnetic resonance imaging and nuclear medicine. Magn Reson Imaging 21, 9 (2003), 1095-6.

[78] LiBBy, P. Inflammation in atherosclerosis. Nature 420, 6917 (2002), 868-74.

[79] Lipowsky, H. H. Microvascular rheology and hemodynamics. Microcirculation 12, $1(2005), 5-15$.

[80] Ljungstrom, K. G. Dextran 40 therapy made safer by pretreatment with dextran 1. Plast Reconstr Surg 120, 1 (2007), 337-40.

[81] Madsen, M. T. A simplified formulation of the gamma variate function. Physics in Medicine and Biology, 7 (1992), 1597.

[82] Massey, E. J., de Souza, P., Findlay, G., Smithies, M., Shah, S., Spark, P., Newcombe, R. G., Phillips, C., Wardrop, C. A., and RobinsOn, G. T. Clinically practical blood volume assessment with fluoresceinlabeled HES. Transfusion 44, 2 (2004), 151-7. 
[83] Megens, R. T., Reitsma, S., Schiffers, P. H., Hilgers, R. H., De Mey, J. G., Slaaf, D. W., oude Egbrink, M. G., And van Zandvoort, M. A. Two-photon microscopy of vital murine elastic and muscular arteries. Combined structural and functional imaging with subcellular resolution. J Vasc Res 44, 2 (2007), 87-98.

[84] Meier, P., AND Zierler, K. L. On the theory of the indicator-dilution method for measurement of blood flow and volume. J Appl Physiol 6, 12 (1954), $731-44$.

[85] Merkus, D., Haitsma, D. B., Fung, T. Y., Assen, Y. J., Verdouw, P. D., AND Duncker, D. J. Coronary blood flow regulation in exercising swine involves parallel rather than redundant vasodilator pathways. Am J Physiol Heart Circ Physiol 285, 1 (2003), H424-33.

[86] Michel, C. C., And Curry, F. R. Glycocalyx volume: a critical review of tracer dilution methods for its measurement. Microcirculation 16, 3 (2009), 213-9.

[87] Millard, R. K. Indicator-dilution dispersion models and cardiac output computing methods. Am J Physiol 272, 4 Pt 2 (1997), H2004-12.

[88] Mischi, M., Den Boer, J. A., And Korsten, H. H. On the physical and stochastic representation of an indicator dilution curve as a gamma variate. Physiol Meas 29, 3 (2008), 281-94.

[89] Mischi, M., Kalker, T., And Korsten, H. H. Videodensitometric methods for cardiac output measurements. EURASIP J. Appl. Signal Process. 2003 (2003), 479-489.

[90] Mochizuki, S., Vink, H., Hiramatsu, O., Kajita, T., Shigeto, F., SpaAn, J. A., AND KAJIYA, F. Role of hyaluronic acid glycosaminoglycans in shear-induced endothelium-derived nitric oxide release. Am J Physiol Heart Circ Physiol 285, 2 (2003), H722-6.

[91] Morgenstern, C., Holjes, U., Arnold, G., And Lochner, W. The influence of coronary pressure and coronary flow on intracoronary blood volume and geometry of the left ventricle. Pflugers Arch 340, 2 (1973), 101-11. 
[92] Mulivor, A. W., And Lipowsky, H. H. Role of glycocalyx in leukocyteendothelial cell adhesion. Am J Physiol Heart Circ Physiol 283, 4 (2002), H128291.

[93] Mulivor, A. W., And Lipowsky, H. H. Inflammation- and ischemia-induced shedding of venular glycocalyx. Am J Physiol Heart Circ Physiol 286, 5 (2004), H1672-80.

[94] Muniyappa, R., Montagnani, M., Koh, K. K., and Quon, M. J. Cardiovascular actions of insulin. Endocr Rev 28, 5 (2007), 463-91.

[95] Munoz, E. M., And Linhardt, R. J. Heparin-binding domains in vascular biology. Arterioscler Thromb Vasc Biol 24, 9 (2004), 1549-57.

[96] Murdock, R. C., Reynolds, C., Sarelius, I. H., and Waugh, R. E. Adaptation and survival of surface-deprived red blood cells in mice. Am J Physiol Cell Physiol 279, 4 (2000), C970-80.

[97] Nelson, R. R., Gobel, F. L., Jorgensen, C. R., Wang, K., Wang, Y., AND TAYLOR, H. L. Hemodynamic predictors of myocardial oxygen consumption during static and dynamic exercise. Circulation 50, 6 (1974), 1179-89.

[98] Netter, F. H. Atlas of Human Anatomy, 2nd ed. Rittenhouse Book Distributors Inc., 1997.

[99] Nieuwdorp, M., Holleman, F., de Groot, E., Vink, H., Gort, J., Kontush, A., Chapman, M. J., Hutten, B. A., Brouwer, C. B., Hoekstra, J. B., Kastelein, J. J., And Stroes, E. S. Perturbation of hyaluronan metabolism predisposes patients with type 1 diabetes mellitus to atherosclerosis. Diabetologia 50, 6 (2007), 1288-93.

[100] Nieuwdorp, M., Meuwese, M. C., Mooij, H. L., Ince, C., Broekhuizen, L. N., Kastelein, J. J., Stroes, E. S., And Vink, H. Measuring endothelial glycocalyx dimensions in humans: a potential novel tool to monitor vascular vulnerability. J Appl Physiol 104, 3 (2008), 845-52.

[101] Nieuwdorp, M., Meuwese, M. C., Mooij, H. L., van Lieshout, M. H., Hayden, A., Levi, M., Meijers, J. C., Ince, C., Kastelein, J. J., Vink, 
H., And Stroes, E. S. Tumor necrosis factor-alpha inhibition protects against endotoxin-induced endothelial glycocalyx perturbation. Atherosclerosis (2008).

[102] Nieuwdorp, M., Meuwese, M. C., Vink, H., Hoekstra, J. B., Kastelein, J. J., And Stroes, E. S. The endothelial glycocalyx: a potential barrier between health and vascular disease. Curr Opin Lipidol 16, 5 (2005), 507-11.

[103] Nieuwdorp, M., Mooij, H. L., Kroon, J., Atasever, B., Spaan, J. A., Ince, C., Holleman, F., Diamant, M., Heine, R. J., Hoekstra, J. B., Kastelein, J. J., Stroes, E. S., And Vink, H. Endothelial glycocalyx damage coincides with microalbuminuria in type 1 diabetes. Diabetes 55, 4 (2006), 1127-32.

[104] Nieuwdorp, M., van Haeften, T. W., Gouverneur, M. C., Mooij, H. L., van Lieshout, M. H., Levi, M., Meijers, J. C., Holleman, F., Hoekstra, J. B., Vink, H., Kastelein, J. J., And Stroes, E. S. Loss of endothelial glycocalyx during acute hyperglycemia coincides with endothelial dysfunction and coagulation activation in vivo. Diabetes 55, 2 (2006), 480-6.

[105] O’Neill, P. G., Charlat, M. L., Michael, L. H., Roberts, R., and Bolli, R. Influence of neutrophil depletion on myocardial function and flow after reversible ischemia. Am J Physiol 256, 2 Pt 2 (1989), H341-51.

[106] Orth, V. H., Rehm, M., Thiel, M., Kreimeier, U., Haller, M., Brechtelsbauer, H., And Finsterer, U. First clinical implications of perioperative red cell volume measurement with a nonradioactive marker (sodium fluorescein). Anesth Analg 87, 6 (1998), 1234-8.

[107] Pahakis, M. Y., Kosky, J. R., Dull, R. O., and Tarbell, J. M. The role of endothelial glycocalyx components in mechanotransduction of fluid shear stress. Biochem Biophys Res Commun 355, 1 (2007), 228-33.

[108] Park, S. M., Shim, W. J., Song, W. H., Lim, D. S., Kim, Y. H., And Ro, Y. M. Effects of smoking on coronary blood flow velocity and coronary flow reserve assessed by transthoracic Doppler echocardiography. Echocardiography 23, 6 (2006), 465-70. 
[109] Pijls, N. H., van Schanrdenburgh, P., Manoharan, G., Boersma, E., Bech, J. W., Van't Veer, M., Bar, F., Hoorntje, J., Koolen, J., WiJns, W., ANd DE BRuYne, B. Percutaneous coronary intervention of functionally nonsignificant stenosis: 5-year follow-up of the DEFER Study. J Am Coll Cardiol 49, 21 (2007), 2105-11.

[110] Platts, S. H., And Duling, B. R. Adenosine A3 receptor activation modulates the capillary endothelial glycocalyx. Circ Res 94, 1 (2004), 77-82.

[111] Platts, S. H., Linden, J., And Duling, B. R. Rapid modification of the glycocalyx caused by ischemia-reperfusion is inhibited by adenosine A2A receptor activation. Am J Physiol Heart Circ Physiol 284, 6 (2003), H2360-7.

[112] Pries, A. R., Ley, K., And Gaehtgens, P. Generalization of the Fahraeus principle for microvessel networks. Am J Physiol 251, 6 Pt 2 (1986), H1324-32.

[113] Pries, A. R., And Secomb, T. W. Microvascular blood viscosity in vivo and the endothelial surface layer. Am J Physiol Heart Circ Physiol 289, 6 (2005), H2657-64.

[114] Pries, A. R., Secomb, T. W., And Gaentgens, P. The endothelial surface layer. Pflugers Arch 440, 5 (2000), 653-66.

[115] Pries, A. R., Secomb, T. W., Jacobs, H., Sperandio, M., Osterloh, K., And Gaehtgens, P. Microvascular blood flow resistance: role of endothelial surface layer. Am J Physiol 273, 5 Pt 2 (1997), H2272-9.

[116] Rehm, M., Bruegger, D., Christ, F., Conzen, P., Thiel, M., Jacob, M., Chappell, D., Stoeckelhuber, M., Welsch, U., Reichart, B., Peter, K., AND BECKER, B. F. Shedding of the endothelial glycocalyx in patients undergoing major vascular surgery with global and regional ischemia. Circulation 116, 17 (2007), 1896-906.

[117] Reitsma, S., Slaaf, D. W., Vink, H., van Zandvoort, M. A., and oude EgBrink, M. G. The endothelial glycocalyx: composition, functions, and visualization. Pflugers Arch 454, 3 (2007), 345-59. 
[118] Rizzo, V., McIntosh, D. P., Oh, P., And Schnitzer, J. E. In situ flow activates endothelial nitric oxide synthase in luminal caveolae of endothelium with rapid caveolin dissociation and calmodulin association. J Biol Chem 273, 52 (1998), 34724-9.

[119] Rosenberg, R. D., Shworak, N. W., Liu, J., Schwartz, J. J., And ZHANG, L. Heparan sulfate proteoglycans of the cardiovascular system. Specific structures emerge but how is synthesis regulated? J Clin Invest 99, 9 (1997), $2062-70$.

[120] Rowe, G. G., Afonso, S., Gurtner, H. P., Chelius, C. J., Lowe, W. C., Castillo, C. A., and Crumpton, C. W. The systemic and coronary hemodynamic effects of adenosine triphosphate and adenosine. Am Heart J 64 (1962), $228-34$.

[121] Rubio-Gayosso, I., Platts, S. H., And Duling, B. R. Reactive oxygen species mediate modification of glycocalyx during ischemia-reperfusion injury. Am J Physiol Heart Circ Physiol 290, 6 (2006), H2247-56.

[122] Salisbury, P. F., Cross, C. E., And Rieben, P. A. Physiological factors influencing coronary blood volume in isolated dog hearts. Am J Physiol 200 (1961), 633-6.

[123] Sarelius, I. H., Cohen, K. D., And Murrant, C. L. Role for capillaries in coupling blood flow with metabolism. Clin Exp Pharmacol Physiol 2\%, 10 (2000), 826-9.

[124] Sarelius, I. H., And Duling, B. R. Direct measurement of microvessel hematocrit, red cell flux, velocity, and transit time. Am J Physiol 243, 6 (1982), H1018-26.

[125] Savery, M. D., And Damiano, E. R. The endothelial glycocalyx is hydrodynamically relevant in arterioles throughout the cardiac cycle. Biophys $J$ (2008).

[126] Secomb, T. W., Hsu, R., And Pries, A. R. A model for red blood cell motion in glycocalyx-lined capillaries. Am J Physiol 274, 3 Pt 2 (1998), H1016-22. 
[127] Secomb, T. W., Hsu, R., And Pries, A. R. Motion of red blood cells in a capillary with an endothelial surface layer: effect of flow velocity. Am J Physiol Heart Circ Physiol 281, 2 (2001), H629-36.

[128] Siegel, G., Malmsten, M., Klussendorf, D., Walter, A., Schnalke, F., And Kauschmann, A. Blood-flow sensing by anionic biopolymers. J Auton Nerv Syst 57, 3 (1996), 207-13.

[129] Smith, M. L., Long, D. S., Damiano, E. R., And Ley, K. Near-wall microPIV reveals a hydrodynamically relevant endothelial surface layer in venules in vivo. Biophys J 85, 1 (2003), 637-45.

[130] SpaAn, J. A. Coronary diastolic pressure-flow relation and zero flow pressure explained on the basis of intramyocardial compliance. Circ Res 56, 3 (1985), $293-309$.

[131] Spaan, J. A., Breuls, N. P., And Laird, J. D. Forward coronary flow normally seen in systole is the result of both forward and concealed back flow. Basic Res Cardiol 76, 5 (1981), 582-6.

[132] Stamler, J. S., Jaraki, O., Osborne, J., Simon, D. I., Keaney, J., Vita, J., Singel, D., Valeri, C. R., and Loscalzo, J. Nitric oxide circulates in mammalian plasma primarily as an S-nitroso adduct of serum albumin. Proc Natl Acad Sci U S A 89, 16 (1992), 7674-7.

[133] Stokes, K. Y., And Granger, D. N. The microcirculation: a motor for the systemic inflammatory response and large vessel disease induced by hypercholesterolaemia? J Physiol 562, Pt 3 (2005), 647-53.

[134] Tansley, P., Yacoub, M., Rimoldi, O., Birks, E., Hardy, J., Hipkin, M., Bowles, C., Kindler, H., Dutka, D., And Camici, P. G. Effect of left ventricular assist device combination therapy on myocardial blood flow in patients with end-stage dilated cardiomyopathy. J Heart Lung Transplant 23, 11 (2004), 1283-9.

[135] Tarbell, J. M., And Ebong, E. E. The endothelial glycocalyx: a mechanosensor and -transducer. Sci Signal 1, 40 (2008), pt8. 
[136] Tarbell, J. M., And Pahakis, M. Y. Mechanotransduction and the glycocalyx. J Intern Med 259, 4 (2006), 339-50.

[137] Thompson, H. K., J., Starmer, C. F., Whalen, R. E., and McIntosh, H. D. Indicator transit time considered as a gamma variate. Circ Res 14 (1964), $502-15$.

[138] Tune, J. D., Gorman, M. W., And Feigl, E. O. Matching coronary blood flow to myocardial oxygen consumption. J Appl Physiol 97, 1 (2004), 404-15.

[139] Tune, J. D., Richmond, K. N., Gorman, M. W., and Feigl, E. O. Control of coronary blood flow during exercise. Exp Biol Med (Maywood) 227, 4 (2002), 238-50.

[140] Tyml, K., And Cheng, L. Heterogeneity of red blood cell velocity in skeletal muscle decreases with increased flow. Microcirculation 2, 2 (1995), 181-93.

[141] van den Berg, B. M., Spaan, J. A., Rolf, T. M., And Vink, H. Atherogenic region and diet diminish glycocalyx dimension and increase intima-tomedia ratios at murine carotid artery bifurcation. Am J Physiol Heart Circ Physiol 290, 2 (2006), H915-20.

[142] VAn Den Berg, B. M., Vink, H., And SpaAn, J. A. The endothelial glycocalyx protects against myocardial edema. Circ Res 92, 6 (2003), 592-4.

[143] van der Ploeg, C. P., Dankelman, J., And Spann, J. A. Functional distribution of coronary vascular volume in beating goat hearts. Am J Physiol 264, 3 Pt 2 (1993), H770-6.

[144] van Haaren, P. M., VanBavel, E., Vink, H., and Spaan, J. A. Localization of the permeability barrier to solutes in isolated arteries by confocal microscopy. Am J Physiol Heart Circ Physiol 285, 6 (2003), H2848-56.

[145] van Haaren, P. M., VanBavel, E., Vink, H., and Spaan, J. A. Charge modification of the endothelial surface layer modulates the permeability barrier of isolated rat mesenteric small arteries. Am J Physiol Heart Circ Physiol 289, 6 (2005), H2503-7. 
[146] van Kreel, B. K., van Beek, E., Spaanderman, M. E., and Peeters, L. L. A new method for plasma volume measurements with unlabeled dextran70 instead of 125I-labeled albumin as an indicator. Clin Chim Acta 275, 1 (1998), 71-80.

[147] van Vlijmen, B. J., van den Maagdenberg, A. M., Gijbels, M. J., van der Boom, H., HogenEsch, H., Frants, R. R., Hofker, M. H., And Havekes, L. M. Diet-induced hyperlipoproteinemia and atherosclerosis in apolipoprotein E3-Leiden transgenic mice. J Clin Invest 93, 4 (1994), 140310 .

[148] van Wezel, H. B., Kal, J. E., Vergroesen, I., Vroom, M. B., De Graaf, R., Dankelman, J., Porsius, M., and Spann, J. A. Rate of coronary flow adaptation in response to changes in heart rate before and during anesthesia for coronary artery surgery. Anesthesiology 84, 5 (1996), 1107-18.

[149] VanTeeffelen, J. W. How to prevent leaky vessels during reperfusion? Just keep that glycocalyx sealant in place! Critical Care 12, 4 (2008).

[150] Vanteeffelen, J. W., Brands, J., Jansen, C., Spaan, J. A., And Vink, H. Heparin impairs glycocalyx barrier properties and attenuates shear dependent vasodilation in mice. Hypertension 50, 1 (2007), 261-7.

[151] Vanteeffelen, J. W., Brands, J., Stroes, E. S., And Vink, H. Endothelial glycocalyx: Sweet shield of blood vessels. Trends in Cardiovascular Medicine 17, 3 (2007), 101-105.

[152] Vanteeffelen, J. W., Constantinescu, A., Brands, J., Spaan, J. A., AND VINK, H. Bradykinin- and sodium nitroprusside-induced increases in capillary tube haematocrit in mouse cremaster muscle are associated with impaired glycocalyx barrier properties. J Physiol (2008).

[153] Vanteeffelen, J. W., Constantinescu, A. A., Vink, H., And Spaan, J. A. Hypercholesterolemia impairs reactive hyperemic vasodilation of $2 \mathrm{~A}$ but not 3A arterioles in mouse cremaster muscle. Am J Physiol Heart Circ Physiol 289, 1 (2005), H447-54. 
[154] Vanteeffelen, J. W., Dekker, S., Fokkema, D. S., Siebes, M., Vink, H., AND SpAAn, J. A. Hyaluronidase treatment of coronary glycocalyx increases reactive hyperemia but not adenosine hyperemia in $\operatorname{dog}$ hearts. Am J Physiol Heart Circ Physiol 289, 6 (2005), H2508-13.

[155] Vilar, R. E., Ghael, D., Li, M., Bhagat, D. D., Arrigo, L. M., Cowman, M. K., Dweck, H. S., And Rosenfeld, L. Nitric oxide degradation of heparin and heparan sulphate. Biochem J 324 ( Pt 2) (1997), 473-9.

[156] Vink, H., Constantinescu, A. A., And Spaan, J. A. Oxidized lipoproteins degrade the endothelial surface layer : implications for platelet-endothelial cell adhesion. Circulation 101, 13 (2000), 1500-2.

[157] Vink, H., And Duling, B. R. Identification of distinct luminal domains for macromolecules, erythrocytes, and leukocytes within mammalian capillaries. Circ Res 79, 3 (1996), 581-9.

[158] Vink, H., And Duling, B. R. Capillary endothelial surface layer selectively reduces plasma solute distribution volume. Am J Physiol Heart Circ Physiol 278, 1 (2000), H285-9.

[159] Vink, H., Stace, T. M., and Damiano, E. R. High resolution 3D intravital fluorescence microscopy reveals partial exclusion of anionic tracers within a 1 micron thick capillary endothelial cell glycocalyx. Faseb Journal 17, 4 (2003), A70-A70.

[160] von Restorff, W., Holtz, J., And Bassenge, E. Exercise induced augmentation of myocardial oxygen extraction in spite of normal coronary dilatory capacity in dogs. Pflugers Arch 372, 2 (1977), 181-5.

[161] Waller, C., Kahler, E., Hiller, K. H., Hu, K., Nahrendorf, M., Voll, S., HaAse, A., Ertl, G., And Bauer, W. R. Myocardial perfusion and intracapillary blood volume in rats at rest and with coronary dilatation: MR imaging in vivo with use of a spin-labeling technique. Radiology 215, 1 (2000), $189-97$. 
[162] Weinbaum, S., Tarbell, J. M., and Damiano, E. R. The structure and function of the endothelial glycocalyx layer. Annu Rev Biomed Eng 9 (2007), $121-67$.

[163] Weinbaum, S., Zhang, X., Han, Y., Vink, H., and Cowin, S. C. Mechanotransduction and flow across the endothelial glycocalyx. Proc Natl Acad Sci U $S$ A 100, 13 (2003), 7988-95.

[164] Wilson, R. F., Wyche, K., Christensen, B. V., Zimmer, S., and LaxSON, D. D. Effects of adenosine on human coronary arterial circulation. Circulation 82, 5 (1990), 1595-606.

[165] Wolf, R. A., Chaung, L. Y., O’Hara, D., Smith, T. W., and Muller, J. E. The serum kinetics of bovine testicular hyaluronidase in dogs, rats and humans. J Pharmacol Exp Ther 222, 2 (1982), 331-7.

[166] Yao, Y., Rabodzey, A., And Dewey, C. F., J. Glycocalyx modulates the motility and proliferative response of vascular endothelium to fluid shear stress. Am J Physiol Heart Circ Physiol 293, 2 (2007), H1023-30.

[167] Yusa, T., And Obara, S. Myocardial oxygen extraction rate under general anesthesia. Tohoku J Exp Med 133, 3 (1981), 321-4.

[168] Ziegler, W. H., And Goresky, C. A. Transcapillary exchange in the working left ventricle of the dog. Circ Res 29, 2 (1971), 181-207.

[169] ZIERLER, K. Indicator dilution methods for measuring blood flow, volume, and other properties of biological systems: a brief history and memoir. Ann Biomed Eng 28, 8 (2000), 836-48.

[170] ZierLER, K. L. Theoretical basis of indicator-dilution methods for measuring flow and volume. Circ Res 10, 3 (1962), 393-407.

[171] Zutphen, L. F. M. V., Baumans, V., And Beynen, A., Eds. Handboek proefdierkunde proefdieren, dierproeven, alternatieven en ethiek, vierde druk ed. Elsevier gezondheidszorg, Maarssen, 2003. 
[172] Zuurbier, C. J., Demirci, C., Koeman, A., Vink, H., And Ince, C. Shortterm hyperglycemia increases endothelial glycocalyx permeability and acutely decreases lineal density of capillaries with flowing red blood cells. J Appl Physiol 99, 4 (2005), 1471-6. 
Summary

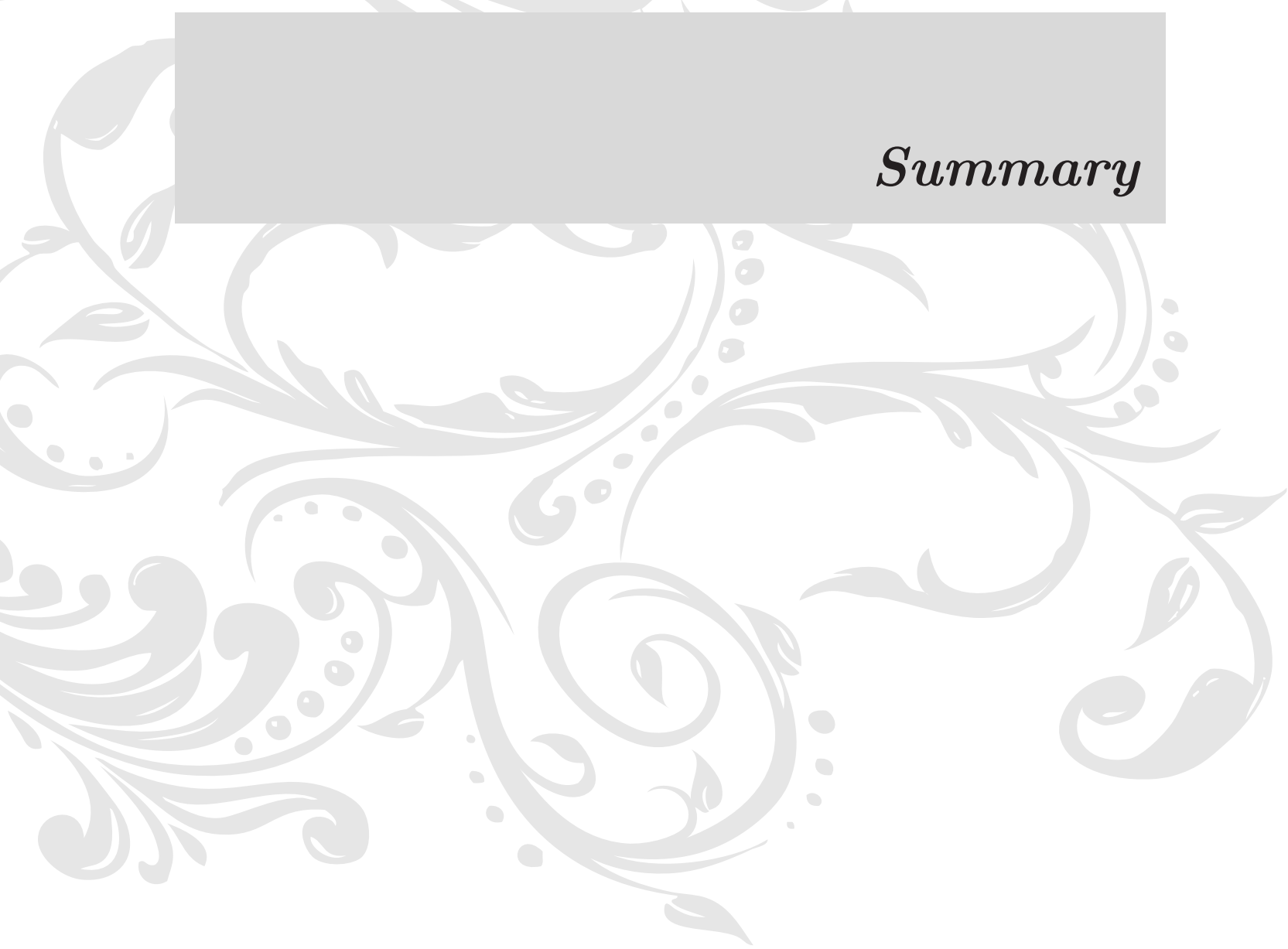




\section{Agonist induced modulation of the endothelial glycocalyx}

The endothelial glycocalyx profoundly reduces functionally perfused capillary volume by being inaccessible for flowing blood. Because the accessibility of the glycocalyx for flowing plasma was suggested to be increased by adenosine and metabolic stimuli, the aim of our project was to investigate whether agonist-induced glycocalyx modulation is occurring in the systemic and coronary circulation and whether this might be an additional mechanism by which vasoactive substances can contribute to flow and volume regulation, as well as the exchange capacity in the heart.

Systemic blood volume was measured using labeled red blood cells. Total blood volume was defined as the sum of red blood cell and red blood cell derived plasma volume using large vessel hematocrit. Systemic glycocalyx volume was determined from the difference in distribution volume of circulating plasma and the distribution volume of dextrans with a molecular weight of $40 \mathrm{kDa}$. Volume measurements were done at baseline and during intravenous administration of adenosine $(157 \pm 11.6 \mu \mathrm{g} / \mathrm{kg} / \mathrm{min})$. In the systemic circulation of goats it was observed that during intravenous administration of adenosine the difference between a glycocalyx permeable and impermeable tracer was reduced from $23.9 \pm 5.3(\mathrm{SEM}) \mathrm{ml} / \mathrm{kg}$ bodyweight $(\mathrm{BW})$ to $3.3 \pm 3.2 \mathrm{ml} / \mathrm{kg}$ BW $(\mathrm{P}<0.05)$, illustrating that adenosine indeed has the potency to greatly increase glycocalyx accessibility for flowing blood. The reduction in glycocalyx volume was not accompanied with an increase in plasma or blood volume, but appeared to be due to a reduction in anatomic vascular volume.

Coronary blood volume was determined using the indicator dilution technique. Total coronary blood volume was defined as the sum of red blood cell volume and the distribution volume of an assumed plasma tracer (dextrans with a molecular weight of 2000 $\mathrm{kDa}$ ). After comparing the suitability of four models, the local density random walk model was chosen to analyze the measured data. Volume measurements were done at baseline and during intracoronary adenosine administration $(0.2-0.6 \mathrm{mg} / \mathrm{kg} / \mathrm{h})$ in the presence of an intact glycocalyx and after enzymatic degradation using the en- 
zyme hyaluronidase (170.000 Units). Coronary blood flow was measured continuously by having a flowprobe around the left main coronary artery. Coronary adenosine infusion resulted in a profound recruitment of coronary blood volume; it increased significantly from $18.9 \pm 1.1 \mathrm{ml} / 100 \mathrm{gr}$ heart tissue under baseline conditions to 33.2 $\pm 5.3 \mathrm{ml} / 100 \mathrm{gr}$ during adenosine. After hyaluronidase, the blood volume increased to $26.3 \pm 2.7 \mathrm{ml} / 100 \mathrm{gr}$, without an effect on coronary blood flow. Infusion of adenosine after hyaluronidase increased the blood volume to $33.9 \pm 6.8 \mathrm{ml} / 100 \mathrm{gr}$, illustrating impairment of adenosine-induced recruitment of coronary blood volume in case of glycocalyx loss. The increase in volume during hyaluronidase without a change in coronary blood flow and the increase in coronary blood flow during adenosine after hyaluronidase with a limited increase in coronary blood volume suggest that flow reserve and volume recruitment are, to some extent, two independent processes. Measurement of coronary flow reserve with adenosine, as done in the clinic, is therefore not sensitive to glycocalyx damage.

Coronary oxygen exchange capacity was determined from simultaneously taken arterial and coronary venous blood samples and coronary blood flow. Arterial and coronary venous blood samples were taken at heart rates of 80, 110, 140 and 170 beats per minute in the presence and absence (by hyaluronidase) of endothelial glycocalyx. When studying the exchange capacity in the heart a loss of blood excluding glycocalyx volume was associated with an increased oxygen exchange. The myocardial arteriovenous oxygen difference increased significantly from $7.0 \pm 0.2$ to $8.3 \pm 0.3 \mathrm{ml} / 100 \mathrm{ml}$, while no change in myocardial arteriovenous oxygen difference was observed in the control group. These data suggest that the presence of endothelial glycocalyx limits the myocardial oxygen exchange capacity under baseline conditions.

Thus, (1) adenosine administration results in a decrease in blood inaccessible glycocalyx volume; (2) loss of glycocalyx reduced volume recruitment capacity without having an effect on flow reserve, and (3) recruitment of glycocalyx volume increases oxygen extraction. 

Samenvatting 


\section{Agonist-geïnduceerde modulatie van de endotheliale glycocalyx}

\section{Achtergrond}

Adenosine is een vaatverwijdende stof die in de kliniek wordt gebruikt om de ernst van een stenose (vernauwing) in een kransslagader op de doorbloeding van het hart en de bijdrage aan de pijn op de borst te bepalen. Tijdens de toediening van adenosine wordt er gekeken wat de toename in bloedstroom in de kransslagader is. Hoe minder de toename in bloedstroom is, des te erger is het effect van de stenose. Er is echter een groep personen met pijn op de borst die geen noemenswaardige stenose heeft, of een vernauwing die geen relevant effect heeft op de toename in bloedstroom in de kransslagader tijdens adenosine. Er wordt gedacht dat er in deze groepen personen problemen zijn met de microvasculatuur, de kleinere vaten van het hart. Een mogelijke oorzaak van problemen met deze kleine vaten is schade aan de glycocalyx, een gelachtige laag van langgerekte suikermoleculen en gebonden plasma eiwitten. Deze laag is in principe ontoegankelijk voor stromend bloed en beslaat in de haarvaten (de allerkleinste vaten) een groot deel van het totale volume van het vat (het anatomisch volume). Het anatomisch volume van een vat is daardoor aanzienlijk groter dan het volume van het vat waar zich het stromende bloed bevindt (functionele bloed volume), zie figuur 1. De glycocalyx heeft voornamelijk een beschermende functie van de vaatwand en een afname van het glycocalyxvolume wordt dan ook geassocieerd met verschillende vaatwandproblemen, zoals verhoogde lekkage en oedeemvorming, een verstoorde vaatverwijding als reactie op een toename in bloedstroom, alsmede een toename van het aantal witte bloedcellen die blijven plakken aan de vaatwand. Het hebben van een intacte glycocalyx lijkt dus van belang voor het hebben van gezonde bloedvaten. In patiënten met klachten maar zonder noemenswaardige stenose zou het meten van het glycocalyxvolume een goede alternatieve methode kunnen zijn om te bepalen of deze personen een risico lopen op hartproblemen. 


\section{"De glycocalyx is een soort anti-aanbak laag in je bloedvaten, zolang deze er zit blijft er niks plak- ken"}

De glycocalyx zit in ieder bloedvat maar neemt in kleinere vaten relatief meer volume in. Het klassieke idee in de tekstboeken is dat de kleinste vaten, de haarvaten, starre buisjes zijn en dat de perfusie geregeld wordt door de weerstandsvaten. Door het opengaan van de weerstandsvaten gaat de bloedstroom en het bloedvolume omhoog. Door de aanwezigheid van een relatief dikke laag glycocalyx in de haarvaten is er een andere optie mogelijk; een toename in bloedvolume door veranderingen in de eigenschappen van de glycocalyx (toegankelijk worden voor stromend bloed) waardoor het bloedvolume in deze vaten toeneemt, zie figuur 1. Een toename in volume door verandering van de glycocalyx heeft tot gevolg dat de bijdrage van de weerstandsvaten aan de toename in bloedvolume minder is dan wat nu wordt gedacht en verandert de kijk op hoe de doorbloeding in het hart geregeld is. In eerdere studies was al opgemerkt dat de eigenschappen van de glycocalyx kunnen veranderen tijdens de toediening van adenosine. Adenosine wordt, zoals hierboven vermeld, in de kliniek gebruikt als vaatverwijder maar wordt ook door het hart zelf geproduceerd, bijvoorbeeld tijdens inspanning. Tot dusver was het effect van adenosine op de glycocalyx niet direct gemeten noch was de relevantie voor het hart ervan aangetoond. Het doel van onze studie was dan ook om te bepalen of de toename in bloedvolume tijdens de toediening van adenosine voor een deel bestaat uit het toegankelijk worden van glycocalyxvolume voor bloed. Verder waren we geïnteresseerd of de toename in bloedstroom tijdens adenosine beïnvloed wordt door de aanwezigheid van de glycocalyx. Ofwel kan schade aan de glycocalyx opgemerkt worden aan de hand van bloedstroommetingen? Als laatste hebben we gekeken naar de zuurstofuitwisseling in het hart. De glycocalyx bevindt zich met name in de haarvaten, de vaten waar de uitwisseling van zuustof, voedingstoffen en afvalstoffen plaatsvindt. Een toename in toegankelijkheid van bloed in de glycocalyx heeft dus wellicht gevolgen voor de uitwisseling van zuurstof. 



Figuur 1 Toename in bloedvolume door toename in toegankelijkheid voor bloed in de glycocalyx. Links is een vat te zien met glycocalyx en het bloed bevindt zich alleen daar in het vat waar geen glycocalyx is. Rechts is eenzelfde vat te zien maar nu zonder glycocalyx. Door de afwezigheid van glycocalyx is het volume toegankelijk voor bloed aanzienlijk groter. Het bloedvolume rechts is dus toegenomen ten opzichte van het vat links zonder dat de diameter van het vat veranderd is.

\section{Lichaamsniveau}

\section{Heeft adenosine een effect op het glycocalyxvolume in het li- chaam?}

Allereerst hebben we gekeken of adenosine een effect heeft op het glycocalyxvolume op lichaamsniveau. Om het glycocalyxvolume te meten werd het anatomisch volume (van wand tot wand) en het functioneel bloedvolume (alleen daar waar bloed zit) gemeten. Het verschil tussen deze twee volumes is het glycocalyxvolume. Deze meting werd gedaan in rust en tijdens de toediening van adenosine. Onder normale omstandigheden was er een groot verschil tussen het anatomisch en functioneel bloedvolume ( $\sim 500 \mathrm{ml})$. Tijdens adenosine, daarentegen, was het verschil afgenomen tot vrijwel nul. Deze studie toont aan dat bijna het gehele glycocalyxvolume tijdens adenosine toegankelijk is geworden voor stromend bloed. 
Samenvatting

\section{Glycocalyxvolume in het hart}

\section{Wat is de bijdrage van de glycocalyx aan de toename van bloed- volume in de kransvaten tijdens adenosine toediening?}

Hierna zijn we gaan kijken naar het effect van adenosine op het glycocalyxvolume in een specifiek orgaan, het hart. Ditmaal kon er niet gekeken worden naar het verschil tussen het anatomische volume en functioneel bloedvolume. Om de bijdrage van de glycocalyx aan de toename in bloedvolume tijdens adenosine te bepalen, is het bloedvolume in de vaten van het hart gemeten in rust en tijdens de toediening van adenosine. De metingen zijn gedaan met een intacte glycocalyx en herhaald na toediening van het enzym hyaluronidase dat de glycocalyx afbreekt. Met een intacte glycocalyx nam het coronaire bloedvolume toe van ongeveer $19 \mathrm{ml}$ per 100 gram hartgewicht naar 33 ml per 100 gram tijdens adenosine. Ook was er een toename in bloedstroom, deze werd 2.3 keer zo hoog als in rust. Het effect van adenosine is schematisch weergegeven in figuur 2. Na toediening van hyaluronidase was het bloedvolume in rust toegenomen tot circa $26 \mathrm{ml}$ per 100 gram en nam het tijdens adenosine verder toe tot bijna $34 \mathrm{ml}$ per 100 gram. De toename in volume tijdens adenosine na hyaluronidase was dus aanzienlijk minder (ongeveer de helft) dan bij de meting met een intacte glycocalyx. Dit komt doordat het bloedvolume na hyaluronidase al was toegenomen. Het bloedvolume tijdens adenosine met en zonder glycocalyx was gelijk wat betekent dat adenosine en hyaluronidase evenveel glycocalyxvolume toegankelijk maken voor bloed. De toename in bloedvolume door de afbraak van glycocalyx met behulp van hyaluronidase ging niet gepaard met een toename in bloedstroom (figuur 3). Dit suggereert dat de weerstandsvaten niet open zijn gaan staan. De toename in bloedstroom tijdens adenosine was voor en na hyaluronidase gelijk (figuur 4). Deze resultaten laten zien dat 1) tijdens adenosine het glycocalyxvolume in het hart toegankelijk wordt voor stromend bloed en 2) dat een verandering van de glycocalyx geen effect heeft op de bloedstroom.

\section{Speelt de glycocalyx een rol in zuurstof uitwisseling in het hart?}

De glycocalyx bevindt zich met name in de haarvaten, de bloedvaatjes waar de uitwisseling van zuurstof, voedingsstoffen en afvalstoffen plaatsvindt. De zuurstofuit- 


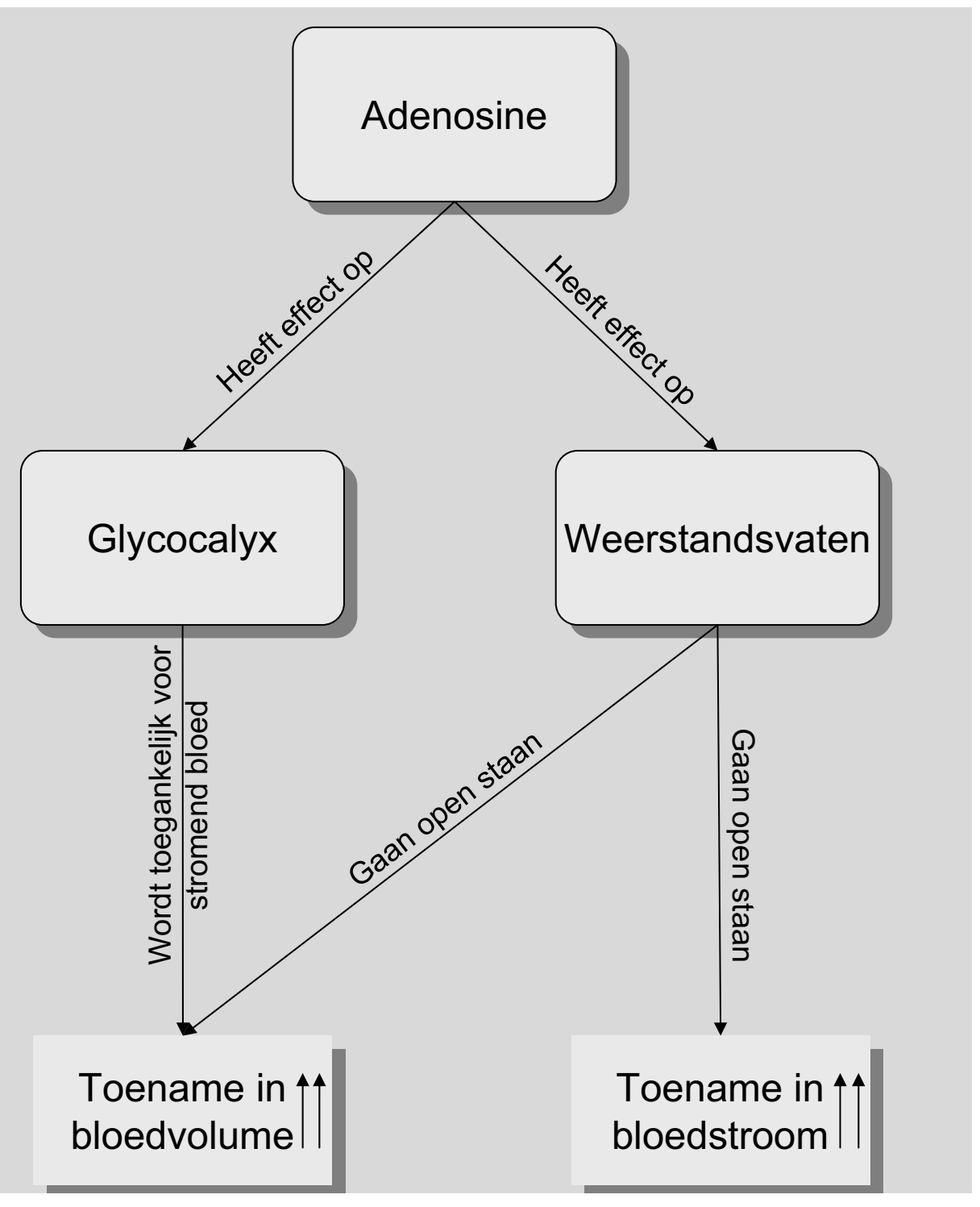

Figuur 2 Het effect van adenosine op de glycocalyx en weerstandsvaten. Met een intacte glycocalyx zal tijdens de toediening van adenosine er een toename in volume en bloedstroom zijn. Het bloedvolume neemt toe doordat de glycocalyx toegankelijk wordt voor bloed en door het open gaan staan van de weerstandsvaten. Dit laaste zorgt er ook voor dat er een toename in bloedstroom is. 


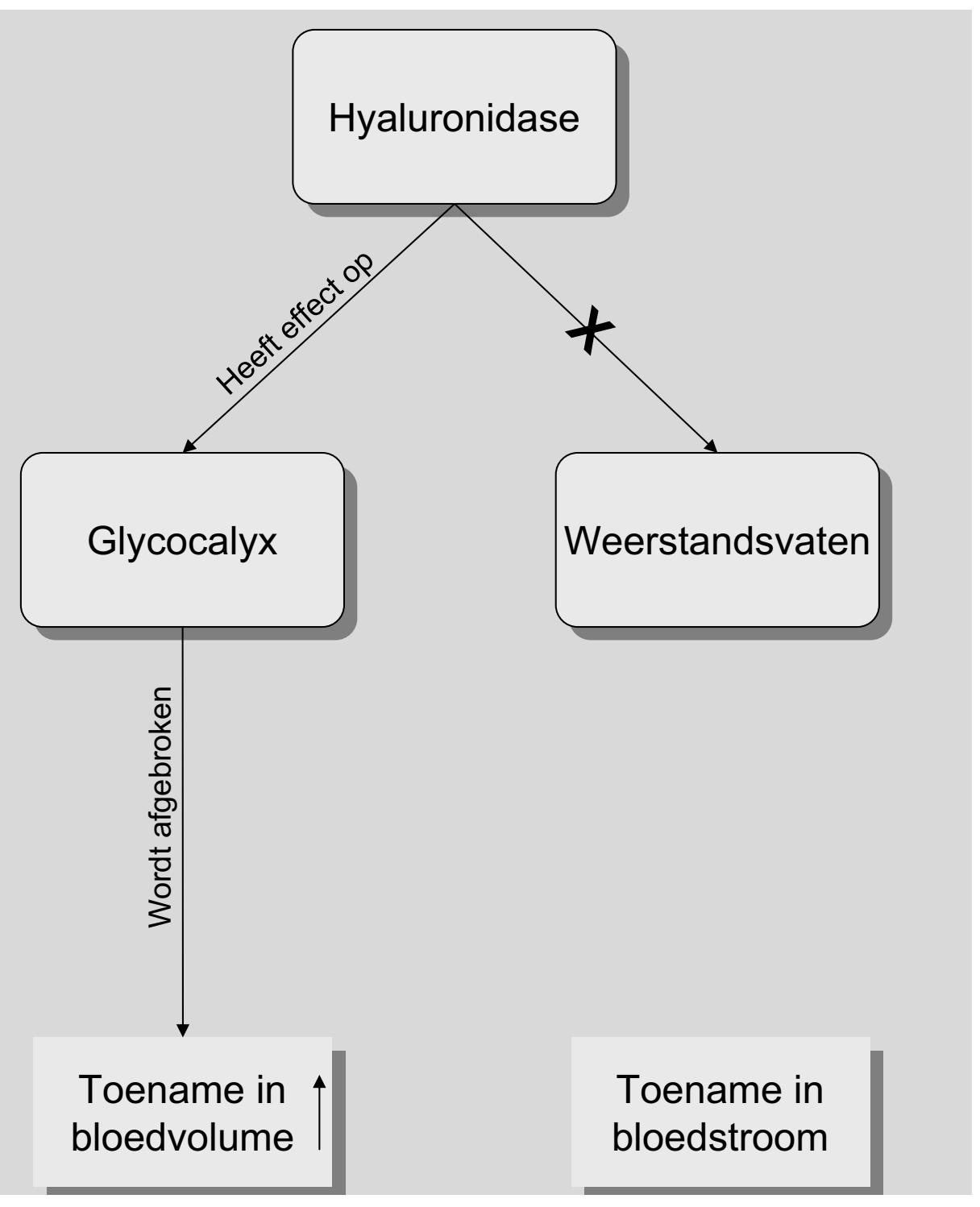

Figuur 3 Het effect van hyaluronidase op de glycocalyx en weerstandsvaten. Na hyaluronidase is de glycocalyx afgebroken en hierdoor is het bloedvolume toegenomen. Hyaluronidase heeft geen effect op de weerstandsvaten waardoor de bloedstroom niet verandert. 


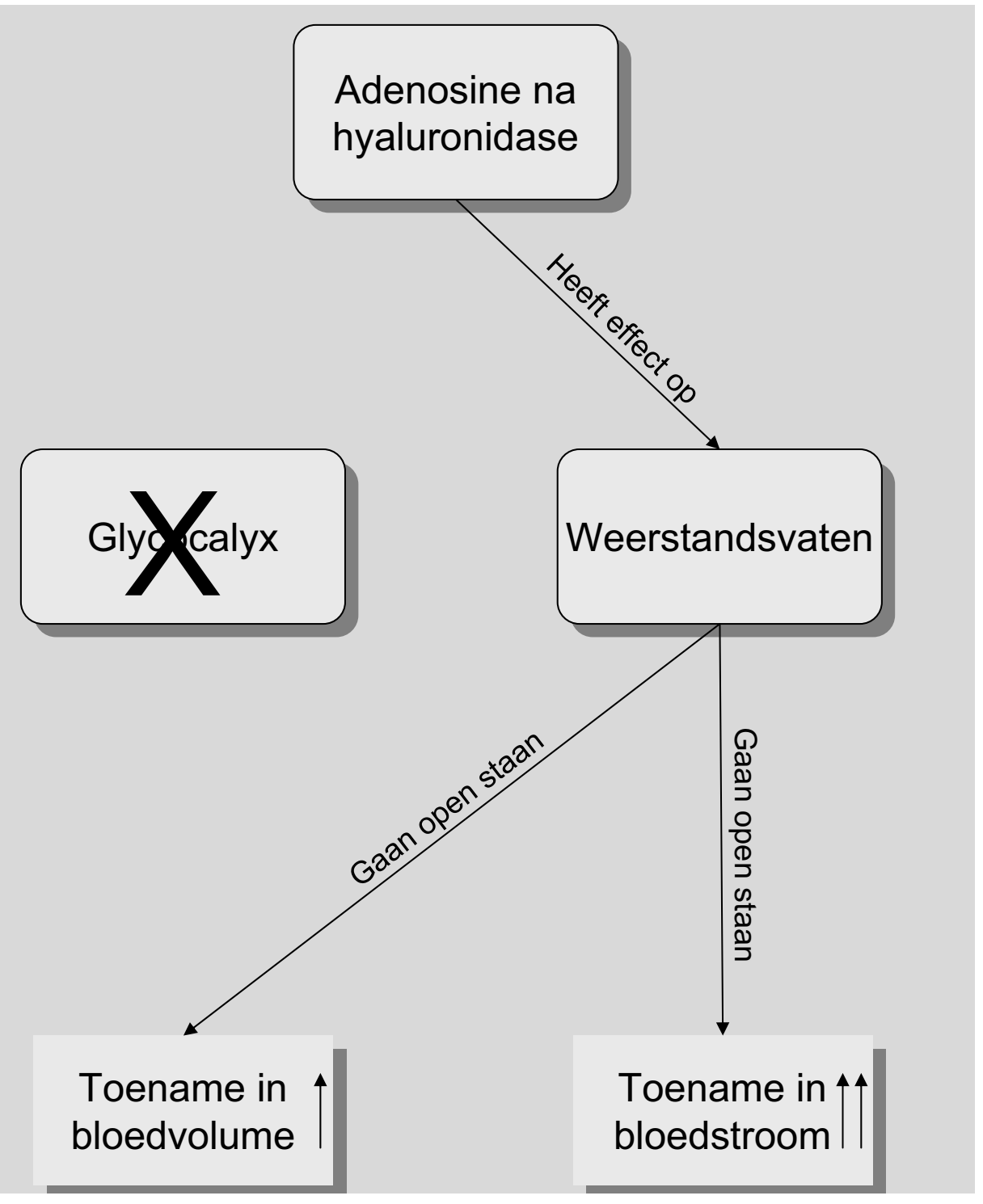

Figuur 4 Het effect van adenosine na hyaluronidase op de glycocalyx en weerstandsvaten. Wanneer adenosine na hyaluronidase wordt gegeven is er geen glycocalyx meer die toegankelijk gemaakt kan worden. Adenosine heeft alleen nog maar een effect op de weerstandsvaten. Door het open gaan staan van de weerstandsvaten zal het bloedvolume en de bloedstroom toenemen. 
wisseling in het hart is gemeten in de aanwezigheid van de glycocalyx alsook na behandeling met hyaluronidase of de toediening van infuusvloeistof (controlemeting). Na verwijdering van de glycocalyx was er een toename in zuurstofextractie terwijl de zuurstofextractie in de controlemetingen gelijk bleef. De aanwezigheid van de glycocalyx lijkt dus de zuurstof uitwisselingscapaciteit te beperken. Het toegankelijk maken van glycocalyxvolume voor bloed kan dus een mechanisme zijn waardoor het hart de zuurstof uitwisselingscapaciteit kan aanpassen aan zijn behoefte.

\section{Interpretatie}

Adenosine verandert de eigenschappen van de glycocalyx. Dit resulteert in een verhoogde toegankelijkheid voor stromend bloed in deze laag. De toename in volume tijdens adenosine bestaat dus niet alleen uit het open gaan staan van weerstandsvaten maar voor een aanzienlijk deel (ongeveer de helft) uit glycocalyx die toegankelijk wordt voor bloed. Dit houdt in dat volumeveranderingen, in tegenstelling tot wat in de tekstboeken staat, ook op niveau van haarvaten plaatsvindt. Het volume kan dus daar waar de uitwisseling van zuurstof en voedingstoffen plaatsvindt gereguleerd worden. Onze resultaten laten verder zien dat de toename in bloedtoegankelijk glycocalyxvolume niet gepaard gaat met veranderingen in bloedstroom. Dus wanneer er schade is opgetreden aan de glycocalyx is deze niet te bepalen aan de hand van een veranderde bloedstroom.

Het toegankelijk maken van de glycocalyx voor bloed had een positief effect op de uitwisselingscapaciteit voor zuurstof van het hart. Hoewel een kortdurende afbraak van glycocalyx gunstig lijkt voor de uitwisseling van zuurstof in het hart, zal langdurige afwezigheid van de glycocalyx waarschijnlijk op de langere termijn nadelige gevolgen hebben voor patiënten. Het hart zal zich vermoedelijk gaan aanpassen aan de nieuwe situatie door het verkleinen van het vaatvolume zodat het bloedvolume ongeveer gelijk wordt aan dat gemeten met een intacte glycocalyx. Bij een verhoogde zuurstofvraag van het hart, bv. tijdens inspanning, zal het hart zich echter minder goed kunnen aanpassen. Er is namelijk een verminderend glycocalyxvolume aanwezig dat toegankelijk gemaakt kan worden voor stromend bloed om hiermee de uitwisseling van zuurstof te bevorderen. Het niet adequaat kunnen aanpassen van aanbod aan vraag door glycocalyxschade, zou dan kunnen bijdragen aan de pijn op de borst 
bij patiënten zonder noemenswaardige vernauwing in een van hun kransslagaders.

\section{Wat nog te doen}

Het mechanisme hoe vaatverwijdende middelen, zoals adenosine, de glycocalyx veranderen is nog onbekend en vereist verder onderzoek. Het enzyme hyaluronidase breekt de glycocalyx grotendeels af door essentiële componenten uit de glycocalyx te verwijderen. Onze data laat zien dat ook tijdens de toediening van adenosine een deel van de glycocalyx afgebroken wordt. Wanneer hyaluronidase na adenosine werd gegeven was er een verdere afbraak van de glycocalyx wat erop duidt dat adenosine de glycocalyx in mindere mate afbreekt dan hyaluronidase. Verder bestaat de mogelijkheid dat er ook een verandering in lading van de glycocalyx plaatsvindt. Dit heeft tot gevolg dat er wellicht niet alleen afbraak plaatsvindt maar ook een verandering in eigenschappen van de glycocalyx.

In het hart is er tijdens adenosine een toename in bloedvolume in de vaten gemeten, terwijl op lichaamsniveau het bloedvolume gelijk bleef en het anatomisch volume afnam. Deze data geven aan dat er niet overal in het lichaam hetzelfde lijkt te gebeuren tijdens systemische toediening van adenosine. In sommige organen zal het bloedvolume toenemen, zoals in het hart, terwijl in andere organen het bloedvolume waarschijnlijk afneemt. Om te bepalen of dit ook daadwerkelijk zo is hebben we bij ratten verschillende organen uitgenomen (zoals hart, long, lever, nier, spieren en hersenen), in de aan- en afwezigheid van adenosine. In de nabije toekomst zullen we in de verschillende organen het aantal met bloed doorstroomde capillairen tellen en deze gaan vergelijken met het totaal aantal capillairen. Door de data van de adenosine groep te vergelijken met de controle groep kunnen we te weten komen of adenosine inderdaad leidt tot uitval van gebruikte capillairen in bepaalde organen en een toename van het aantal capillairen dat gebruikt wordt in andere organen.

\section{Klinische toepassing}

De data uit dit proefschrift suggereren dat glycocalyxschade nadelige gevolgen heeft voor op het elkaar aanpassen van vraag en aanbod van zuurstof in het hart en dat metingen van bloedvolume in de kransvaten van het hart dus zinvol kunnen zijn 
in patiënten die pijn op de borst en geen noemenswaardige stenose hebben in een kransslagader. Een belangrijke stap voordat deze studie naar de kliniek vertaald kan worden, is het ervoor zorgen dat de methode patiëntvriendelijk wordt. Dit laatste zou kunnen door gebruik te maken van beeldvorming, zoals MRI, al dan niet in combinatie van niet schadelijke belletjes die hetzelfde gedrag vertonen als rode bloedcellen. De meetmethode zou dan standaard uitgevoerd kunnen worden bij patiënten met pijn op de borst tijdens inspanning (zoals fietsen of (trap)lopen). Een mogelijke therapie voor deze personen zou het toedienen van glycocalyx componenten kunnen zijn zodat een beschadigde glycocalyx weer hersteld wordt.

\section{Conclusie}

In dit proefschrift is er aangetoond dat toename in bloedvolume tijdens de toediening van vaatverwijdende stoffen voor de helft bestaat uit een toename in toegankelijkheid van de glycocalyx voor stromend bloed. Deze bevinding is in tegenstelling tot dat wat in de tekstboeken staat. Daar wordt er vanuit gegaan dat veranderingen in volume komen door het open gaan staan van weerstandsvaten. Een beschadiging van de glycocalyx wordt niet opgemerkt wanneer er alleen naar bloedstroom wordt gekeken aangezien de doorbloeding van het hart onafhankelijk van de glycocalyx kan veranderen. Schade aan de glycocalyx kan wel worden waargenomen wanneer er naar een bloedvolume verandering wordt gekeken. Aangezien glycocalyxschade in verband wordt gebracht met vaatwand problemen, is het meten van glycocalyxvolume wellicht een belangrijke nieuwe meetmethode om patiënten op te sporen die geen noemswaardige stenose hebben, maar wel een verhoogd risico lopen op hartproblemen. 



\section{Dankwoord}


Na vijf jaar en 2 universiteiten is het moment dan daar; mijn promotie-traject zit er bijna op, mijn proefschrift is af. Promoveren doe je nooit alleen, iemand geeft je de kans om onderzoek te doen en je krijgt begeleiding bij het verzamelen en opschrijven van data wat resulteert in artikelen en uiteindelijk, het proefschrift. Ik wil bij deze dan ook een aantal mensen bedanken die hebben bijgedragen aan de totstandkoming van dit proefschrift. Ik hoop dat ik niemand te kort doe in dit dankwoord en als wel, dan bied ik op voorhand mijn excuses aan.

Allereerst Jurgen van Teeffelen; mijn begeleider en co-promotor. Net nadat ik de kans had gekregen om me bezig te gaan houden met het glycocalyx onderzoek heb je gezegd dat de deur altijd open zou staan, vaak heb ik me afgevraagd of je dit ook had gezegd als je had geweten hoe vaak ik naast je bureau zou staan. De ene keer om een vraag te stellen, soms om je op de hoogte te brengen van de laatste resultaten en andere keren om ideeën en inzichten uit te wisselen. Ik heb veel van je kunnen leren en ben je hier erg dankbaar voor. Ik moest veel leren over onderzoek doen, jij begon aan een nieuwe uitdaging door een onderzoeker te gaan begeleiden maar samen hebben we het voor elkaar gekregen, en ik ben er trots op. Je bent altijd enthousiast geweest, was er altijd met advies en hebt me geholpen daar waar ik wel wat hulp kon gebruiken. Ik verheug me erop om ook in de toekomst nog af en toe samen te werken.

Hans Vink; mijn promotor. Dank je voor de kans die je me hebt gegeven om deel uit te maken van de glycocalyx groep. Het was een eer om opgeleid te worden door de enige glycocalyx-expert die er is. Wanneer ik data had waar ik nog niks van kon maken was het na een gesprek met jou vaak ineens duidelijk. Ook wanneer ik het echt even niet meer zag zitten, en me afvroeg hoe dit ooit tot een goed einde gebracht kon worden, wist je precies wat je moest zeggen. Na twee jaar had je een verassing, je besloot naar Maastricht te verhuizen....met de hele groep. Enigszins met knikkende knieën heb ik mijn spullen gepakt en ben ik verhuisd. Een hele ervaring rijker en met een rugzak vol met kennis is het nu tijd voor de volgende uitdaging. Ook daar heb jij je steentje aan bijgedragen. Dank je wel voor het in mij (blijven) geloven.

Jos Spaan; mijn andere promotor. De laatste van de drie personen die gezamenlijk hebben besloten dat ik deel mocht gaan uitmaken van Medische Fysica. Helaas heeft onze samenwerking niet zolang geduurd als vooraf gedacht en ik ben blij dat je alsnog 
mijn promotor wilde zijn. De laatste jaren konden we niet meer zo vaak over data discussiëren maar probeerde nog wel even snel de laatste ontwikkelingen te bespreken tijdens congressen. Dank je wel voor voor je adviezen en de kans die je me hebt gegeven.

Mark Post; halverwege mijn promotie veranderde ik van universiteit en kwam ik bij de afdeling Fysiologie. Hier ben ik verder gegaan met mijn onderzoek en dat was zonder jou nooit gelukt, bedankt.

Heleen en Geertje; mijn paranimfen en zoveel meer dan dat. Tijdens mijn promotie kon ik altijd bij jullie terecht. Uren hebben we zitten praten over de meest uiteenlopende onderwerpen, hebben we ons hart bij elkaar kunnen luchten, hebben we samen gehuild en nog veel meer gelachen. Inmiddels gaan we alle drie onze eigen weg, in totaal verschillende richtingen en ik wens jullie alle succes en geluk van de wereld toe. Dank je wel voor jullie steun en vriendschap, ik hoop dat die nog lang mag duren.

Mijn kamergenoten; in het bijzonder Adrian, Vincenza en Bernard. Maar ook Sanne, Bram en Pieter. Adrian, twee jaar lang heb ik met veel plezier een kamer met je gedeeld en ik geloof niet dat ik je ooit niet heb zien lachen. Je bent zonder twijfel de meest attente persoon die ik ooit heb leren kennen. Mulţumesc. Bernard, eerst waren er alleen de verhalen en de beroemde foto's van de glycocalyx, waarvan er nu een op de omslag van mijn proefschrift staat. De afgelopen drie jaar heb ik het genoegen gehad je ook te leren kennen. Uren lang hebben we geprobeerd de mysteries van de glycocalyx te ontrafelen en statistiek de baas te worden. Dank je wel voor je hulp en gezelschap. Vincenza, your were a great roommate and a nice friend. Because of your great sense of humour and Mediterranean temperament there was never a dull moment at work. Thank you! I wish you all the luck with your PhD project and can't wait to get your thesis.

De nog niet genoemde leden van de glycocalyx groep; Mirella, Nadja, Sjak, Sjef, Bart, Dan, Hanneke, Martijn, Max, Hans, Marijn en Alina. De discussies op de afdeling, de gesprekken op het lab, het plezier tijdens de congressen. Dank je wel.

Carin; uren hebben we samen zitten pipetteren, facsen en hydrolyseren. Tijdens het werk was het altijd gezellig en wat hebben we vals meegezongen met de radio. Zelfs 
na mijn vertrek naar Limburg bleef je langer op die dagen dat ik even terug was om mee bergen werk te verzetten. Je zat altijd vol verassingen en ik ben je dankbaar voor alles wat je voor me hebt gedaan. Cor, Elly, Dirk, Anneke, Marion, Aniek en Arne. De experimenten hadden nooit plaats kunnen vinden zonder jullie hulp. Dank jullie wel voor jullie kennis, ondersteuning en gezelligheid op de OK.

Naast werk was er ook tijd voor gezelligheid, vaak gewoon op de afdeling. Met veel plezier heb ik de afgelopen jaren de filmavonden georganiseerd. Ik wil iedereen die kwam bedanken voor de gezellige avonden. Onder het genot van pizza, bier of wat fris hebben we veel films gezien, sommige waren echt goed, andere iets minder, maar gezellig was het altijd.

Ik ben mijn promotie begonnen bij Medische Fysica in het AMC waar ik twee jaar met veel plezier heb gewerkt. De laatste paar jaar heb ik met net zoveel genoegen in Maastricht gewerkt bij Fysiologie. Ik zou iedereen van deze afdelingen stuk voor stuk persoonlijk willen bedanken, maar het zijn er zoveel en ik zou het mezelf nooit vergeven als ik iemand zou vergeten dus bij deze wil ik jullie allemaal bedanken voor alle leuke momenten. The last year I have been working at the Center for Ultrasound, Molecular Imaging and Therapeutics in Pittsburgh and I really enjoy working there. Thank you for accepting me as part of the group.

Mensen die ik ook niet wil vergeten zijn mijn vrienden. Silvy, Yvonne, Sjoerd, Lisette, Rolf, Karlien en Ilse. Al jaren zijn we bevriend en ik hoop dat dit voor altijd zo blijft. Dankzij jullie was er veel meer dan werk alleen.

Als laatste wil ik mijn familie bedanken voor hun steun door dik en dun. Papa, mama en Stephan, jullie zijn er altijd voor mij geweest en dat betekent alles voor mij. Dank je wel voor alles wat jullie voor mij hebben gedaan.

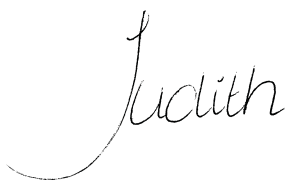


Curriculum Vitae 
Judith Brands was born on December 9, 1979 in Oss, the Netherlands. From 1992 until 1998 she attended secondary education (VWO) at the Maasland College in Oss. From 1998 to 2004 she studied Biomedical Engineering at the Eindhoven University of Technology. During the master phase she performed an internship on the implementation of hemodynamic boundary conditions in a model of the heart and an international internship at the Department of Mechanical and Manufacturing Engineering, Trinity College Dublin, Ireland, where she studied the relationship between stress level and tissue damage of coronary arteries. Her graduation project at the division Biomechanics and Tissue Engineering was on characterisation of abdominal aortic aneurysms using a non-invasive method. Next, she started as a PhD-student at the department of Medical Physics at the University of Amsterdam - Academic Medical Center. In 2006 she continued her research at the department of Physiology at Maastricht University. During this time, she studied agonist-induced modulation of the glycocalyx in the systemic and coronary circulation. The most important findings of her research are described in this thesis. As of November 2009 she is working as a Post-Doc at the Center for Ultrasound Molecular Imaging and Therapeutics at University of Pittsburgh Medical Center (UPMC). 